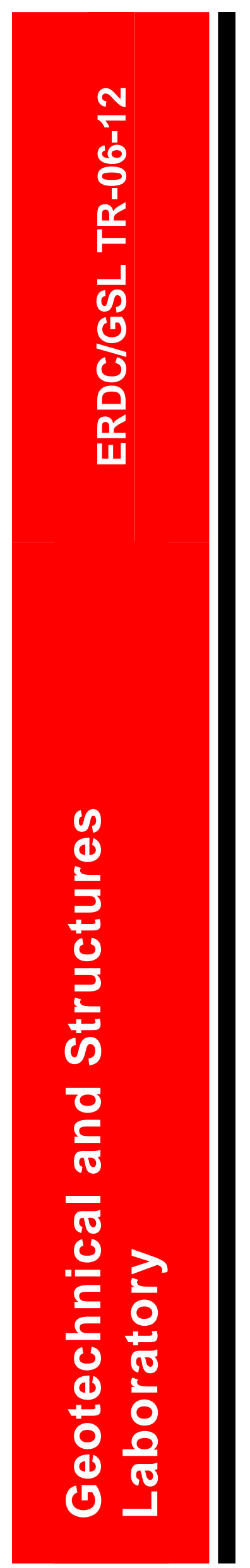

Battlespace Gap Definition and Defeat

\title{
Expedient Gap Definition Using 3D LADAR
}

Lulu Edwards and Sarah R. Jersey

September 2006

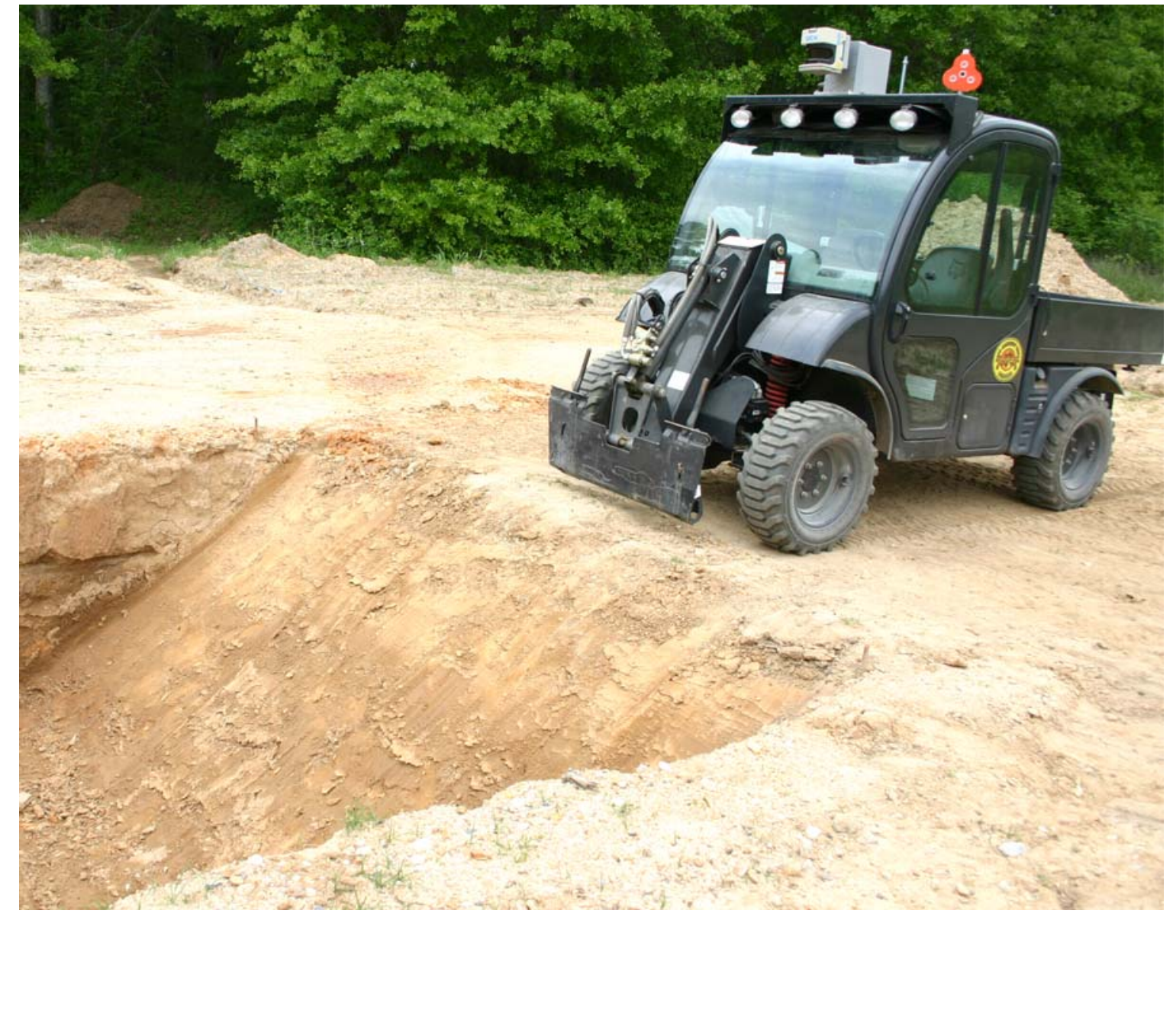

Approved for public release; distribution is unlimited. 


\section{Expedient Gap Definition Using 3D LADAR}

Lulu Edwards and Sarah R. Jersey

Geotechnical and Structures Laboratory

U.S. Army Engineer Research and Development Center

3909 Halls Ferry Road

Vicksburg, MS 39180-6199

Final report

Approved for public release; distribution is unlimited 
ABSTRACT: This report presents a summary of the testing and analyses performed to determine the capabilities of a 3D LADAR (laser detection and ranging) system developed by Autonomous Solutions, Inc. (ASI). Under the direction of the U.S. Army Engineer Research and Development Center (ERDC), ASI has developed an algorithm to reduce the 3D point cloud acquired with the LADAR system into sets of 2D profiles that describe the terrain. This report provides data for (1) evaluating the ranging sensors that could potentially be used for measuring the geometry of a terrain gap, (2) reviewing results of the validation testing of the LADAR system, and (3) developing guidance for using LADAR systems to characterize terrain gaps. Validating the 3D LADAR system included the following tasks: (1) determining the effect of slope and depth of a gap on the measurements acquired with the LADAR system, (2) determining the effect of ambient light conditions on the measurements taken with the LADAR system, (3) determining the effect of different surface materials/textures on the measurements taken with the LADAR system, and (4) determining the effect of fog and dust on the measurements taken with the LADAR system. The project described in this report is part of the Battlespace Gap Definition and Defeat Army Technology Objective program.

DISCLAIMER: The contents of this report are not to be used for advertising, publication, or promotional purposes. Citation of trade names does not constitute an official endorsement or approval of the use of such commercial products. All product names and trademarks cited are the property of their respective owners. The findings of this report are not to be construed as an official Department of the Army position unless so designated by other authorized documents.

DESTROY THIS REPORT WHEN NO LONGER NEEDED. DO NOT RETURN IT TO THE ORIGINATOR. 


\section{Contents}

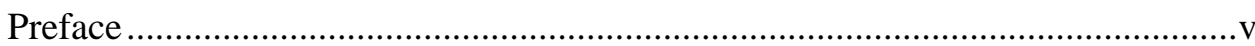

Executive Summary …................................................................................... vi

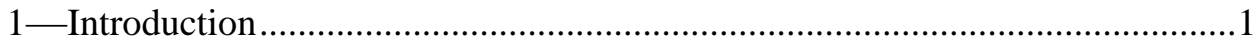

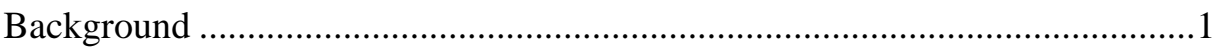

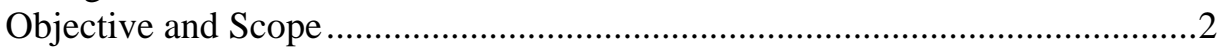

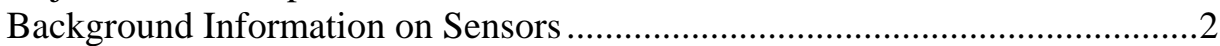

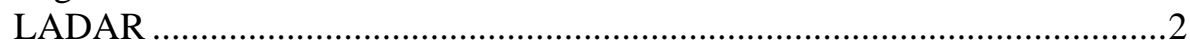

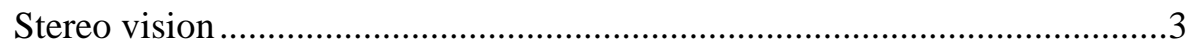

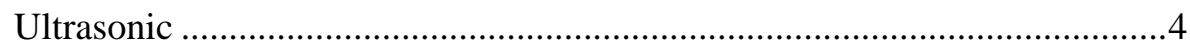

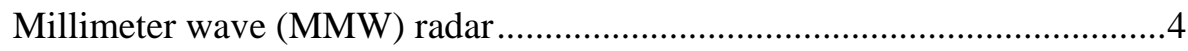

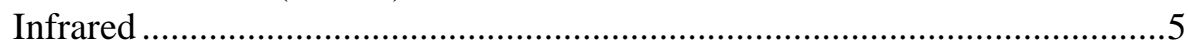

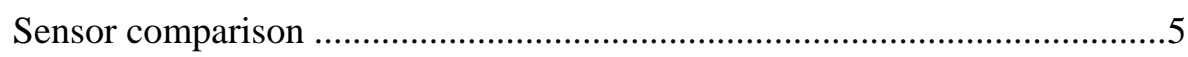

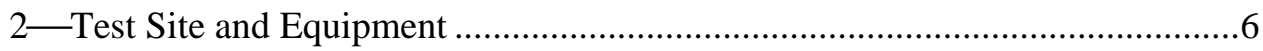

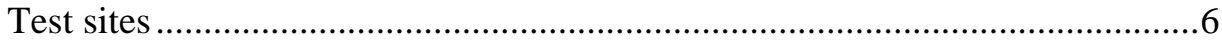

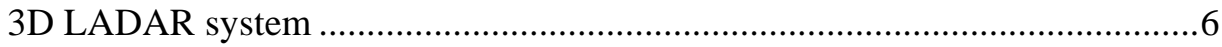

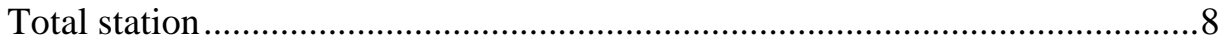

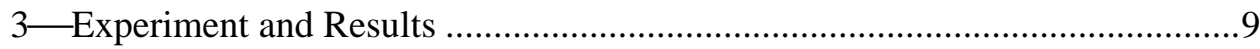

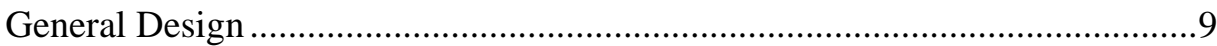

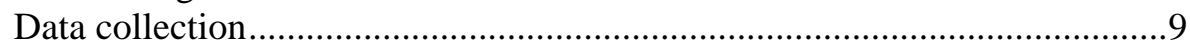

Registration of data sets ....................................................................... 10

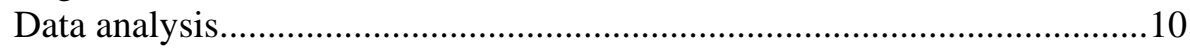

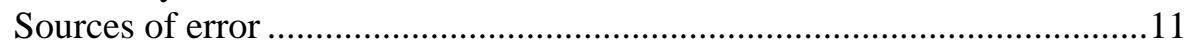

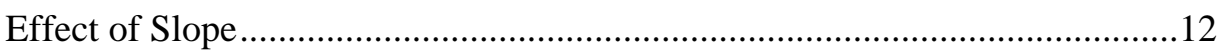

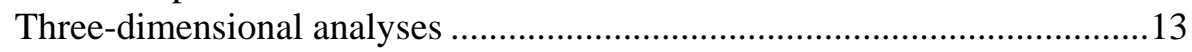

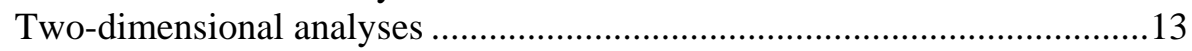

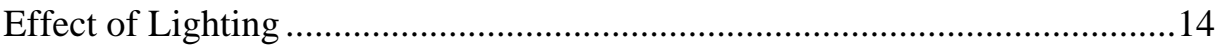

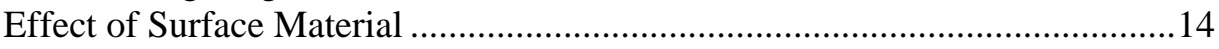

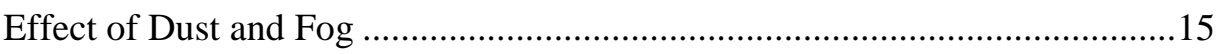

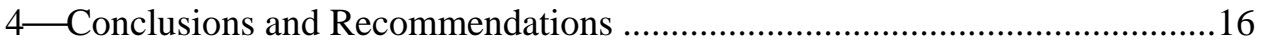

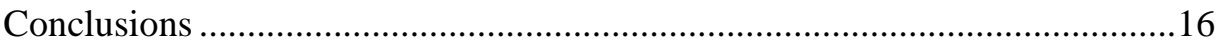

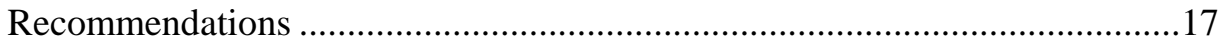

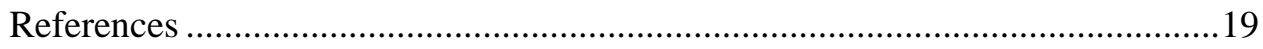


Figures 1-123

Photos 1-21

Appendix A: Summary of Results...............................................................A1

SF 298 


\section{Preface}

This report provides a summary of testing and analyses performed with a commercial-off-the-shelf (COTS) three-dimensional laser detection and ranging (LADAR) system for rapid terrain mapping. The research is being conducted to support units charged with route reconnaissance and maneuver planning.

The investigation reported herein is part of the Battlespace Gap Definition and Defeat (GAP) Army Technology Objective (ATO) Program currently sponsored by Headquarters, U.S. Army Corps of Engineers, Washington, DC. Funding for this investigation was provided under the Battlespace Gap Definition and Defeat ATO IV.GC.2004.02.

The GAP Program is conducted by the U.S. Army Engineer Research and Development Center (ERDC) in conjunction with the U.S. Army TankAutomotive Research, Development, and Engineering Center. Personnel of the ERDC Geotechnical and Structures Laboratory (GSL), Vicksburg, MS, prepared this report. The findings and recommendations presented herein are based upon the testing and analyses of a COTS LADAR system developed by Autonomous Solutions, Inc., at ERDC, Vicksburg, MS. The required field testing was conducted from January to June 2005.

The research team consisted of Lulu Edwards, Sarah Jersey, and Timothy McCaffrey, Airfields and Pavements Branch (APB), GSL. Edwards and Jersey prepared this publication under the supervision of Don R. Alexander, Chief, APB; Jeb Tingle, GAP Program Manager; Dr. Albert J. Bush III, Chief, Engineering Systems and Materials Division; Dr. William P. Grogan, Deputy Director, GSL; and Dr. David W. Pittman, Director, GSL.

COL Richard B. Jenkins was the Commander and Executive Director of ERDC. Dr. James R. Houston was Director.

Recommended changes for improving this publication in content and/or format should be submitted on DA Form 2028 (Recommended Changes to Publications and Blank Forms) and forwarded to Headquarters, U.S. Army Corps of Engineers, ATTN: CECW-EWS, Kingman Bldg, Room 321, 7701 Telegraph Road, Alexandria, VA 22315. 


\section{Executive Summary}

Personnel of the U.S. Army Engineer Research and Development Center (ERDC), Vicksburg, MS, conducted field testing of a commercial-off-the-shelf (COTS) three-dimensional laser detection and ranging (LADAR) system at ERDC, Vicksburg, MS, from January to June 2005. The LADAR system, developed by Autonomous Solutions, Inc. (ASI), was tested for accuracy in measuring the geometry of terrain gaps. The influence of slopes, surface materials, light, fog, and dust were investigated.

The major tasks performed included the following:

a. Evaluation of ranging sensors that could potentially be used for measuring the geometry of a terrain gap.

$b$. Verification of the capabilities of the COTS LADAR system.

c. Development of guidance for using LADAR systems to characterize terrain gaps.

The following conclusions were derived from the experiments and subsequent analyses:

a. The LADAR system is a much more efficient means of defining gap geometry than conventional survey techniques. With the LADAR system, approximately 50,000 points were reported in $1 \mathrm{~min}$, versus 220 points in a span of 30 min with a traditional total station.

$b$. Both three- and two-dimensional analyses indicated that the LADAR system is effective for gaps with approach and departure slopes up to $40 \mathrm{deg}$ at a standoff distance of $0 \mathrm{~m}$. Increasing the gap approach slope to $45 \mathrm{deg}$, on average, approximately triples the error of the LADAR system. At 90-deg approach slopes, the LADAR system is no longer effective.

c. Increasing the standoff distance from $0 \mathrm{~m}$ to $1.5 \mathrm{~m}$ limits the LADAR field of view and the error in the measurements.

d. Ambient light conditions did not significantly affect the LADAR measurements. At peak solar radiation, 1100 and $1300 \mathrm{hr}$, the errors were only slightly higher than the average (approximately 12 percent). 
Overall, the measurements were consistent for the duration of the test, from 0600 to $2100 \mathrm{hr}$.

e. LADAR profiles for sand, soil, riprap, grass, gravel, and mud were similar to the total station profiles, indicating that the LADAR system can provide reasonably accurate measurements for the six surface materials evaluated.

$f$. The accuracy of the LADAR system was adversely affected by particles suspended in air, such as fog or dust. The LADAR could not penetrate the particles in the air, and thus returned false terrain measurements.

g. A total station was used as the ground truth in this investigation. However, there are several possible sources of error with this device, including allowing the prism rod to sink into the surface and not holding the rod plumb.

h. A systematic error, due to a calibration problem, was discovered in all data sets, resulting in an increased error of $2 \mathrm{~cm}$ from the driver side profile to the center profile, and from the center profile to the passenger profile.

Results of this experiment were used to evaluate ASI's LADAR system for defining the geometry of a terrain gap under a variety of conditions. The results from this experiment indicate that profiles measured with the LADAR system have slopes similar to the profiles measured with a total station. Although the error of the system varies, the LADAR system is effective in different lighting conditions, on different surface materials, and on different gap slopes. This system is much more efficient and safe in a battlefield environment because gap geometry data can be rapidly collected from a manned or unmanned vehicle, as opposed to physically surveying gaps with traditional survey equipment. Additional details concerning the LADAR equipment and results from the experiment are contained in Chapters 2 and 3 of this report. Conclusions and recommendations are presented in Chapter 4. 


\section{Introduction}

\section{Background}

The Battlespace Gap Definition and Defeat (GAP) Program is conducted by the U.S. Army Engineer Research and Development Center (ERDC) in conjunction with the U.S. Army Tank-Automotive Research, Development, and Engineering Center. The purpose of this program is to develop technologies that will assist future combat systems in maneuvering vehicles in unknown terrains by facilitating the ability to navigate natural or manmade gaps 1.5 to $4.0 \mathrm{~m}$ wide. Before vehicles can transit such gaps, these technologies must identify and define these gaps under all environmental conditions such as rain, fog, dust, and varying lighting conditions. Adequate characterization of terrain gaps includes definition of key soil properties and gap geometry.

Acquiring high-fidelity information about unknown terrains can speed up a mission by equipping military forces with the capability to make informed choices. Terrain gaps pose a potentially threatening and time-consuming problem for vehicles traveling in unknown terrain. The ability to rapidly map the terrain of a potential gap crossing site and utilize this information to perform vehicle mobility assessments in near real-time will greatly enhance route reconnaissance capabilities in the theater of operations and will result in faster, safer troop movements. Terrain gaps are defined as natural or man-made openings in the ground that could prevent a vehicle from crossing. Knowledge of the gap profile is necessary to make informed choices about avoiding or defeating the gap. If the length is short enough, the vehicle can detour around the gap. If not, the gap must be crossed. The profile of the gap can be used to define the width, depth, and slope, and these properties can be used to determine the feasibility of crossing the gap. Quickly making decisions about defeating a gap requires a sensor that can evaluate the terrain and software that can extract the profile of the gap.

Autonomous Solutions, Inc. (ASI), has developed a three-dimensional (3D) laser detection and ranging (LADAR) sensor package that uses a commercial-offthe-shelf 2D LADAR sensor (SICK LMS 291). This LADAR sensor defines the geometry of the gap by creating a set of $2 \mathrm{D}$ profiles of the gap. The LADAR sensor has a hemispherical field of view such that it can measure the range to surfaces with millimeter to centimeter resolution depending on the distance from the sensor. This range information is represented as a 3D point cloud, and ASI has developed an algorithm to extract from this 3D point cloud any user-defined number of 2D slices. ASI has incorporated this sensor and algorithm into their 
custom software, Mobius ${ }^{\circledR}$, to streamline the profiling process. The entire package is currently mounted on the RAVEN (Rapid Assessment Vehicle Engineer), which is being co-developed by the ERDC for route reconnaissance and rapid airfield assessment (Photo 1).

\section{Objective and Scope}

The primary objective of this investigation was to validate the 3D LADAR system for terrain gap geometry characterization. This objective was accomplished by

a. Determining the effect of slope and depth of a gap on the measurements acquired with the LADAR system.

$b$. Determining the effect of ambient light conditions on the measurements taken with the LADAR system.

c. Determining the effect of different surface materials/textures on the measurements taken with the LADAR system.

d. Determining the effect of fog and dust on the measurements taken with the LADAR system.

The purpose of this report is to provide an evaluation of the capabilities and limitations of the LADAR system under various field conditions.

\section{Background Information on Sensors}

Several sensor technologies can be used for mapping a terrain, or more specifically, determining the geometry of a gap. These methods all have their unique advantages and disadvantages, which should be carefully considered when selecting a sensor. The optimal sensor should be able to work in all lighting conditions and in harsh weather conditions such as fog, rain, and dust. The sensor should be compact enough to mount onto a reconnaissance vehicle and should be capable of rapid data acquisition and processing. The sensor should also have a minimum range capability of at least $10 \mathrm{~m}$ to easily measure a 4-m-wide gap.

\section{LADAR}

LADAR is an acronym for "laser detection and ranging" or "laser radar." LADAR technology has existed since the 1960s, but it has only recently, within the last decade, become more common in research and development. The technology offers a variety of applications that include 3D modeling, automation, urban planning, mapping, surveying, autonomous vehicle navigation, quality control in manufacturing, global climate monitoring, bathymetry, and homeland security issues such as face recognition and surveillance (Cheok and Stone 2004). 
LADAR is an active ranging method in that a laser pulse is transmitted and the return of the laser pulse is used to determine the distance to an object. There are two major categories of LADAR: pulsed time of flight (TOF) and continuous wave $(\mathrm{CW})$. The pulsed TOF method operates by transmitting a laser pulse and measuring the time for the laser pulse to return from a target to calculate the range. CW LADAR calculates range information by indirectly measuring the time of flight, and can be further divided into amplitude modulated CW (AMCW) and frequency modulated CW (FMCW). AMCW LADAR calculates the range to a target by measuring the phase change of a sinusoidal-modulated laser beam (García and Lamela 2001). FMCW LADAR calculates the range to a target by measuring the shift in frequency of a frequency-modulated laser.

These three types of sensors are quite different and, therefore, are usually applied in different ways. TOF LADAR sensors are well suited for long-range purposes in an outdoor environment, but typically have a slower acquisition rate than the other LADAR methods. AMCW LADAR sensors usually have faster acquisition rates compared with the other two types, but are limited in the range (typically less than $50 \mathrm{~m}$ ) and are susceptible to outdoor lighting conditions. FMCW LADAR sensors have more accuracy than the other two sensor types, but are typically not used for rugged, outdoor purposes because of their complicated design (Hebert 2000).

There are several advantages to using a LADAR sensor for terrain mapping. LADAR sensors are usually compact, and they can provide fast, high-resolution, $3 \mathrm{D}$ range information. The ranges of LADAR systems vary, but several sensors are capable of measuring up to $100 \mathrm{~m}$. Because of their increasing demand, LADAR systems are becoming more affordable. Changing ambient light does not hinder LADAR sensors because they are active sensors, meaning that they provide their own illumination. However, LADAR sensors do have limitations. LADAR measurements are adversely affected by harsh environmental conditions such as snow, haze, dust, and rain (Grantham and Meidunas 1998). To compensate for air particles, such as fog and rain, TOF LADAR manufacturers have begun offering a "last pulse measuring" technique. This technique works by using only the last echo detected within a single pulse. This idea is that all echoes prior to the last represent particles in the air, and the last echo represents the target object (Hebert 2000).

\section{Stereo vision}

Stereo vision is a passive sensor technology that uses images from two cameras to determine 3D range information. The range or distance can be calculated by finding matching pixels, i.e., pixels in the two images that represent the same point in space. These matching pixels are usually found by using correlation values or sum of square differences on the two images. The range information can be calculated using a triangulation method, provided that the disparity of matching pixels in the two images and the locations of the two cameras relative to each other are known (Hebert 2000). 
There are several disadvantages to using stereo vision for the purposes of ranging for terrain mapping. The optimal baseline, or the distance between the two cameras, is difficult to determine. The desired measurement range and resolution are both dependent on the baseline. Larger baselines yield higher resolution, but cause matching pixels to be more difficult and susceptible to error. Proper calibration is required to align the images from the two cameras, thus making stereo vision a challenge in a rugged environment (Fernández et al. 1999, Hebert 2000). Because stereo vision is a passive sensor technology, it is extremely dependent on adequate ambient lighting. Also, environmental conditions that decrease visibility (such as fog, rain, and dust) adversely affect the quality stereo vision information (Wijesoma et al. 2004).

Stereo vision technology is constantly improving and changing. Multiple cameras are being used to increase the resolution and solve the optimal baseline problem. Stereo vision is extremely computationally intensive, and until recently, with the improvement in processing power, this has severely limited its effectiveness. Stereo vision systems are also becoming increasingly more compact, making them more useful for robotic applications (Hebert 2000).

\section{Ultrasonic}

Ultrasonic sensors usually work on the TOF principle, where an ultrasonic pulse is transmitted, and the time for this pulse to reach an object and return is used to calculate the range. Ultrasonic sensors are often used in robotics for obstacle detection and ranging because of their low cost, compact size, and simplicity. However, there are many limitations to using ultrasonic sensors for the purpose of determining range, because of their slow response time, low resolution, and large measurement error due to crosstalk, specular reflections, or ambient noise (Fernández et al. 1999). Because ultrasonic sensors are based on the TOF principle and the speed of sound is $340 \mathrm{~m} / \mathrm{sec}$, the response time for ultrasonic sensors is too slow to be used for long ranges (Benet et al. 2002). Ultrasonic sensors are also most accurate when the target surface is perpendicular to the sensor direction, thus limiting the effectiveness of using ultrasonic sensors for terrain mapping (Borenstein and Koren 1988).

\section{Millimeter wave (MMW) radar}

Millimeter wave (MMW) radar is a technology that is used for ranging purposes because of its robustness. MMW radar antennas transmit electromagnetic radiation that is reflected, refracted, and absorbed by objects along the radiation pathway until there is no more radiation left to detect by the sensor. The range of the target object is calculated by comparing the emitted radiation to the object with the radiation that returns to the sensor. Because the radiation can penetrate objects, the range information of multiple objects can be measured.

The frequency band that corresponds to MMW radar is from 30 to $300 \mathrm{GHz}$. The millimeter wavelength range is crucial to the success of the MMW sensor 
technology. This wavelength is smaller than that of conventional microwave radars, which results in better resolution, but large enough to penetrate snow, fog, dust, and rain. In addition to the insensitivity of MMW radar sensors to air particles, MMW range measurements are not affected by ambient lighting. However, the disadvantage of using MMW sensors is that they have low resolution compared with other ranging sensors and large footprints. In addition, to achieve high resolution, larger antenna sizes are necessary (Foessel-Bunting et al. 2001).

\section{Infrared}

Infrared sensors are inexpensive and easily accessible; however, using them for ranging purposes can be complicated because of their nonlinear responses and their dependence on the surface reflectivity of the target object. The range capability of infrared sensors is usually short, from 0 to $5 \mathrm{~m}$. Therefore, these sensors are normally used for detecting and avoiding obstacles (Benet et al. 2002).

To use infrared sensors as dedicated ranging sensors, the behavior of the surface of a target object as it reacts to infrared energy and the angle of the target object surface relative to the infrared sensor must be determined prior to calculating the range. However, determining range is complicated by the fact that the distance to the object must be known prior to characterizing surface reflectivity. Barshan and Aytaç (2003) proposed scanning a target over a specified angular range with an infrared sensor and using the relationship of the intensity and angle to identify the surface. Novotny and Ferrier (1999) proposed combining a sonar sensor with an infrared sensor to more accurately determine the range.

\section{Sensor comparison}

The previous sections provided an overview of potential sensors available for terrain mapping. Each sensor has its advantages and disadvantages, and all of these factors must be considered when choosing a sensor for ranging. When compared with other sensor technologies, LADAR was found to meet many of the requirements needed for gap geometry characterization. Stereo vision requires two or more cameras and precise calibration, but LADAR sensors are compact and have only one component. Ultrasonic sensors, which are based on the speed of sound, are slow compared with LADAR, which is based on the speed of light. Infrared ranging technology has not advanced enough to be a reliable ranging technology for ranges greater than $5 \mathrm{~m}$, while many LADAR sensors can determine range up to $100 \mathrm{~m}$. MMW radar is far superior to the other sensors when it comes to collecting data in fog, rain, or dust. However, MMW radar requires large antennas to acquire high-resolution data, while LADAR systems are capable of collecting high-resolution data with relatively compact equipment. 


\section{Test Site and Equipment}

\section{Test Sites}

Two sites were constructed at the ERDC, Vicksburg, MS, for testing of the LADAR system in different field conditions. One site was used primarily to determine the effect of gap slope and depth on the LADAR sensor, while the second site was used to determine the effect of fog, dust, and surface material/texture on the ability of the LADAR system to produce accurate ranging information.

\section{D LADAR System}

The 3D LADAR system is based on the SICK LMS 291 LADAR scanner. The reported capabilities of this scanner are summarized in Table 1. The SICK LMS 291 operates on the TOF principle, which means that the range of an object is determined by using the time required for the laser beam to travel from the sensor to the object and back. A rotating mirror is used to direct the laser beam so that a semicircle (180 deg) of data is collected from left to right (SICK AG 2002).

The sensor unit is rotated $360 \mathrm{deg}$ in increments of $0.25,0.5$, or 1.0 deg to essentially allow the 2D scanner to collect data in a third dimension. At each rotation increment, a linear scan of 2D points is acquired. A complete 360-deg rotation of the sensor creates a 3D point cloud of the current scene in the field of view. The sensing area covers 360 deg by 180 deg, creating a hemispherical field of view (Figure 1a). Each point in the point cloud represents the distance to an object that the laser has encountered relative to the center of the laser.

This LADAR system captures one linear scan every $200 \mathrm{msec}$, which results in an equivalent data acquisition rate of 1,800 points per second. The accuracy of the LADAR system is dependent on the desired range, where shorter ranges have higher resolution data and longer ranges result in lower resolution data. The LADAR system is accurate within millimeters for distances up to $8 \mathrm{~m}$, centimeters for distances up to $80 \mathrm{~m}$, and decimeters for distances up to $150 \mathrm{~m}$. 


\begin{tabular}{|c|c|}
\hline \multicolumn{2}{|c|}{$\begin{array}{l}\text { Table } 1 \\
\text { Summary of SICK LMS } 291 \text { Capabilities }\end{array}$} \\
\hline Characteristic & \\
\hline Laser protection class & 1 (eye safe) \\
\hline Range & Maximum $80 \mathrm{~m}$ \\
\hline Wavelength & $905 \mathrm{~nm}$ \\
\hline Angular resolution & $0.25^{\circ} / 0.5^{\circ} / 1.0^{\circ}$ (selectable) \\
\hline Response time & $53 \mathrm{~ms} / 26 \mathrm{~ms} / 13 \mathrm{~ms}$ \\
\hline Measurement resolution & $10 \mathrm{~mm}$ \\
\hline $\begin{array}{l}\text { System error (conditions: good } \\
\text { visibility, } \mathrm{Ta}=23^{\circ} \mathrm{C} \text {, reflectivity } 10 \% \text { - } \\
10,000 \% \text { ) }\end{array}$ & $\begin{array}{l}\text { For ranges 1-4 m, typically } \pm 60 \mathrm{~mm} \\
\text { For ranges } 4-20 \mathrm{~m} \text {, typically } \pm 35 \mathrm{~mm}\end{array}$ \\
\hline $\begin{array}{l}\text { Statistical error or standard deviation } \\
\text { (reflectivity } \geq 10 \% \text { ) }\end{array}$ & For ranges 1-20 m, typically $\pm 10 \mathrm{~mm}$ \\
\hline Data interface & RS 232/RS 422 (configurable) \\
\hline Transfer rate & 9.6/19.2/38.4/500 KBaud \\
\hline Switching outputs, standard variants & $\begin{array}{l}3 \times \text { PNP; typ. } 24 \text { V DC; OUT A, OUT B maximum } \\
250 \text { mA, OUT C maximum } 100 \text { mA }\end{array}$ \\
\hline Supply voltage (scanner-electronics) & $\begin{array}{l}24 \mathrm{~V} \mathrm{DC} \pm 15 \% \text { (maximum } 500 \mathrm{mV} \text { ripple), current } \\
\text { requirements maximum } 1.8 \mathrm{~A} \text { (including output load) }\end{array}$ \\
\hline Power uptake & Approx. 20 W (without upload) \\
\hline Electrical protection class & Safety insulated, protection class 2 \\
\hline Interference resistance & According to IEC 801, Part 2-4; EN 50081-1/50082-2 \\
\hline Ambient temperature & Operating: $0-50{ }^{\circ} \mathrm{C}$, Storage: -30 to $70{ }^{\circ} \mathrm{C}$ \\
\hline Enclosure rating & IP 65 \\
\hline Weight & Approx. $4.5 \mathrm{~kg}$ \\
\hline Dimensions & $\begin{array}{l}185 \times 156 \times 210 \mathrm{~mm} \text {; with cables: } 185 \times 156 \\
\times 265 \mathrm{~mm}\end{array}$ \\
\hline Vibration fatigue limit & $\begin{array}{l}\text { According to IEC } 68 \text { Part } 206 \text {, Table } 2 \mathrm{c} \text {, frequency } \\
\text { range } 10-150 \mathrm{~Hz} \text {, amplitude } 0.35 \mathrm{~mm} \text { or } 5 \mathrm{~g} \text { single } \\
\text { impact IEC } 68 \text { Part 2-27, Table } 2,15 \mathrm{~g} / 11 \mathrm{~ms} \\
\text { permanent vibration IEC } 68 \text { Part } 2-29,10 \mathrm{~g} / 16 \mathrm{~ms} \text {. } \\
\text { Shock absorbers are recommended for heavy vibration } \\
\text { and impact demands (e.g. AGV applications). }\end{array}$ \\
\hline
\end{tabular}

Under ERDC direction, ASI has developed an algorithm to condense the 3D point cloud acquired with the LADAR system into sets of 2D profiles that describe the terrain. Each 2D profile describes the shape of the gap along the width of the gap at a distance from the center of the LADAR sensor (Figure 1b). The algorithm has many user-defined parameters that can be set in Mobius ${ }^{\circledR}$, the software used to control the laser. These parameters can be seen in Figure 2 and are described in Table 2. Within Mobius ${ }^{\circledR}$, the 3D point cloud is displayed in real time, updating the image as data are collected (Figure 3). The software application Jaus Plotter displays the 2D profiles, as shown in Figure 4. If all of the profiles are displayed in 2D mode, they will overlap with each other, making it difficult to see details in individual profiles. However, the software is capable of displaying only select profiles. 
The output is saved as a text file, which consists of the depth and distance along the width for each profile. The latitude and longitude are also reported, provided that a GPS system is enabled.

\begin{tabular}{|l|l||}
\hline \hline \multicolumn{1}{|l||}{$\begin{array}{l}\text { Table } 2 \\
\text { 3D LADAR System's User-Defined Parameters }\end{array}$} \\
\hline \hline Parameter & Description \\
\hline \hline \# Scans & Number of linear scans recorded during one complete rotation \\
\hline Mode & $\begin{array}{l}\text { Field of view (FOV) in front of the laser } \times \text { step angle as the LADAR scans } \\
\text { the FOV. Options: } 180 \times 1.0 \text { deg, } 180 \times 0.5 \text { deg, 180 x } 0.25 \text { deg }\end{array}$ \\
\hline Distance & $\begin{array}{l}\text { Desired maximum range and resolution } \\
\text { Options: } 8 \mathrm{~m}(\mathrm{~mm}), 16 \mathrm{~m}(\mathrm{~mm}), 32 \mathrm{~m}(\mathrm{~mm}), 80 \mathrm{~m}(\mathrm{~cm})\end{array}$ \\
\hline Duty Cycle \% & $\begin{array}{l}\text { Rotation speed as the percentage of the full speed. (max speed } \\
=200 \text { rpm) }\end{array}$ \\
\hline Section Size & Distance between each profile \\
\hline Plot Results & Plot results in Jaus Plotter \\
\hline Sample Range & $\begin{array}{l}\text { Width of data collection (half of this width is to the left of the vehicle, and } \\
\text { half of this width is to the right of the vehicle) }\end{array}$ \\
\hline Ignore Canopy & Report only lowest data points in each profile \\
\hline Number Sections & Number of profiles to collect \\
\hline
\end{tabular}

\section{Total Station}

A Zeiss total station (Elta ${ }^{\circledR}$ 50R) was used to obtain "ground truth" data to validate the LADAR system. The total station consisted of the control unit and a target, which was a prism attached to an adjustable rod. The foot, or bottom of the rod, is detachable. Two types of attachments were available: a pointed foot or a flat foot. For stability reasons, the flat foot was preferred, and was used when possible. However, the pointed foot was required for surveying in gaps with steeper slopes.

The surveys were conducted by a two-person team (instrument man and rod man). Coordinates were measured and recorded in all three dimensions with the control unit. The accuracy of the total station was reported to be $5 \mathrm{~mm}$, and the measuring time for one point was 3 sec (Elta Electronics Industries, Ltd. 1996). 


\section{Experiment and Results}

\section{General Design}

Custom-designed gaps were constructed (Photo 2) to evaluate the effectiveness of LADAR technology in characterizing the geometry of terrain gaps. A range of slopes and surface material types were constructed. Environmental factors such as fog, dust, and amount of ambient light were also considered. Plan and profile views of the gaps are shown in Figures 5 and 6.

The profiles measured with the LADAR system were compared with total station measurements to determine the system accuracy. The two data sets were aligned so that every point in both data sets represented approximately the same location on the terrain. The absolute value of the difference between the two data sets was used to quantify the accuracy of the LADAR system.

\section{Data collection}

For each gap, three sets of LADAR data were acquired consecutively, and data were collected at $0.3-\mathrm{m}$ intervals with the total station. The LADAR data were acquired three times to evaluate the consistency of the measurements. Table 3 shows the Mobius ${ }^{\circledR}$ parameter settings that were used for all of the data sets. Because this was a verification experiment, the acquisition speed was not a limiting factor in data collection. Instead, the highest resolution was desired and, to this end, the duty cycle was set to a low value (3 percent), and the LADAR was run for approximately $1 \mathrm{~min}$ to ensure that all data points were acquired.

Profiles of each gap were measured with the total station (Photos 3 and 4) by taking data every $0.3 \mathrm{~m}$ along the gap, starting at the RAVEN parked adjacent to the gap and proceeding to the opposite side of the gap. A grid of data was collected along multiple profiles spaced at $0.3-\mathrm{m}$ increments along the length of the gap to allow both 2D and 3D analysis. For 2D analysis only, three profiles were measured at the following locations with respect to the RAVEN: the driver side wheel path, the center of the vehicle, and the passenger side wheel path. 


\begin{tabular}{|c|c|}
\hline \multicolumn{2}{|c|}{$\begin{array}{l}\text { Table } 3 \\
\text { 3D LADAR Parameters Used }\end{array}$} \\
\hline Parameter & Description \\
\hline \# Scans & 1080 \\
\hline \begin{tabular}{|l|} 
Mode \\
\end{tabular} & $180 \times 0.25 \mathrm{deg}$ \\
\hline Distance & $32 \mathrm{~m}(\mathrm{~mm})$ \\
\hline Duty Cycle \% & 3 \\
\hline Section Size & 0.01 \\
\hline Plot Results & True \\
\hline Sample Range & 5 \\
\hline Ignore Canopy & True \\
\hline Number Sections & 500 \\
\hline
\end{tabular}

\section{Registration of data sets}

To compare the LADAR and total station data, the two data sets had to be registered, or aligned with one another. Three control points were measured in both data sets and used as a means to register the two data sets. The control points were located on top of the RAVEN and were chosen because it would be easy to attach the total station prism securely to these locations without damaging the RAVEN. Two of the control points were locations of removable antennas where the total station prism could be screwed into place with a bolt. The other control point was the location of a GPS Rover attached to the top of the RAVEN with a bolt, which could also be easily removed to attach the total station prism. These control points are illustrated in Figure 7.

The point at which the LADAR sensor rotates is the origin of the LADAR data set (coordinate point 0,0,0), and its location is shown in Figure 8. The control point coordinates in the LADAR data sets were measured physically, and manually added to the LADAR data. Figure 9 illustrates the locations of the three control points with respect to the center of the LADAR sensor. These points were measured with the total station by removing the rod from the prism and attaching the prism to the chosen locations. To compensate for the removal of the rod, the control point coordinates were later corrected by adding the length of the rod to the height measurement. Autodesk AutoCAD ${ }^{\circledR} 2006$ was used to align the control points measured with the total station with the LADAR control points.

\section{Data analysis}

The first step of data analysis was to reformat the LADAR data into the format required by Terramodel ${ }^{\circledR}$. The format for LADAR output from Mobius ${ }^{\circledR}$ grouped the depth and distance along the gap for each profile. Terramodel ${ }^{\circledR}$ required the data to be in a coordinate format (point number: $\mathrm{x}, \mathrm{y}, \mathrm{z}$ ). A LabView program was written to accomplish this reformatting. 
The total station data were reformatted and registered with the LADAR data in AutoCAD ${ }^{\circledR}$ 2006. The total station data and the LADAR data were both imported into Terramodel ${ }^{\circledR}$ for visualization and analysis purposes. Each data set could be viewed and compared in 3D, and 2D profiles could be extracted from the data sets.

The data sets were analyzed in both 3D and 2D to quantify the accuracy of the LADAR system. For a 3D analysis, Terramodel ${ }^{\circledR}$ was used to compare the volumes measured with LADAR and total station. The total station volume was subtracted from the LADAR volume, creating a "cut volume" for the total LADAR volume less than the total station volume, and a "fill volume" for the total LADAR volume greater than the total station volume. The total volume, which was the sum of the absolute value of the cut and fill volumes, was used to quantify the LADAR error, or difference from the total station data. The results of all 3D analyses can be found in Figures 10-25 and are discussed later in this chapter.

For 2D analysis, the absolute difference between 2D LADAR profiles and the total station data sets was calculated for each data point. The LADAR data set was much larger than the total station data set, so comparing the LADAR and total station data sets required that the LADAR data be condensed so that each total station point had a corresponding LADAR value. Three profiles were used for these calculations: the driver side wheel path, passenger side wheel path, and center profiles. The error of the LADAR system was defined as the absolute difference between the measurement systems.

To more accurately describe the accuracy of the LADAR data in 2D, each set of data was divided into three groups: total gap, approach slope, and departure slope. The absolute difference between the total station and LADAR data was calculated at each total station point, and these values were averaged. These values were calculated for each of the three sets of LADAR data (Laser A, B, and C) collected at a particular gap. Laser A, B, and C values for the three groups were averaged, and this number was used to represent the error. The results of all 2D analyses can be found in Figures 26-120 and are discussed later in this chapter.

\section{Sources of error}

In the following sections, the average error (2D analysis) or average total volume difference (3D analysis) is used to quantify the difference between measurements taken with the total station and LADAR system. It should be noted that, while the total station information was used as the ground truth data, these data were also susceptible to errors. The rod must be held level and on the surface at the time of measurement, but it is difficult to accomplish these tasks perfectly each time, especially for gaps with steep slopes.

In some data sets, incorrect measurements resulted from the RAVEN's hydraulic lift mechanism, which is used for various attachments (see Photo 1). Although this mechanism was not physically located at the gap, it caused a shadow effect on LADAR measurements when the RAVEN was near the edge of the gap. The lift mechanism prevented the LADAR sensor from measuring points 
beyond it, thus causing the shadow effect. These erroneous measurements were not included in the 2D profile measurements to show the accuracy capability of the LADAR system. However, the erroneous measurements remained in the 3D volume calculations to allow the system as a whole to be evaluated.

Approximately 80 times more LADAR data were collected than total station data. Because the LADAR data were so dense, they more accurately described areas where the terrain was rough. The total station data were only collected every $0.3 \mathrm{~m}$ and, therefore, could misrepresent rough areas.

Generally, the total station-measured depths were larger (deeper) than the LADAR-measured depth. This systematic error could be partially the result of rod sinkage. Although care was taken to carefully place the rod, the rod usually sank into the ground. The rod would sink even more if the ground or surface material was soft, as was the case with grass and mud.

For all data sets, there was a tilt in the data, causing the average error in the center profile to be approximately $2 \mathrm{~cm}$ higher than the driver side profile, and the passenger side profile to be approximately $2 \mathrm{~cm}$ higher than the center profile. The tilt is a systematic error due to a misalignment of the LADAR sensor and can be corrected in postprocessing. This problem can be corrected in the future by ASI by calibrating the LADAR sensor position.

\section{Effect of Slope}

Gaps with slopes of approximately 5, 10, 15, 20, 30, 40, 45, and 90 deg were evaluated. The slope angles were measured from the horizontal or ground plane. Four gaps, illustrated in Figure 5, were constructed to evaluate the slopes. Photo 1 was taken during the construction of one of the gaps. Each gap was constructed with a different slope on either side to minimize the number of gaps. The width and length of each gap was approximately $4 \mathrm{~m}$.

LADAR data were collected with the RAVEN reconnaissance vehicle at the edge of each gap (standoff of $0 \mathrm{~m}$ ), as well as at a standoff distance of approximately $1.5 \mathrm{~m}$ from the edge (Photos 4-8). LADAR data were collected from both sides of the gaps so that the effect of the slopes could be examined for both approach and departure angles. Three sets of LADAR data were acquired, and the ground truth data were surveyed in a grid pattern with $0.3-\mathrm{m}$ intervals for each gap. Total station data for the gap profiles were collected once, but the control points were remeasured each time the RAVEN was moved to collect another set of LADAR data.

The time required to collect LADAR data was considerably shorter than that required to collect the total station data. LADAR data collection was accomplished in $1 \mathrm{~min}$ and yielded approximately 50,000 data points. The total station survey at 0.3-m intervals yielded approximately 220 points and required $30 \mathrm{~min}$. Measuring each set of control points with the total station required an additional $15 \mathrm{~min}$ because the locations of these points were at the top of the RAVEN and not easily accessible. 


\section{Three-dimensional analyses}

Results of the 3D volume differences between the LADAR and total station are illustrated in Figures 10-23. A summary of the volume subtraction data is provided in Figures 24-25. Table A1 contains the results for all 3D data analyses. For each slope, the LADAR data were collected three times, and the average of the total volume differences for all three data sets was used to quantify the error of the LADAR system.

For a 0-m standoff distance, the LADAR system was effective for slopes up to $40 \mathrm{deg}$, where the average of the total volume differences was $0.95 \mathrm{~m}^{3}$ (range: 0.6 to $1.35 \mathrm{~m}^{3}$ ). The effectiveness of the LADAR system begins to decrease at $45 \mathrm{deg}$, where the average total volume difference approximately triples to $3.43 \mathrm{~m}^{3}$. At $90 \mathrm{deg}$, the LADAR system is no longer effective, as is apparent from the large total volume difference of $8.83 \mathrm{~m}^{3}$. The significant increase in error for slopes greater than 40 deg is evident in the $3 \mathrm{D}$ volume difference plot shown in Figure 24.

On average, increasing the standoff distance to $1.5 \mathrm{~m}$ approximately doubles the total volume difference between the LADAR and total station data for slopes up to $45 \mathrm{deg}$. For all slopes except the 15 -deg slope, the average total volume difference for each increased by an average of 1.8 times. The total volume difference for the 15-deg slope gap actually decreased by 15 percent because of the shadow created by the hydraulic lift mechanism on the RAVEN, which caused incorrect measurements of the terrain, as shown in Figure 14. When the 15-deg slope was measured from a standoff distance of $1.5 \mathrm{~m}$, the attachment apparatus shadow was not near the gap, and thus was not included in the volume subtraction.

The 3D subtraction volume plots also show areas where the terrain was rough. For example, lumps of soil are apparent on the edges in the 3D subtraction volume plots for Gap C (Figures 16-19). Photo 7 confirms the rough terrain of Gap C.

\section{Two-dimensional analyses}

The results of the 2D analyses of the slopes are presented in Figures 26-31. The 2D profiles for the three sets of LADAR data, labeled Laser A, B, and C, are plotted along with the total station data for comparison in Figures 46-87. Results of the 2D analyses are provided in Tables A2-A4. The average error for the three sets of LADAR data was computed for the total, approach, and departure groups of data. The shadow effect from the hydraulic lift mechanism is evident in some of the profiles. The LADAR sensor cannot measure the terrain because the lift mechanism is blocking the view.

Similar to the 3D analyses, the 2D analyses indicate that the LADAR system was effective for slopes up to 40 deg, with an average total error of $3.6 \mathrm{~cm}$. For each of the 2D profiles analyzed, the erroneous measurements caused by the hydraulic lift mechanism were not included in the accuracy assessment. The higher elevations at the approaching edge of the gap in Figure 47 are examples of these erroneous measurements. The average approach slope error for driver side, 
center, and passenger profiles at a standoff of $0 \mathrm{~m}$ was $3.7 \mathrm{~cm}$ at slopes up to $40 \mathrm{deg}$, and the standard deviation was $1.96 \mathrm{~cm}$. For the departure slope, the average error for slopes up to 40 deg at a standoff of $0 \mathrm{~m}$ was 3.5 , and the standard deviation was $2.07 \mathrm{~cm}$. The maximum error occurred at a slope of 15 deg.

When the vehicle is located at the edge of the gap, or standoff of $0 \mathrm{~m}$, the approach and departure slope errors are similar for slopes up to $40 \mathrm{deg}$; the maximum difference is $2.3 \mathrm{~cm}$. However, it is more difficult for the LADAR system to measure the slopes of 45 and $90 \mathrm{deg}$. The measurement error for the 45-deg approach slope is an average of four times greater than the 45-deg departure slope. The 90-deg approach slope could not be successfully measured with the LADAR system. Increasing the standoff distance to $1.5 \mathrm{~m}$ approximately doubled the error because the LADAR field of view did not completely cover the gap. These measurements for the approach slopes could be improved by increasing the field of view of the LADAR by increasing the height of the LADAR system or telescoping it toward the gap.

\section{Effect of Lighting}

Data were throughout the day at the following hours to evaluate the effect of lighting conditions on the LADAR system: 6, 7, 8, 9, 11, 13, 15, 17, 18, 19, 20, and 21. A sand gap with approach and departure slopes of approximately 15 deg was used for this testing. The RAVEN was not moved for the duration of the lighting experiment. Therefore, the LADAR data sets did not have to be registered with each other. The total station data were measured in the wheel paths and at the center of the RAVEN. Ambient lighting was quantified with a solar radiation sensor (LiCor LI200X), and was located at a nearby site within ERDC. The units for solar radiation are watts per square meter.

LADAR and total station data collected throughout the day are plotted in Figures 121-123. The measured profiles were similar, as shown in the graphs. The average error for the total gap was $4.5 \mathrm{~cm}$ for the driver side profile, $7.2 \mathrm{~cm}$ for the center profile, and $9.4 \mathrm{~cm}$ for the passenger side profile. The highest error values for each profile were approximately 12 percent higher than the average and occurred at times when the solar radiation was at its peak (hours 11 and 13), indicating that bright sunlight does have an effect on the LADAR accuracy (Figures 41-43). The standard deviations for all error values in this study were $0.43 \mathrm{~cm}, 0.41 \mathrm{~cm}$, and $0.40 \mathrm{~cm}$ for the driver side, center, and passenger side gap, respectively. The low standard deviation indicates that the data at each point for all hours were close to the same values.

\section{Effect of Surface Material}

The effect of various surface materials on LADAR measurements was evaluated by testing soil, sand, riprap $(3.81 \mathrm{~cm} \times 7.62 \mathrm{~cm}$ limestone), grass, gravel (No. 89 crushed limestone), and mud. The test site consisted of one large gap with a length of $24 \mathrm{~m}$ and width of $4 \mathrm{~m}$ that was separated into six sections 
with the same profile. Each section was covered with a different material (Figure 6). Images of the gaps are presented as Photos 9-19. The sand and soil gaps were compacted to provide smooth and incompressible surfaces. The mud, or wet soil, gaps were constructed by spraying water on the gaps (Photo 11). The approach and departure slopes were both approximately $15 \mathrm{deg}$. This slope was chosen to be a balance between slopes that were too large or small for LADAR measurements. It should be noted, however, that the highest errors for slopes under 40 deg occurred at a 15-deg slope.

Three sets of LADAR data and total station data were collected for the profiles at the wheel paths and the center of the vehicle. Because the mud gaps were the same gaps as the soil gaps, total station data collected for soil gaps were also used for the mud gaps.

The gap profiles for each material are shown in Figures 88-120. The LADAR profiles were similar to the total station profiles, indicating that these materials did not prevent LADAR measurement. In general, the total gap errors for the different materials in this study were grouped: mud and riprap had the lowest errors, sand and soil had slightly higher errors, and grass and gravel had the highest errors. The high error for the grass gap was anticipated. The LADAR was measuring the top of the grass blades, while the total station was measuring the actual ground. The high error for gravel probably results from the movement of the gravel during total station data collection. The mud, or wet soil, LADAR measurements had lower error values than those of the soil gaps. This is most likely because of increased reflectivity from the water.

Each material gap was tested under conditions of bright sunlight and shade, except for the sand gap, which was not in an area with shade. As with the previous study, the bright sunlight appears to have some effect on the error of the LADAR data (Figures 32-40). With the exception of mud, the error for the total gap in bright sunlight was approximately 19 percent larger than the error under shade.

\section{Effect of Dust and Fog}

The LADAR system was tested under fog/smoke and dust conditions. For comparison, LADAR data were taken at the same location without fog or dust. Fog was simulated using a fog machine at the lowest point of the gap (Photo 20). Dust was simulated using a leaf blower to blow silt across the gap (Photo 21).

Results for the fog and dust study are shown in Figures 44 and 45, respectively. It is evident that LADAR measurements are highly susceptible to error resulting from diffraction due to airborne particles. The LADAR could not penetrate the particles suspended in the air, resulting in inaccurate measurements of gap geometry. 


\section{Conclusions and Recommendations}

\section{Conclusions}

The 3D LADAR system developed by ASI was tested to assess the accuracy under a variety of conditions, including slopes, surface materials, light, fog/smoke, and dust. To measure the accuracy of the LADAR system, the terrain data were compared with total station surveyed data. The absolute value of the difference between the total station and LADAR data was used to quantify the error.

a. Data acquisition of a gap is much more efficient with the LADAR system. For a given gap, the manpower required to survey a gap is reduced from 2 to 1 using the LADAR system, the data acquisition time is shortened significantly, and the number of data points is greatly increased. Approximately 50,000 points were collected with the LADAR in $1 \mathrm{~min}$. In comparison, only 220 points were collected in a span of 30 min with the total station.

$b$. Both $3 \mathrm{D}$ and $2 \mathrm{D}$ analyses indicated that the LADAR system is effective for gaps with approach and departure slopes up to $40 \mathrm{deg}$ at a standoff distance of $0 \mathrm{~m}$. For slopes greater than $40 \mathrm{deg}$, the error approximately triples. At 90-deg approach slopes, the LADAR system is not feasible.

c. In the 3D analyses, the average total difference between LADAR and total station volumes was $0.95 \mathrm{~m}^{3}$ for approach slopes up to 40 deg at a 0 -m standoff distance. This value increased to $3.43 \mathrm{~m}^{3}$ for a 45 -deg approach slope. In the 2D analyses, the average error for gaps with approach slopes up to 40 deg was $3.6 \mathrm{~cm}$. The error significantly increases for an approach slope of 45 deg to $10.96 \mathrm{~cm}$.

d. Increasing the standoff distance from $0 \mathrm{~m}$ to $1.5 \mathrm{~m}$ approximately doubles the error of the LADAR data because the LADAR field of view of the gap is limited. Thus, it is imperative that the sensor be positioned as close to the gap as possible.

e. Light did not have a significant effect on the LADAR measurements. At 1100 and $1300 \mathrm{hr}$, when the solar radiation was highest, the errors were 
slightly higher (approximately 12 percent) than the average. Overall, the measurements were consistent throughout the day. The overall standard deviation value for all 2D error measurements for the light study was $0.4 \mathrm{~cm}$.

f. The LADAR measurements are influenced by material types. However, no apparent trend could be discerned. The total gap 2D average error for all materials ranged from $2.3 \mathrm{~cm}$ to $12.8 \mathrm{~cm}$ in bright sunlight and $2.2 \mathrm{~cm}$ to $11.1 \mathrm{~cm}$ under shade. The lowest errors were observed for mud and riprap. Higher errors were determined for sand, soil, gravel, and grass. LADAR profiles for the materials tested during this investigation had similar slopes to the total station profiles, indicating all of the materials were suitable for evaluation using the LADAR system.

g. The LADAR system was adversely affected by particles in the air, such as fog, smoke, or dust. The LADAR system could not penetrate the suspended particles, and thus returned false terrain measurements.

h. Although the total station was used as the ground truth for comparison in this study, it should be noted that total station measurements were also susceptible to error. Possible sources of error could come from allowing the prism rod to sink into the surface and not holding the rod completely still and level.

i. A systematic error was observed in all data sets, causing the average error to increase by $2 \mathrm{~cm}$ from the driver side profile to the center profile, and from the center profile to the passenger profile. It is suspected that the error results from the LADAR sensor not being level. This can be corrected by calibrating the LADAR system.

\section{Recommendations}

The following recommendations are based upon the results and conclusions of this investigation.

a. The LADAR system is affected when the system is not at the edge of a gap and when the system is too close to the gap because the hydraulic lift mechanism on the RAVEN blocks the field of view. Therefore, the system should be modified so that it is mounted such that a clear field of view of the gap can be detected with the LADAR system.

$b$. To correct the tilt in the data, the LADAR system should be calibrated on a regular basis.

c. Mobius $^{\circledR}$, the software used to control the LADAR system, should be simplified to make the system more user friendly. Preset parameters should be defined so pressing one button is all that is required to acquire LADAR data at a gap. 
d. Fog, smoke, and dust all adversely affect LADAR measurements. Alternative solutions and algorithms for these conditions are needed. Other ranging sensors could be used in these situations, or algorithms such as the last pulse method should be developed for the LADAR sensor to overcome the particles in the air. 


\section{References}

Barshan, B., and Aytaç, T. (2003). "Position-invariant surface recognition and localization using infrared sensors,” Opt. Eng. 42(12), 3589-94.

Benet, G., Blanes, F., Simó, J. E., and Pérez, P. (2002). “Using infrared sensors for distance measurement in mobile robots,” Robotics and Autonomous Systems 40, 255-66.

Borenstein, J., and Koren, Y. (1988). “Obstacle avoidance with ultrasonic sensors,” IEEE Journal of Robotics and Automation 4(2), 213-18.

Cheok, G. S., and Stone, W. C. (2004). "Performance evaluation facility for LADARs," Proceedings of SPIE. Laser Radar Technology and Applications IX, April 13-15, 2004. 54-65.

Elta Electronics Industries, Ltd. (1996). “Elta ${ }^{\circledR} 40$ R/Elta ${ }^{\circledR}$ 50R Recording Total Stations Operating Instructions. Ashdod, Israel.

Fernández, J., Grau, A., and Martínez, A. B. (1999). "Vision based obstacle detection for autonomous industrial vehicles,” Proceedings, Emerging Technologies and Factory Automation. 7th IEEE International Conference, October 18-21, 1999. 167-172.

Foessel-Bunting, A., Bares, J., and Whittaker, W. (2001). “Three-dimensional map building with MMW radar,” Proceedings, 3rd International Conference on Field and Service Robotics, Yleisjäljennös-Painnopörssi, Helsinki, Finland, June 2001.

García, E., and Lamela, H. (2001). "Low-cost 3D vision system based on a low power semiconductor laser rangefinder and a single scanning mirror,” Opt. Eng. 40(1), 61-66.

Grantham, J. W., and Meidunas, E. C. (1998). "Laser radar in adverse weather,” SPIE Conference on Laser Radar Technology and Applications III, Orlando, FL, April 14-16, 1998. 303-14.

Hebert, M. (2000). “Active and passive range sensing for robotics,” Proceedings, 2000 International Conference on Robotics and Automation, San Francisco, CA, April 2000. 102-10.

Novotny, P. M., and Ferrier, N. J. (1999). "Using infrared sensors and the phong illumination model to measure distances," IEEE International Conference on Robotics and Automation. Detroit, MI, May 10-15, 1999. 1644-49. 
SICK AG. (2002). “Technical Description: Laser Measurement Systems. Waldkirch, Germany.

Wijesoma W. S., Kodagoda, K. R. S., and Balasuriya, A. P. (2004). "Road-boundary detection and tracking using ladar sensing," IEEE Transactions on Robotics and Automation 20(3), 456-64. 

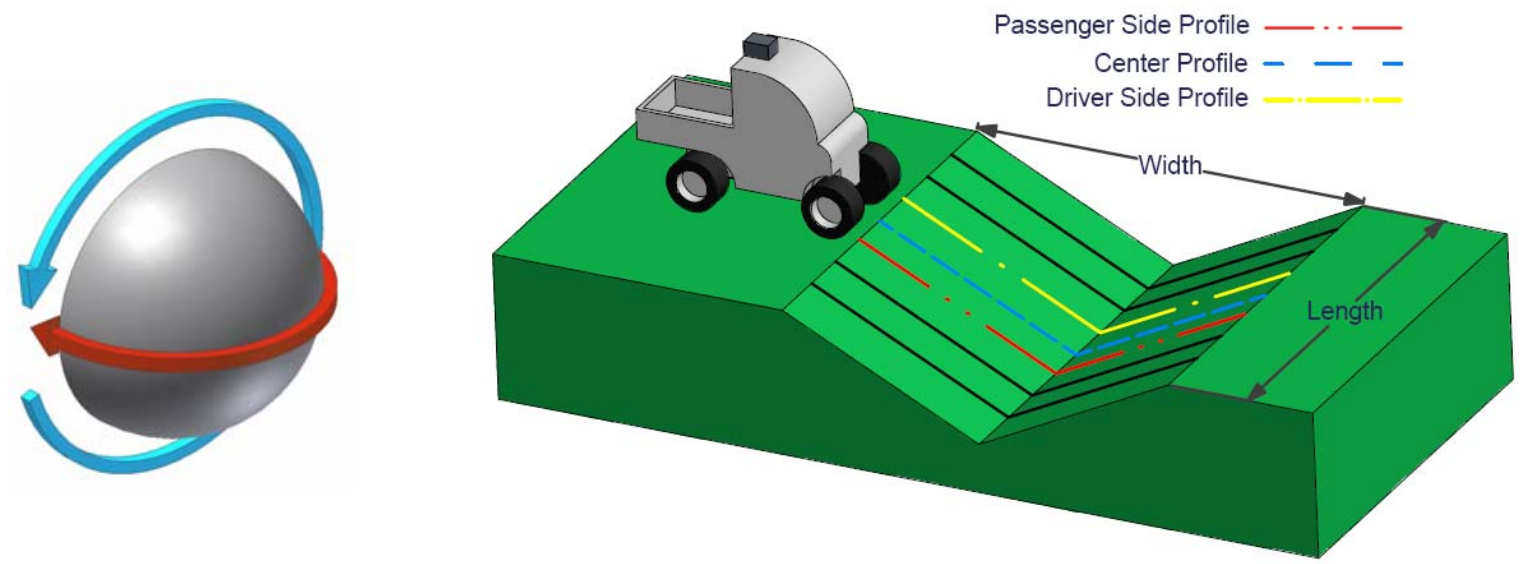

Figure 1. (A, left) Hemispherical sensing area for the LADAR system. The red arrow represents the SICK LMS sensor's 180-deg sweep and the blue arrow represents 360-deg rotation of the sensor. (B, right) Length, width, and 2D profiles of a gap.
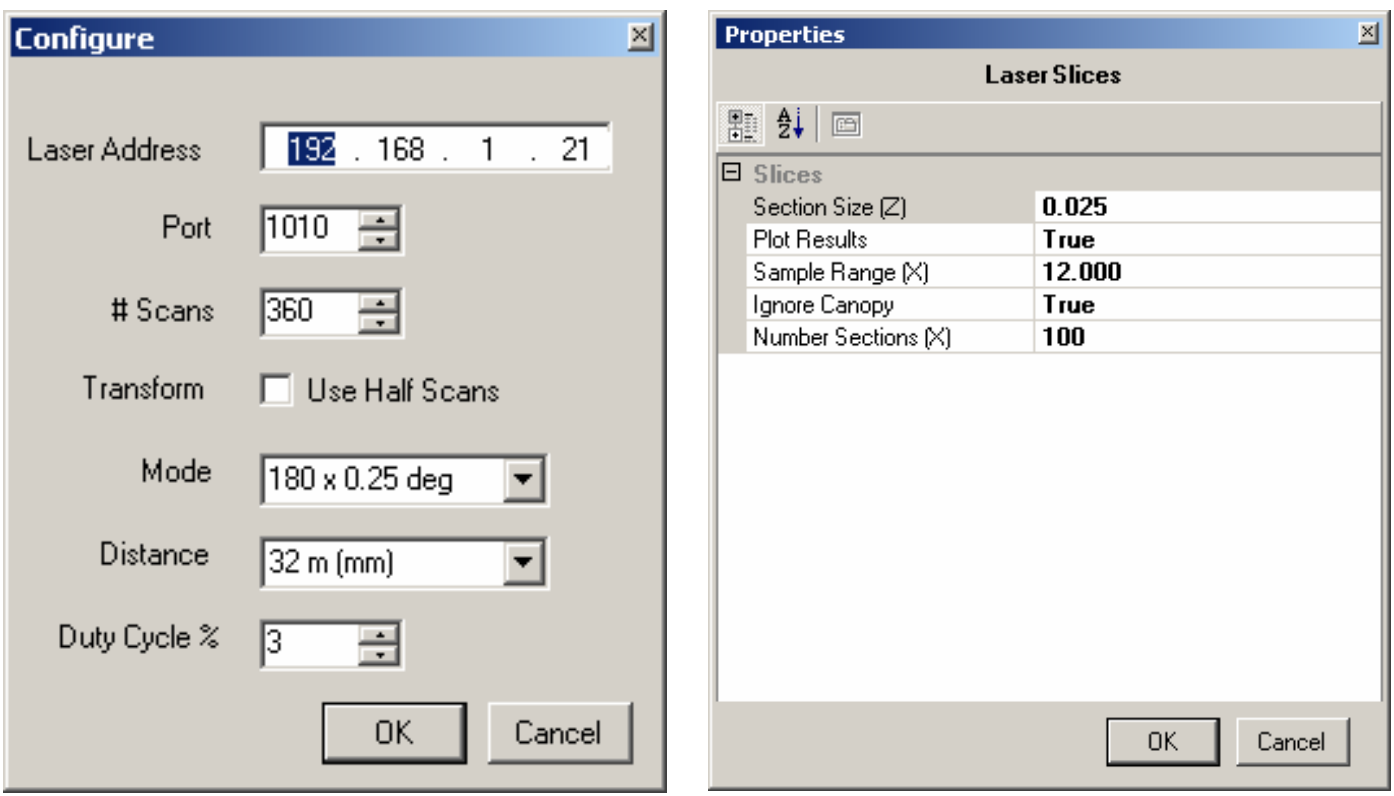

Figure 2. Setting menus in Mobius ${ }^{\circledR}$ used to control the LADAR system. 


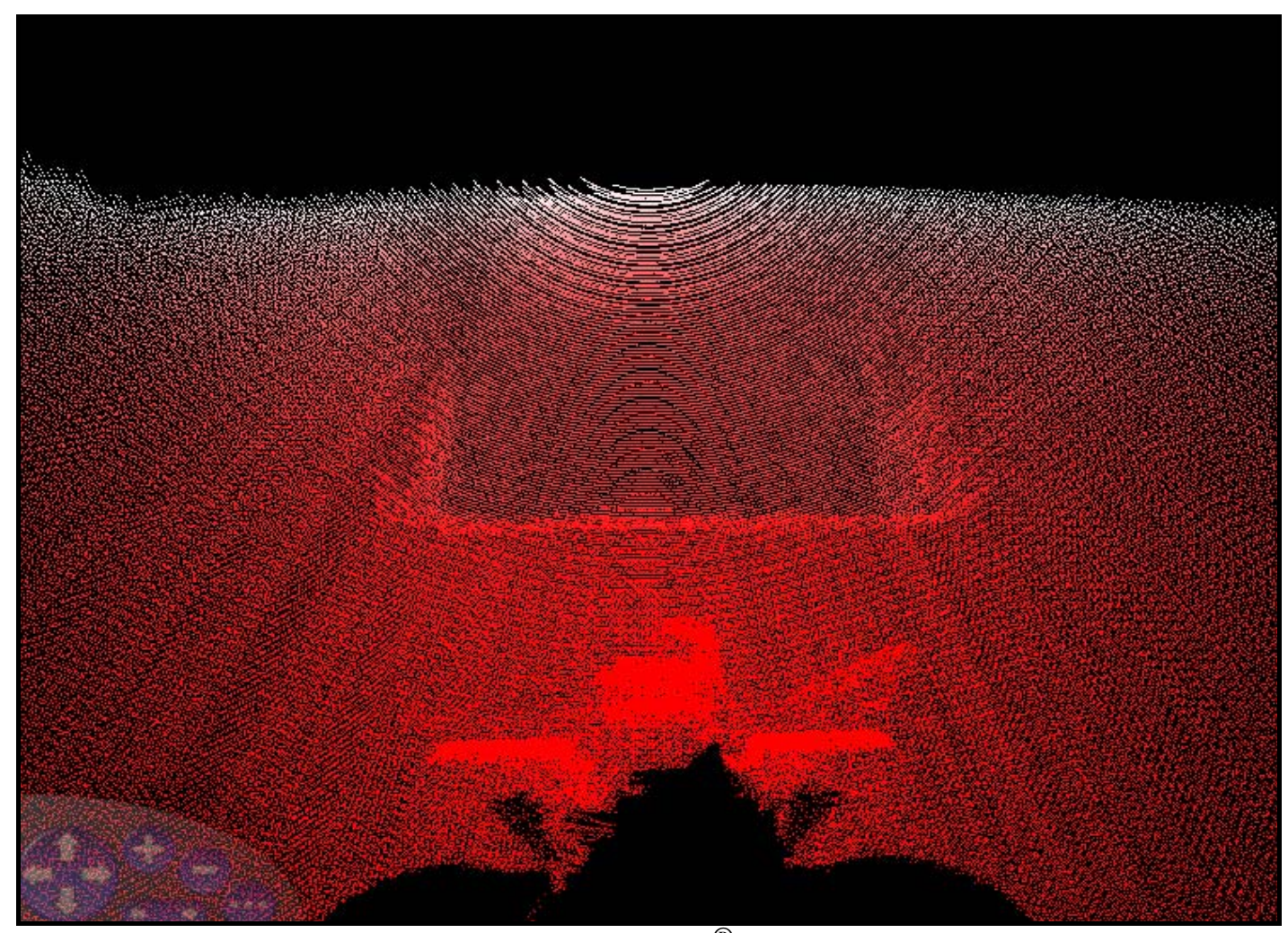

Figure 3. 3D point cloud display of a gap in Mobius ${ }^{\circledR}$ (front view of the gap).

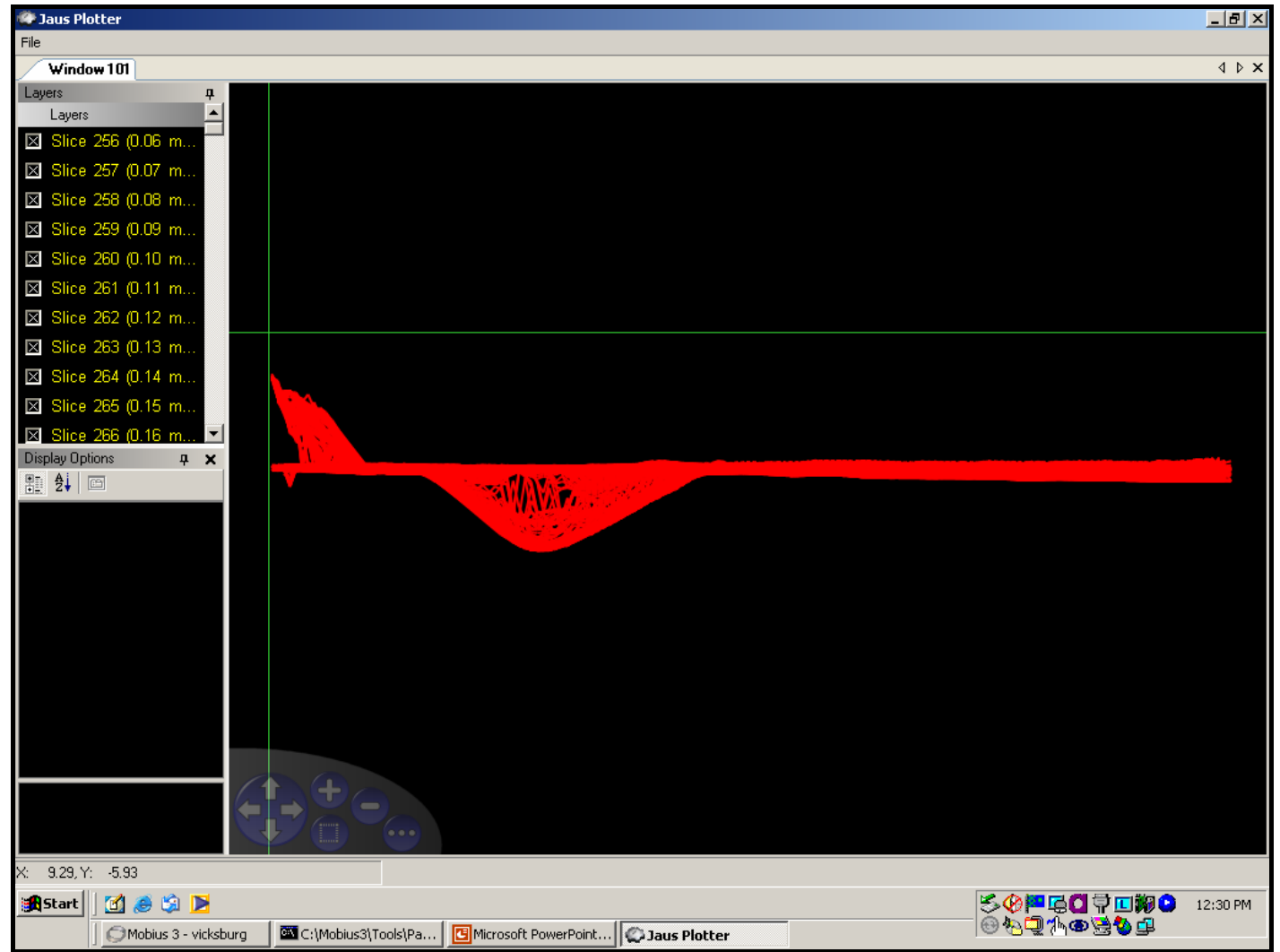

Figure 4. 2D profiles of a gap in Jaus plotter (side view of the gap). 


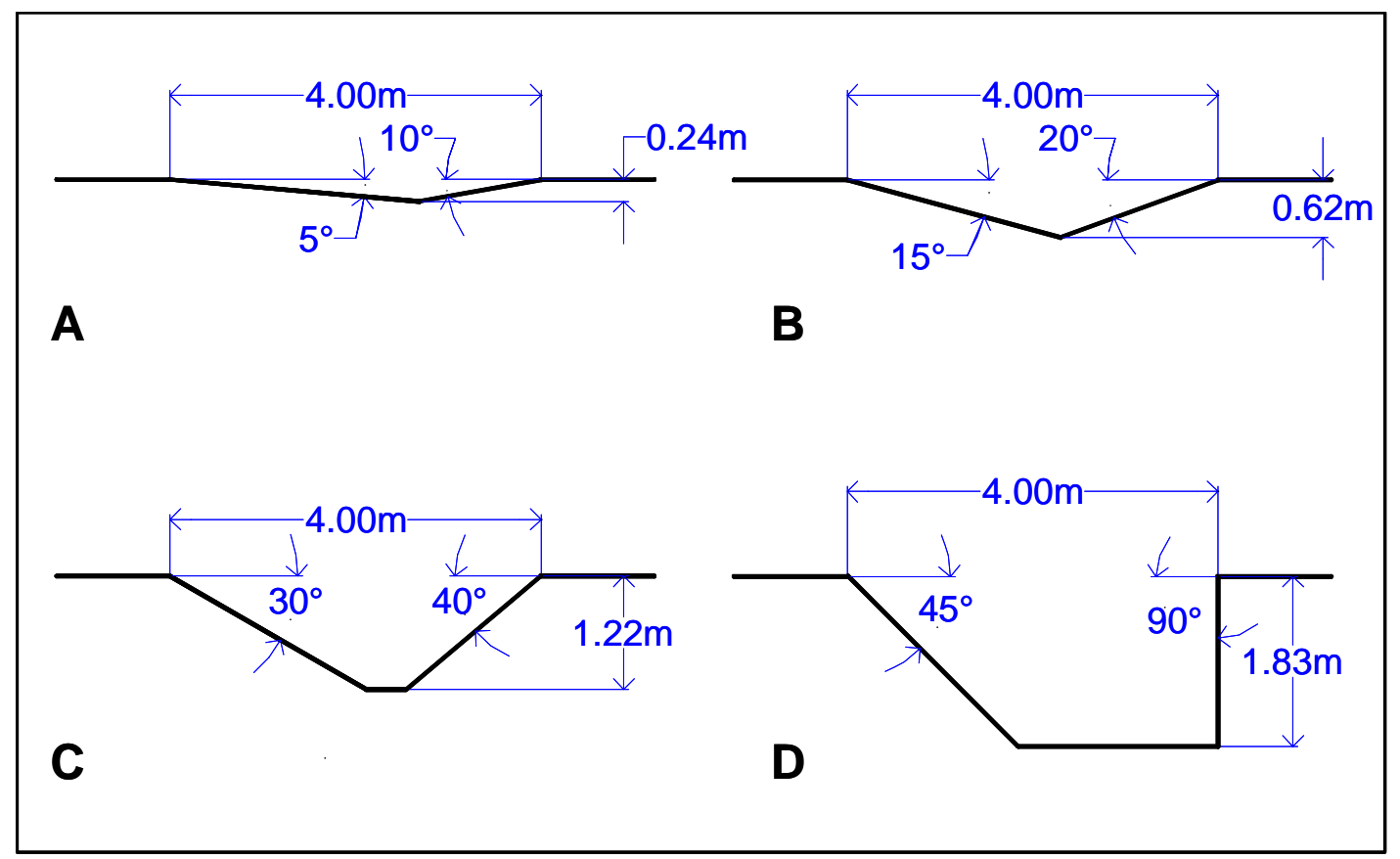

Figure 5. Side view (profiles) of gaps constructed to examine the effect of slopes on LADAR data.

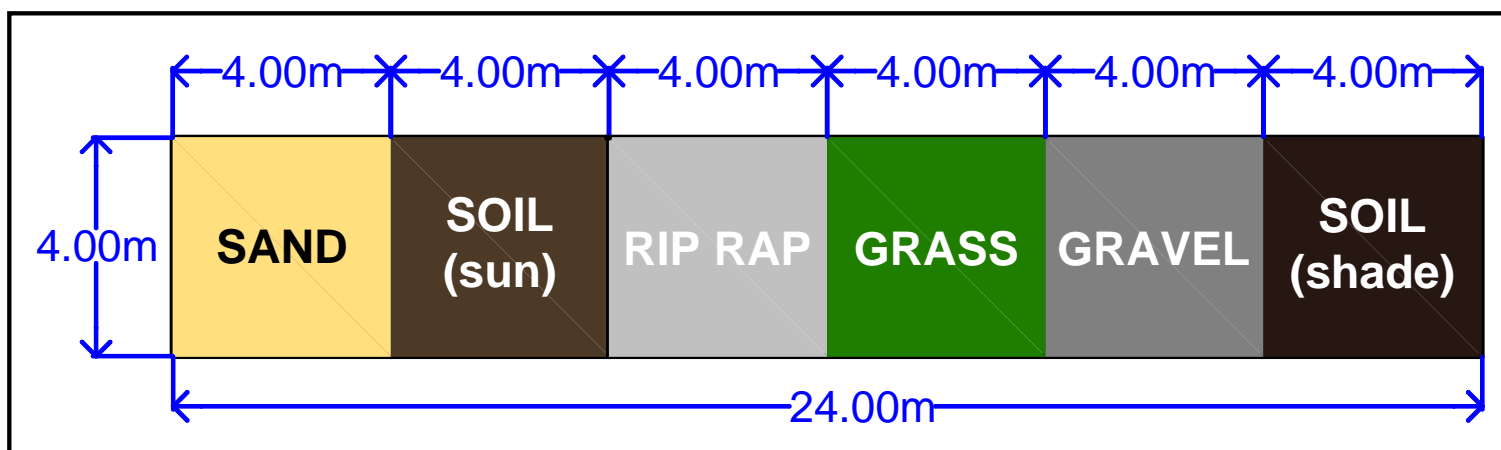

Figure 6. Top view of gap constructed to examine the effect of surface materials of LADAR data. The approach and departure slopes were approximately $15 \mathrm{deg}$. 


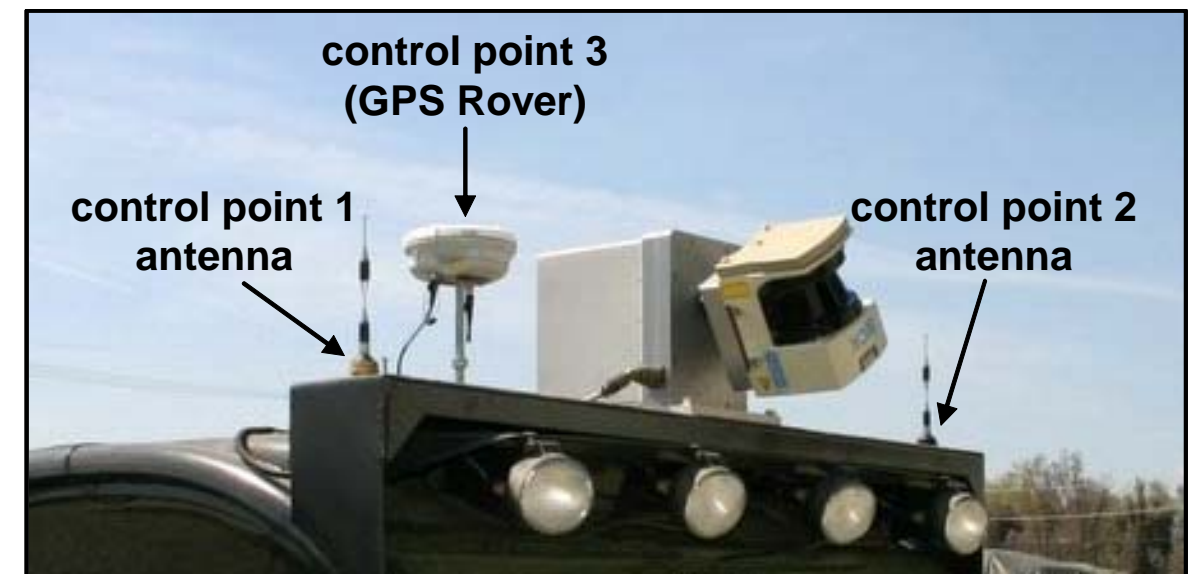

Figure 7. Control points used to register total station data with LADAR data.

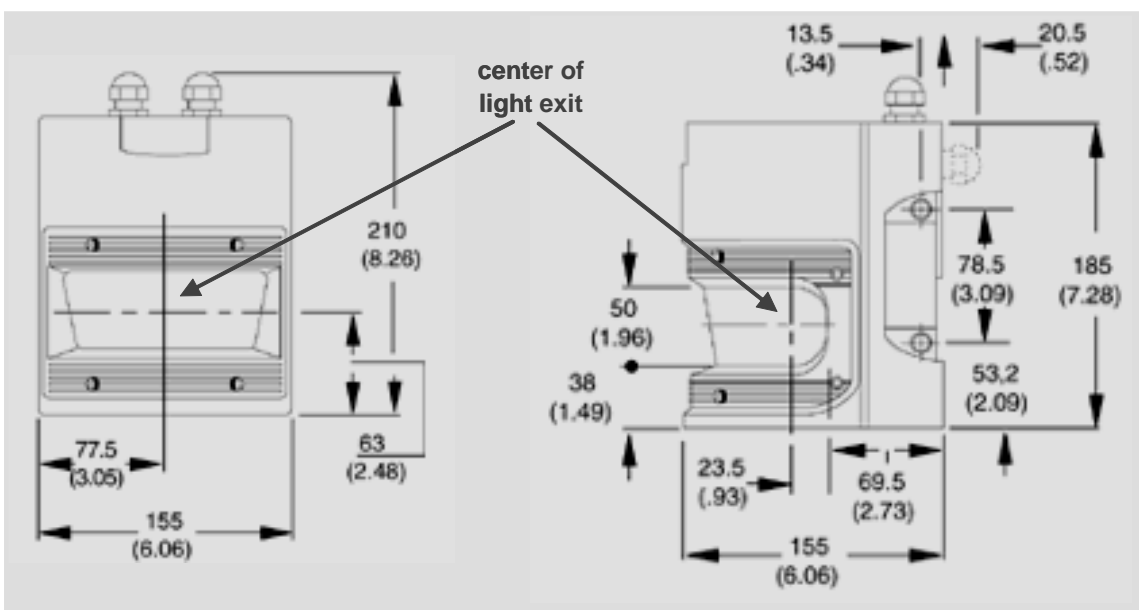

Figure 8. Location of the origin of the SICK LMS sensor; the origin is the point at which the SICK LMS sensor rotates.

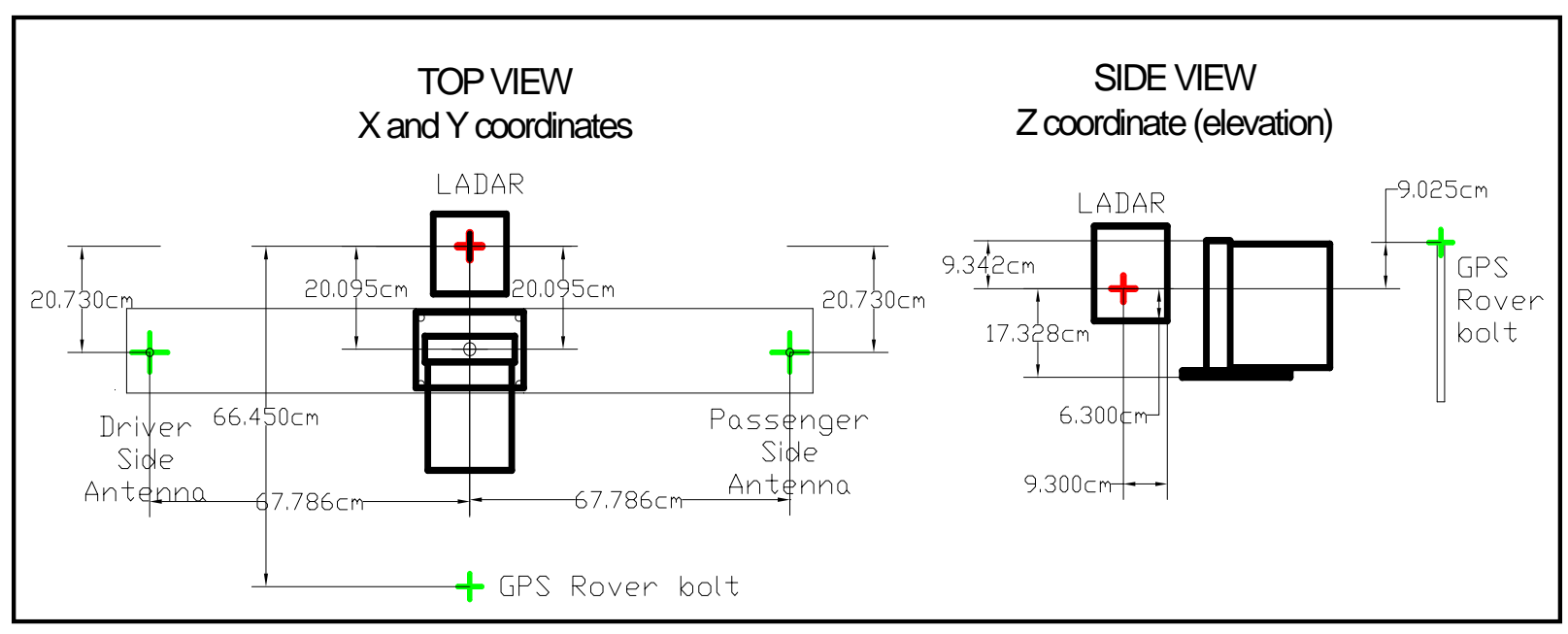

Figure 9. Location of control points with respect to the center of the SICK LMS Laser sensor. 


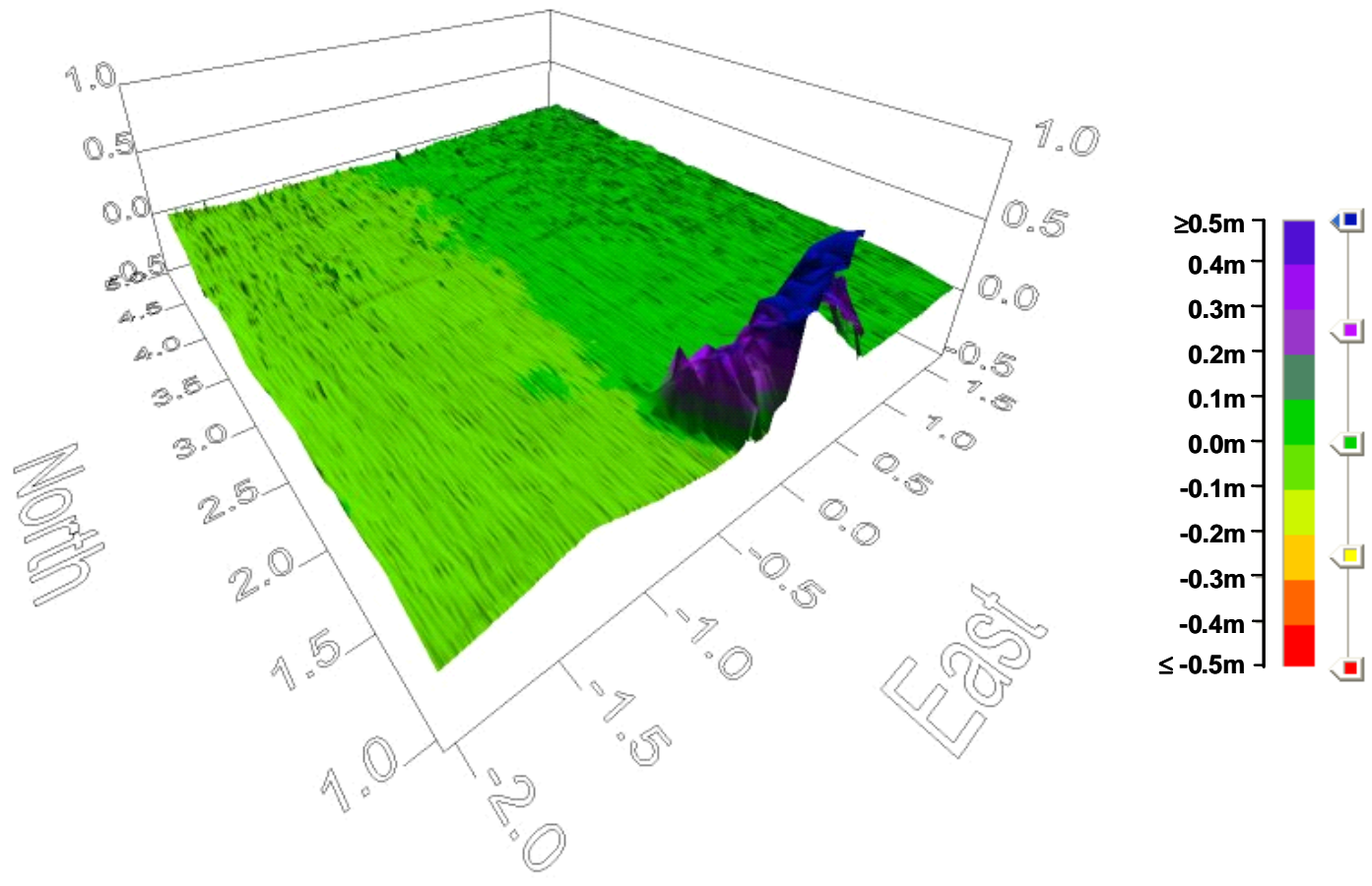

Figure 10. 3D volume difference results for Gap A with an approach slope of 5 deg, departure slope of $10 \mathrm{deg}$, and standoff distance of $0 \mathrm{~m}$. Graph is plotted in meters.

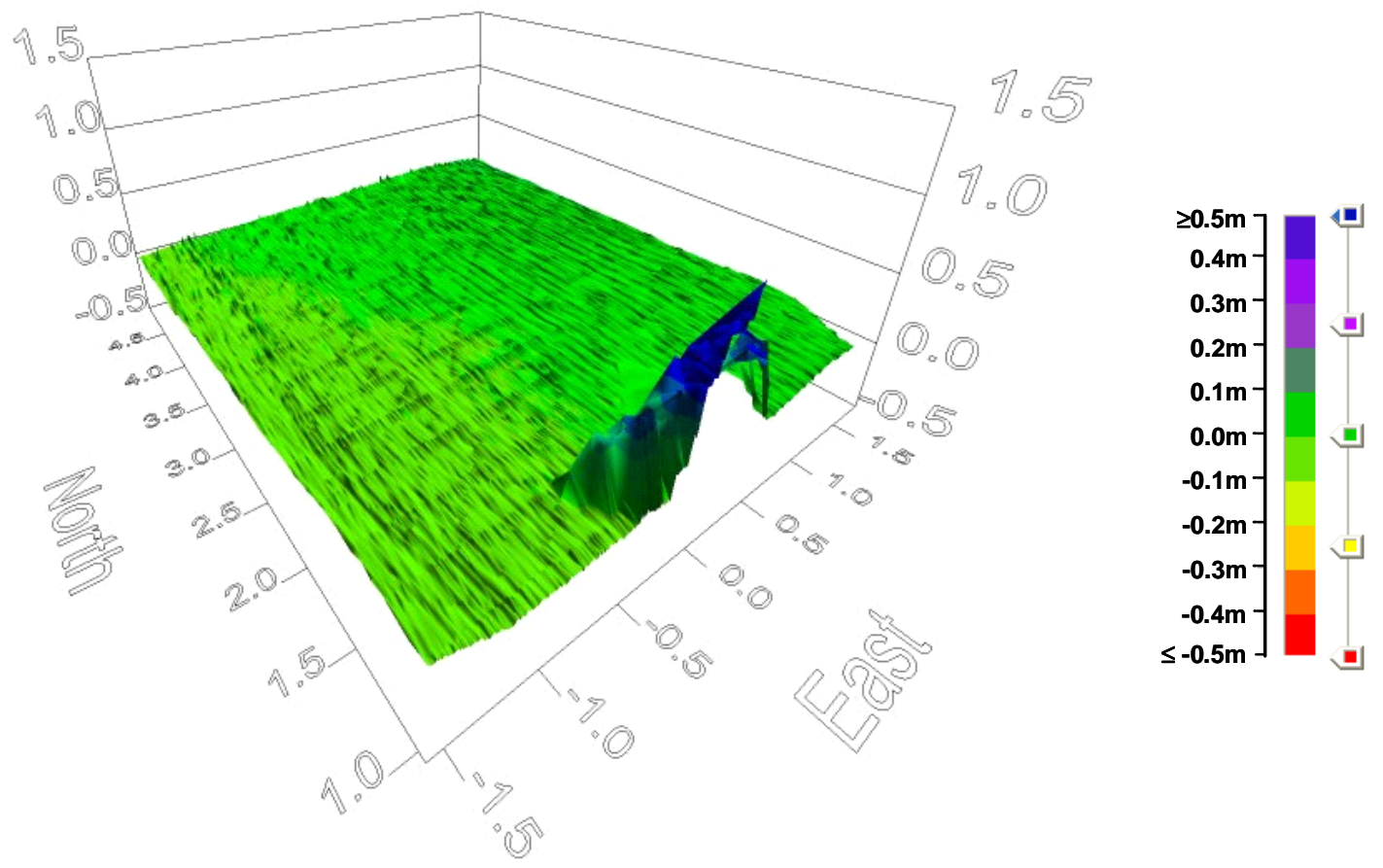

Figure 11. 3D volume difference results for Gap A with an approach slope of $10 \mathrm{deg}$, departure slope of $5 \mathrm{deg}$, and standoff distance of $0 \mathrm{~m}$. Graph is plotted in meters. 


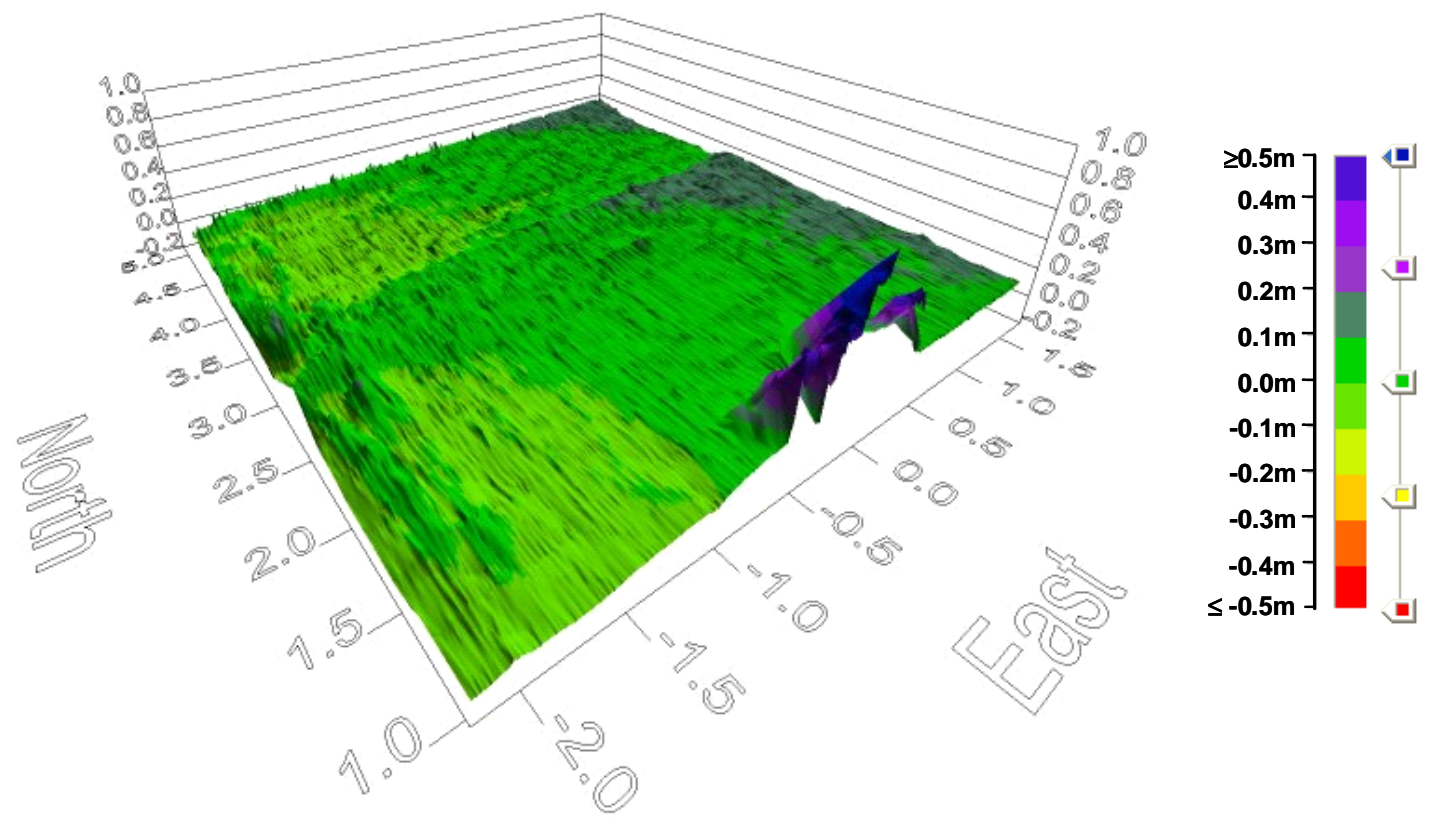

Figure 12. 3D volume difference results for Gap B with an approach slope of 15 deg, departure slope of $20 \mathrm{deg}$, and standoff distance of $0 \mathrm{~m}$. Graph is plotted in meters.

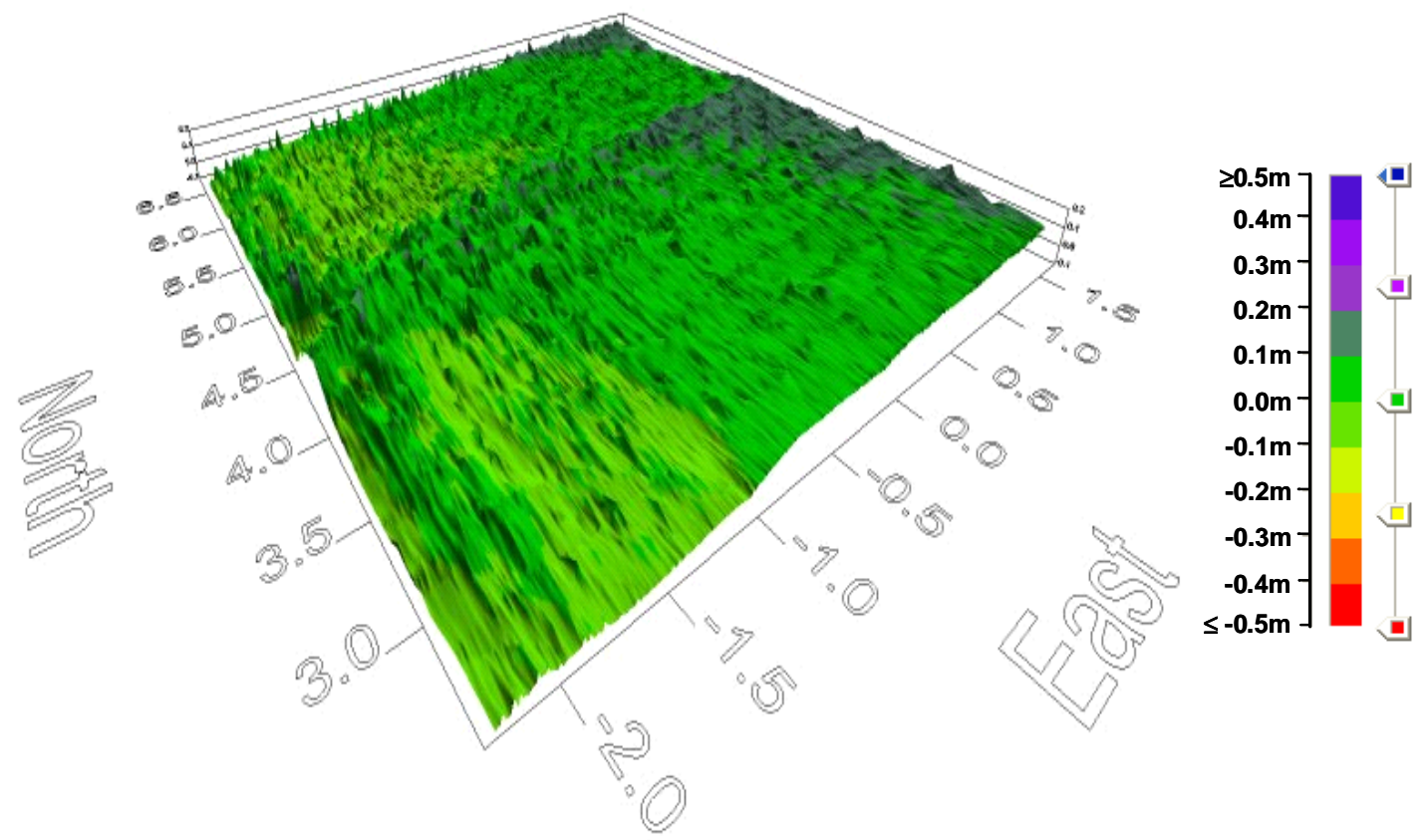

Figure 13. 3D volume difference results for Gap B with an approach slope of 15 deg, departure slope of $20 \mathrm{deg}$, and standoff distance of $1.5 \mathrm{~m}$. Graph is plotted in meters. 


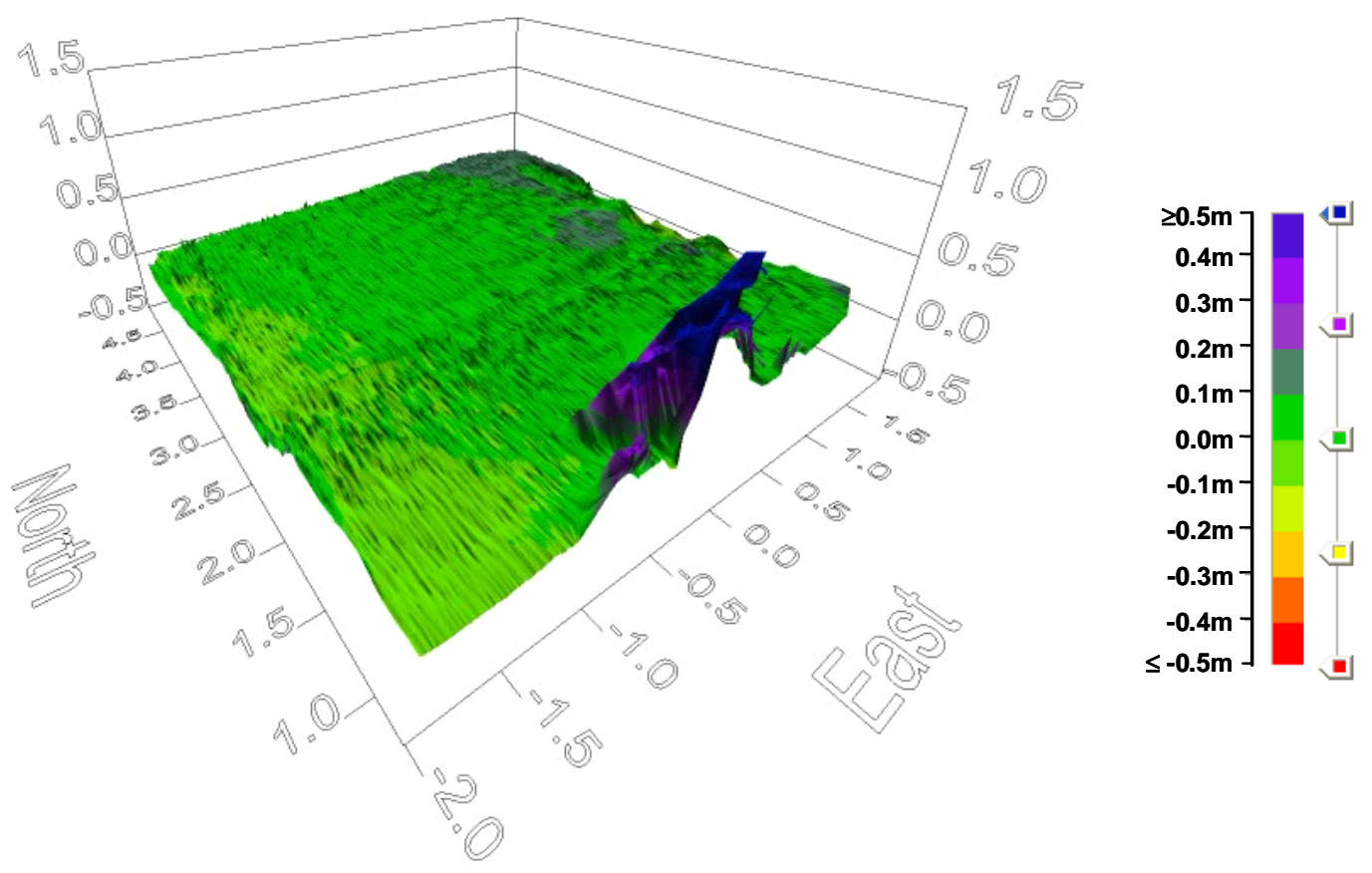

Figure 14. 3D volume difference results for Gap B with an approach slope of 20 deg, departure slope of $15 \mathrm{deg}$, and standoff distance of $0 \mathrm{~m}$. Graph is plotted in meters.

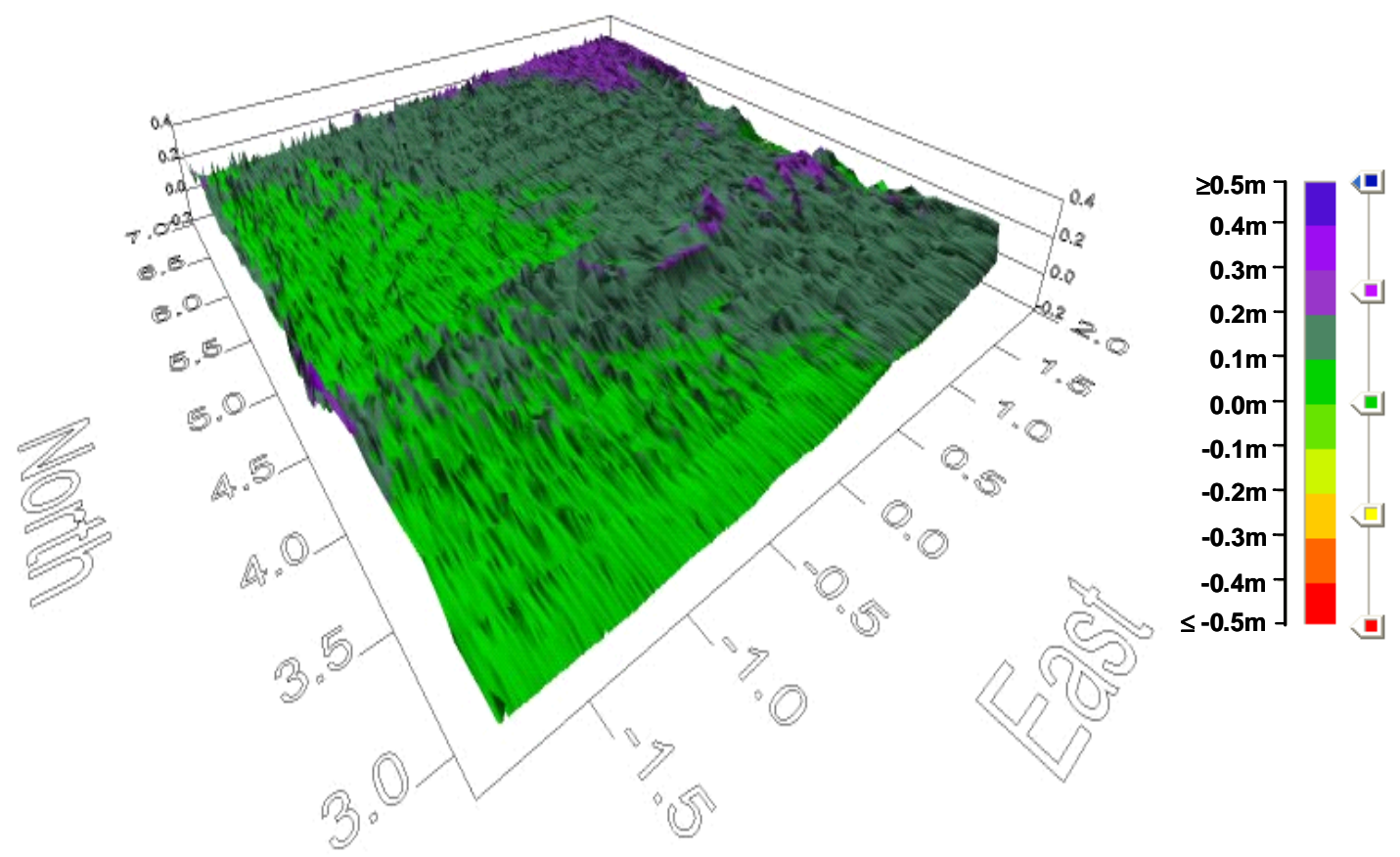

Figure 15. 3D volume difference results for Gap B with an approach slope of 20 deg, departure slope of $15 \mathrm{deg}$, and standoff distance of $1.5 \mathrm{~m}$. Graph is plotted in meters. 


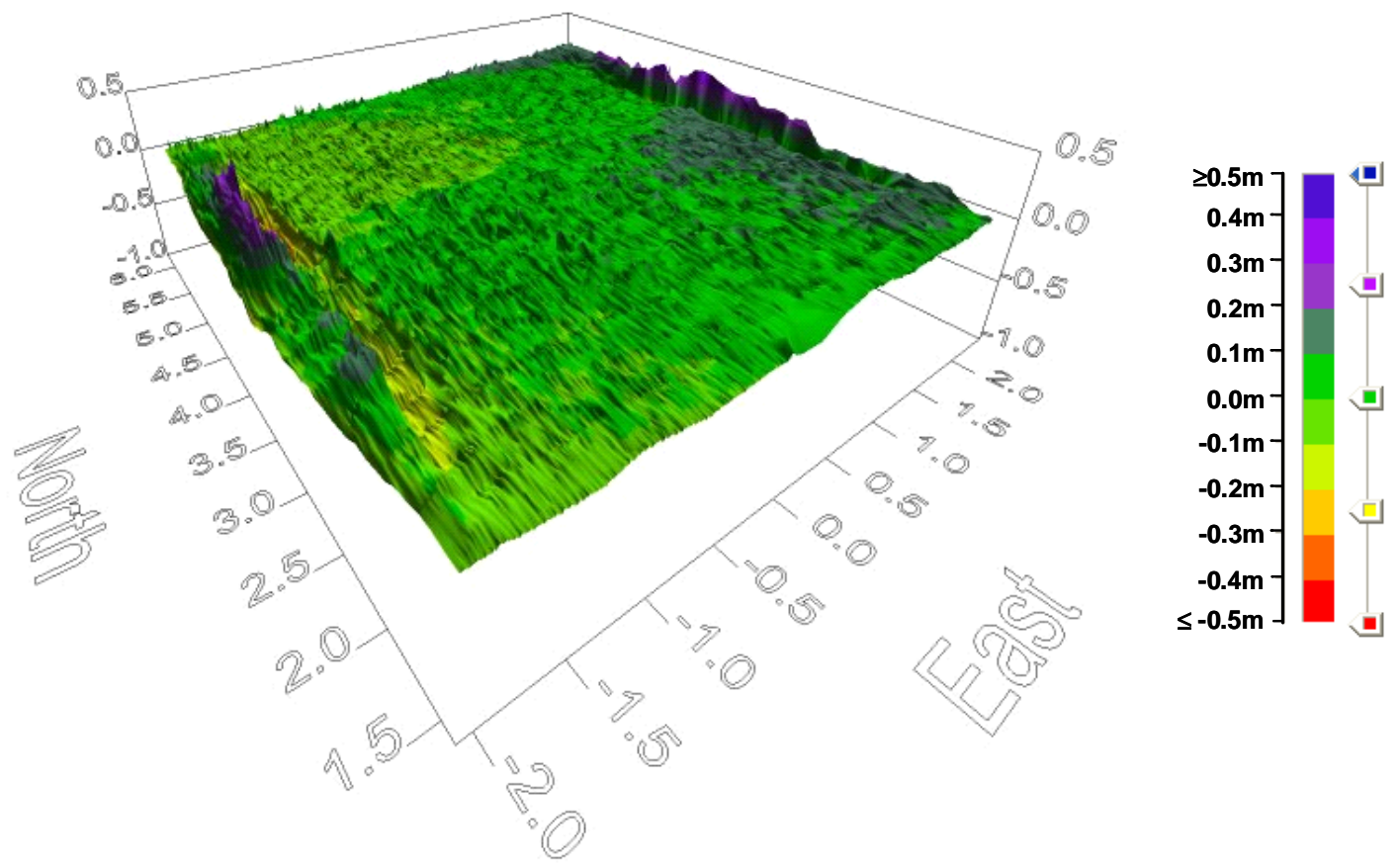

Figure 16. 3D volume difference results for Gap C with an approach slope of $30 \mathrm{deg}$, departure slope of $40 \mathrm{deg}$, and standoff distance of $0 \mathrm{~m}$. Graph is plotted in meters.

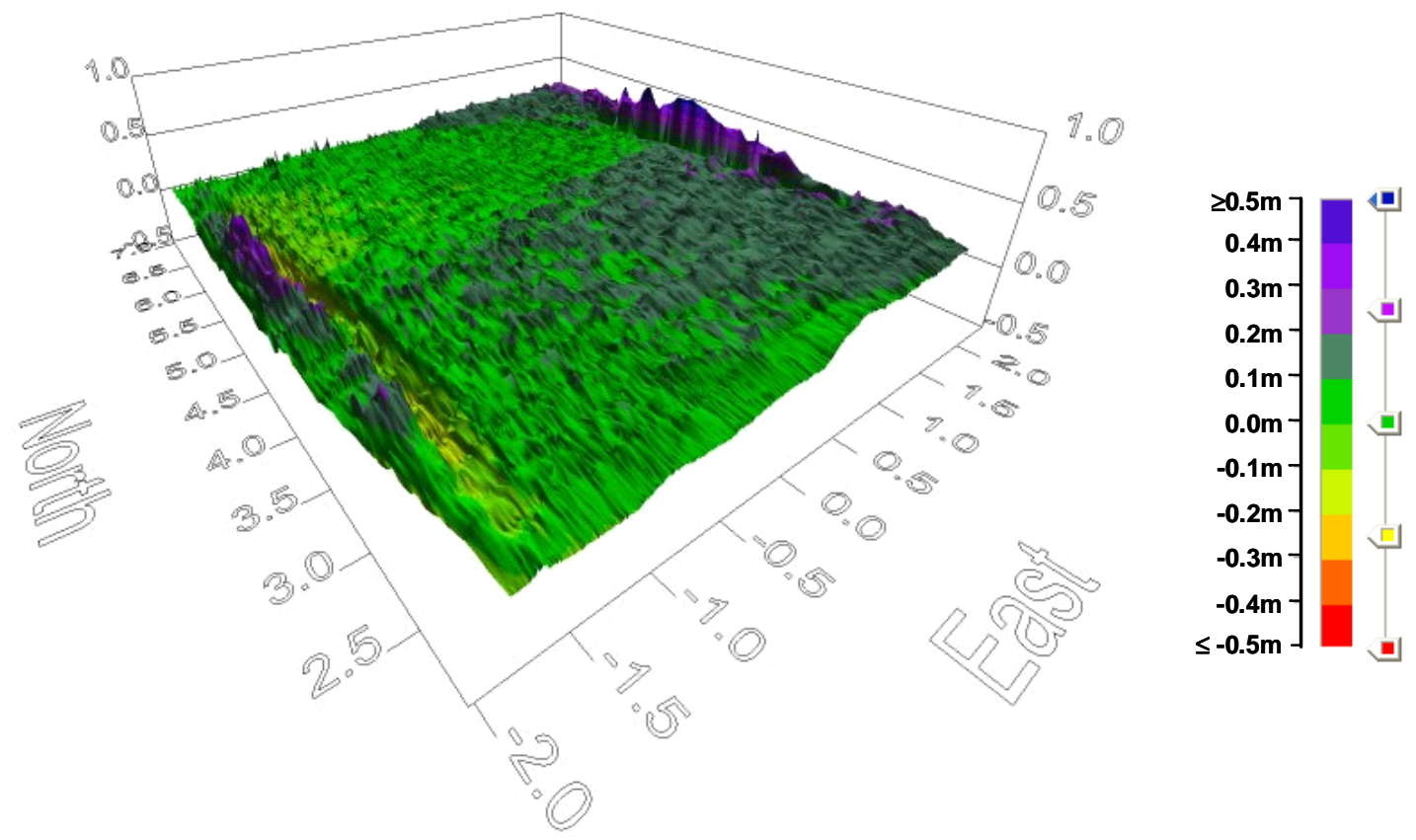

Figure 17. 3D volume difference results for Gap C with an approach slope of $30 \mathrm{deg}$, departure slope of $40 \mathrm{deg}$, and standoff distance of $1.5 \mathrm{~m}$. Graph is plotted in meters. 


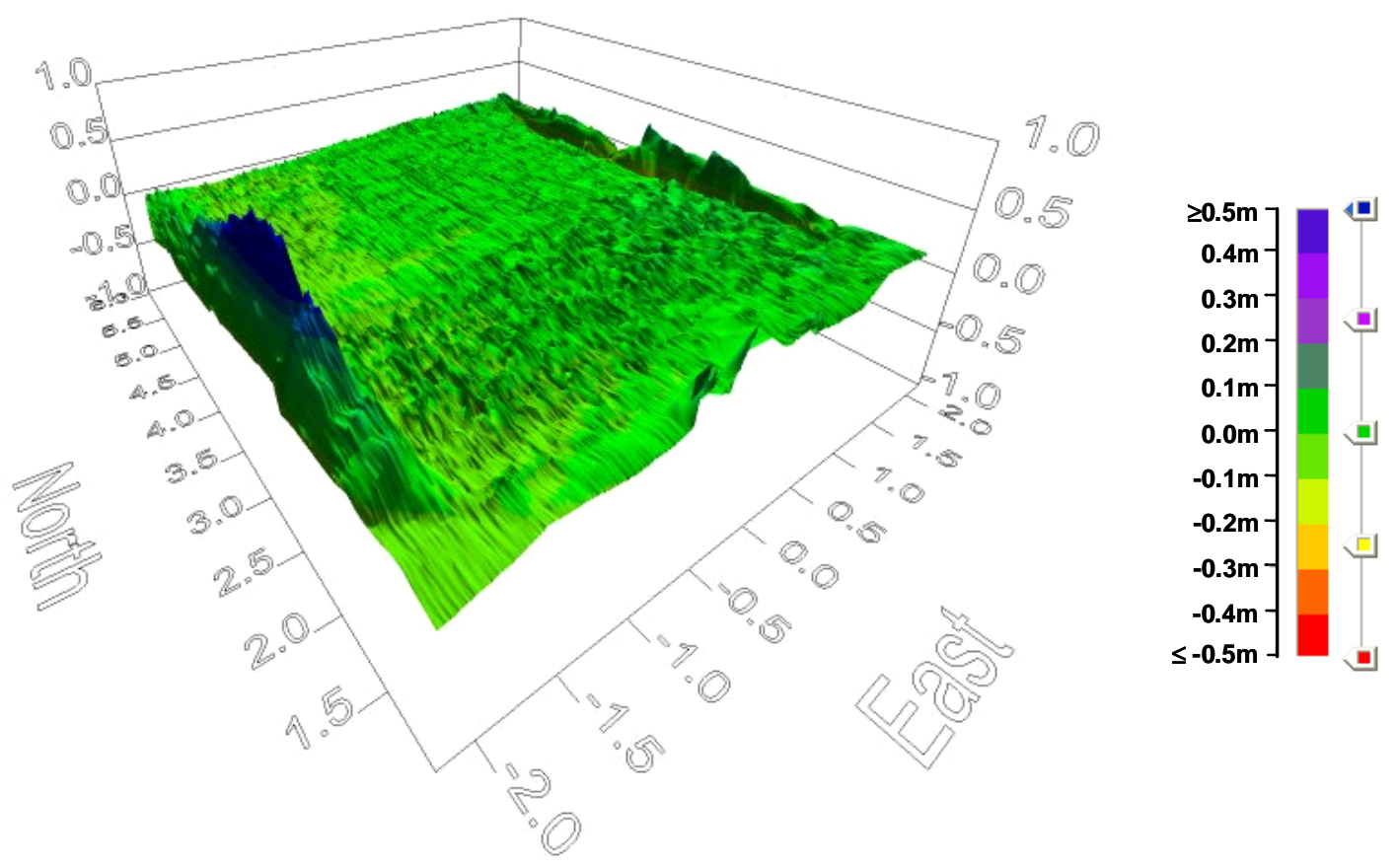

Figure 18. 3D volume difference results for Gap C with an approach slope of $40 \mathrm{deg}$, departure slope of $30 \mathrm{deg}$, and standoff distance of $0 \mathrm{~m}$. Graph is plotted in meters.

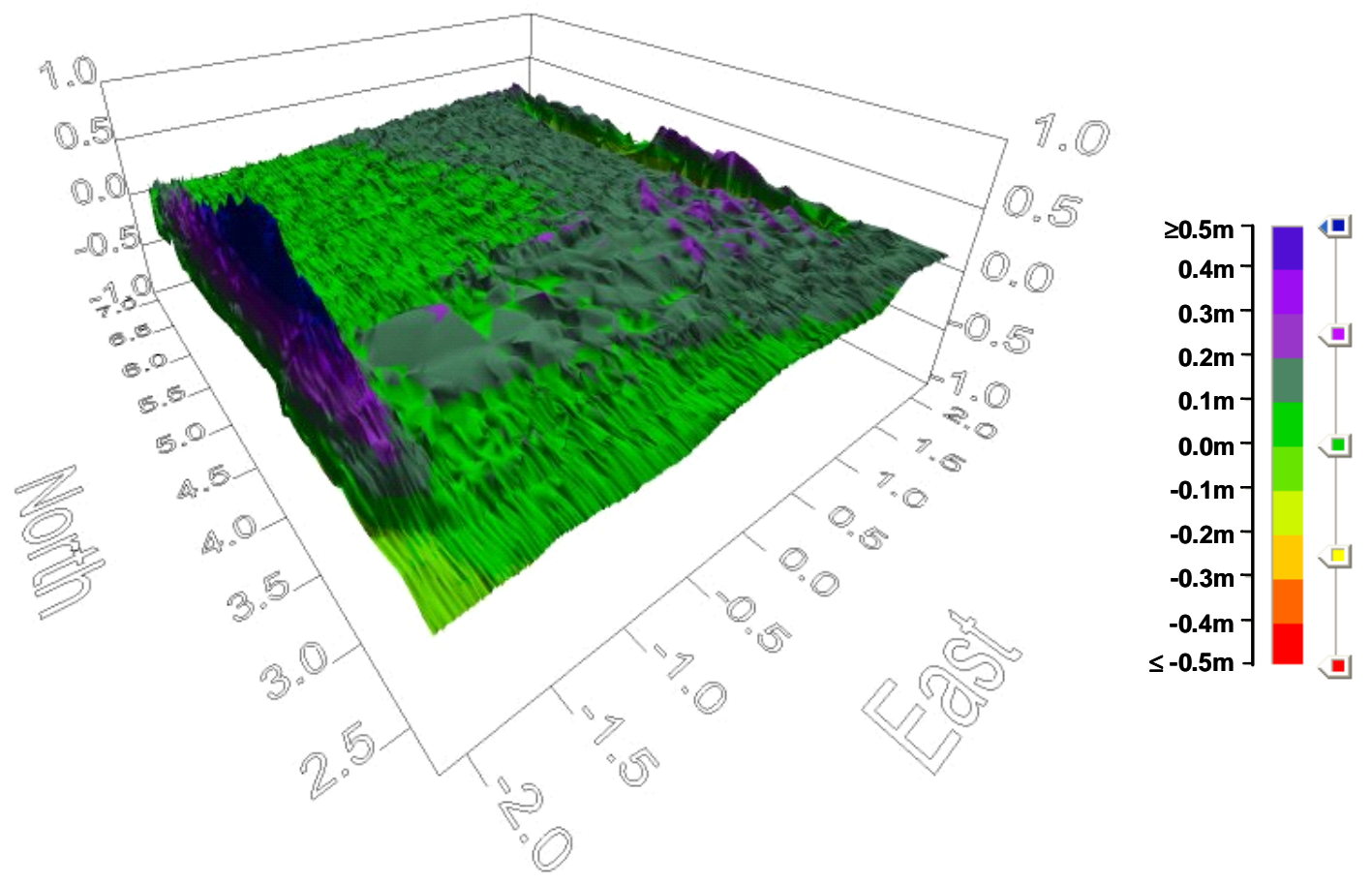

Figure 19. 3D volume difference results for Gap C with an approach slope of $40 \mathrm{deg}$, departure slope of $30 \mathrm{deg}$, and standoff distance of $1.5 \mathrm{~m}$. Graph is plotted in meters. 


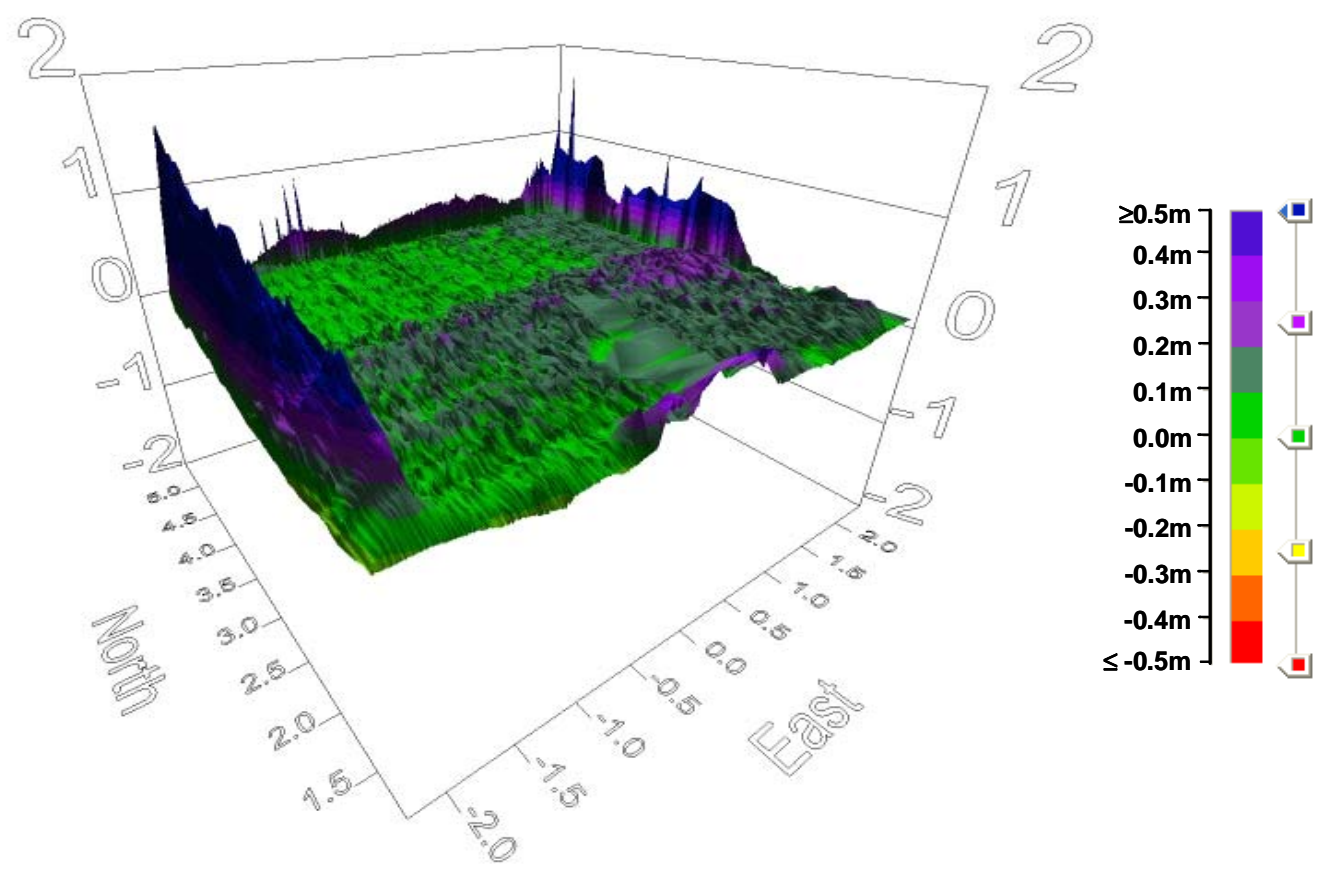

Figure 20. 3D volume difference results for Gap D with an approach slope of $45 \mathrm{deg}$, departure slope of $90 \mathrm{deg}$, and standoff distance of $0 \mathrm{~m}$. Graph is plotted in meters.

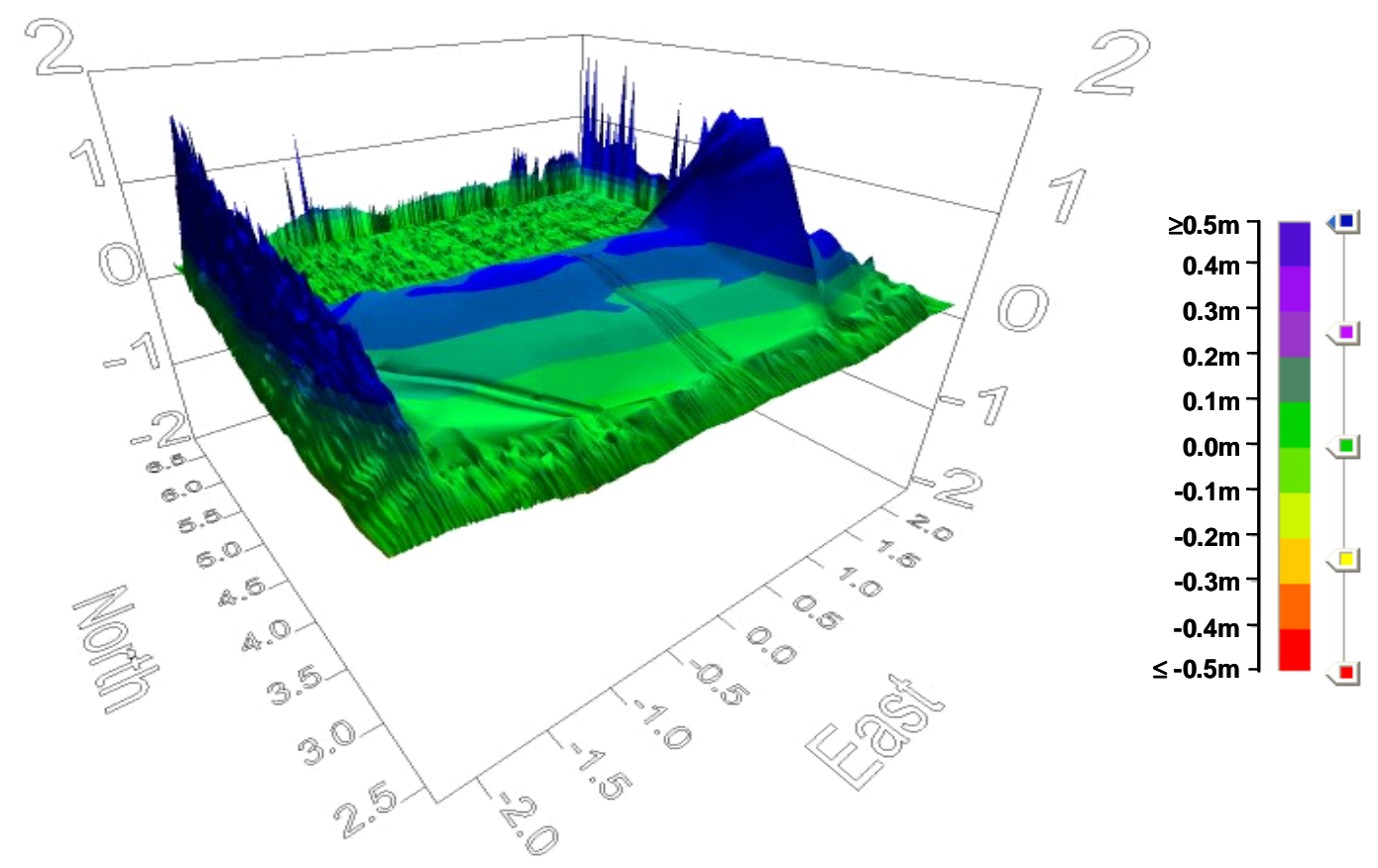

Figure 21. 3D volume difference results for Gap D with an approach slope of $45 \mathrm{deg}$, departure slope of $90 \mathrm{deg}$, and standoff distance of $1.5 \mathrm{~m}$. Graph is plotted in meters. 


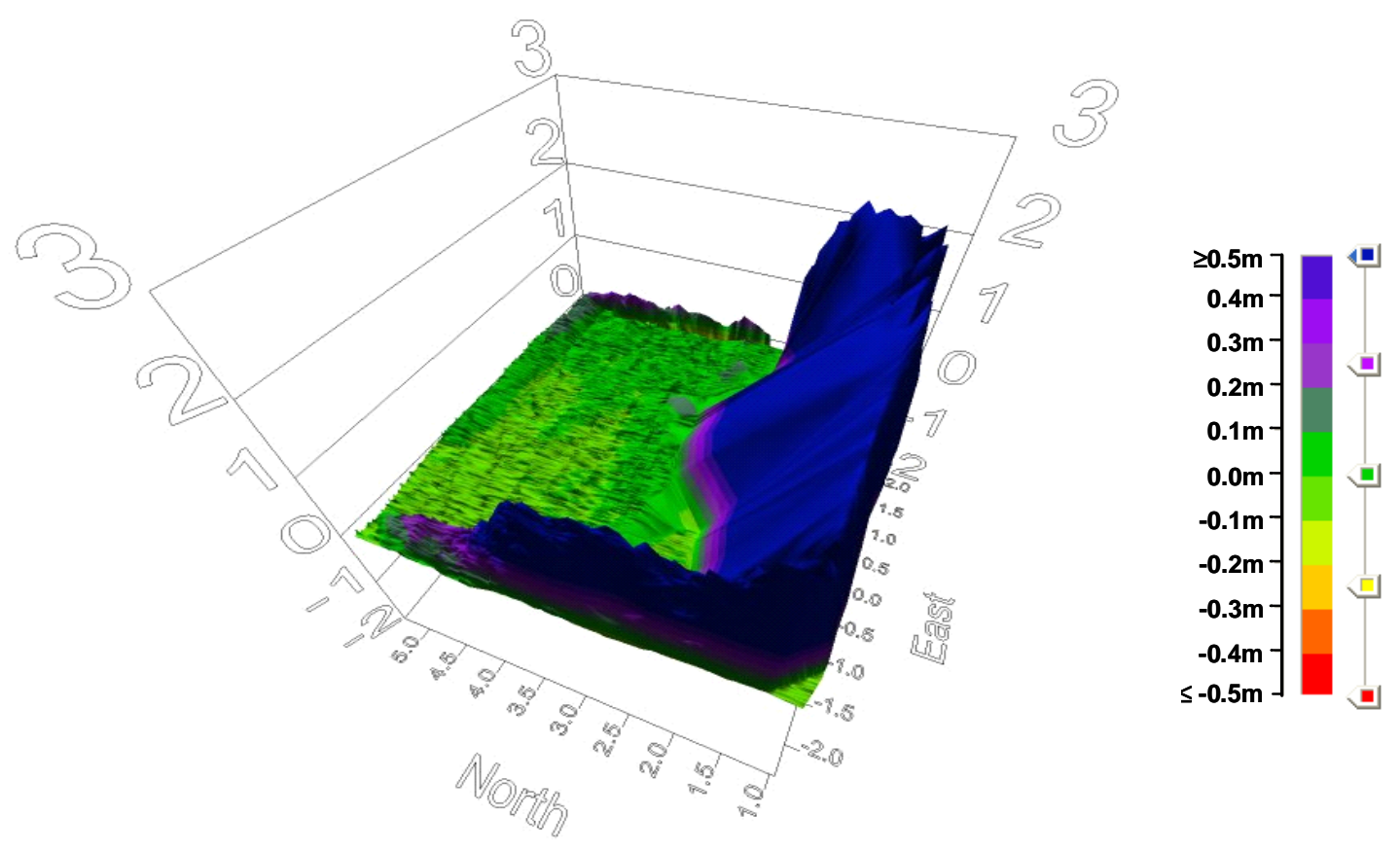

Figure 22. 3D volume difference results for Gap D with an approach slope of 90 deg, departure slope of $45 \mathrm{deg}$, and standoff distance of $0 \mathrm{~m}$. Graph is plotted in meters.

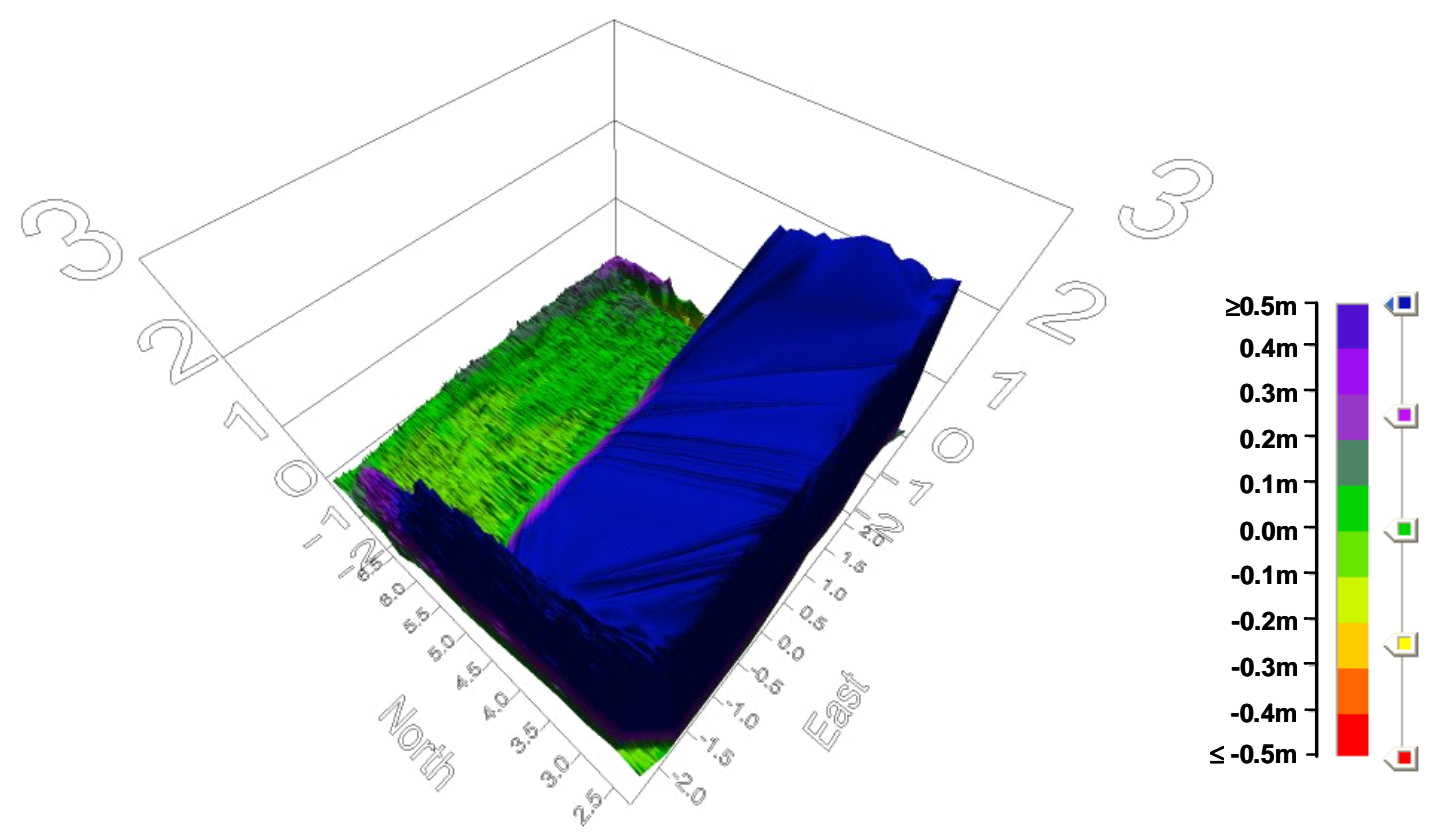

Figure 23. 3D volume difference results for Gap D with an approach slope of $90 \mathrm{deg}$, departure slope of $45 \mathrm{deg}$, and standoff distance of $1.5 \mathrm{~m}$. Graph is plotted in meters. 


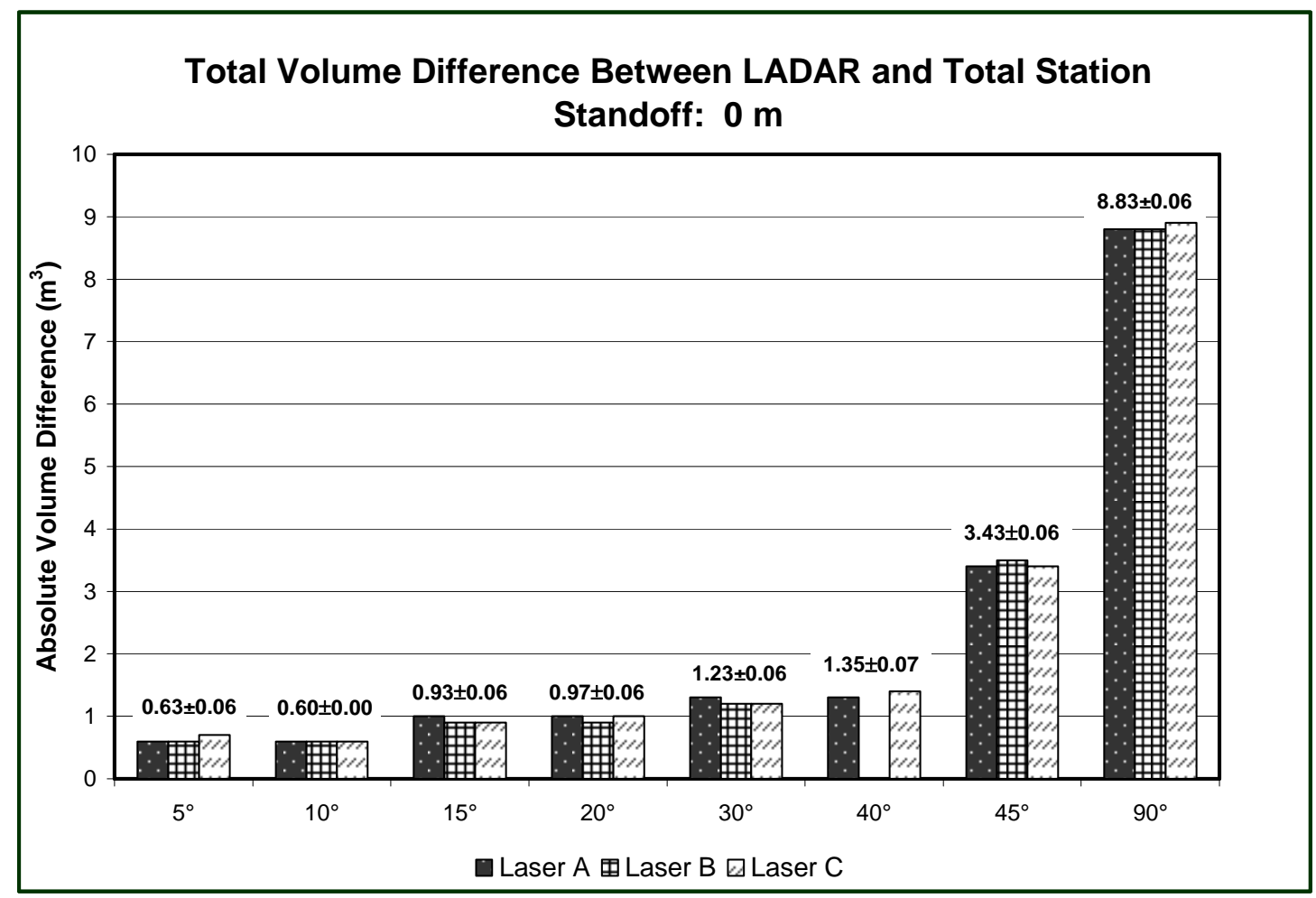

Figure 24. Subtraction of LADAR and total station volumes at a standoff distance of $0 \mathrm{~m}$. Average and standard deviation are reported above each set of data.

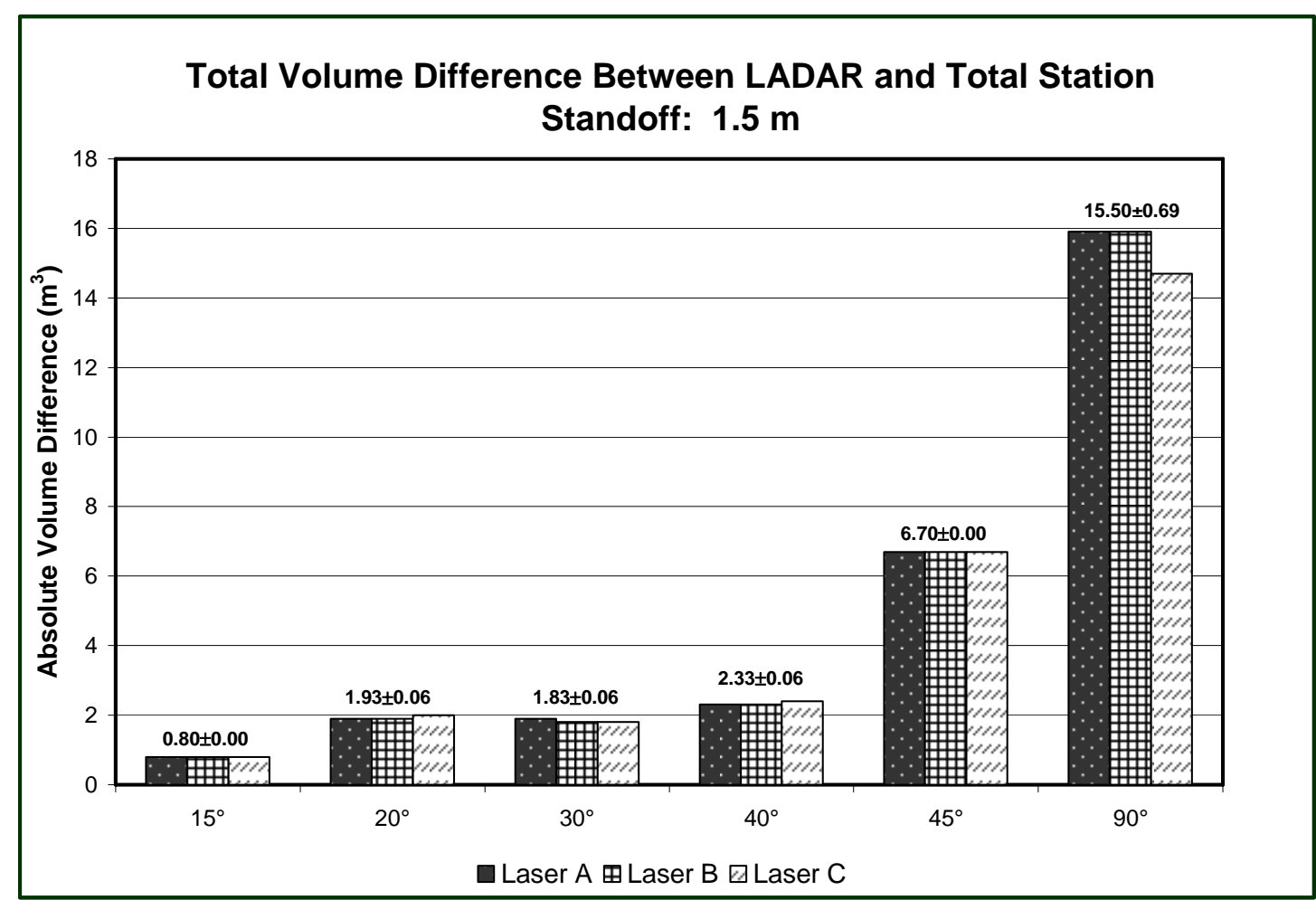

Figure 25. Difference of LADAR and total station volumes at a standoff distance of $1.5 \mathrm{~m}$. Average and standard deviation are reported above each set of data. 


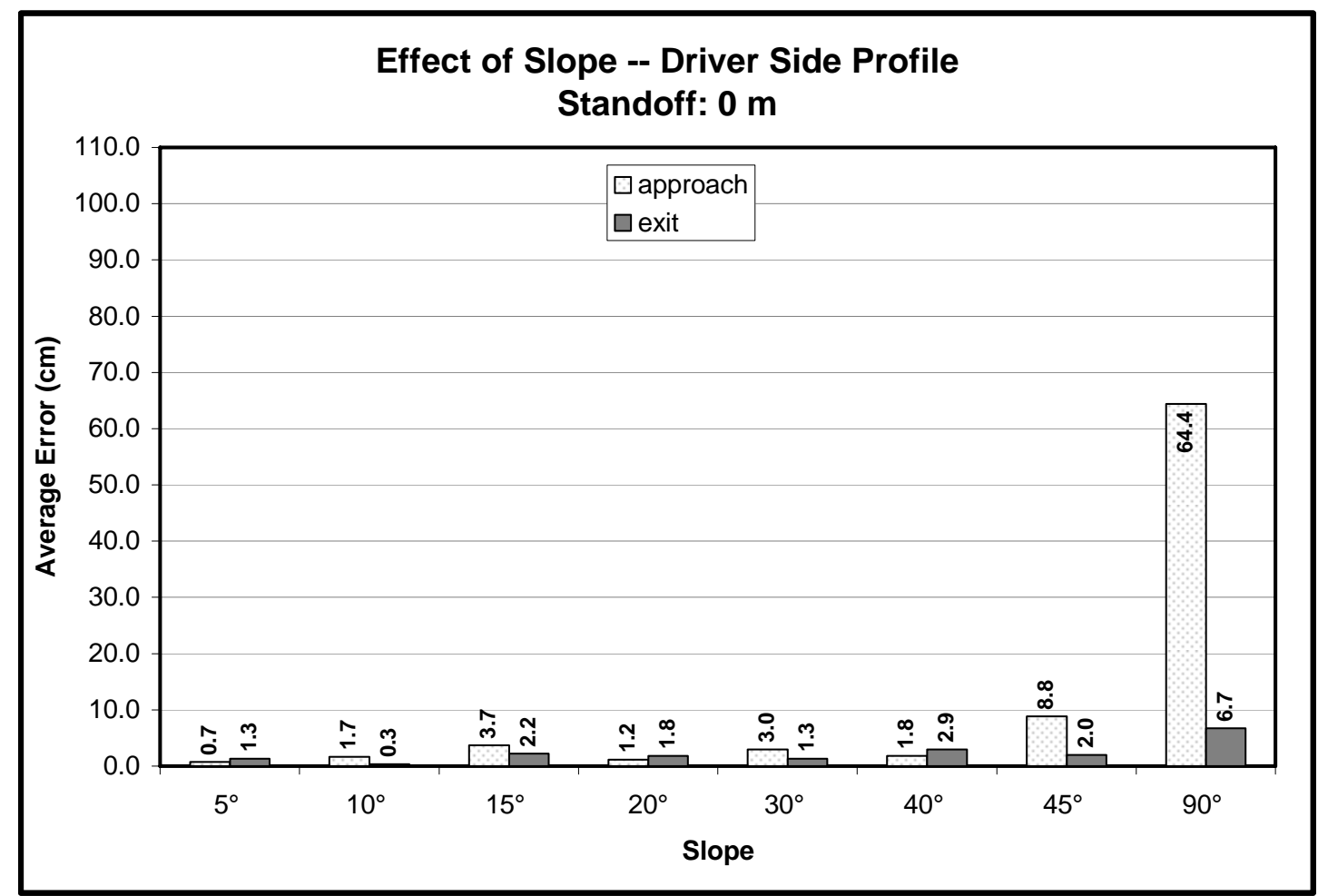

Figure 26. Average error at various slopes from a standoff of $0 \mathrm{~m}$ for the driver side profile.

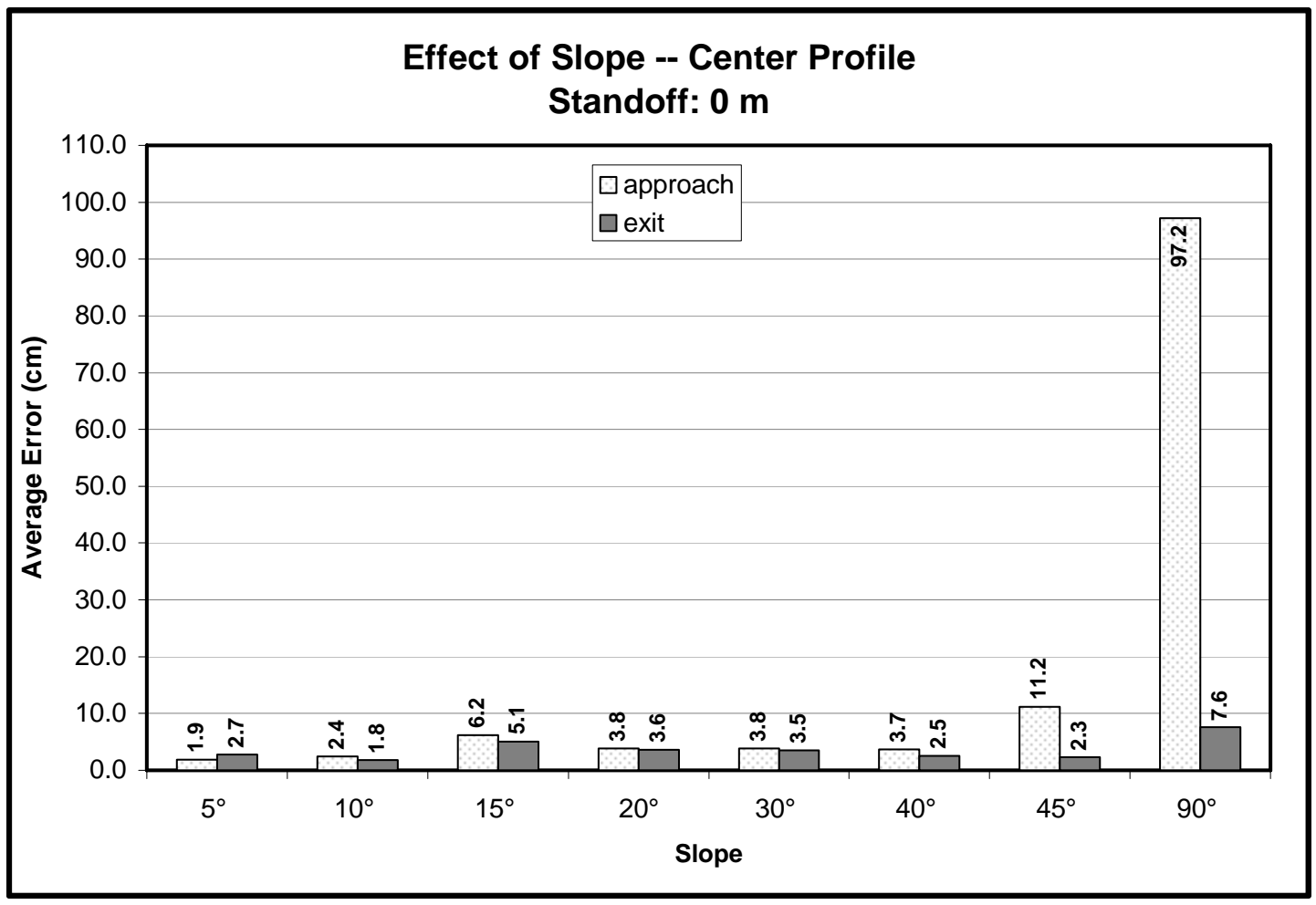

Figure 27. Average error at various slopes from a standoff of $0 \mathrm{~m}$ for the center profile 


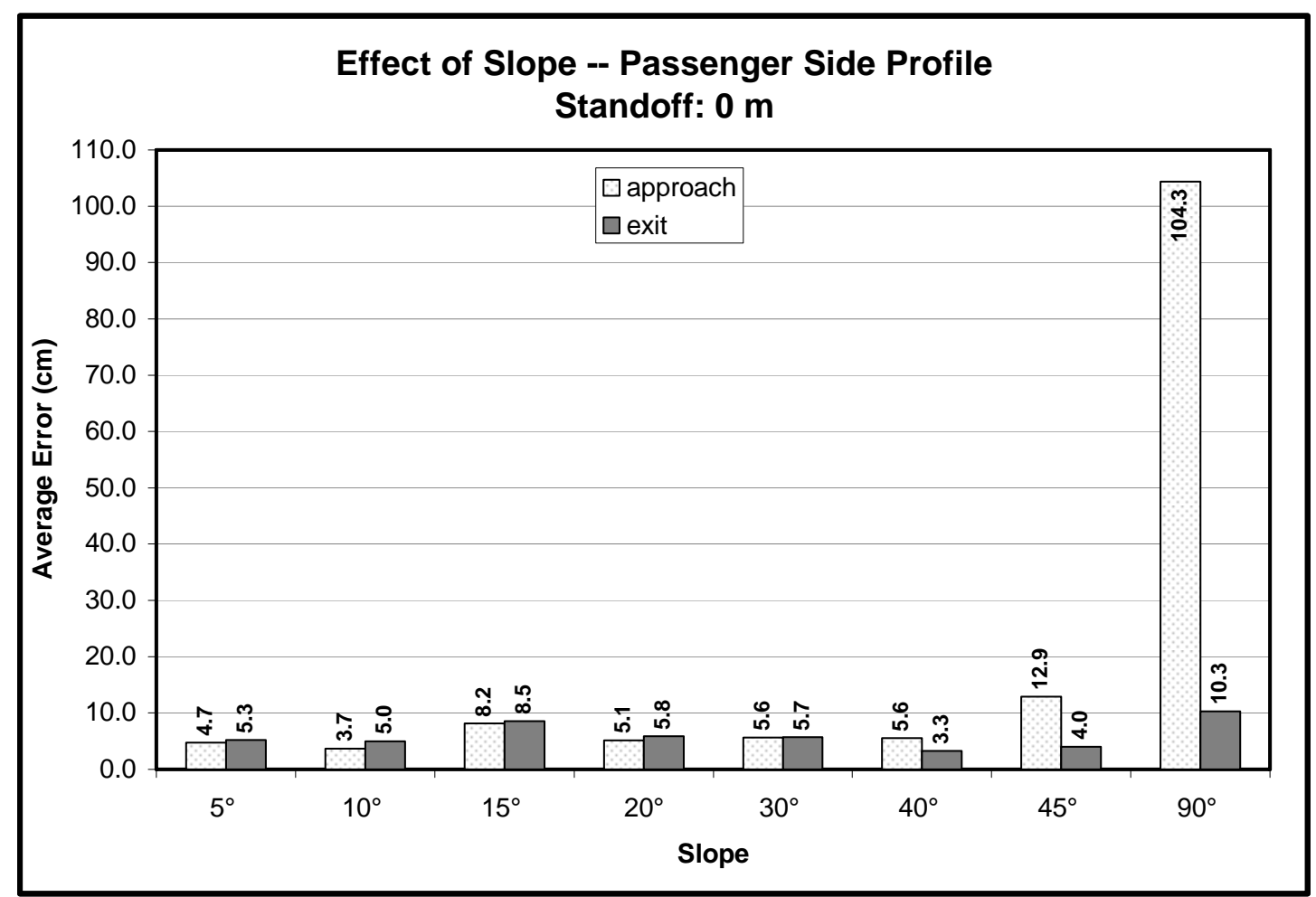

Figure 28. Average error at various slopes from a standoff of $0 \mathrm{~m}$ for the passenger side profile.

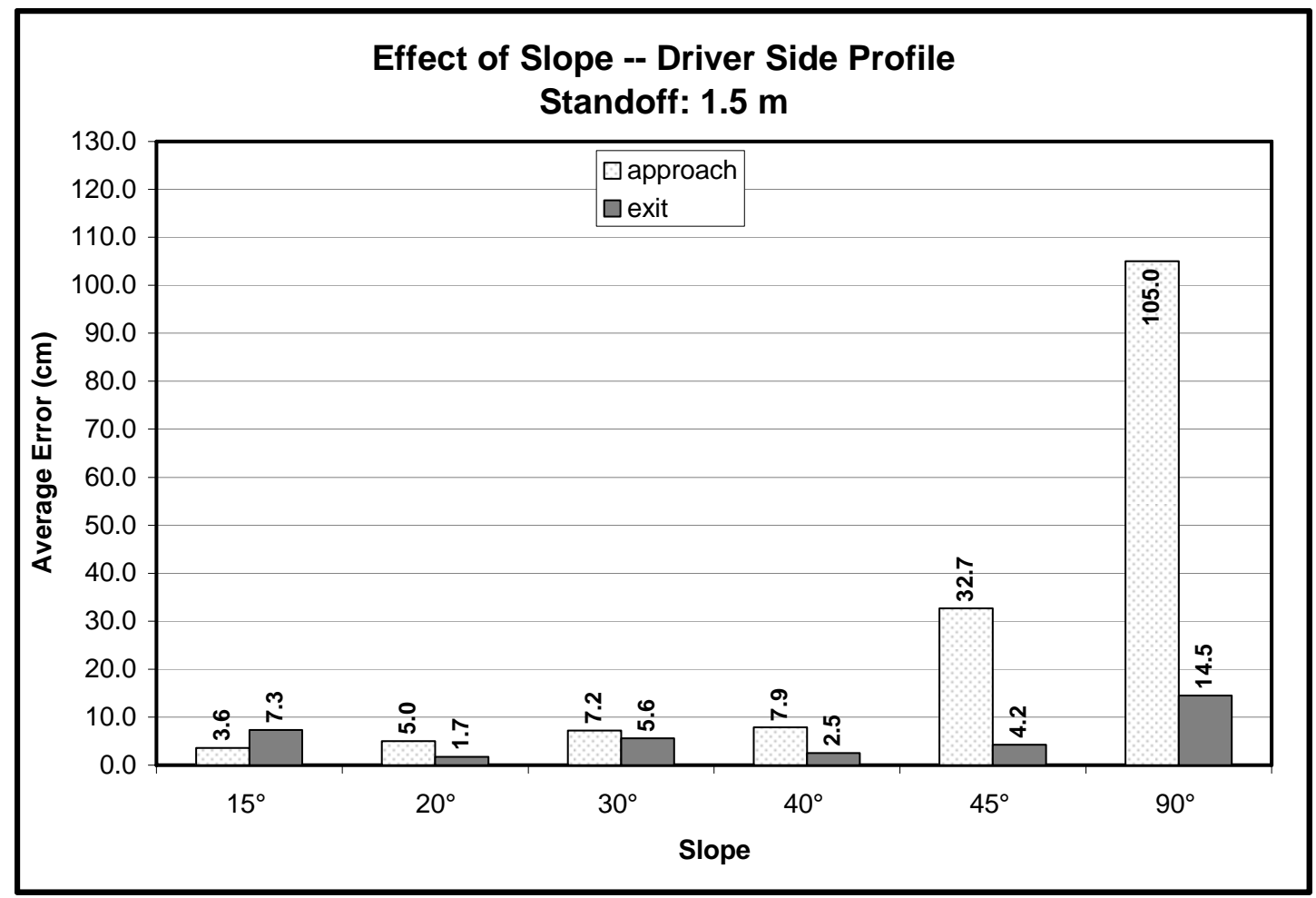

Figure 29. Average error at various slopes from a standoff of $1.5 \mathrm{~m}$ for the driver side profile. 


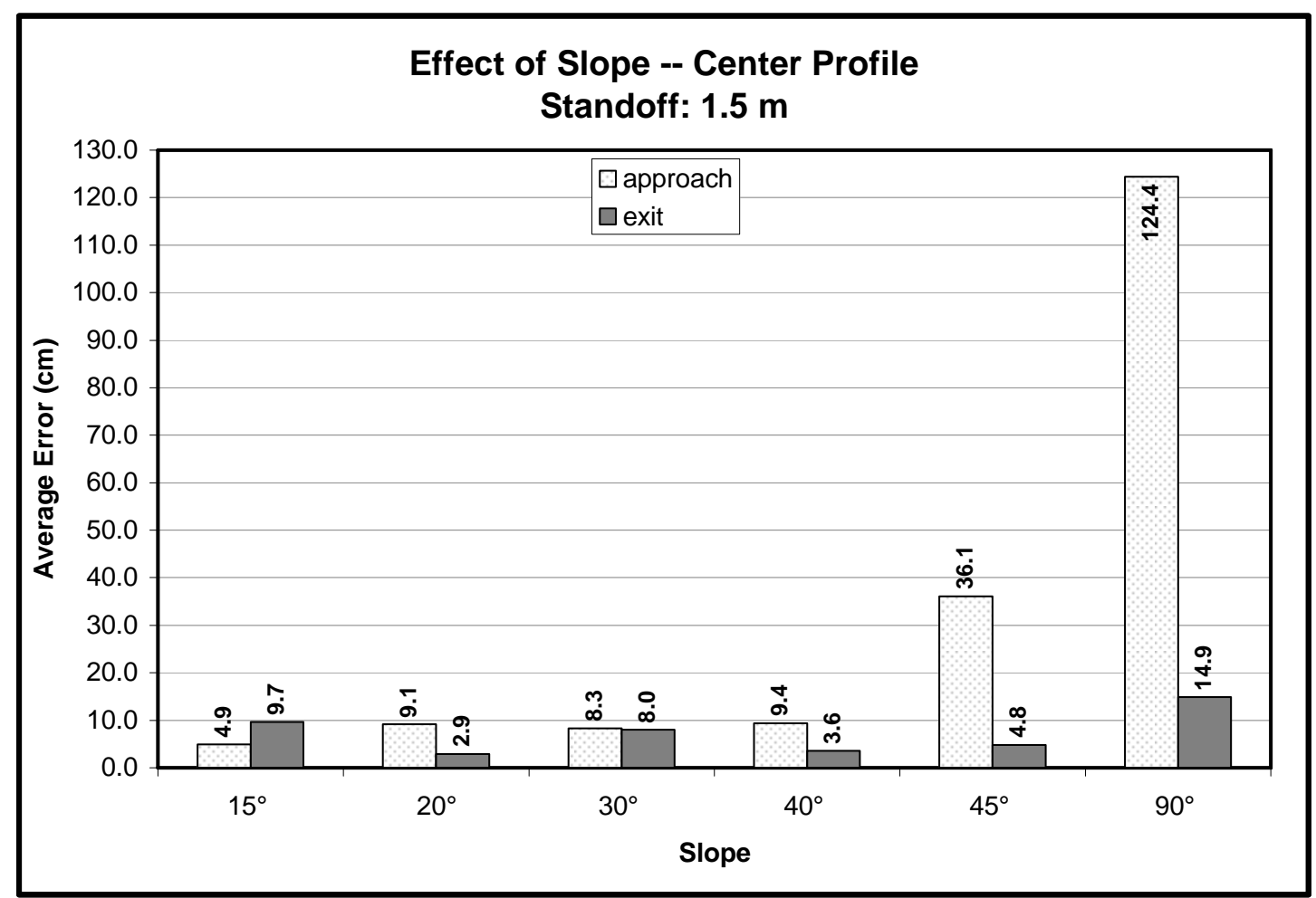

Figure 30. Average error at various slopes from a standoff of $1.5 \mathrm{~m}$ for the center profile.

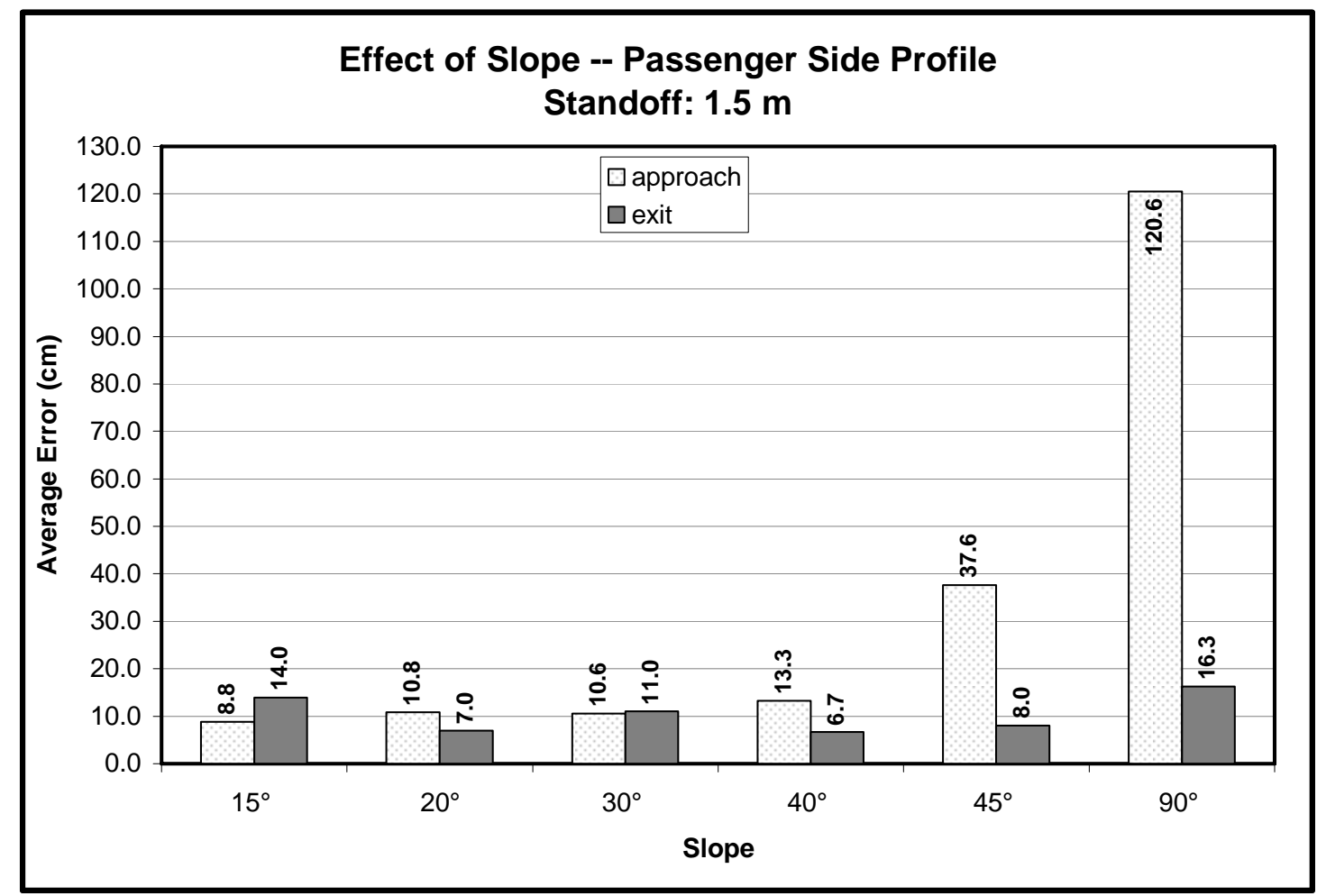

Figure 31. Average error at various slopes from a standoff of $1.5 \mathrm{~m}$ for the passenger side profile. 


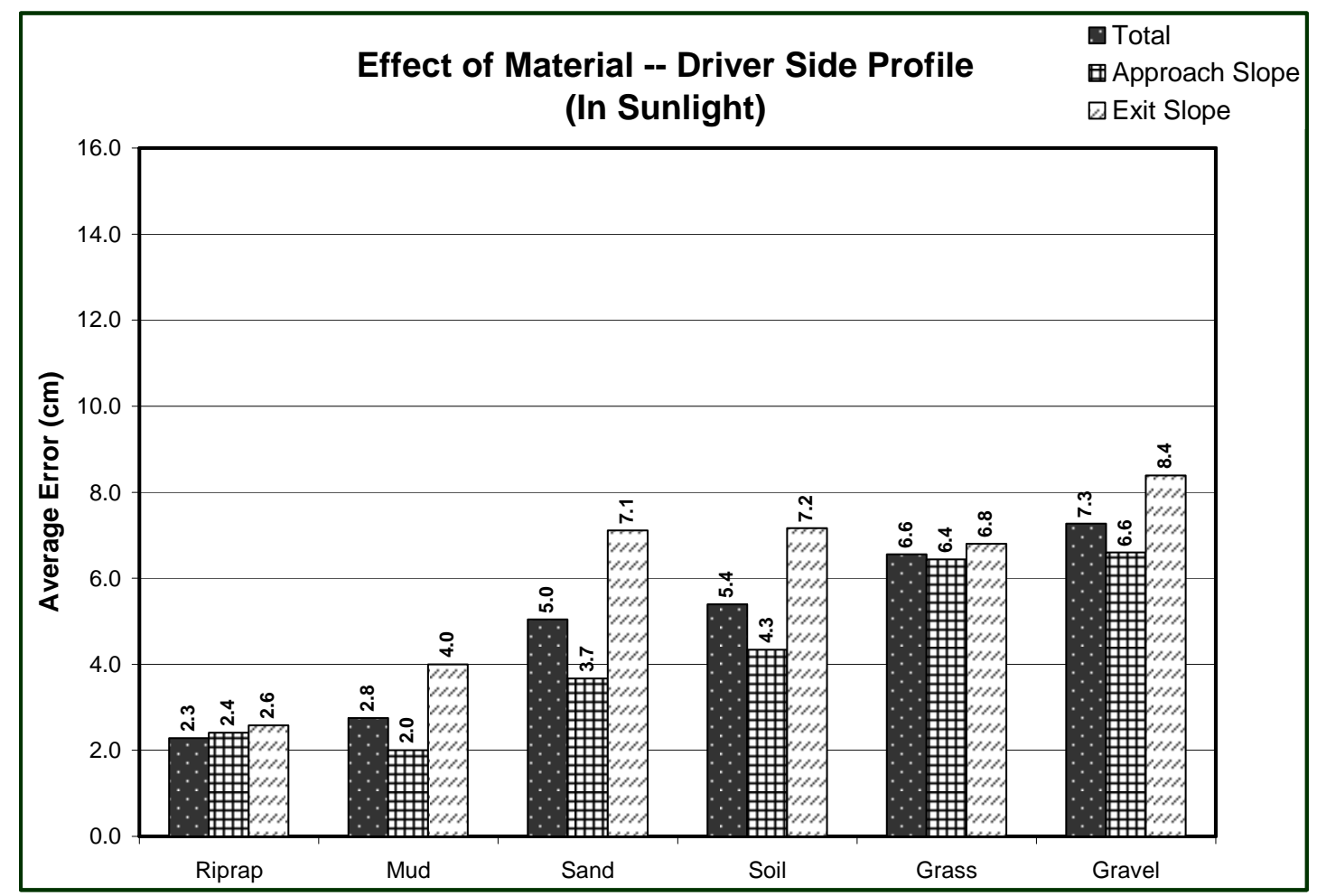

Figure 32. Effect of surface materials in sunlight for the driver side profile.

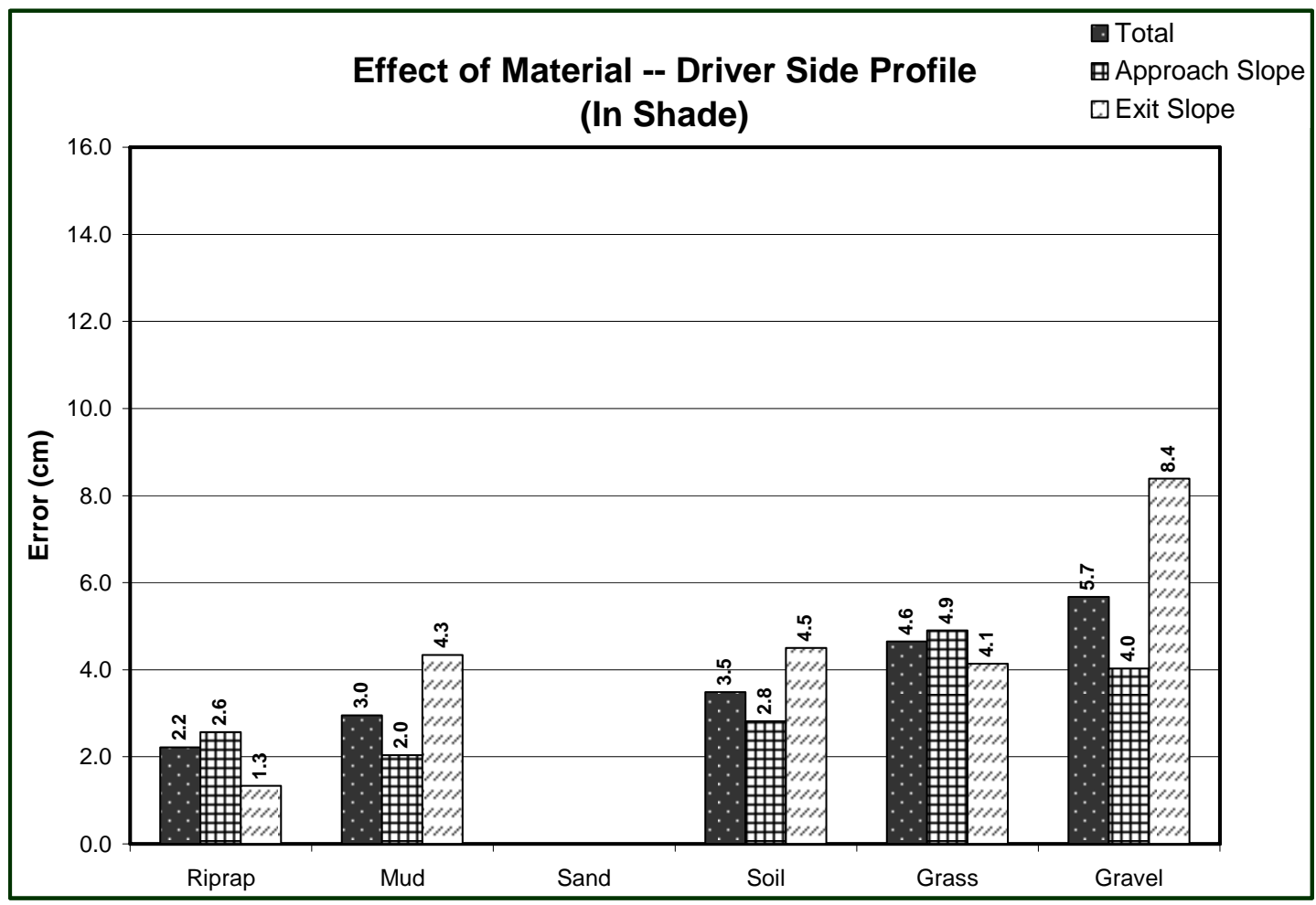

Figure 33. Effect of surface materials in shade for the driver side profile. 


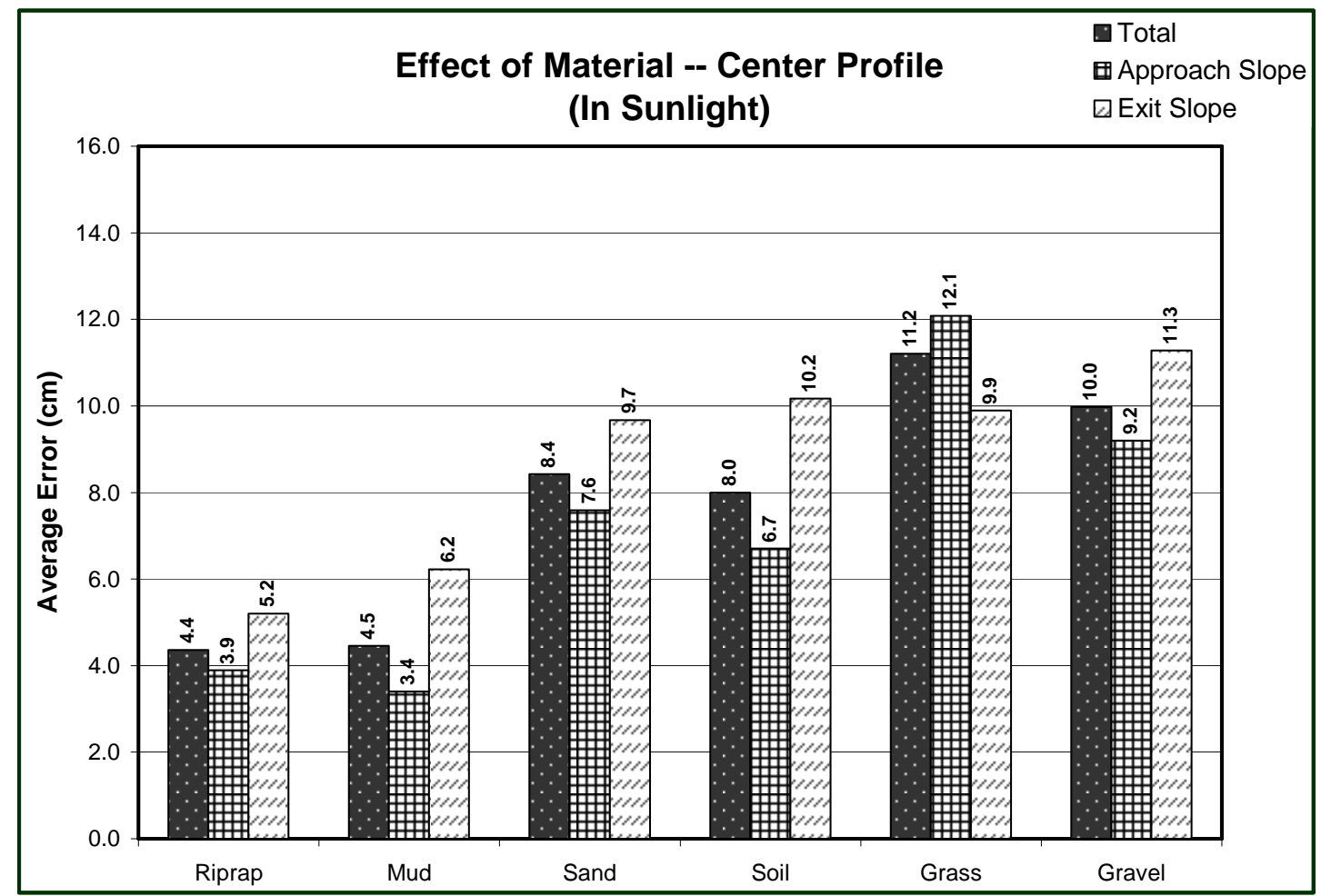

Figure 34. Effect of surface materials in sunlight for the center profile.

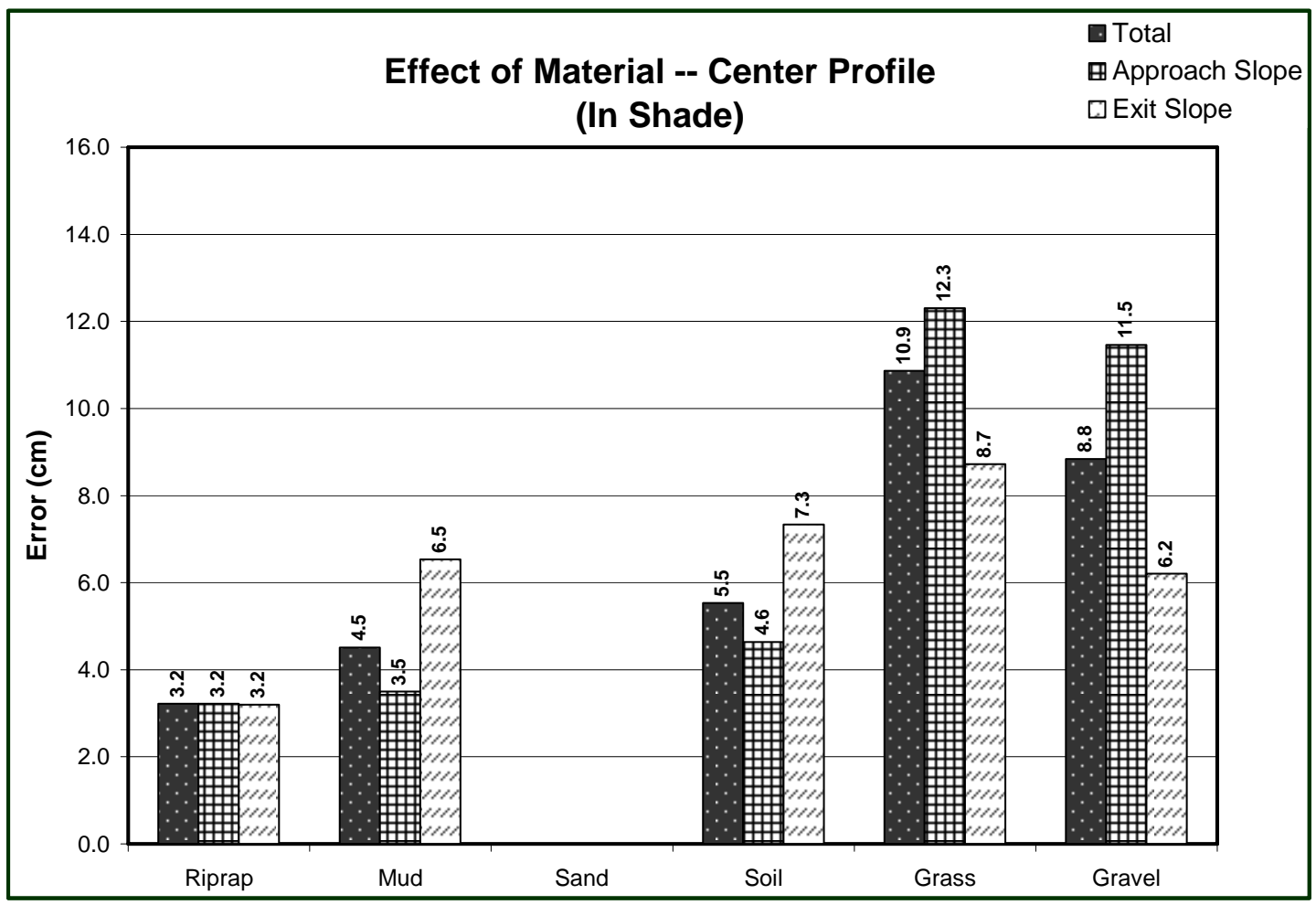

Figure 35. Effect of surface materials in shade for the center profile. 


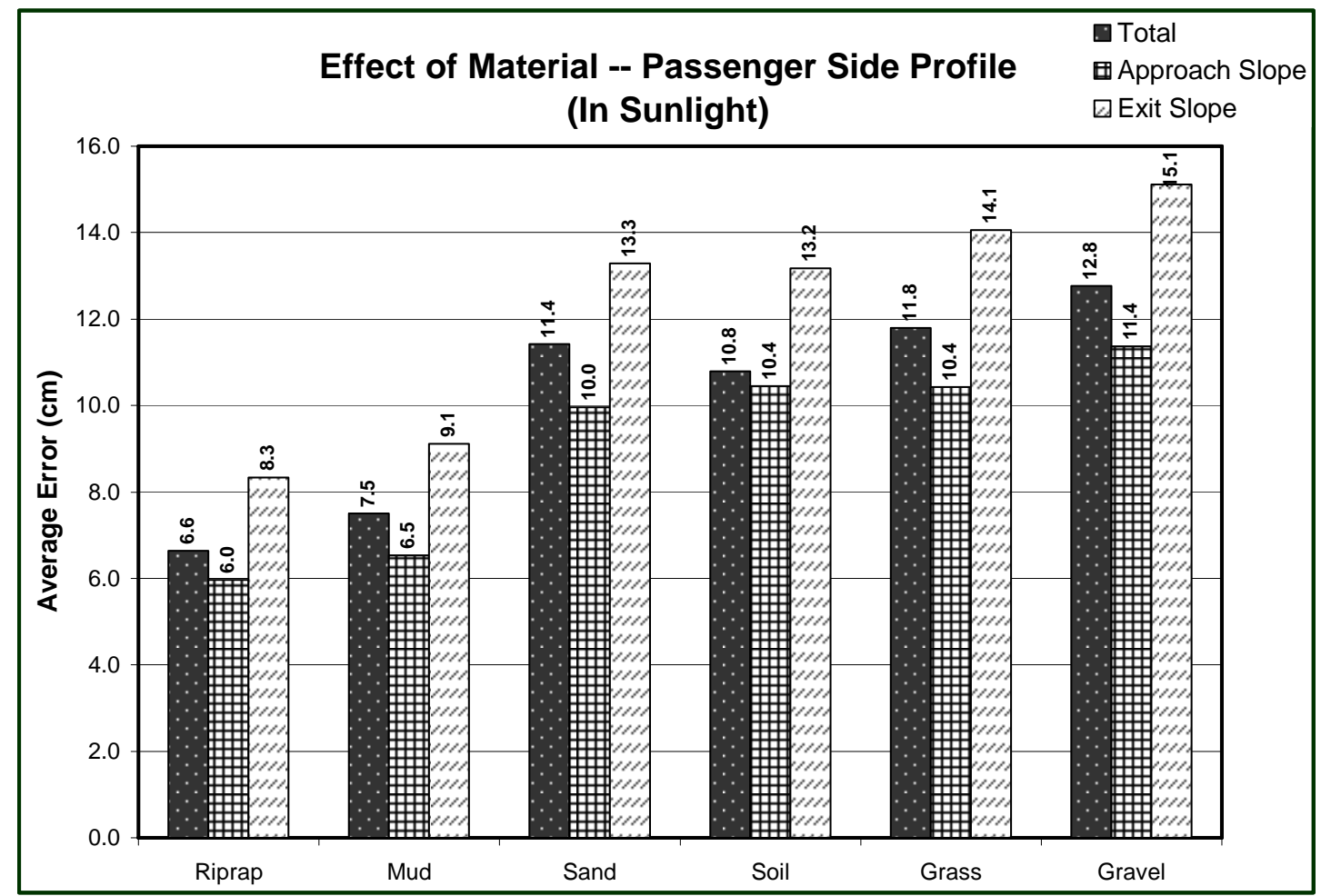

Figure 36. Effect of surface materials in sunlight for the passenger side profile.

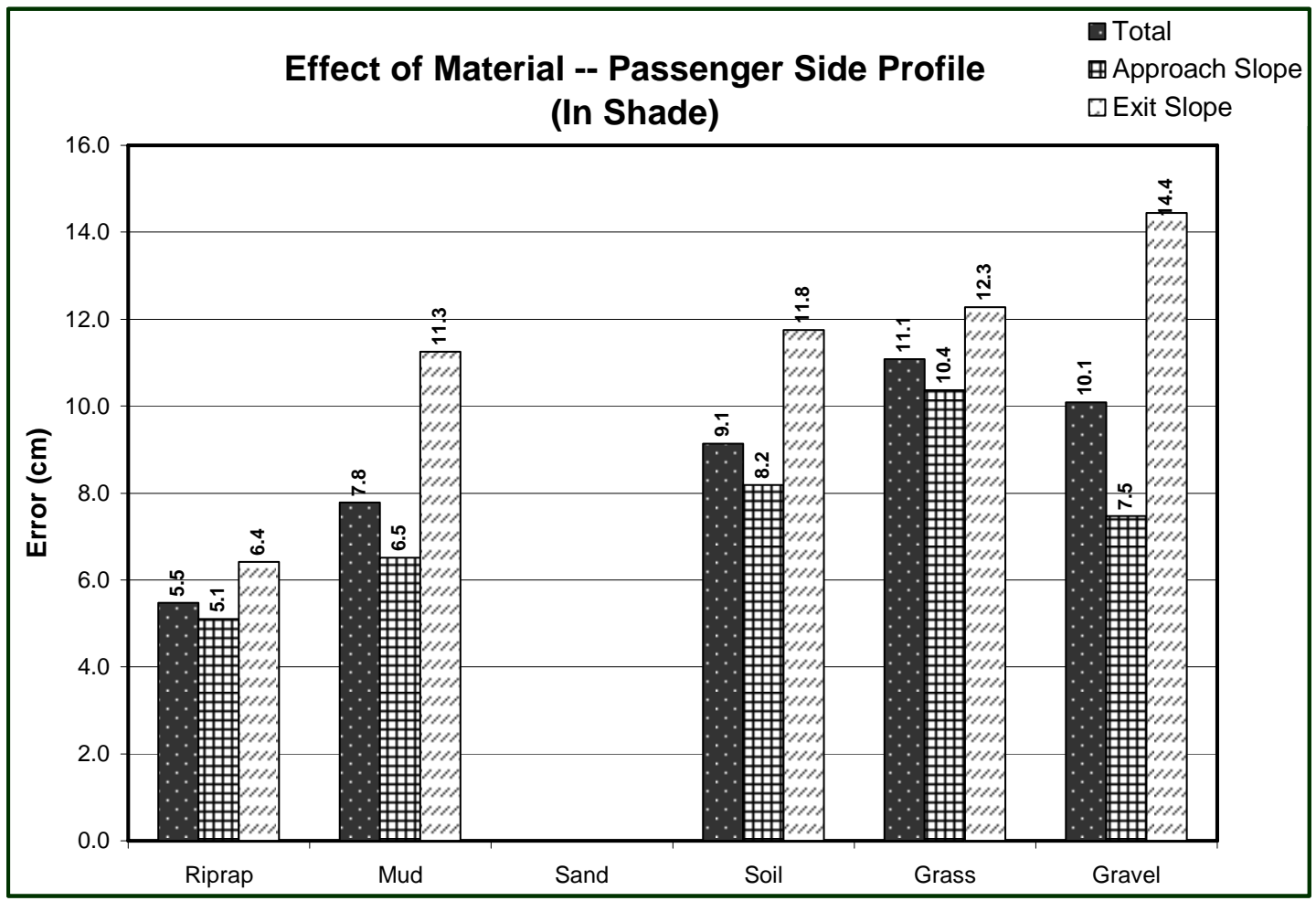

Figure 37. Effect of surface materials in shade for the passenger side profile. 


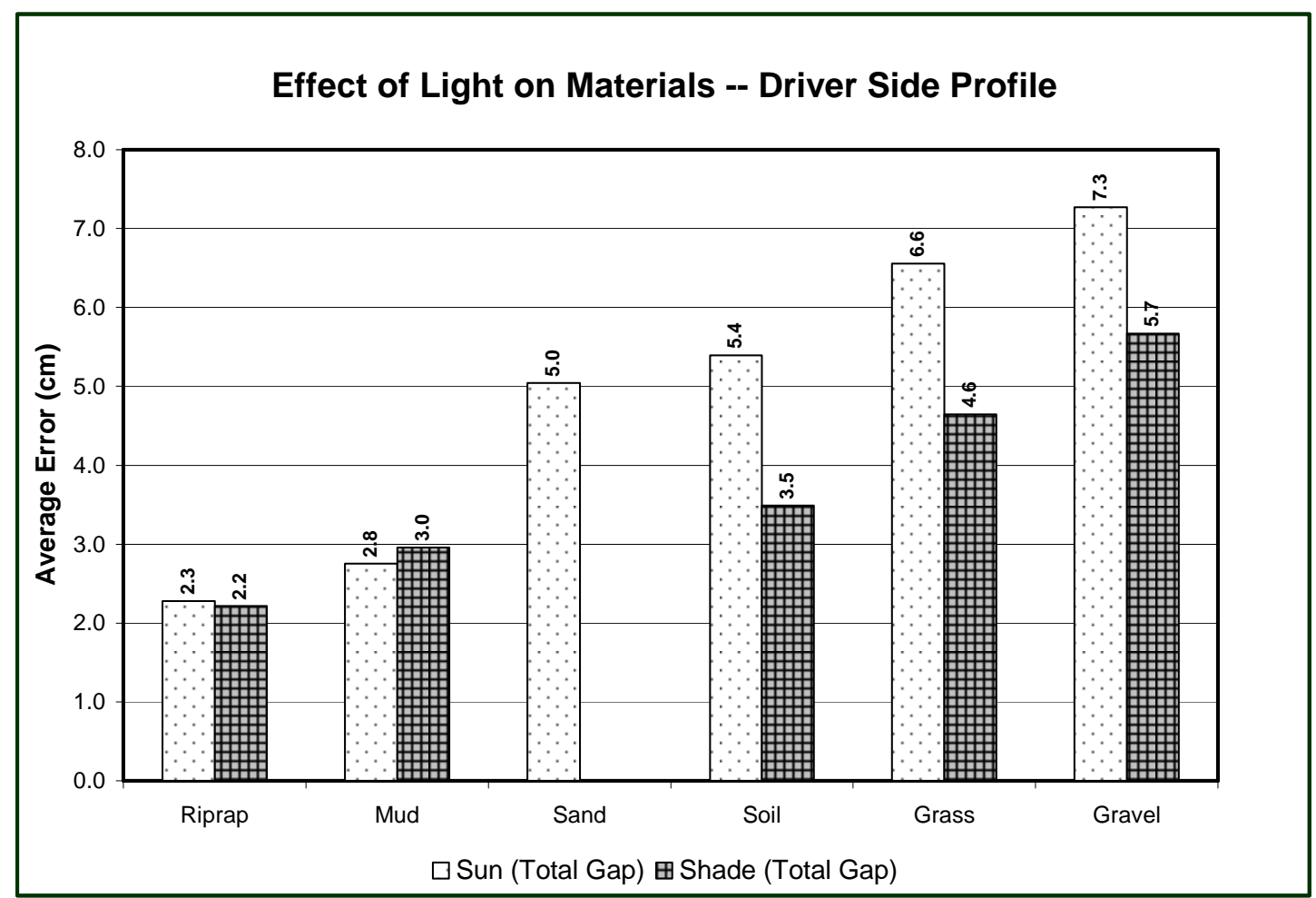

Figure 38. Effect of collecting data in sun or shade at material gap for the driver side profile.

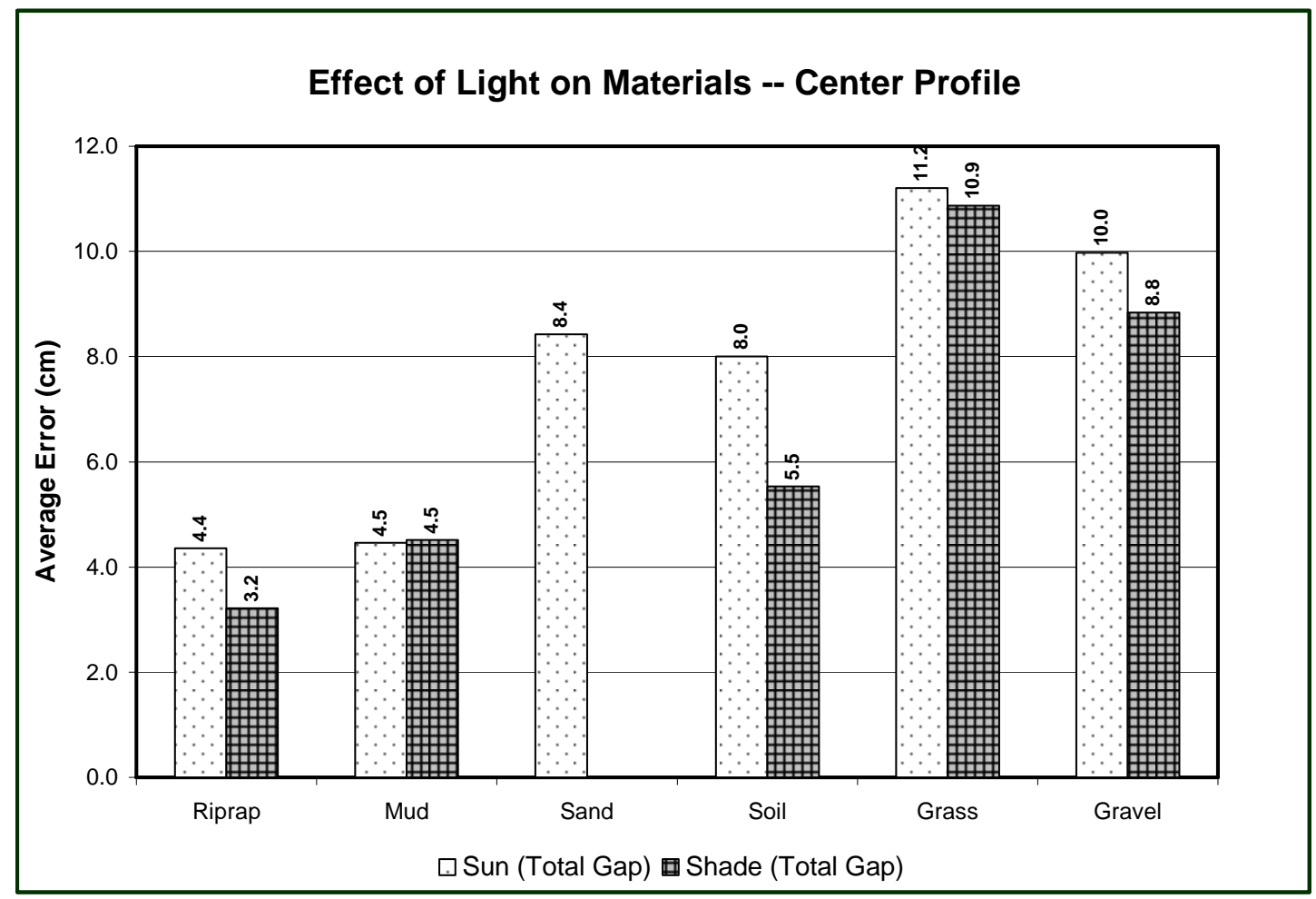

Figure 39. Effect of collecting data in sun or shade at material gap for the center profile. 


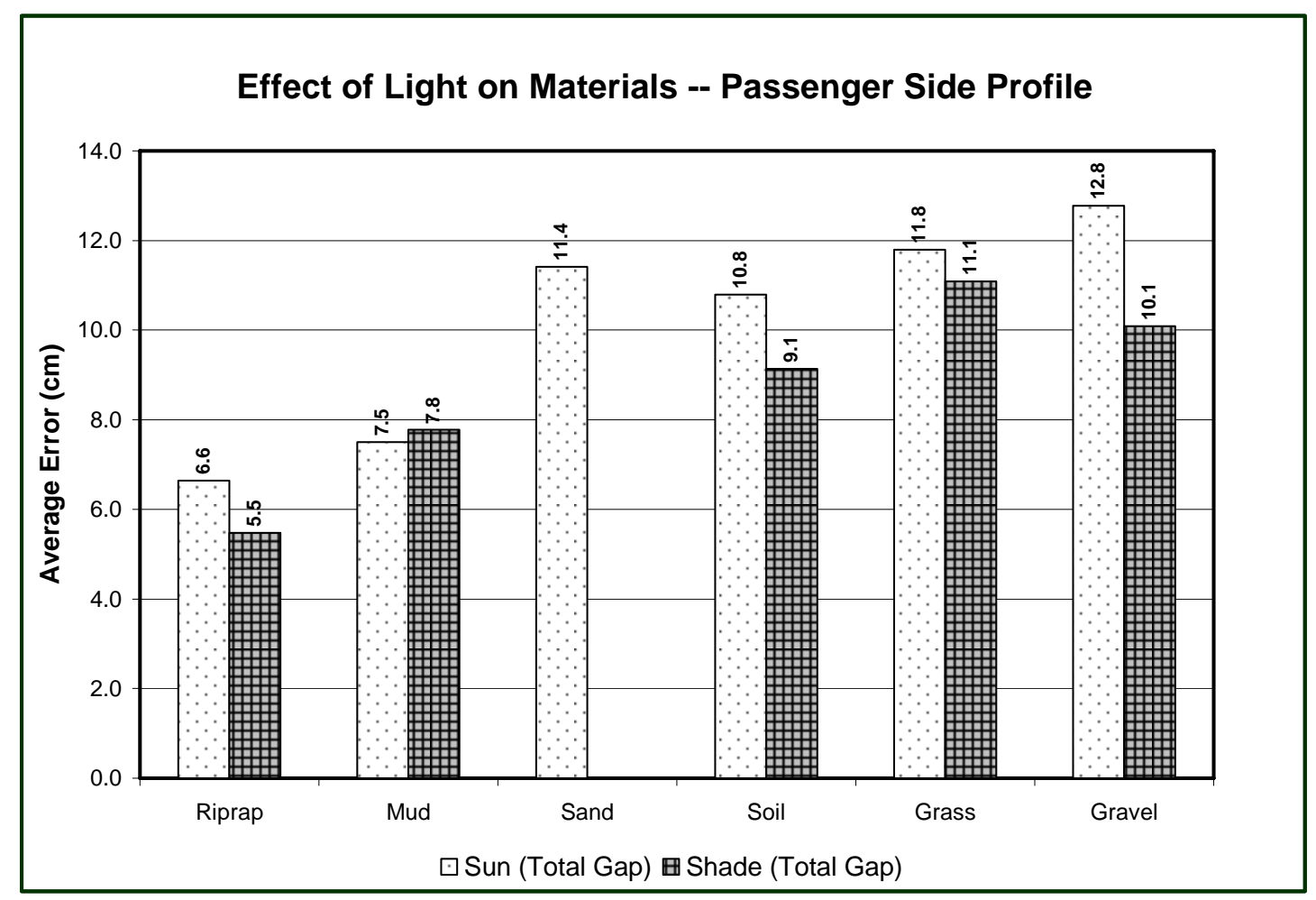

Figure 40. Effect of collecting data in sun or shade at material gap for the passenger side profile.

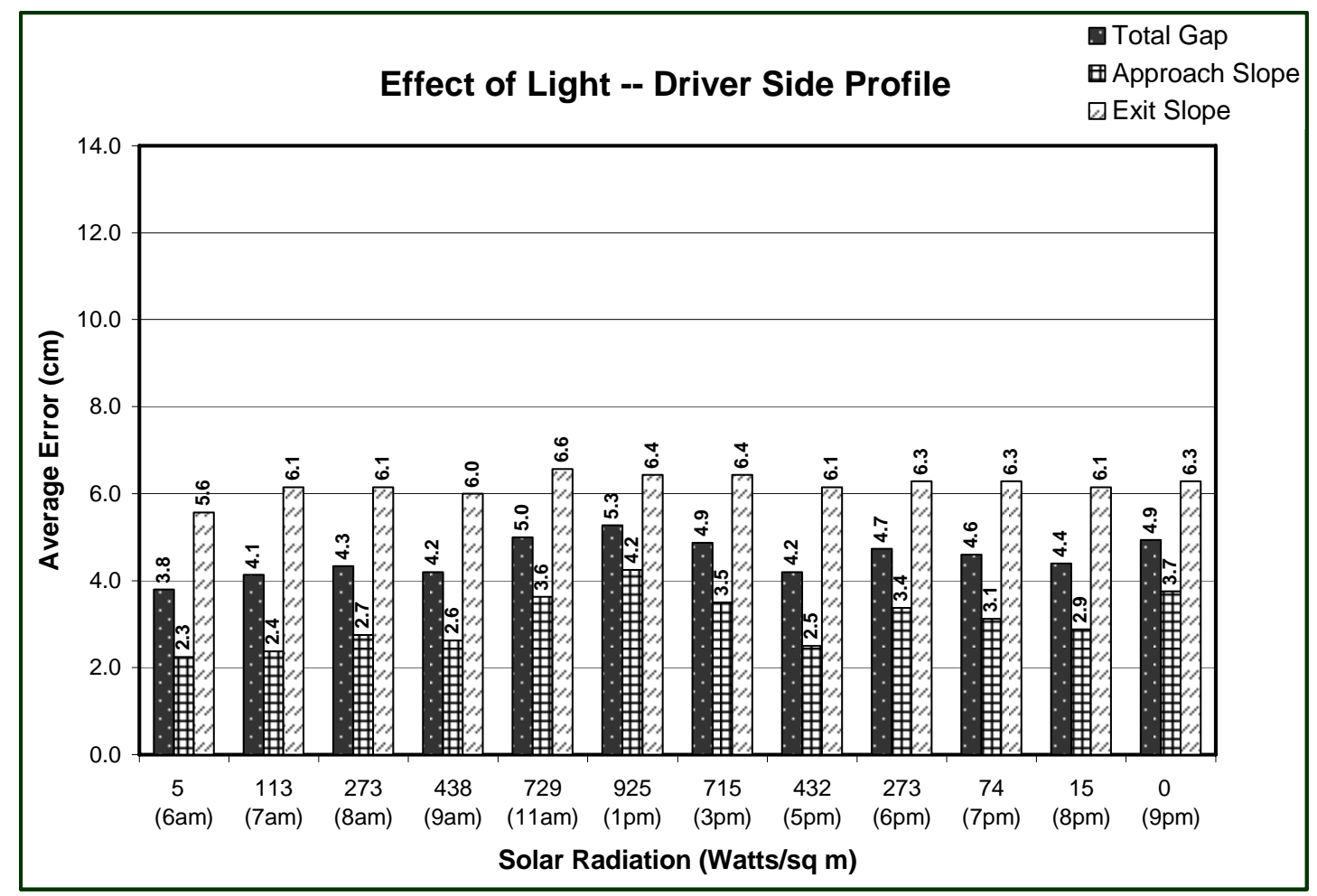

Figure 41. Effect of ambient lighting for the driver side profile. 


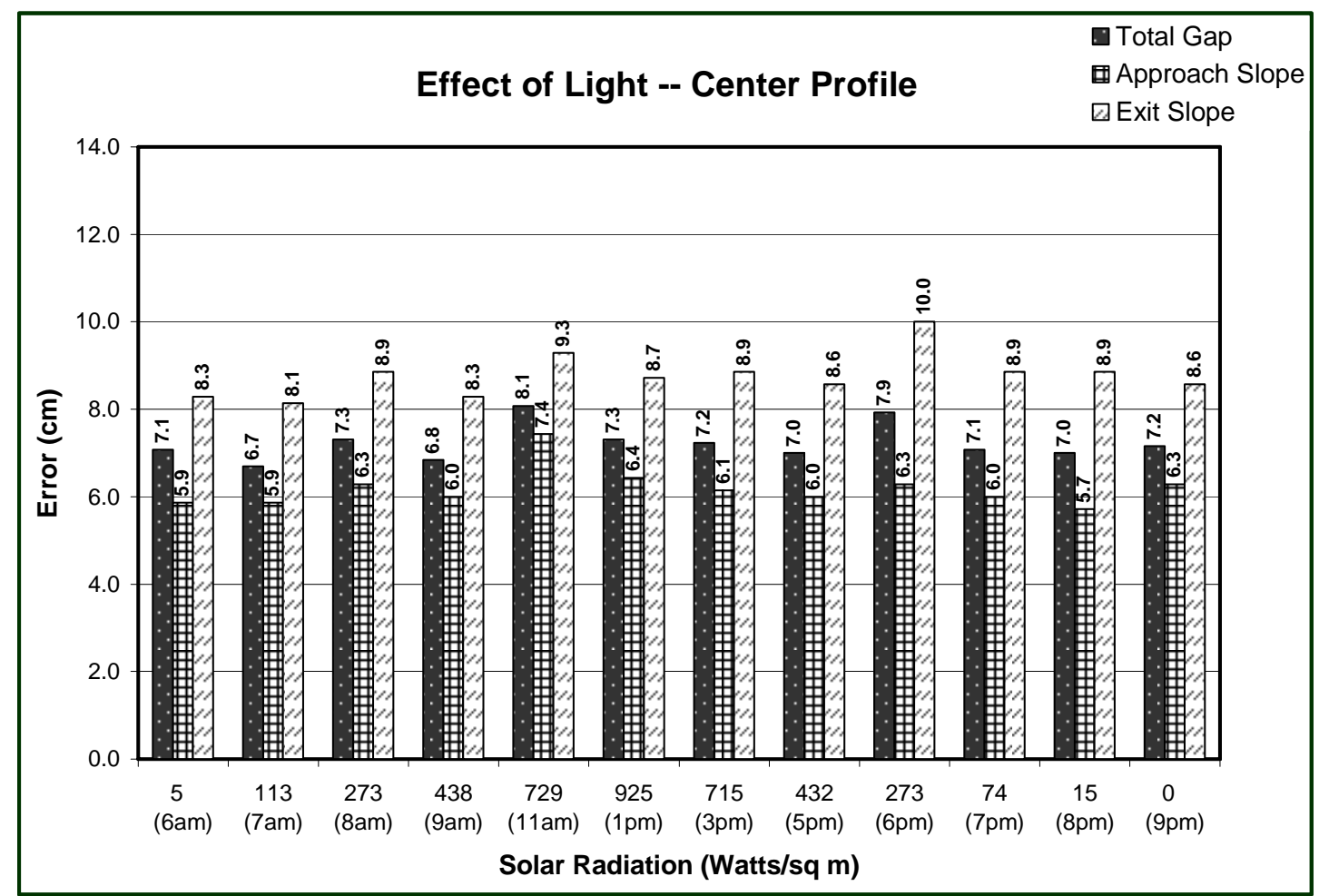

Figure 42. Effect of ambient lighting for the center profile.

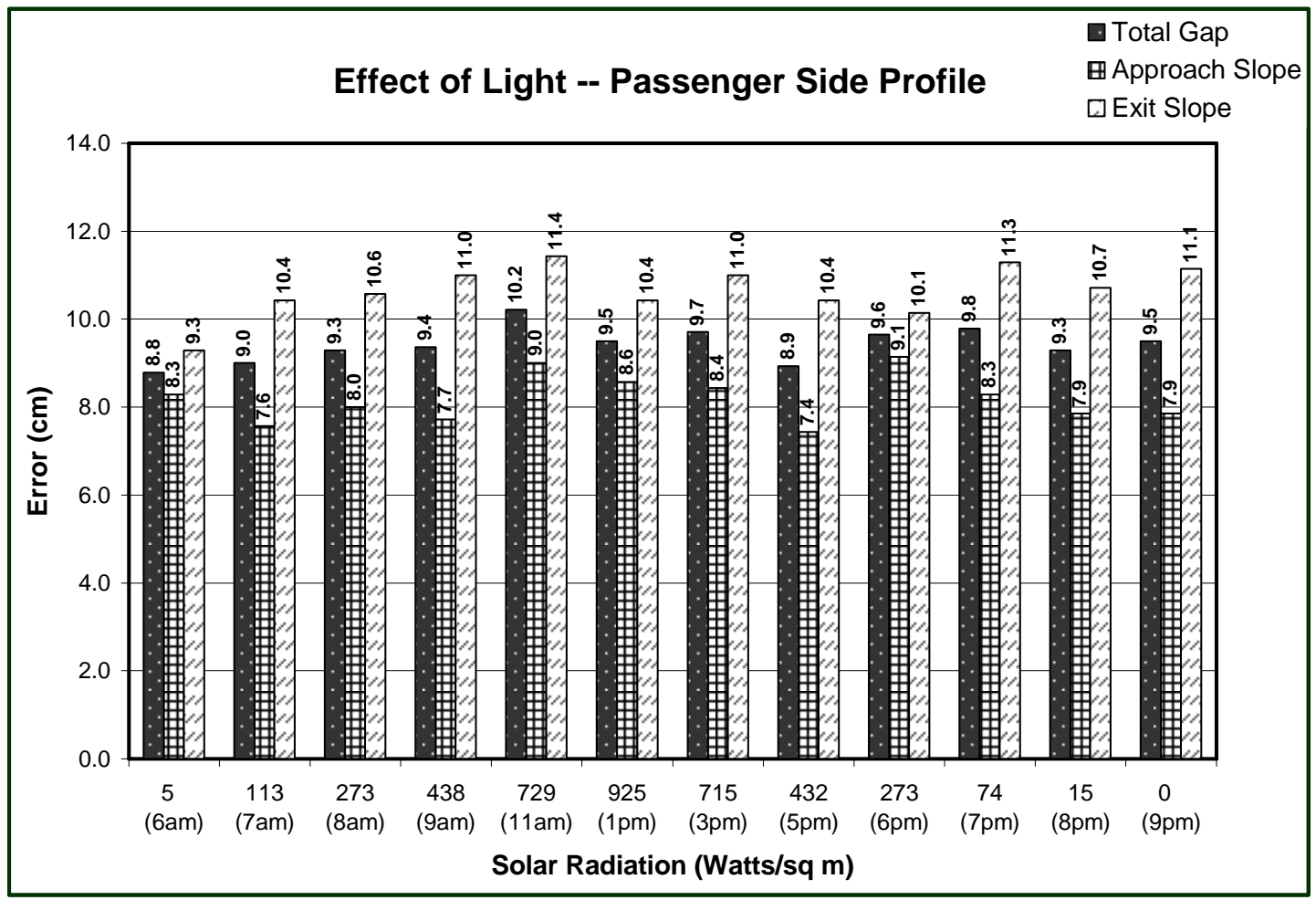

Figure 43. Effect of ambient lighting for the passenger side profile. 


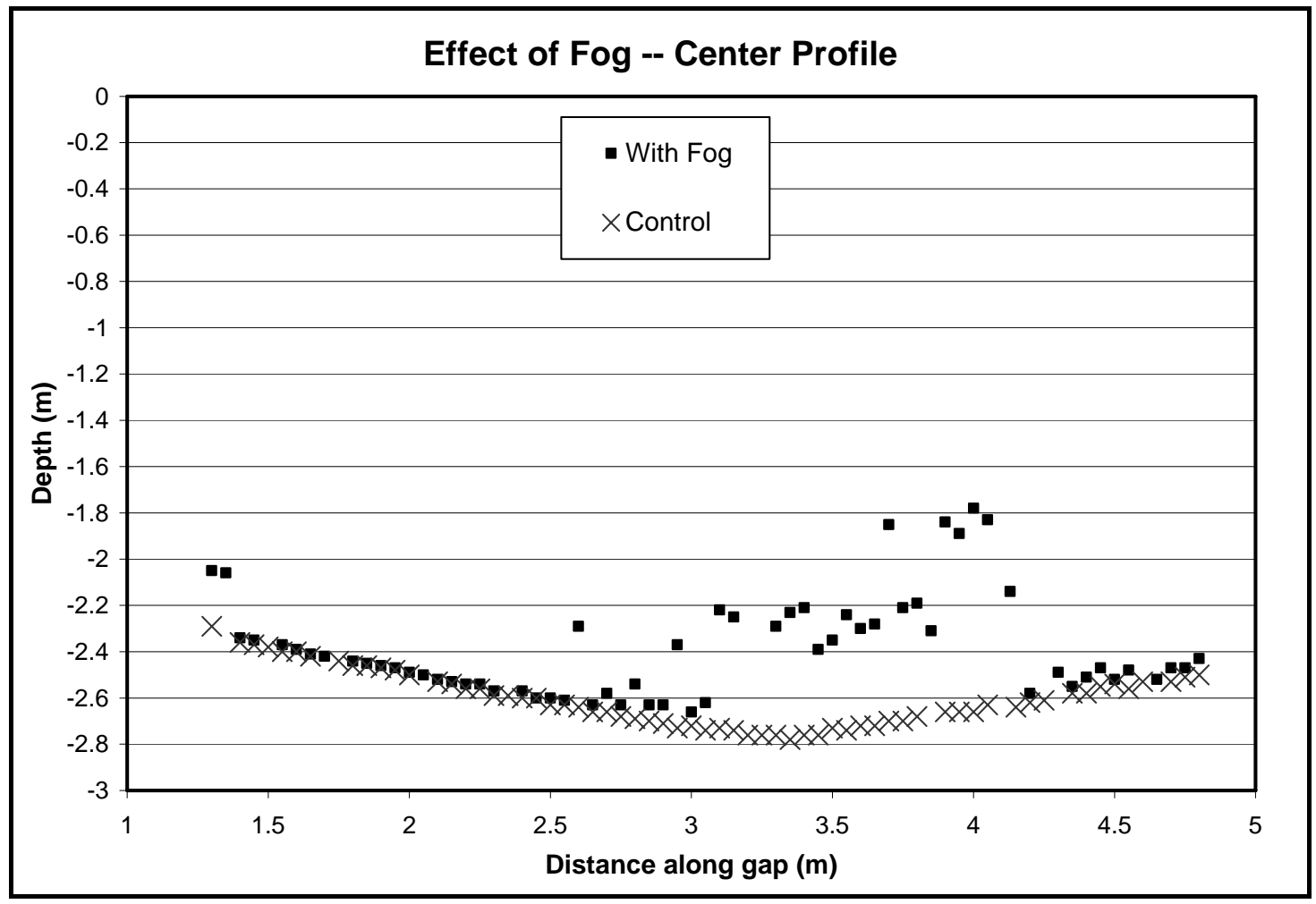

Figure 44. Effect of fog on soil gap for the center profile.

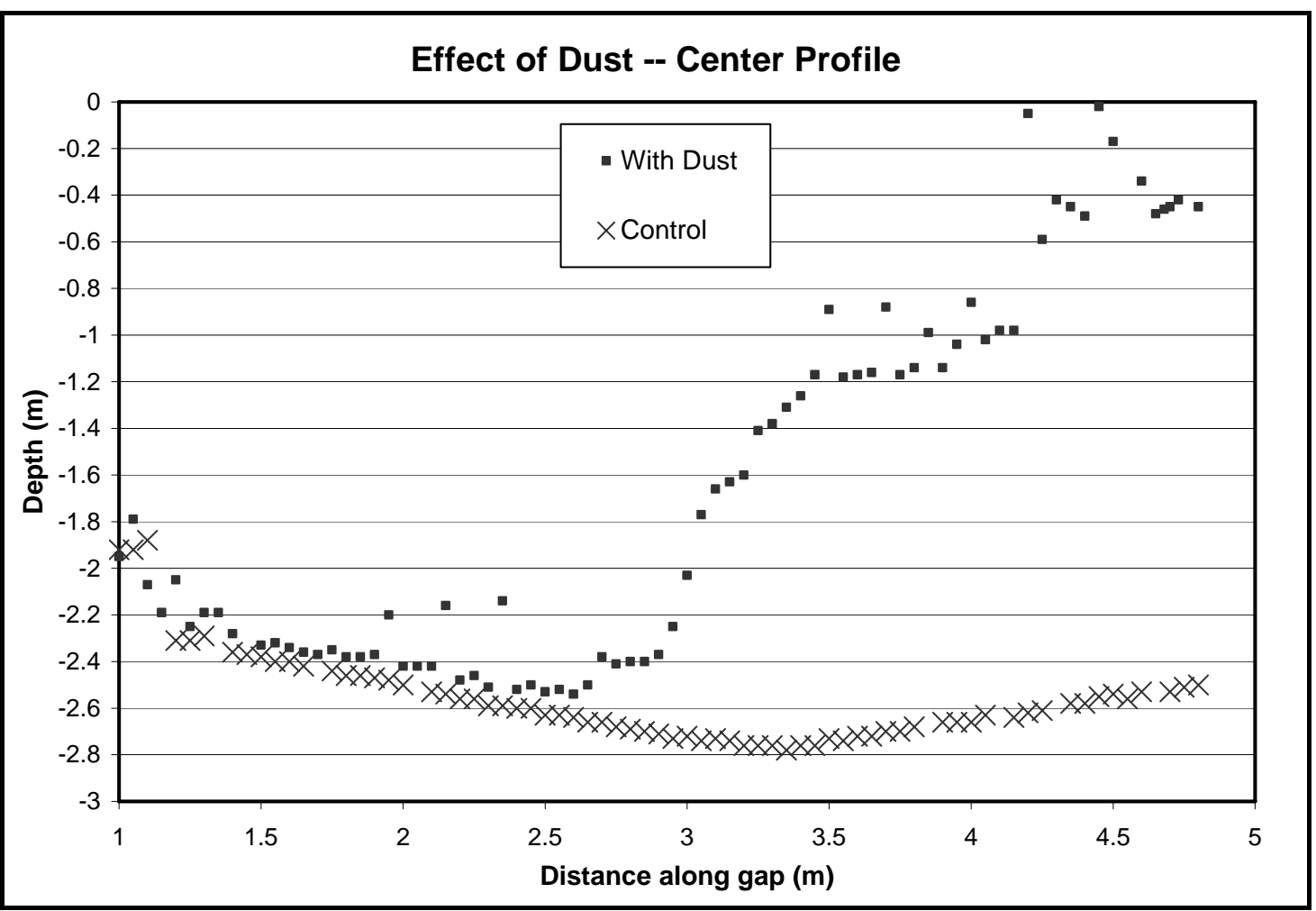

Figure 45. Effect of dust on soil gap for the center profile. 


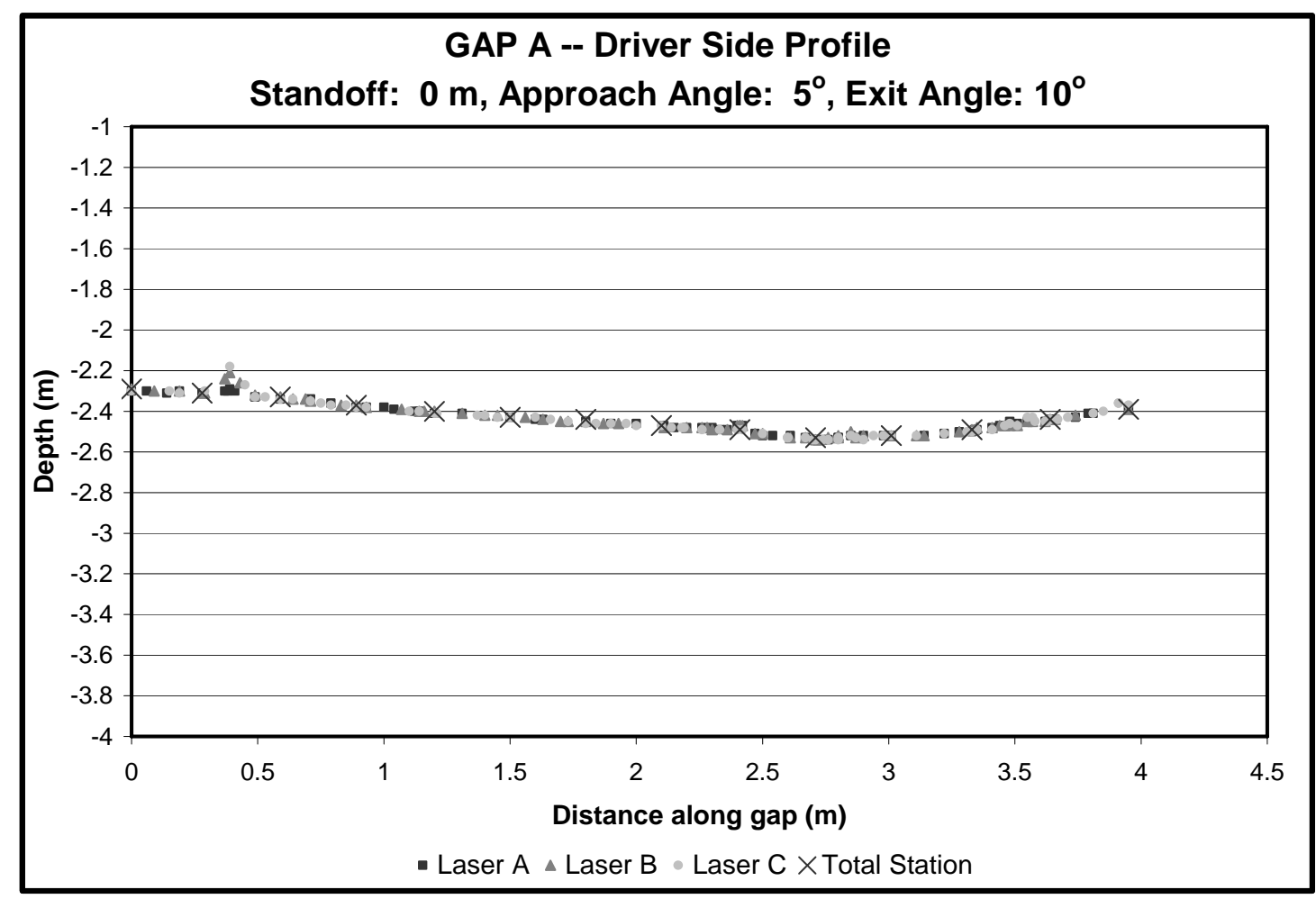

Figure 46. Data for Gap A - driver side profile.

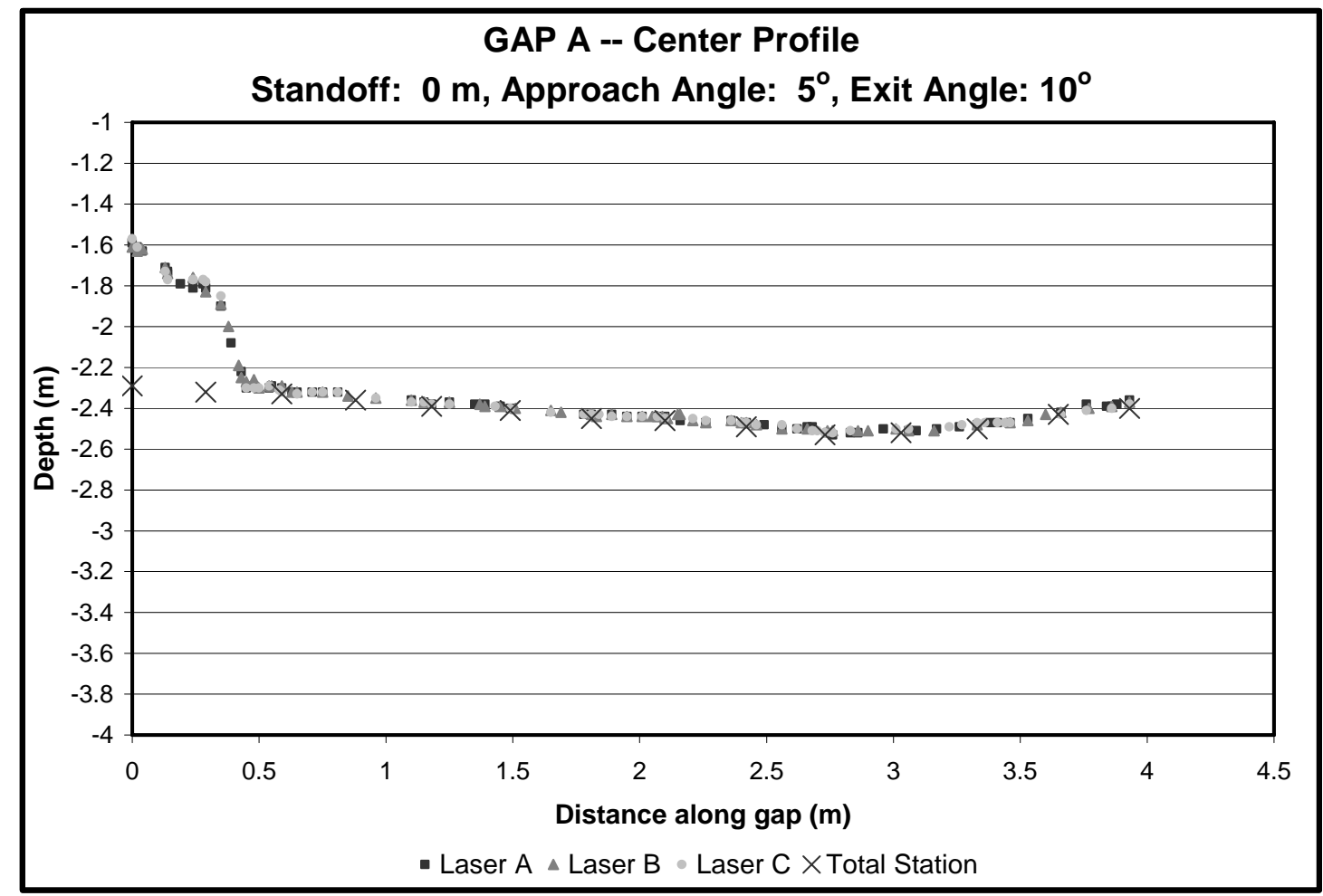

Figure 47. Data for Gap A - center profile. 


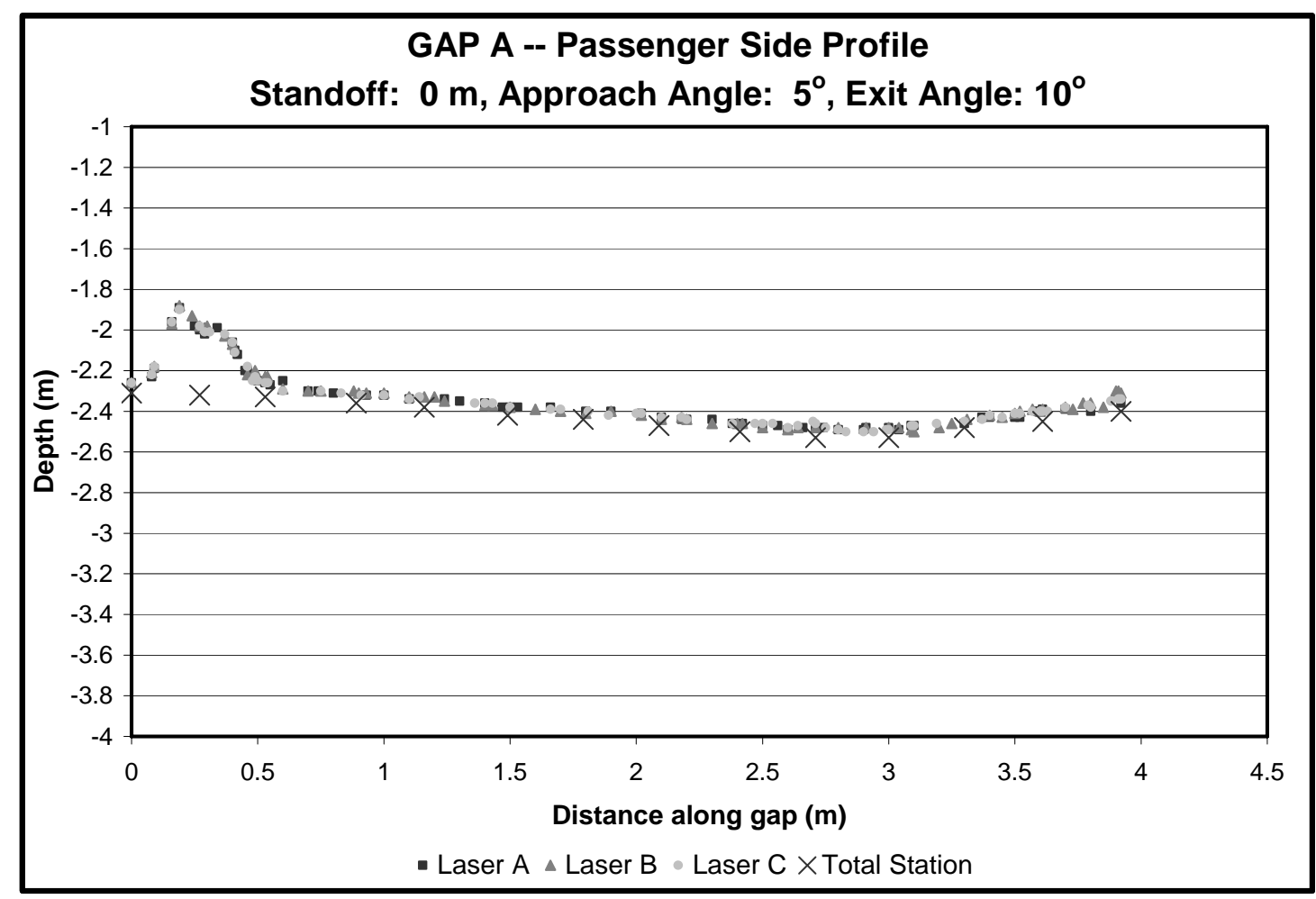

Figure 48. Data for Gap A - passenger side profile.

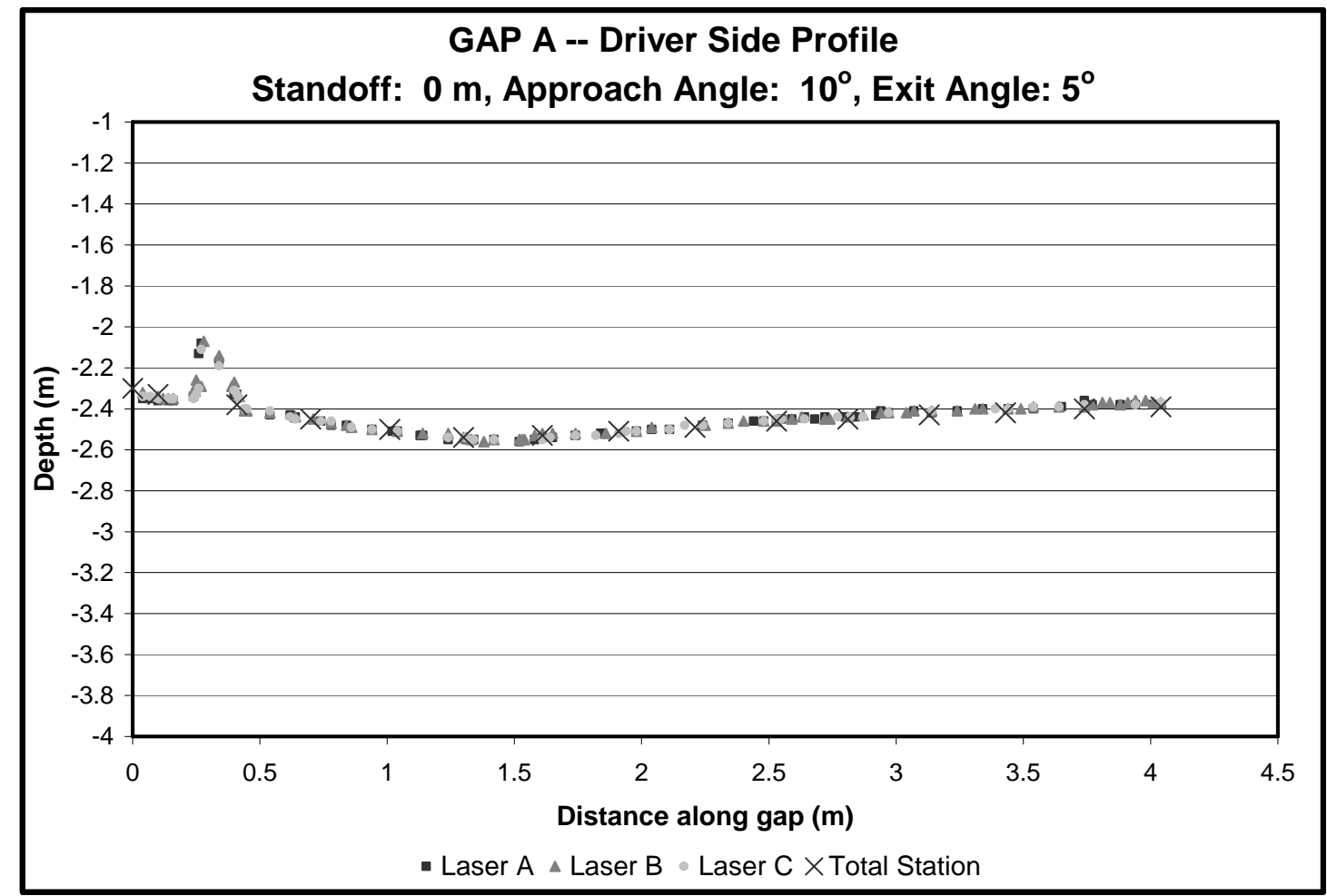

Figure 49. Data for Gap A - driver side profile. 


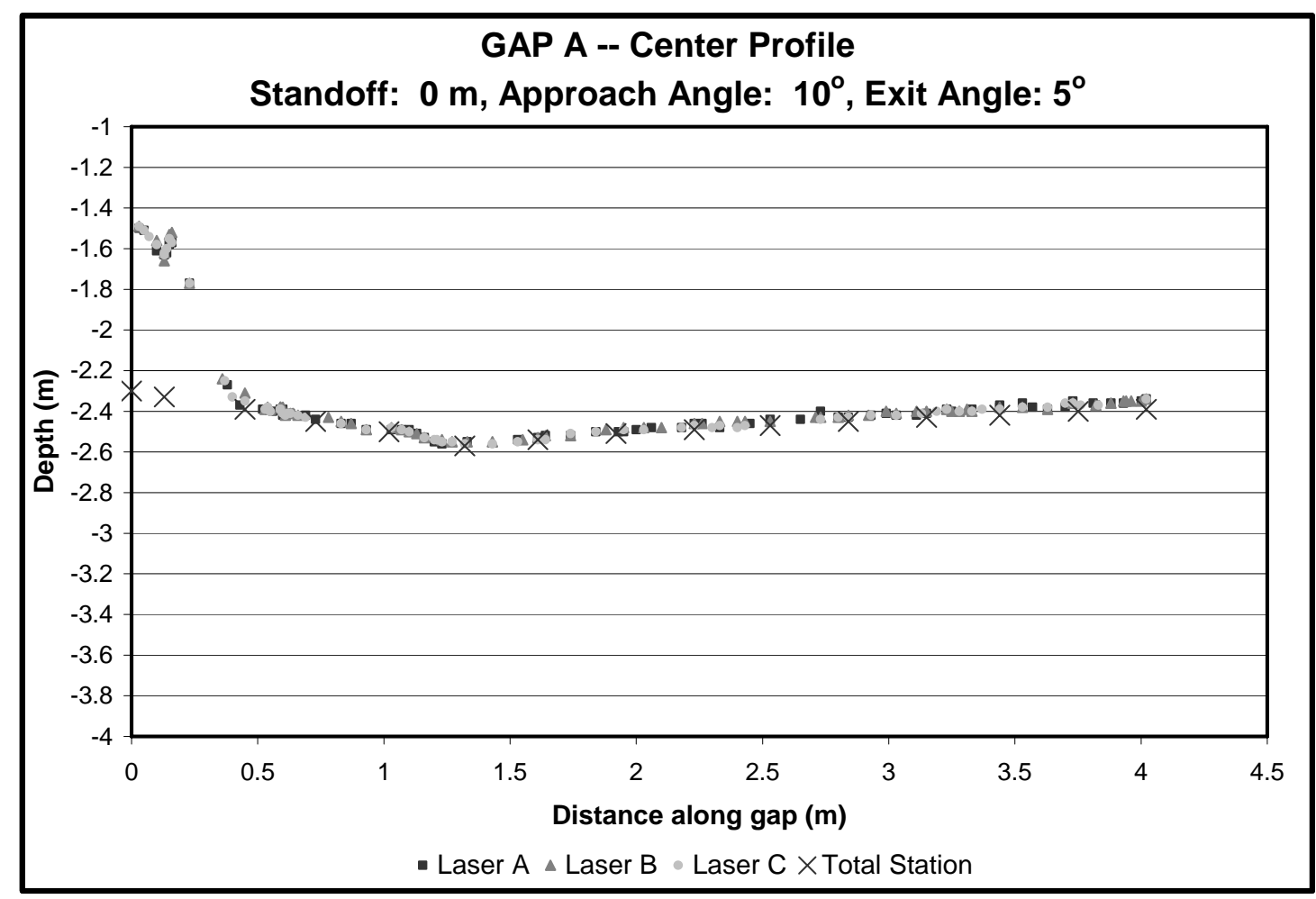

Figure 50. Data for Gap A - center profile.

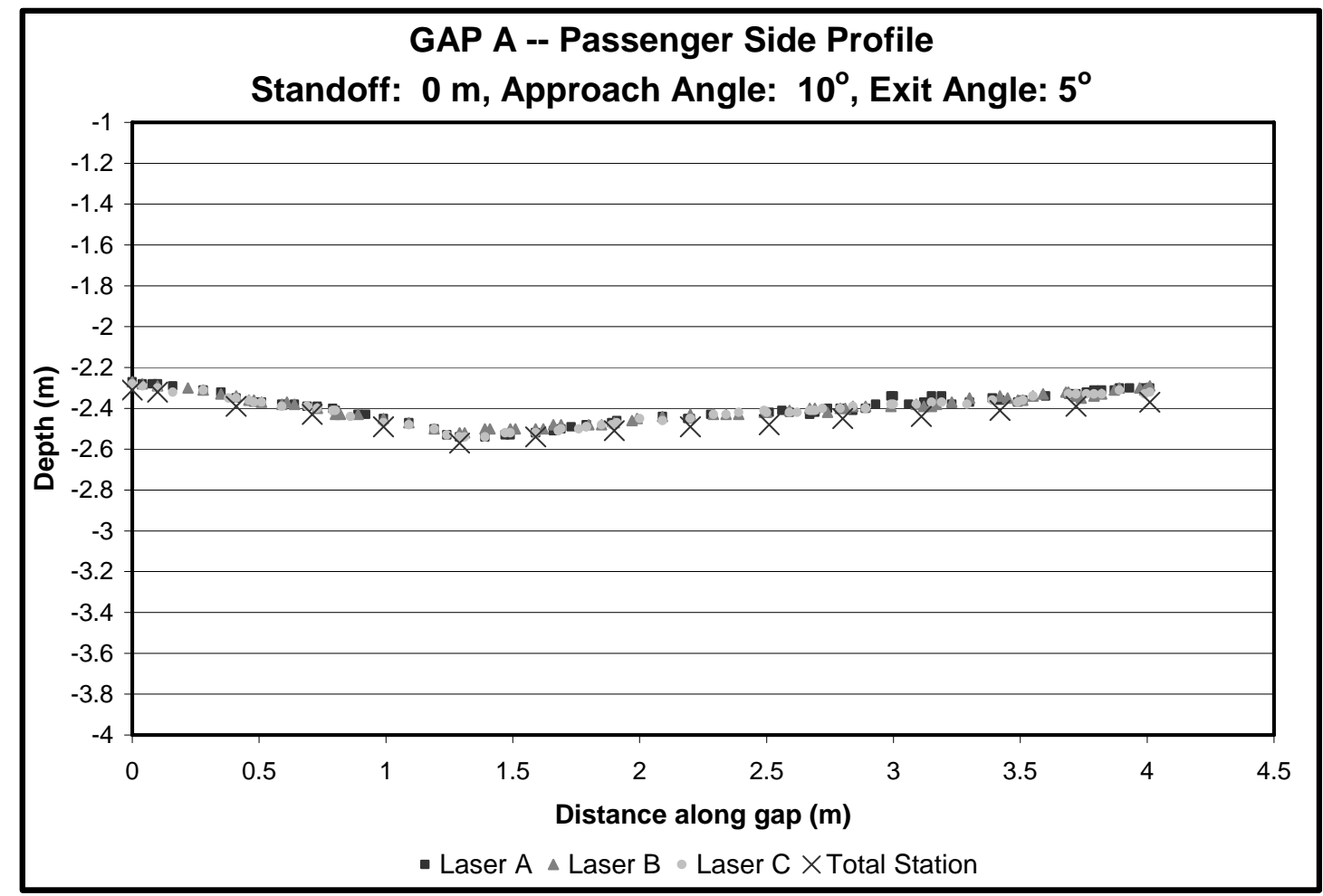

Figure 51. Data for Gap A - passenger side profile. 


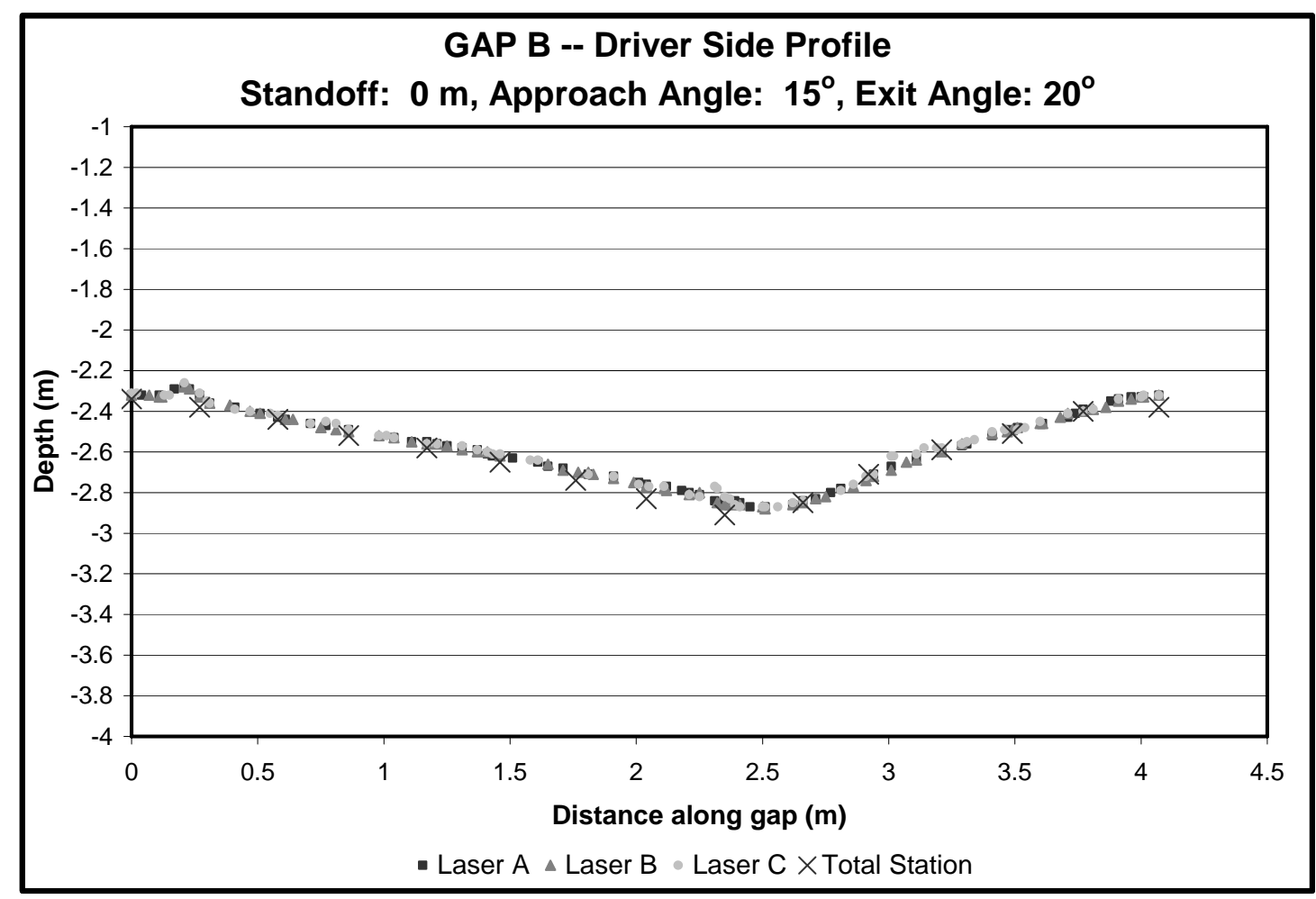

Figure 52. Data for Gap B - driver side profile.

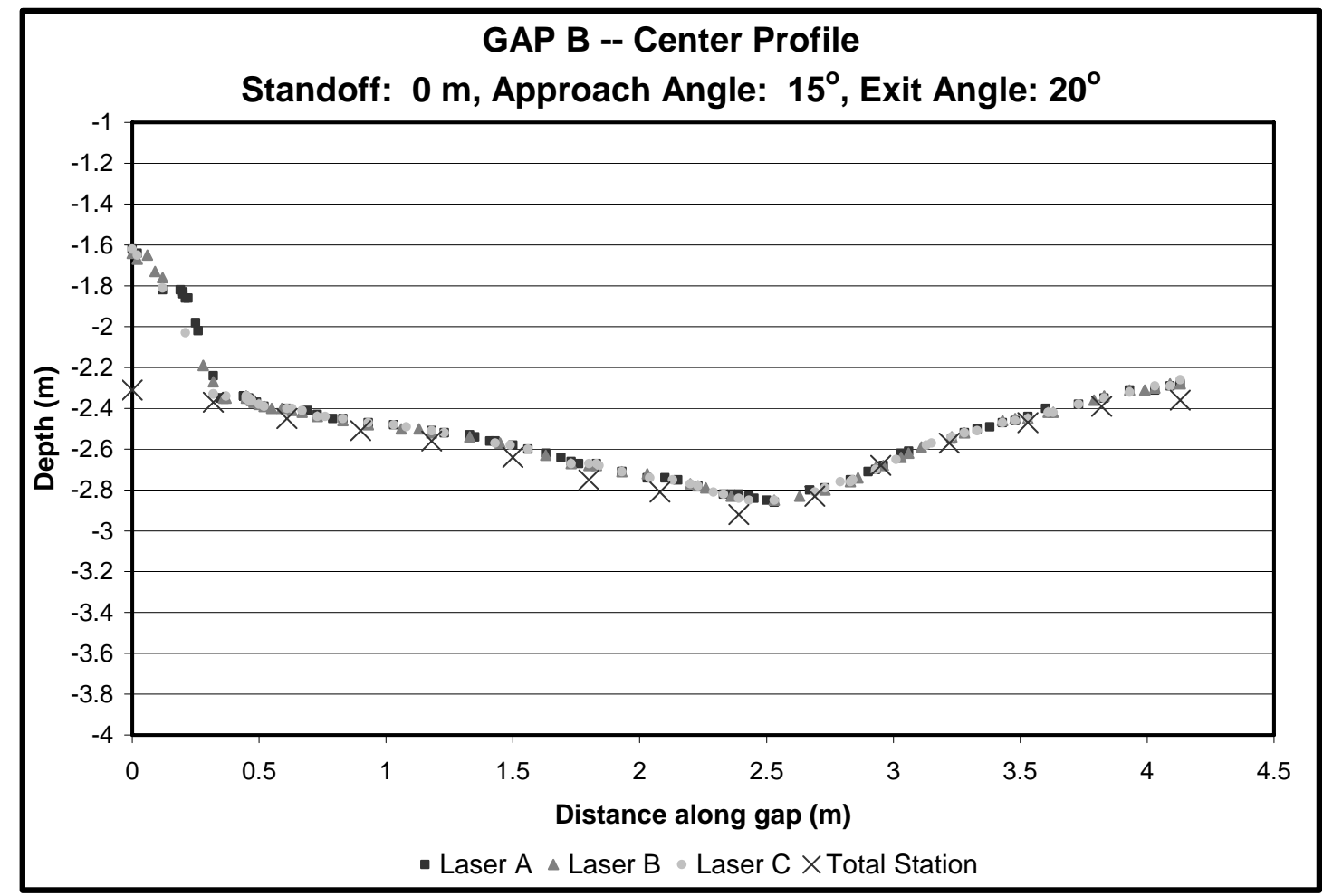

Figure 53. Data for Gap B center profile. 


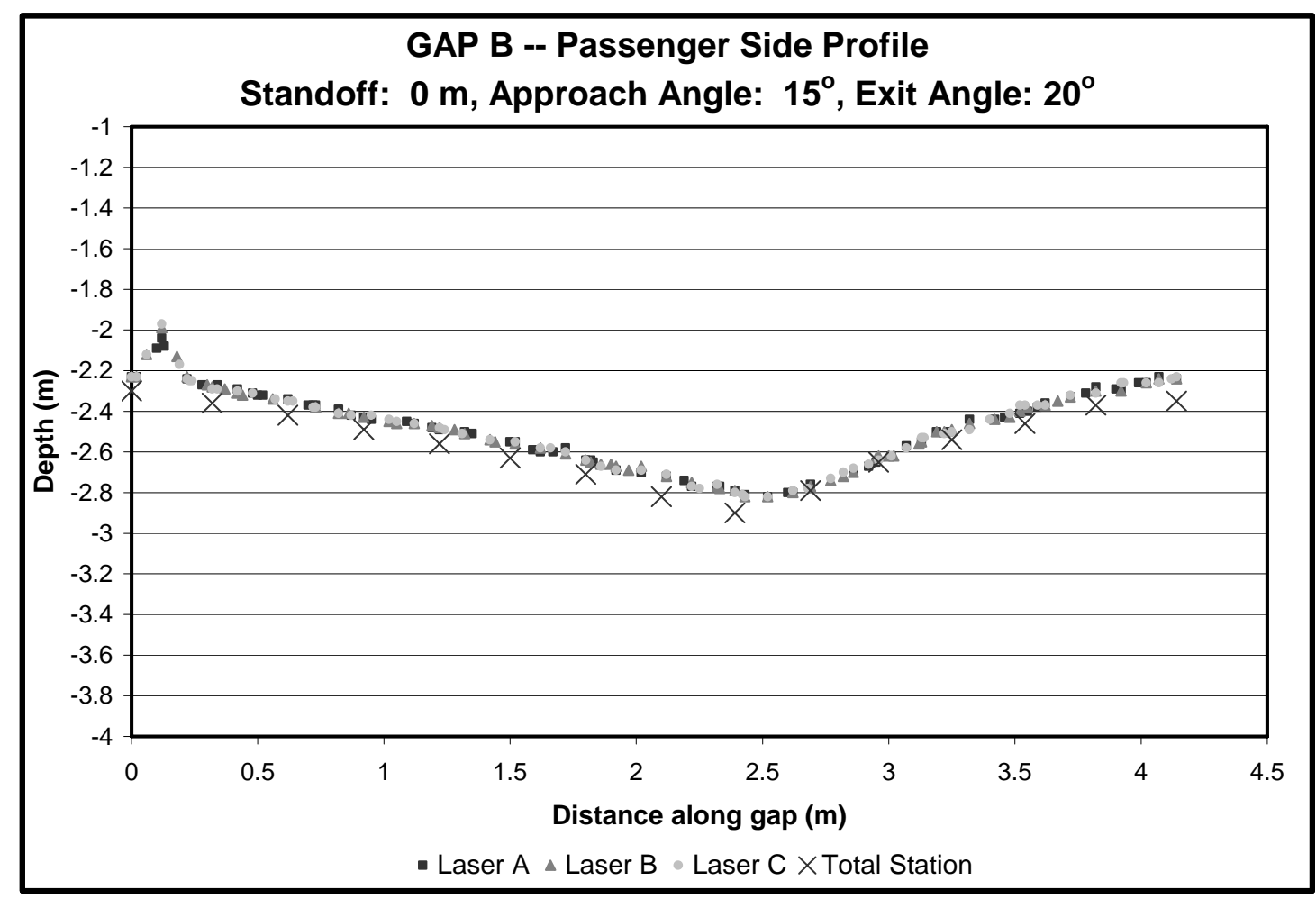

Figure 54. Data for Gap B - passenger side profile.

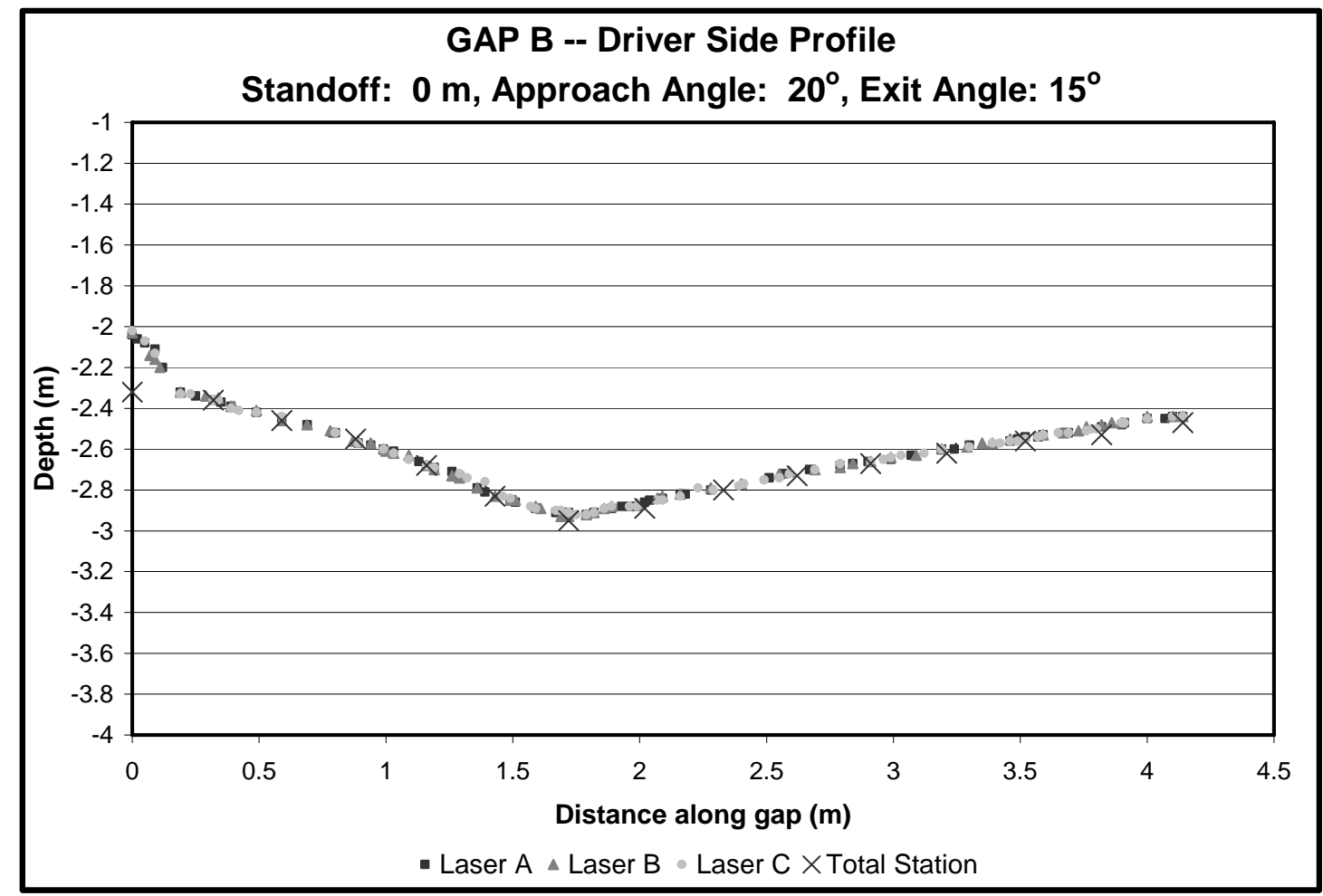

Figure 55. Data for Gap B - driver side profile. 


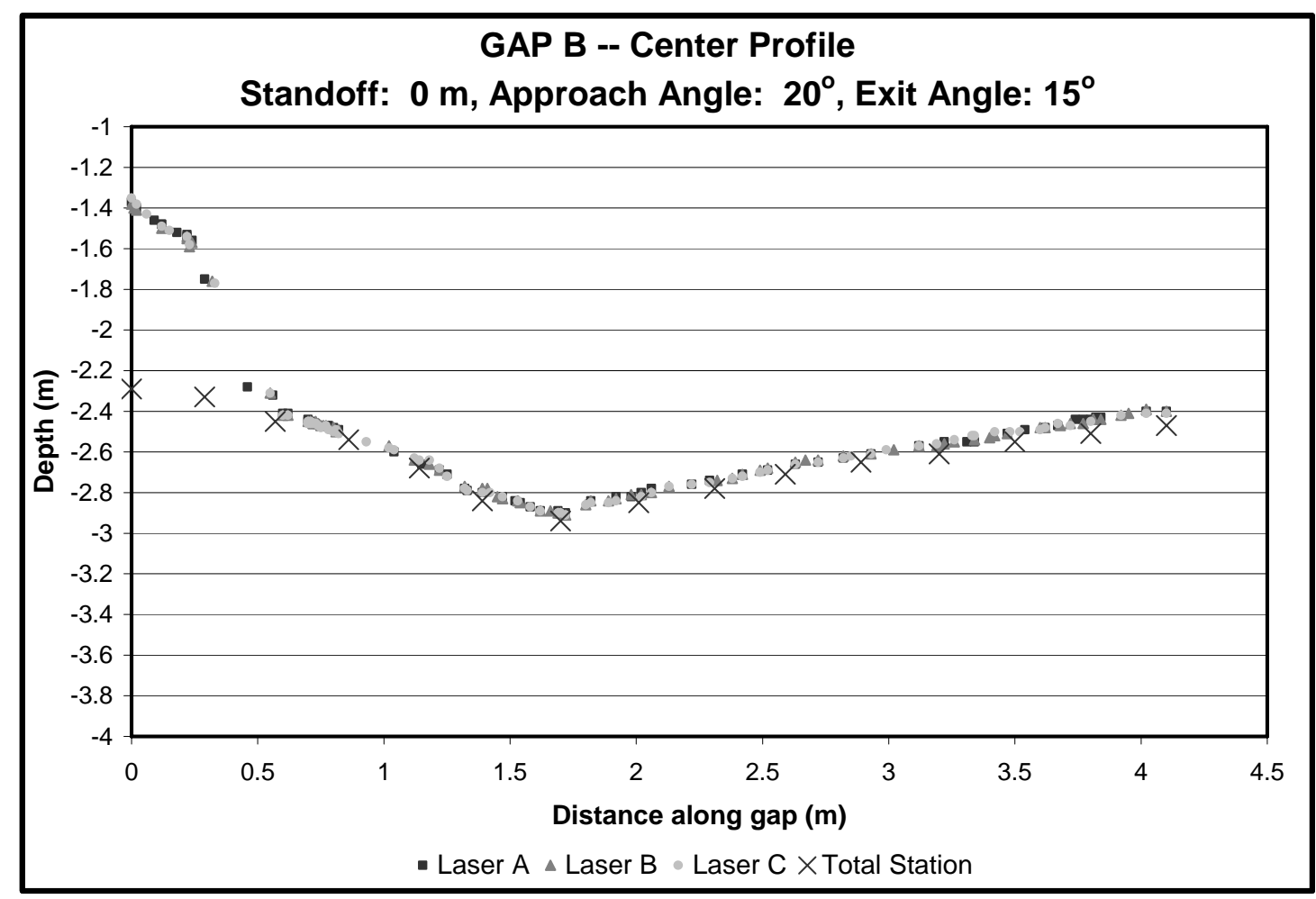

Figure 56. Data for Gap B - center profile.

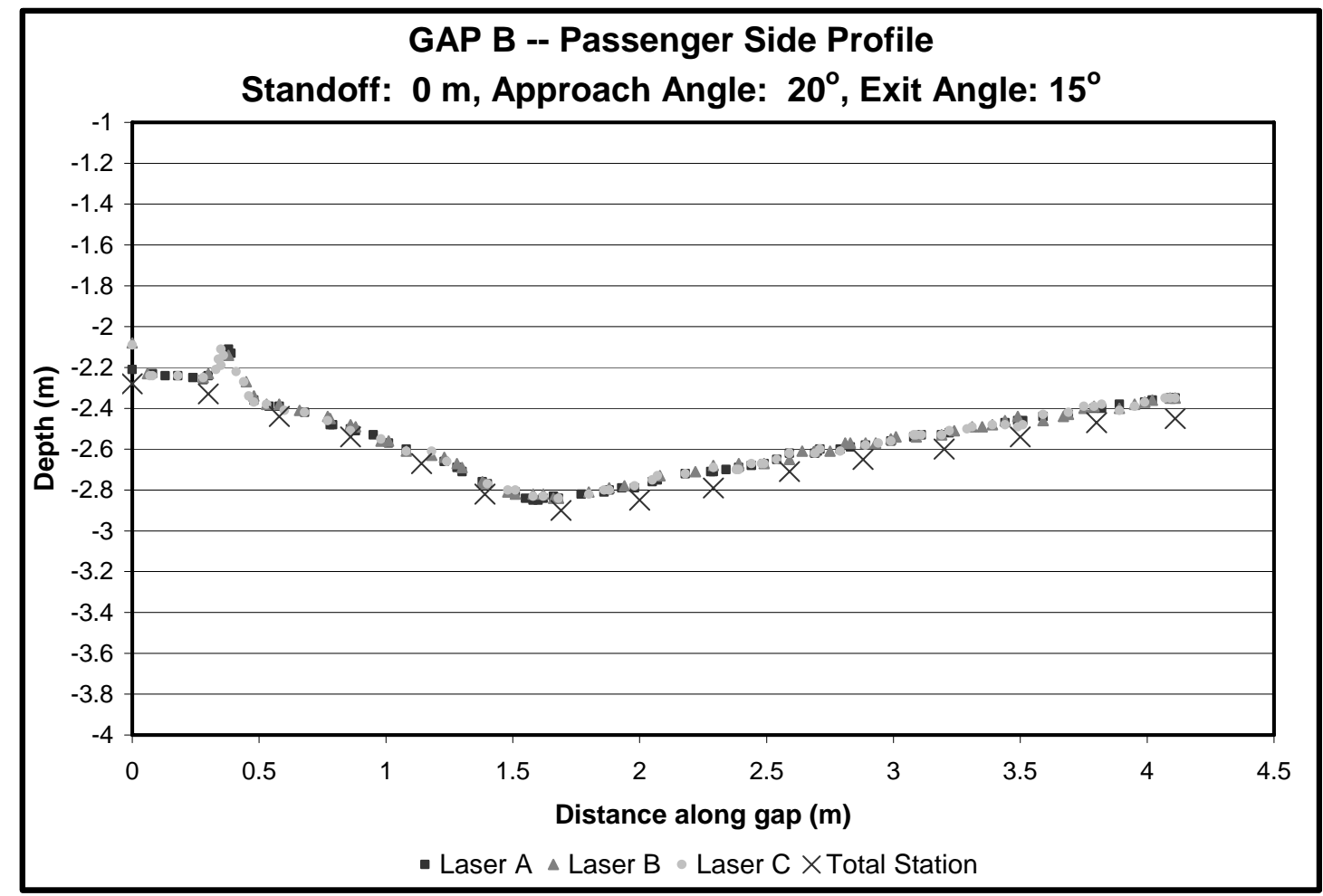

Figure 57. Data for Gap B - passenger side profile. 


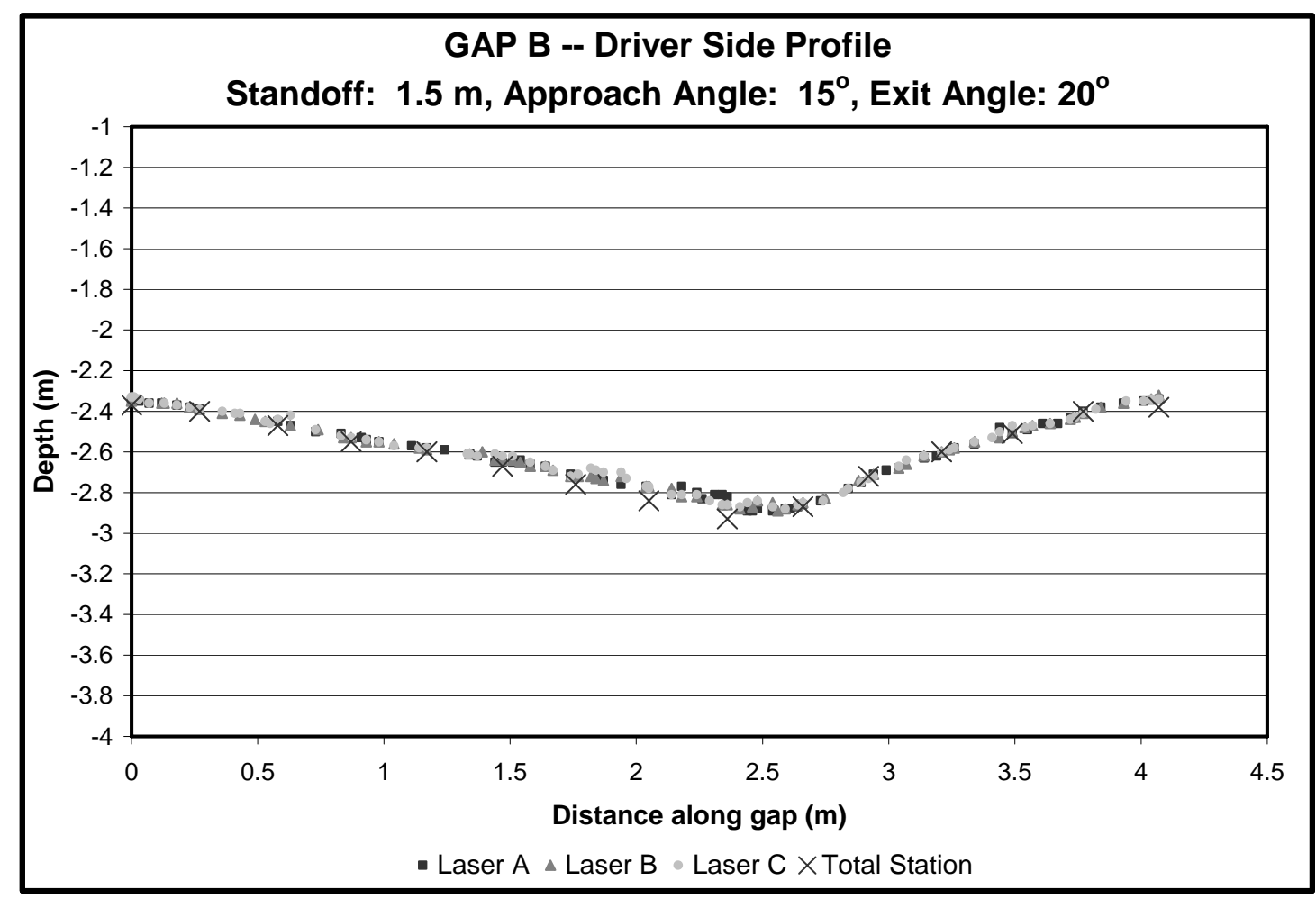

Figure 58. Data for Gap B - driver side profile.

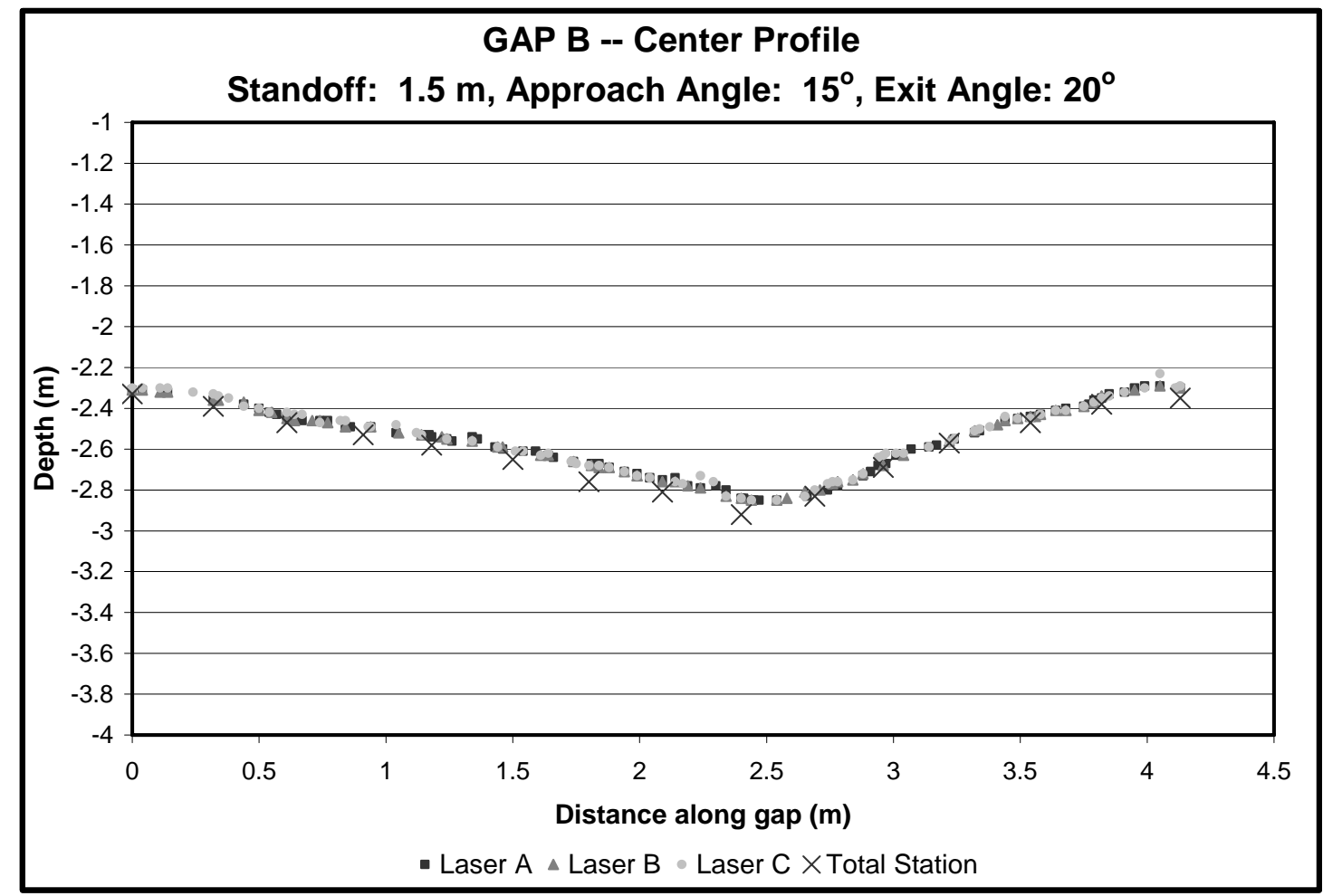

Figure 59. Data for Gap B - center profile. 


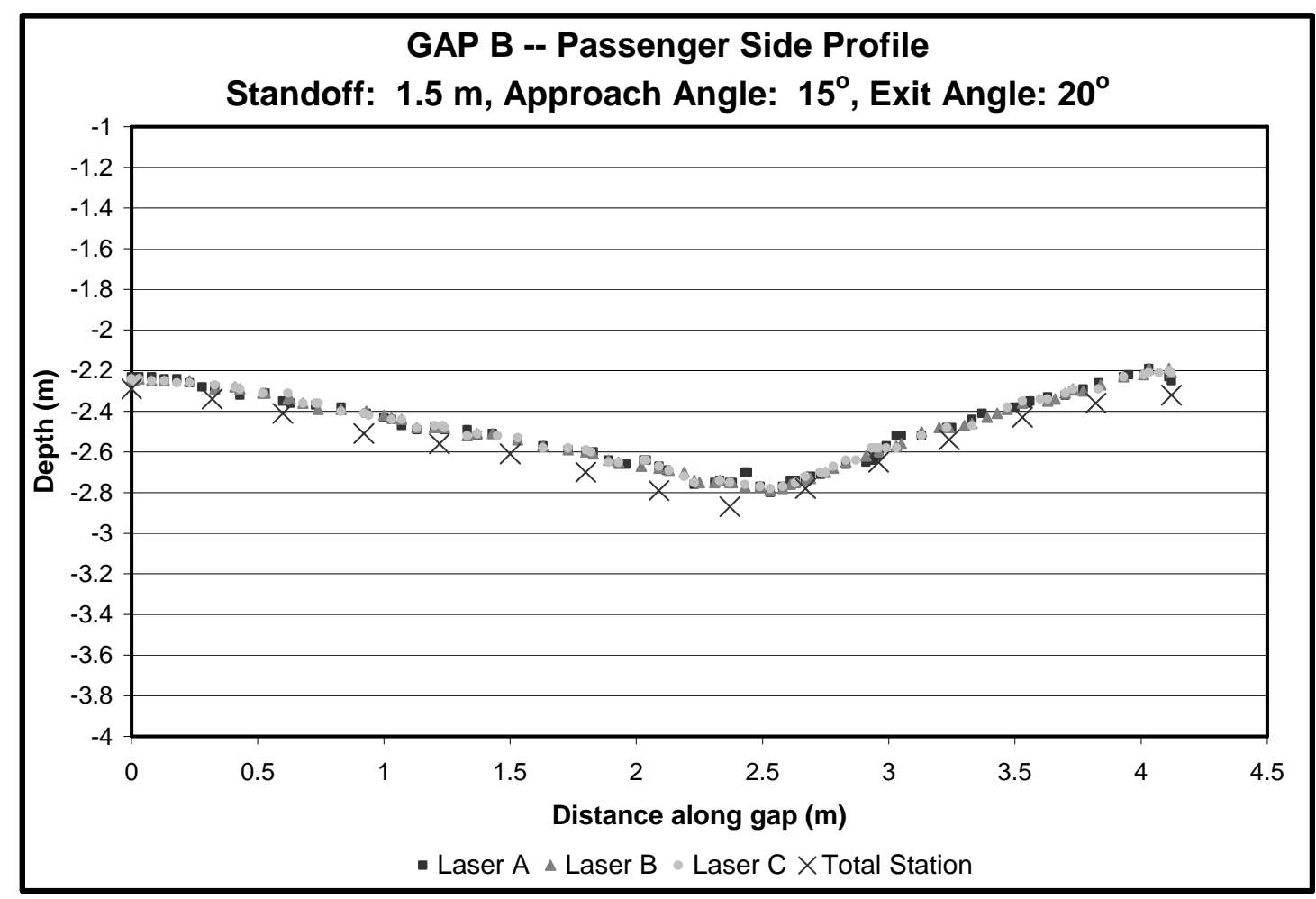

Figure 60. Data for Gap B - passenger side profile.

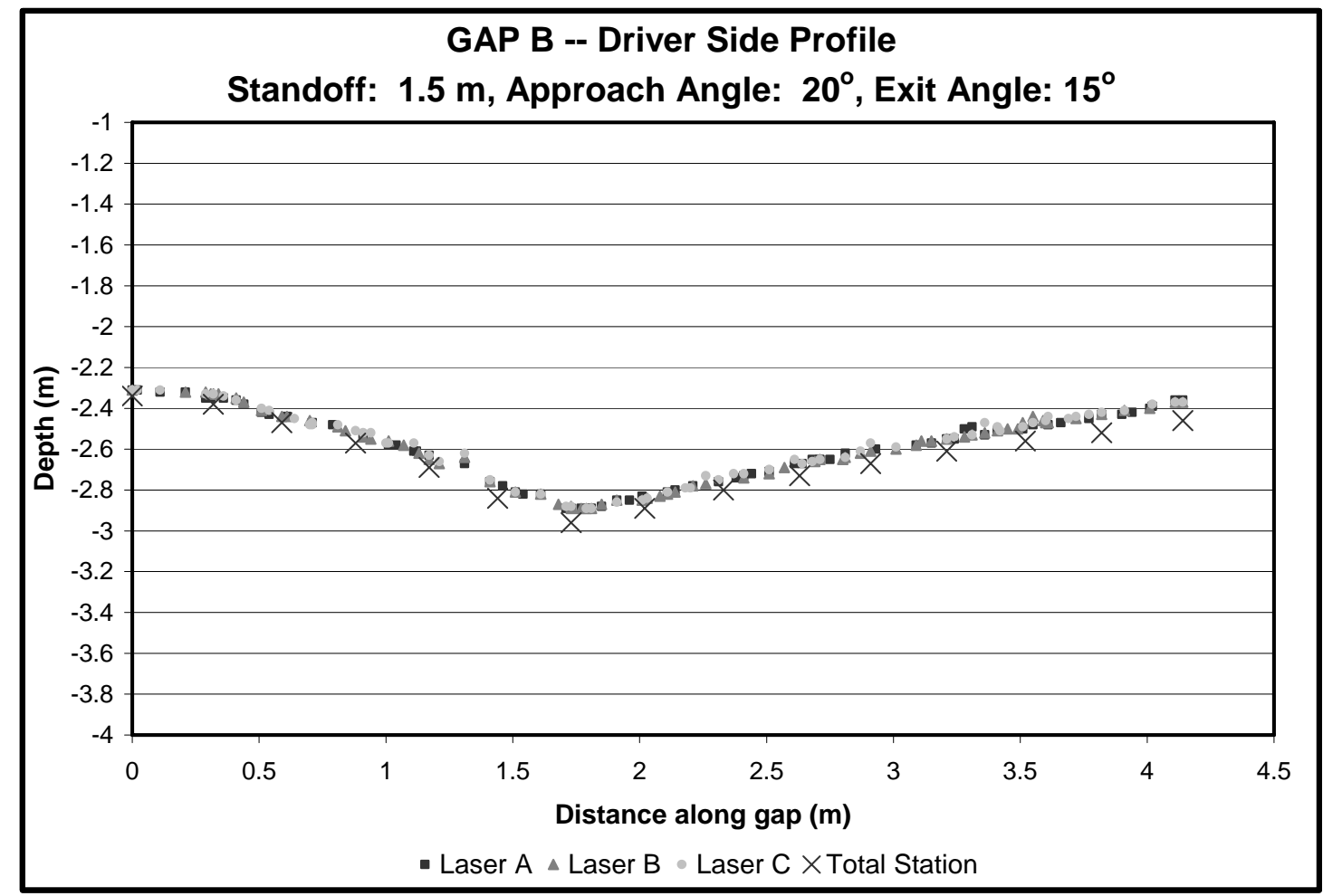

Figure 61. Data for Gap B - driver side profile. 


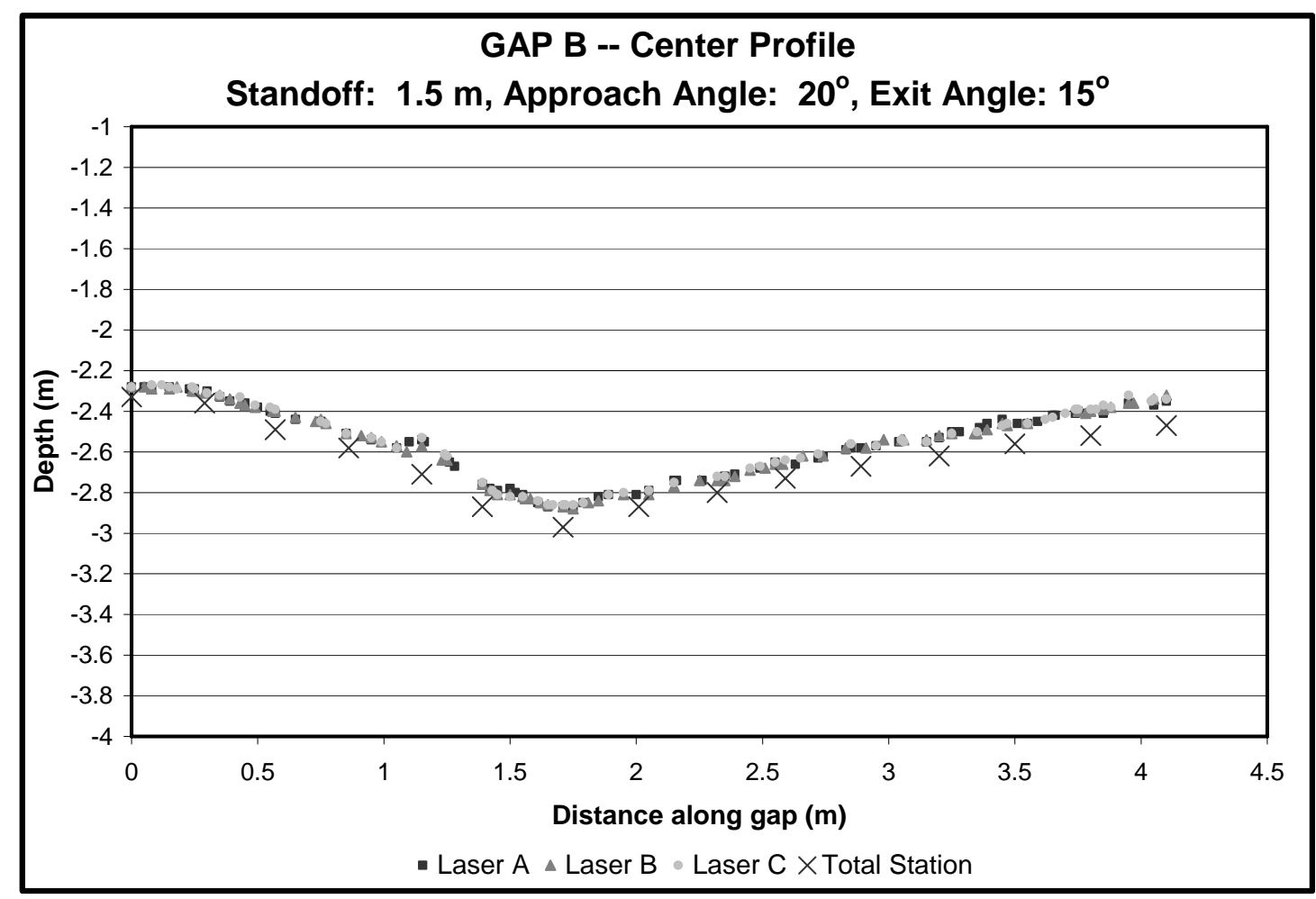

Figure 62. Data for Gap B - center profile.

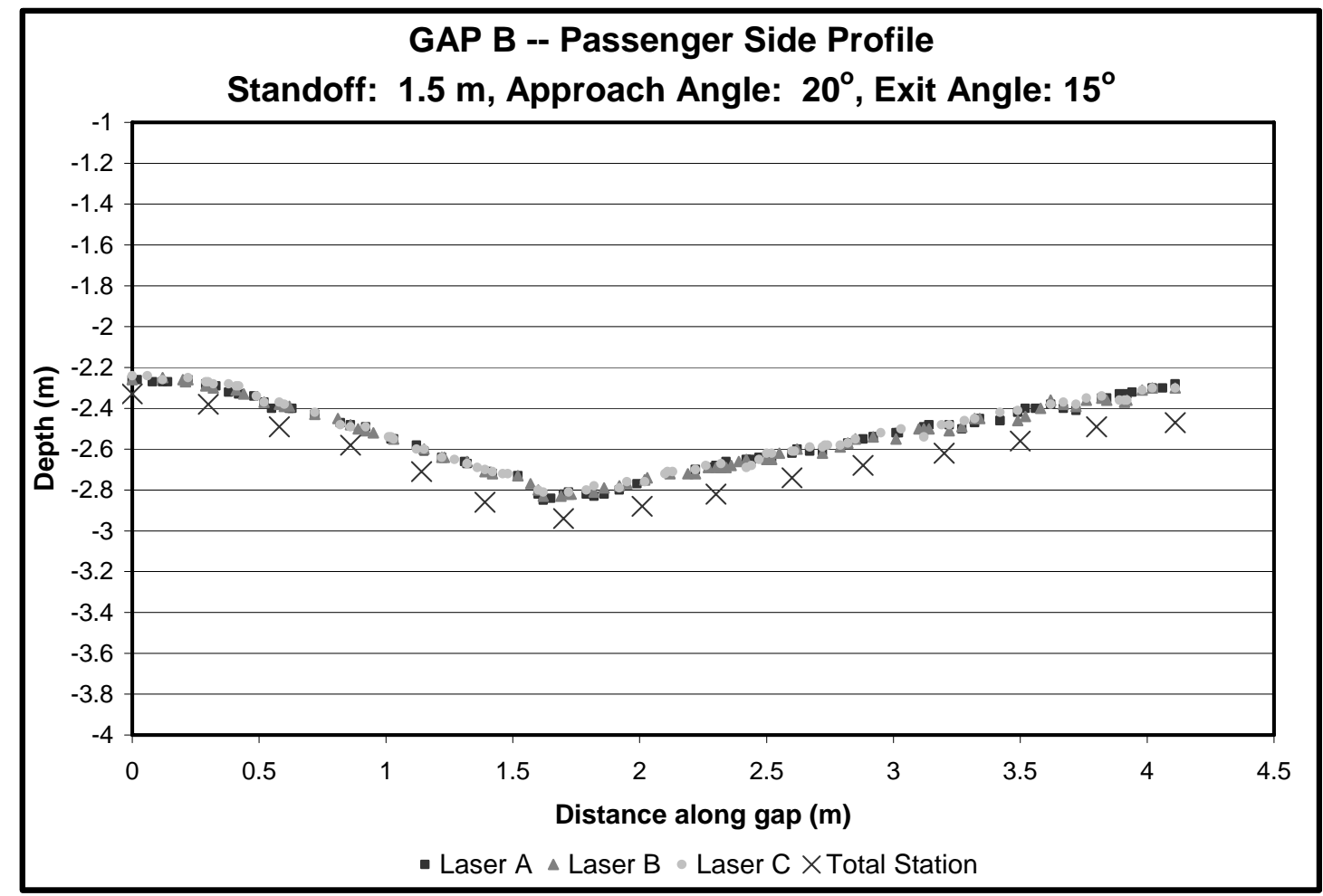

Figure 63. Data for Gap B - passenger side profile. 


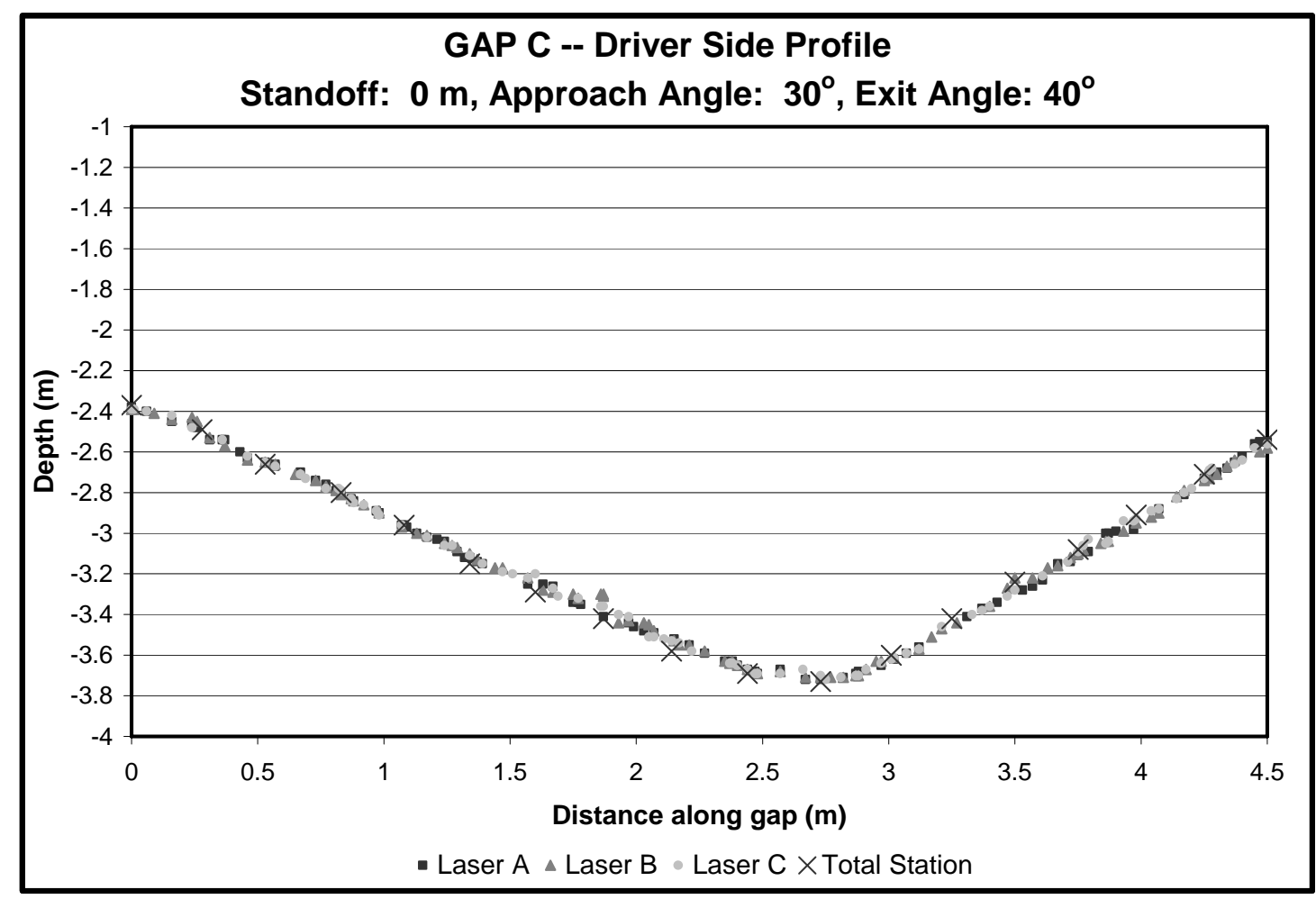

Figure 64. Data for Gap C - driver side profile.

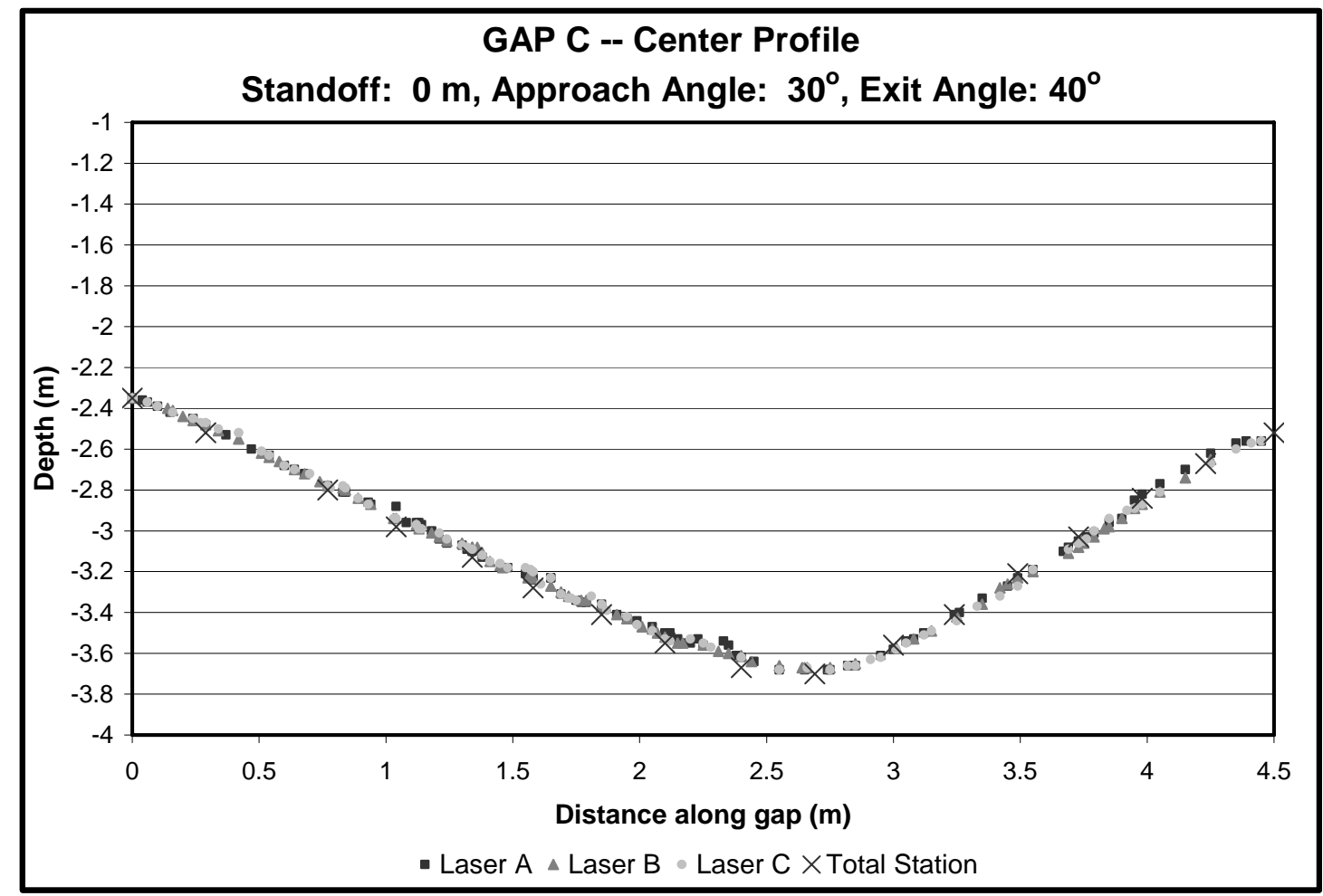

Figure 65. Data for Gap C - center profile. 


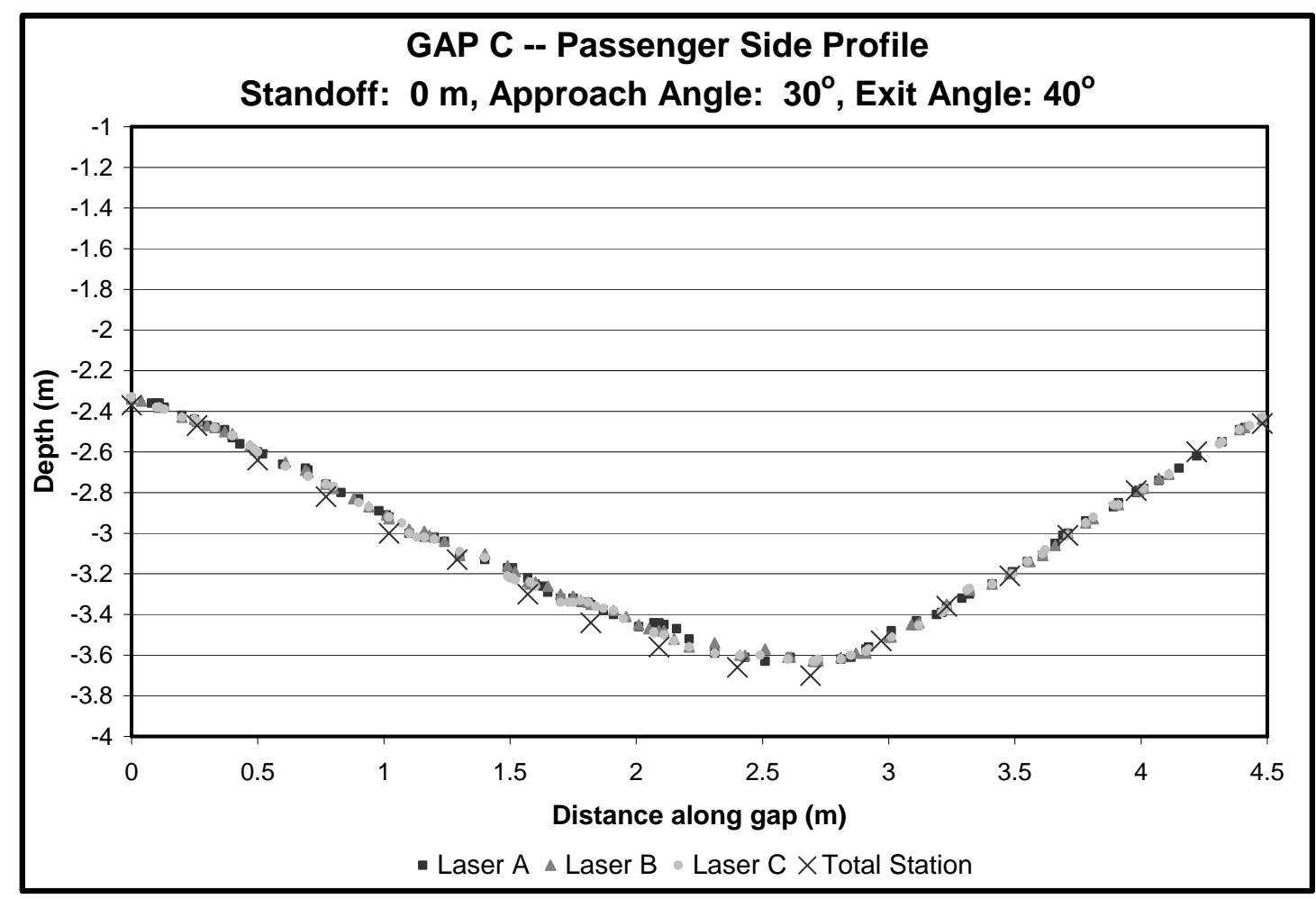

Figure 66. Data for Gap C - passenger side profile.

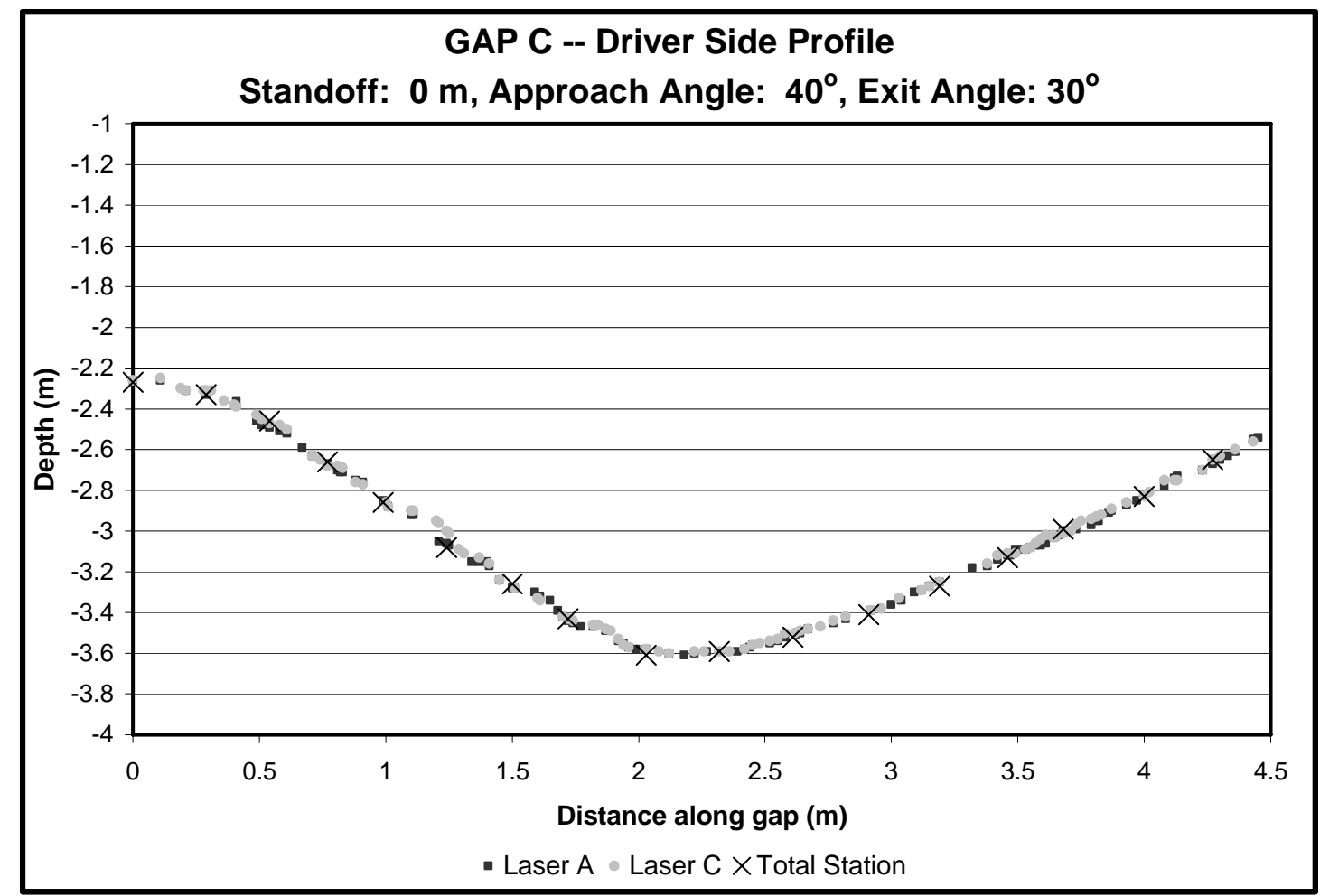

Figure 67. Data for Gap C - driver side profile. 


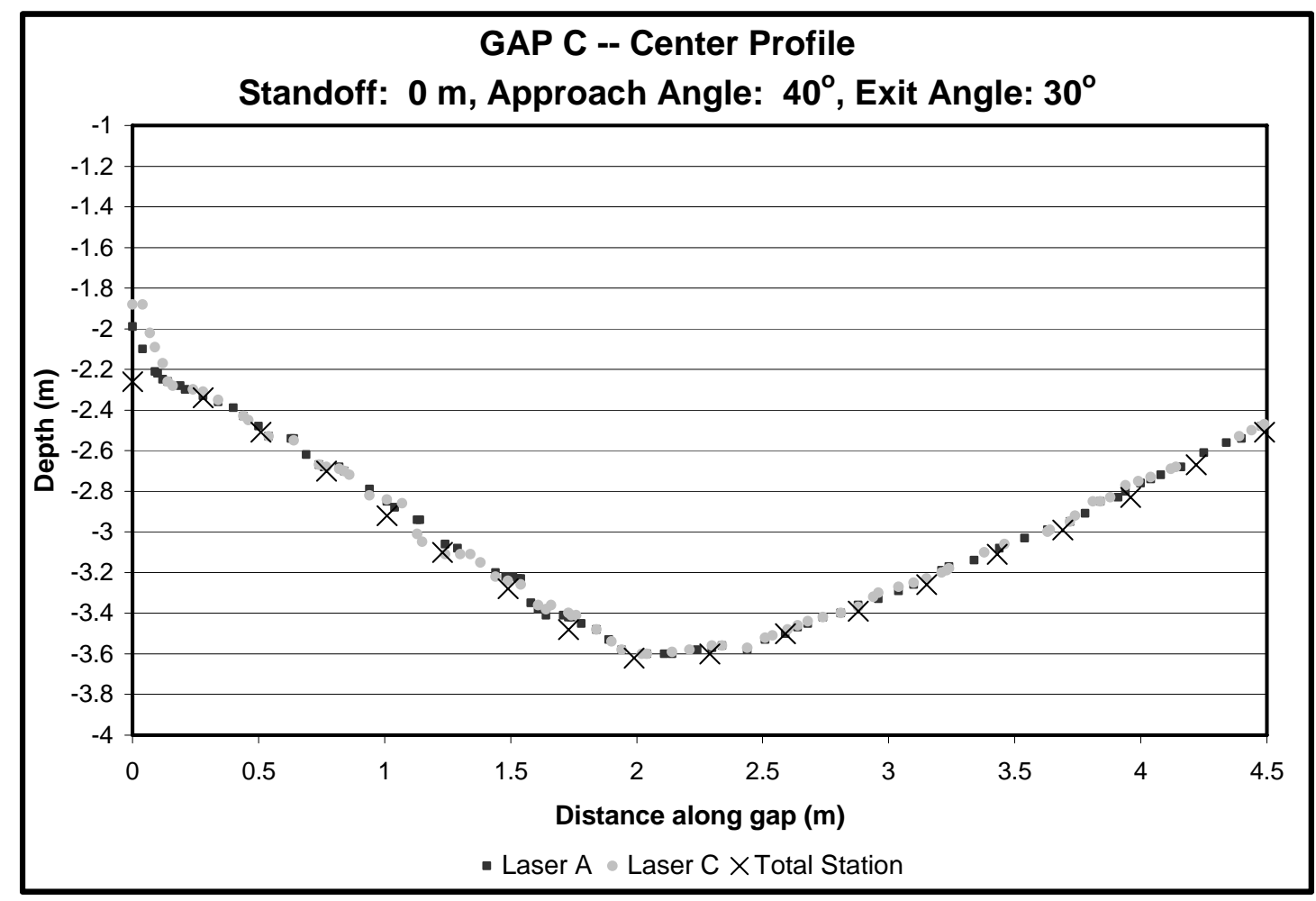

Figure 68. Data for Gap C - center profile.

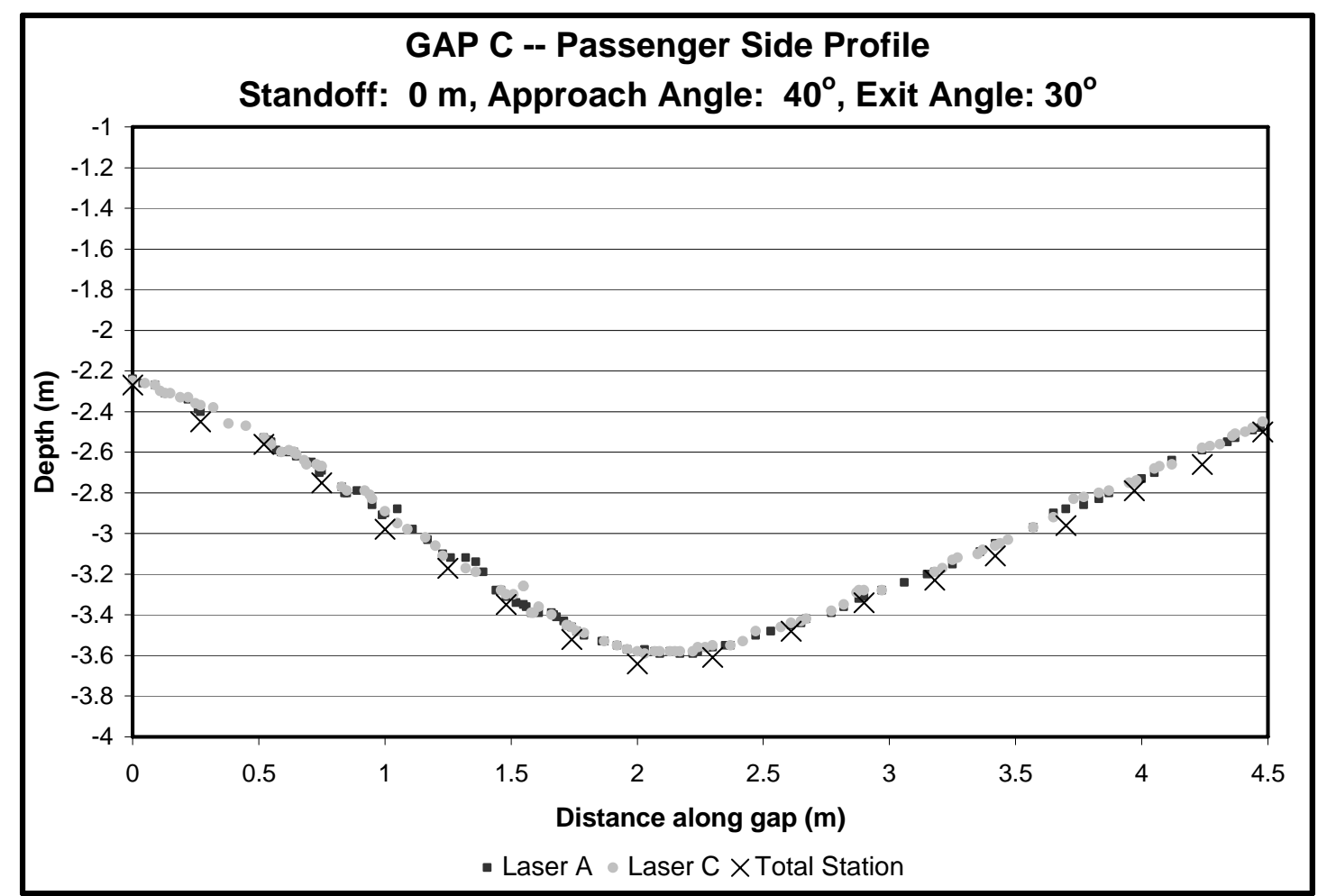

Figure 69. Data for Gap C - passenger side profile. 


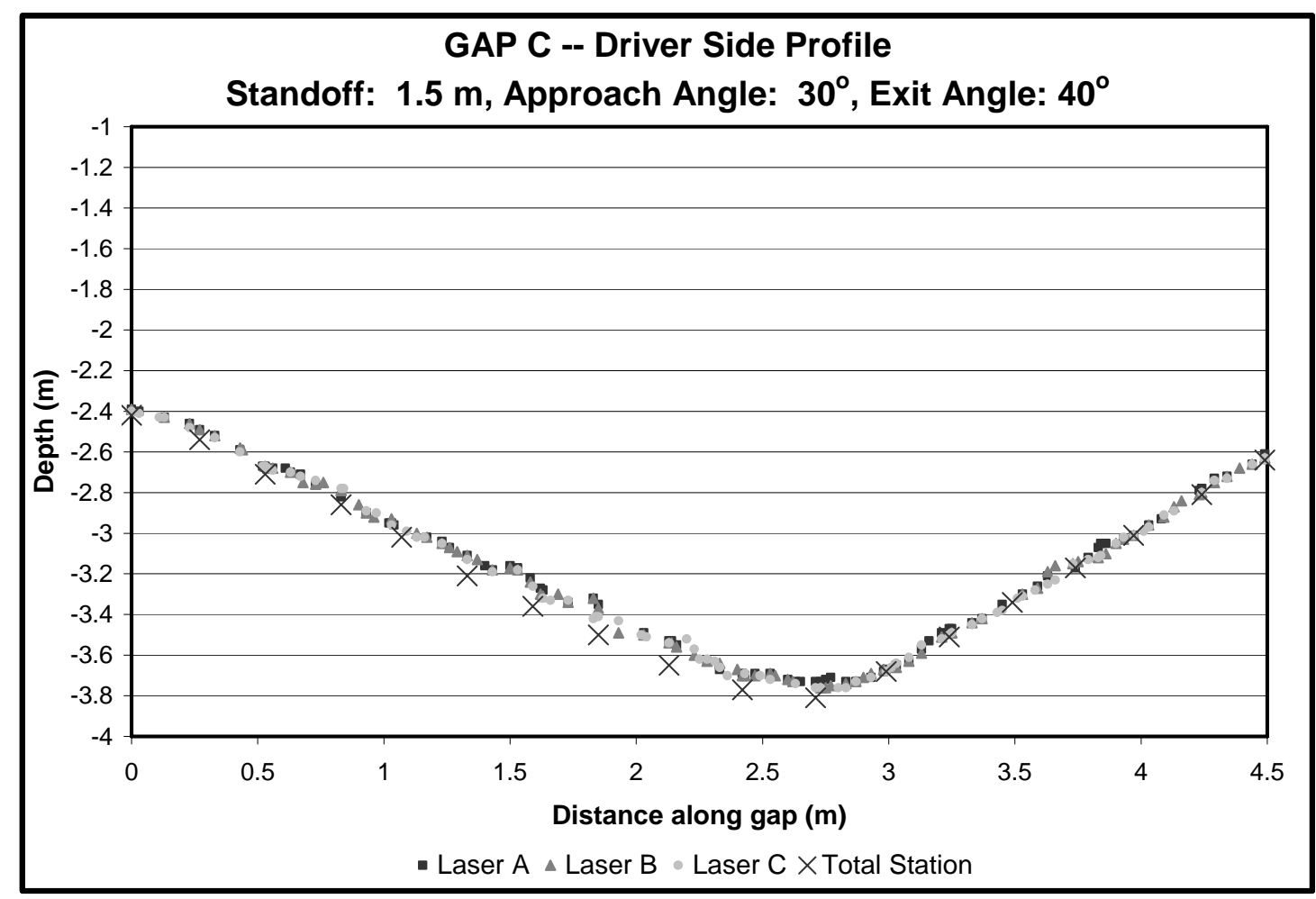

Figure 70. Data for Gap C - driver side profile.

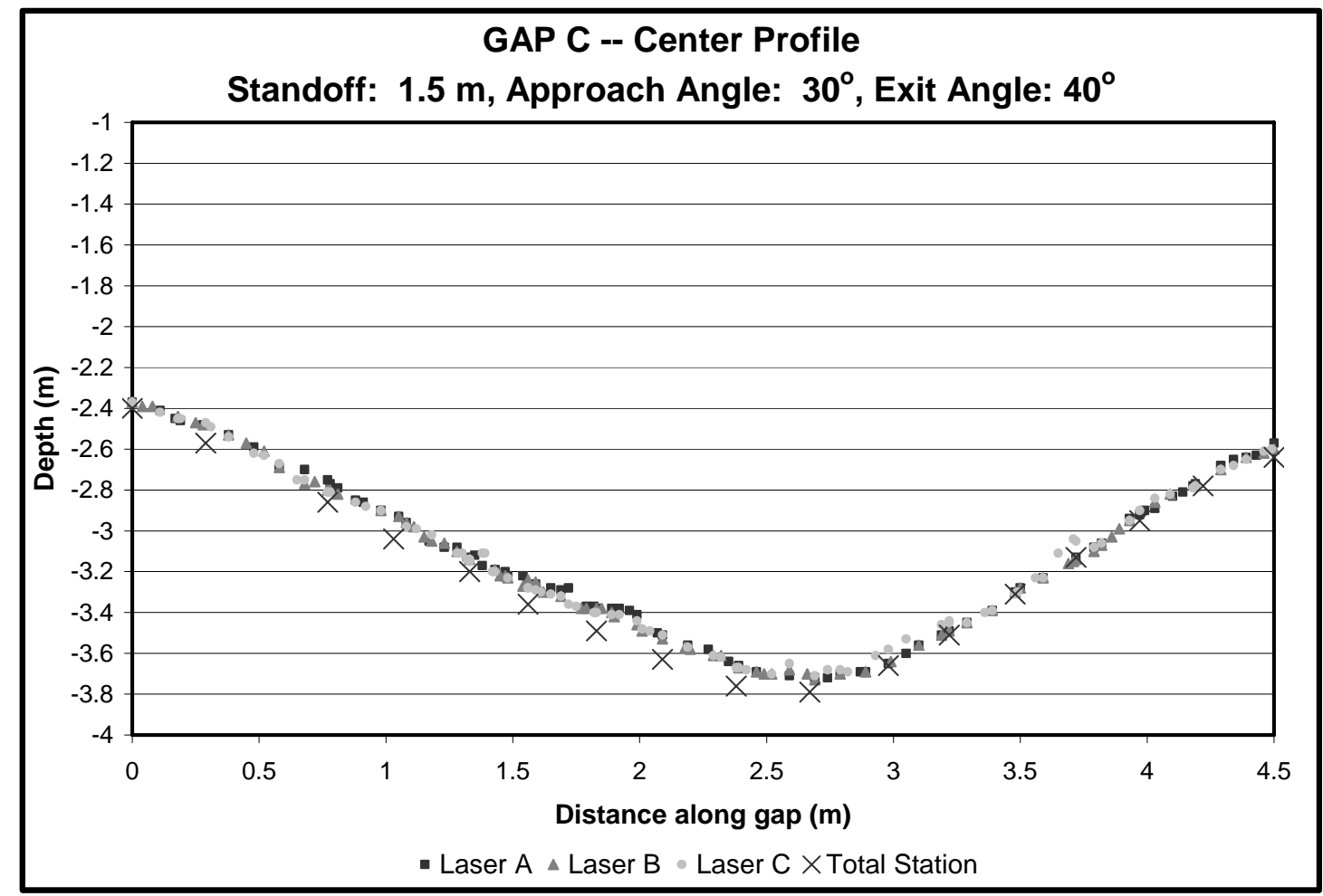

Figure 71. Data for Gap C - center profile. 


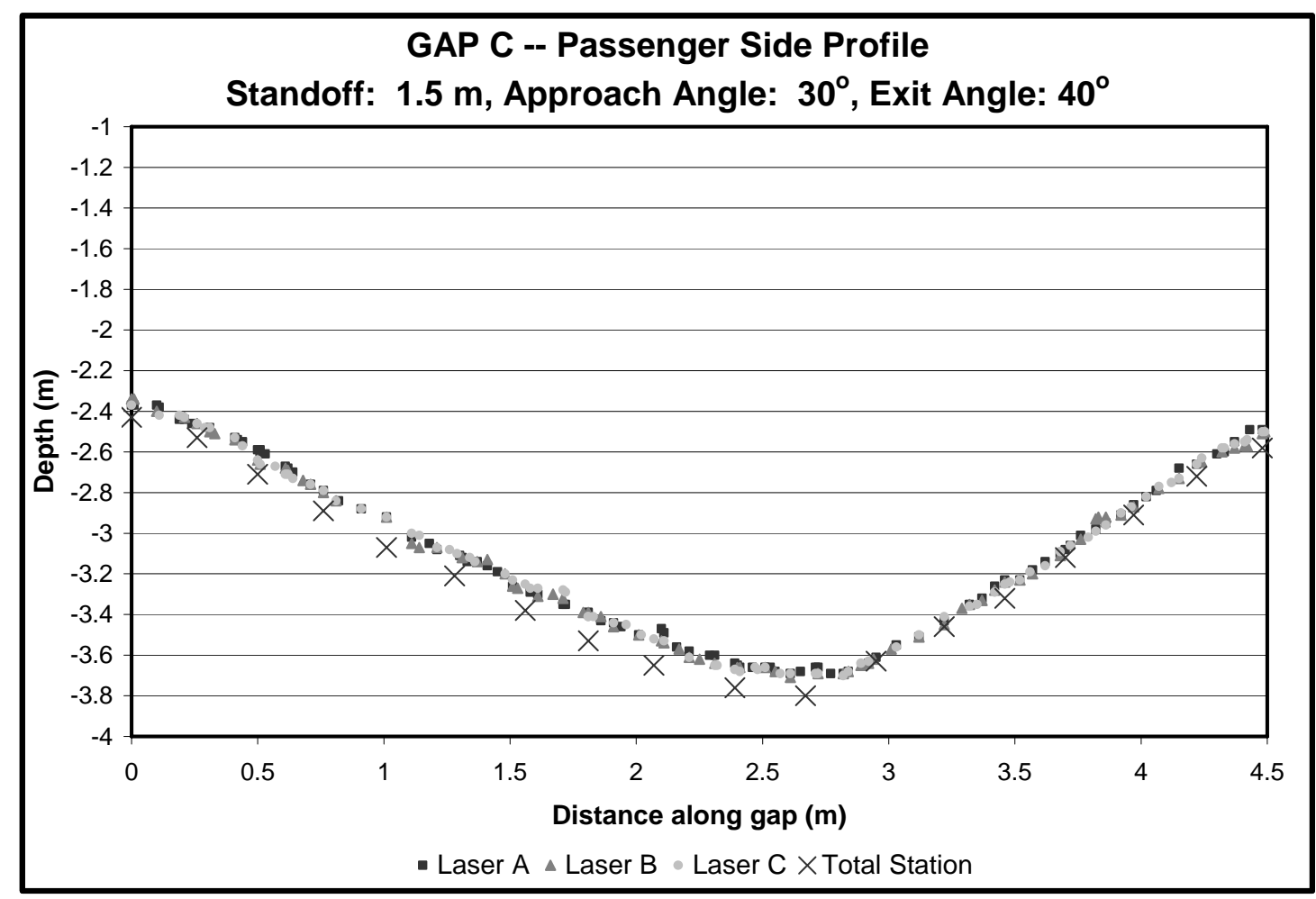

Figure 72. Data for Gap C - passenger side profile.

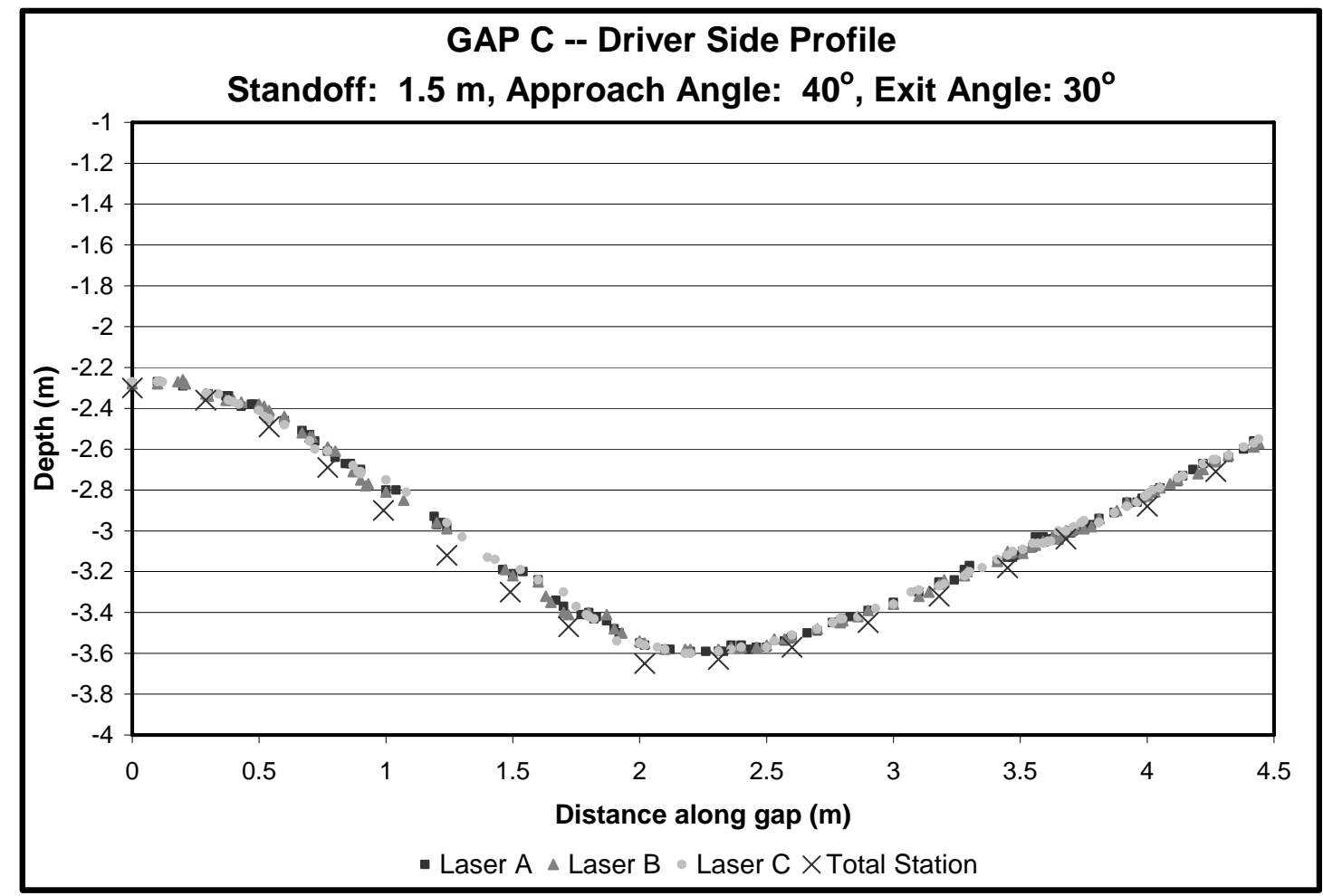

Figure 73. Data for Gap C - driver side profile. 


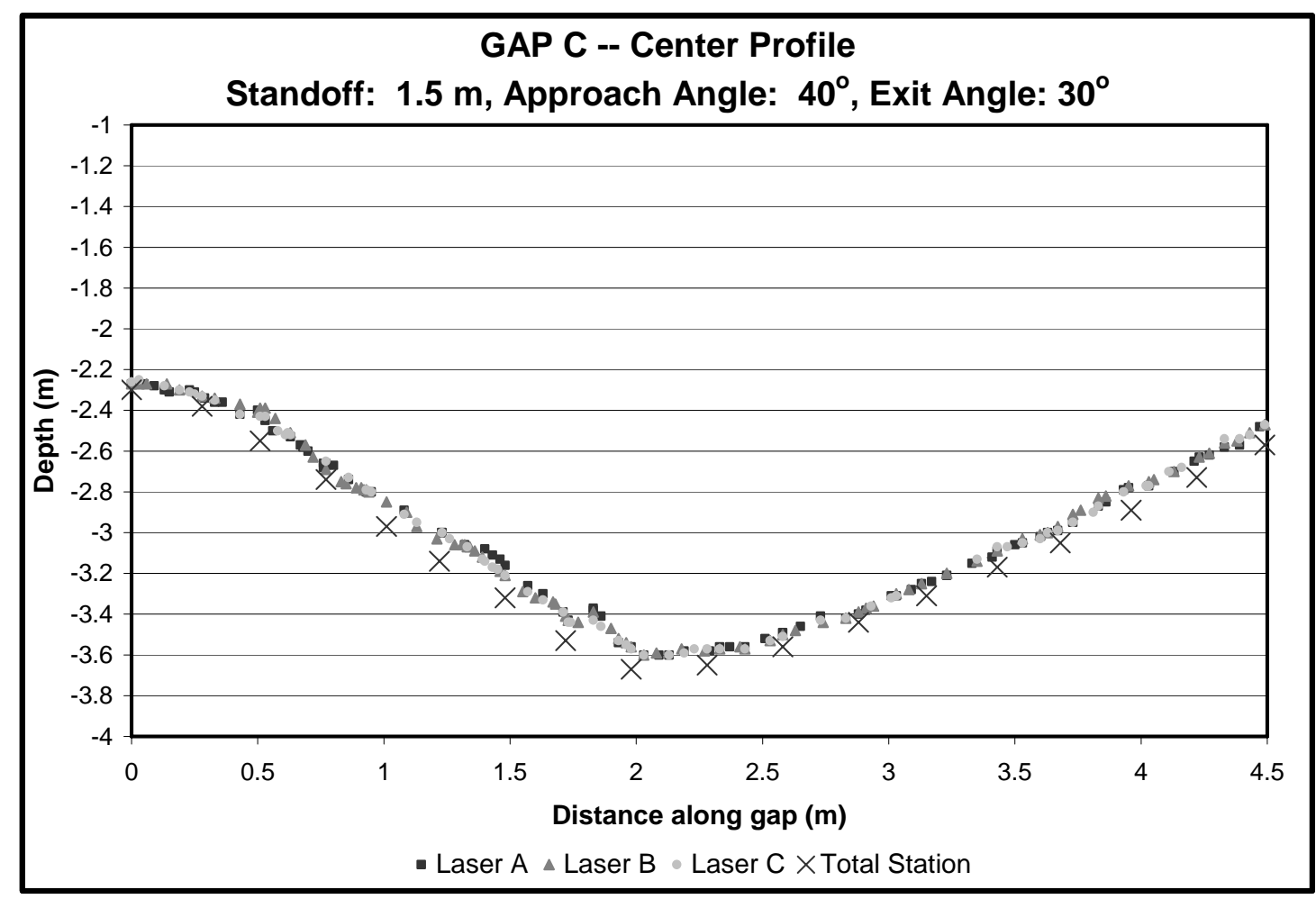

Figure 74. Data for Gap C - center profile.

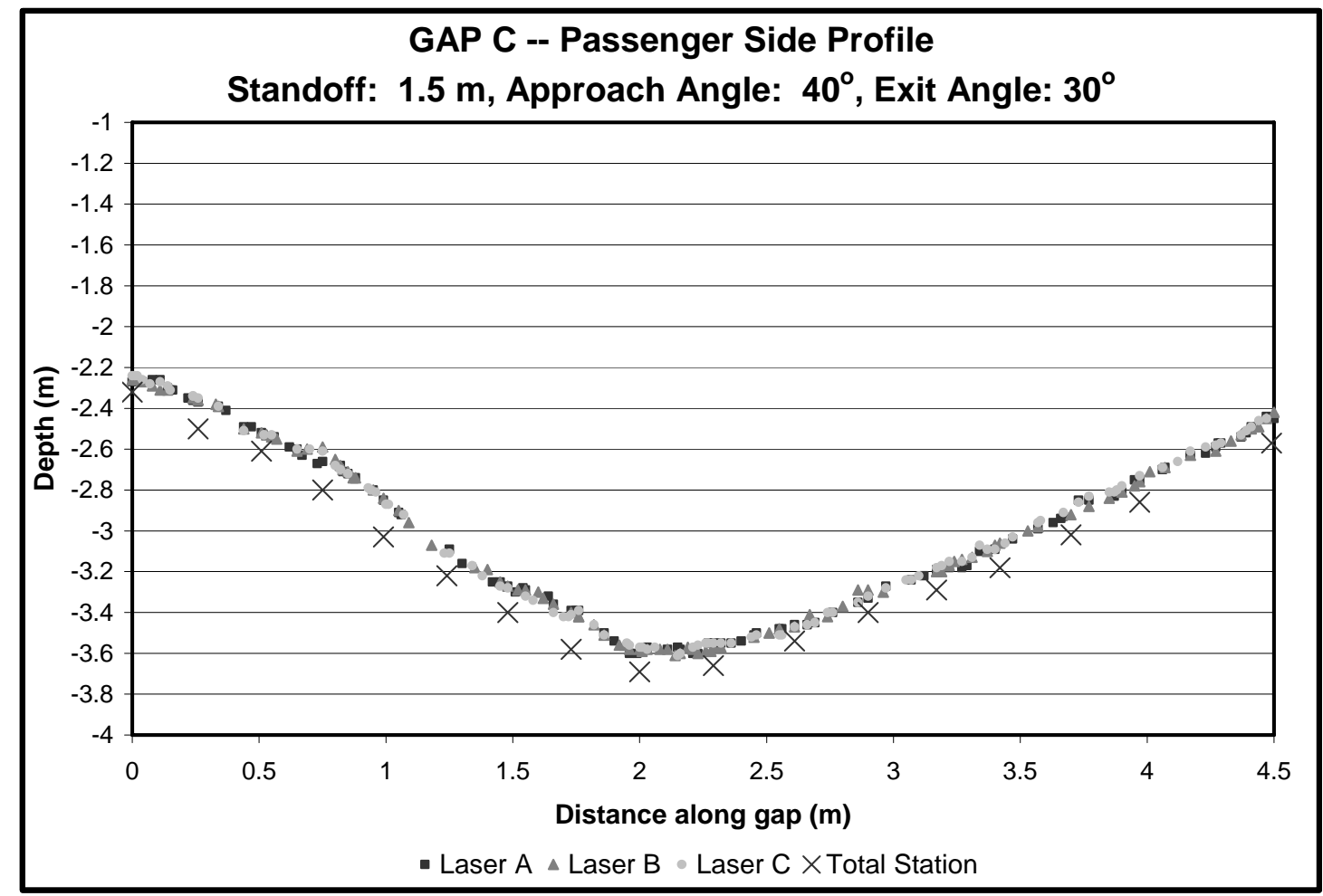

Figure 75. Data for Gap C - passenger side profile. 


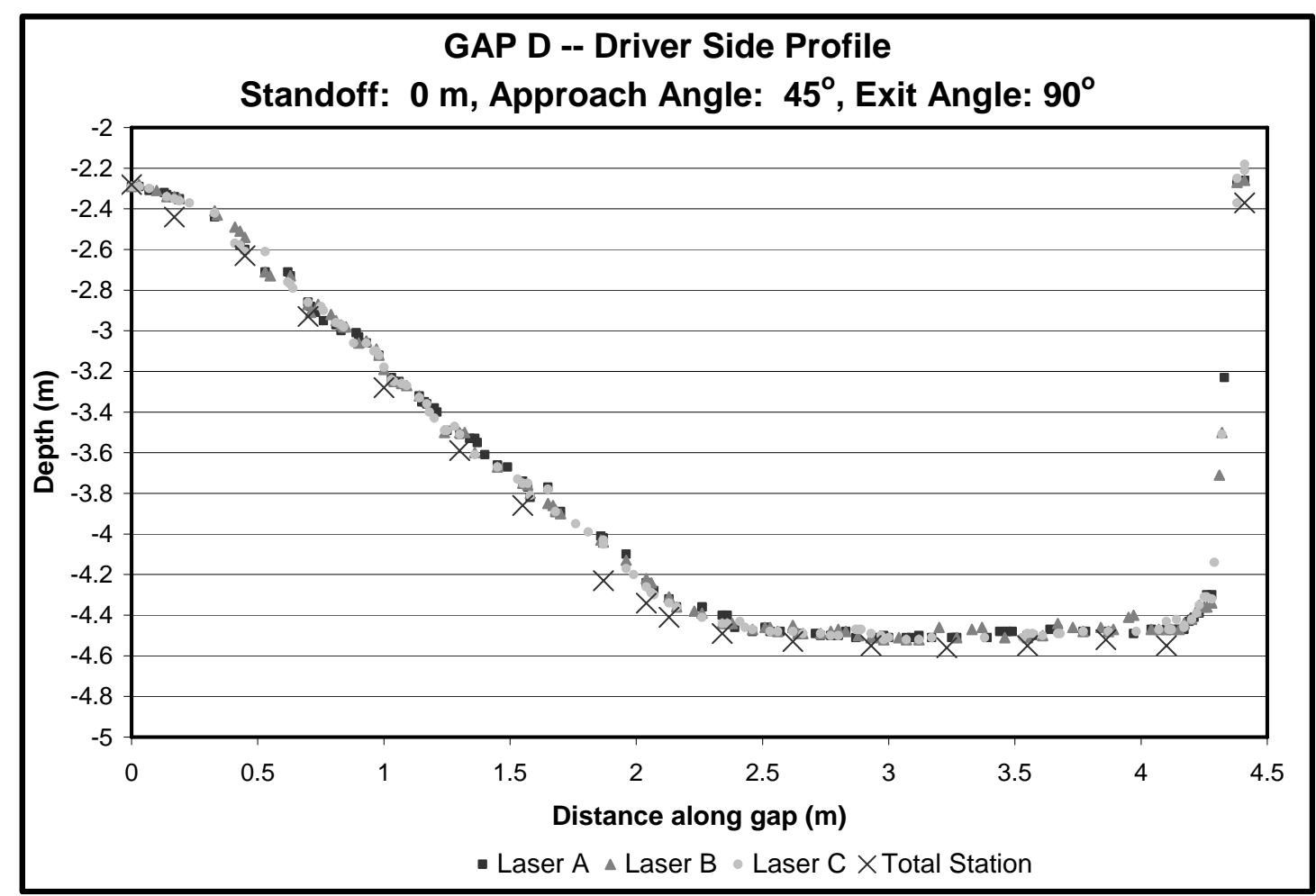

Figure 76. Data for Gap D - driver side profile.

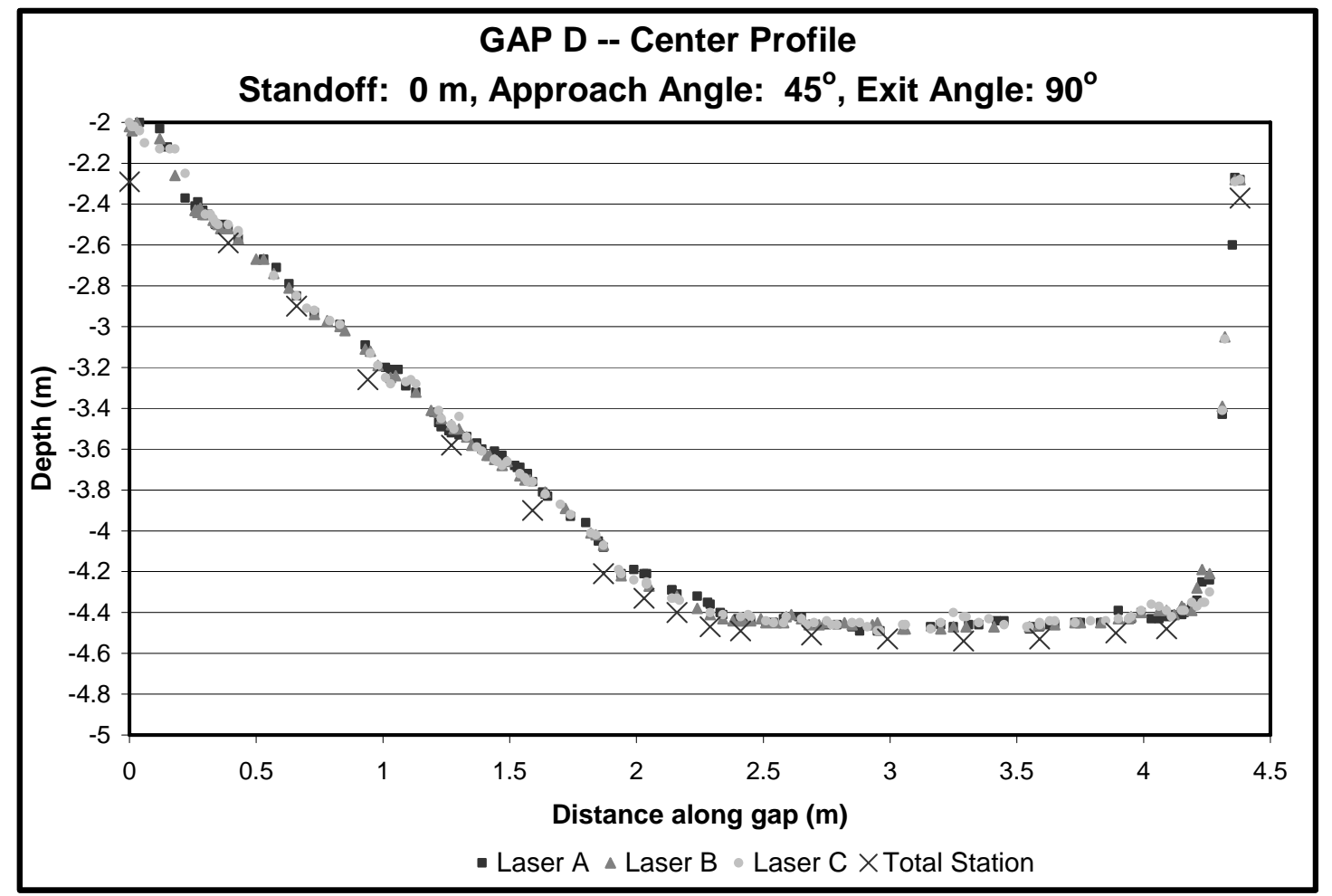

Figure 77. Data for Gap D - center profile. 


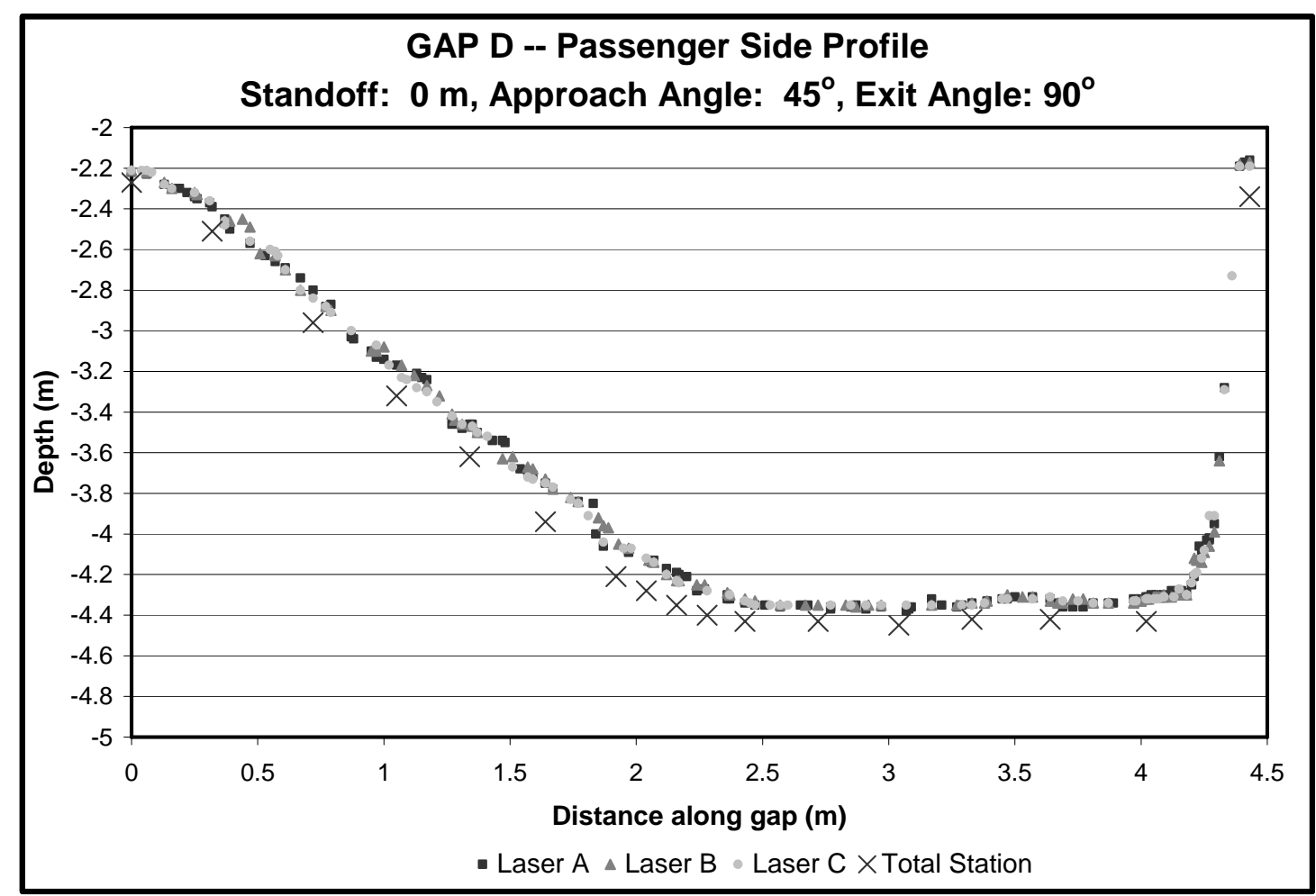

Figure 78. Data for Gap D - passenger side profile.

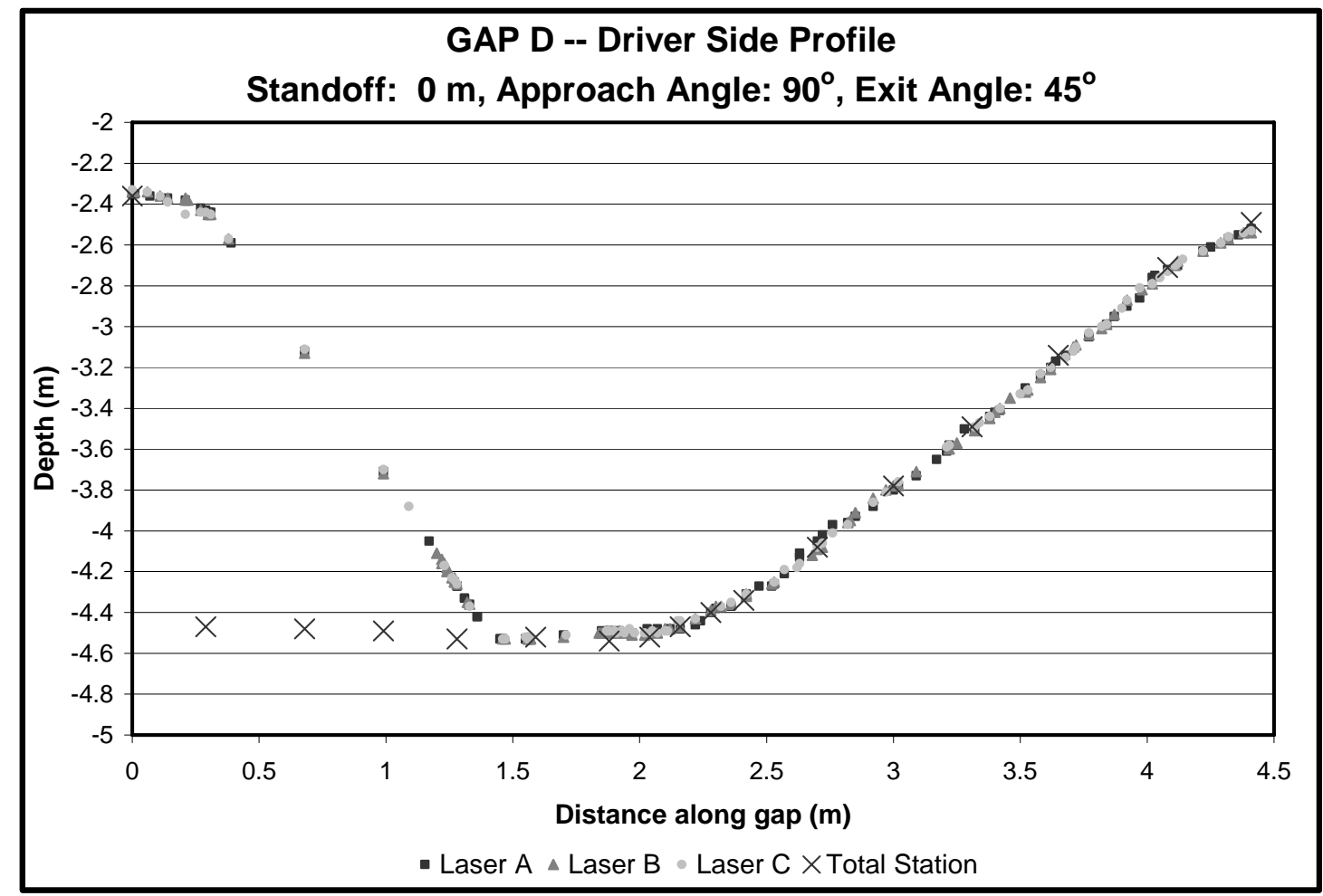

Figure 79. Data for Gap D - driver side profile. 


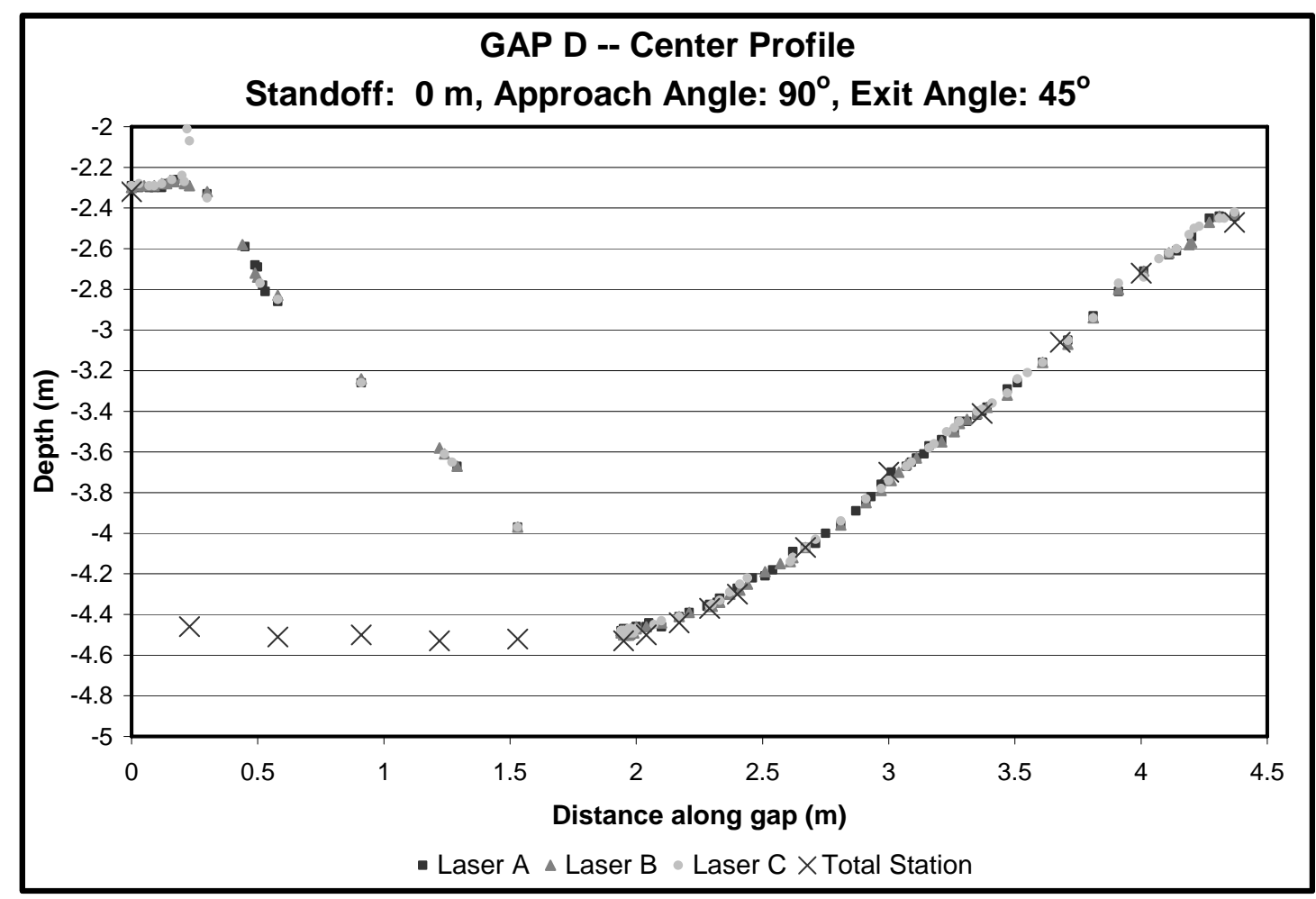

Figure 80. Data for Gap D - center profile.

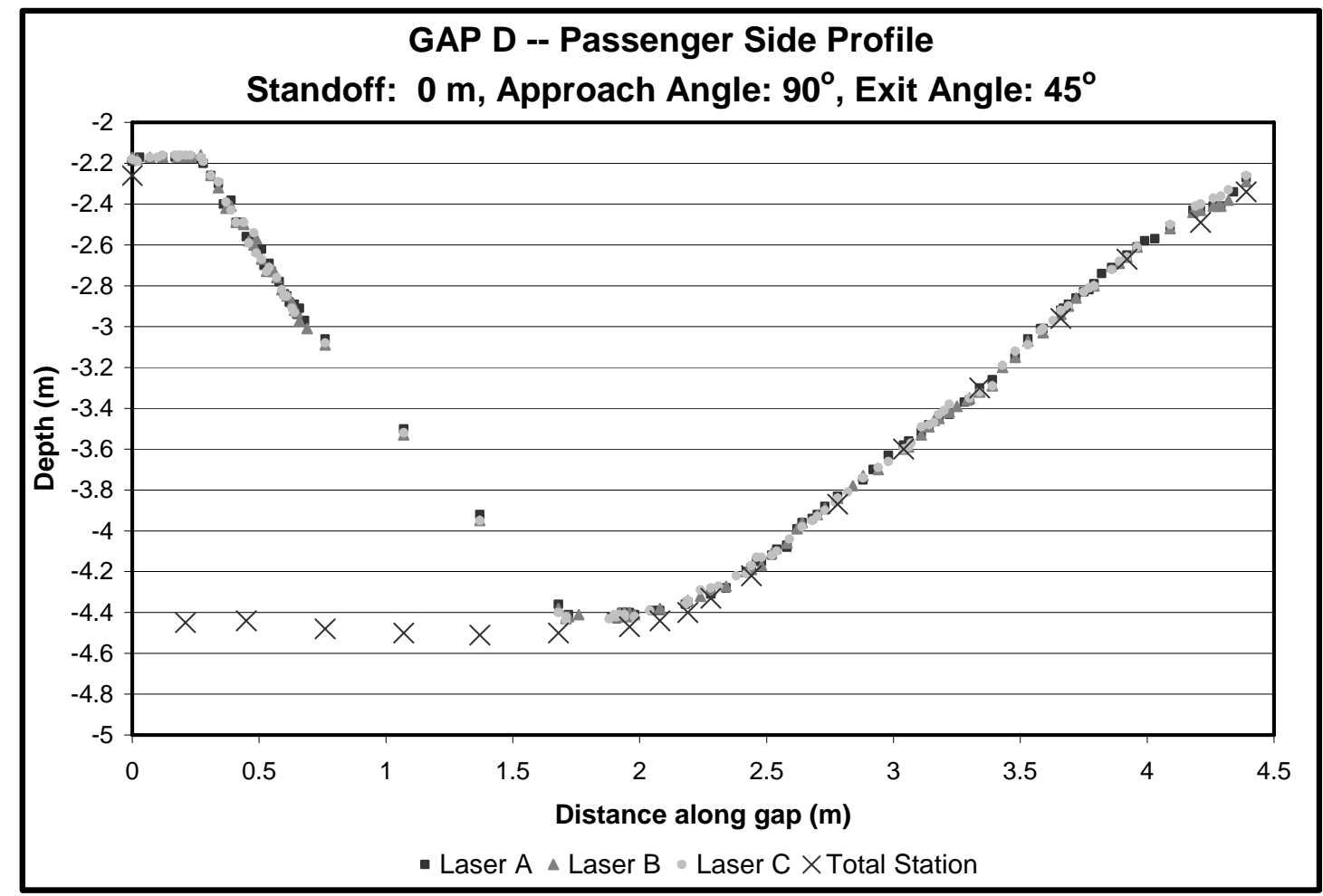

Figure 81. Data for Gap D - passenger side profile. 


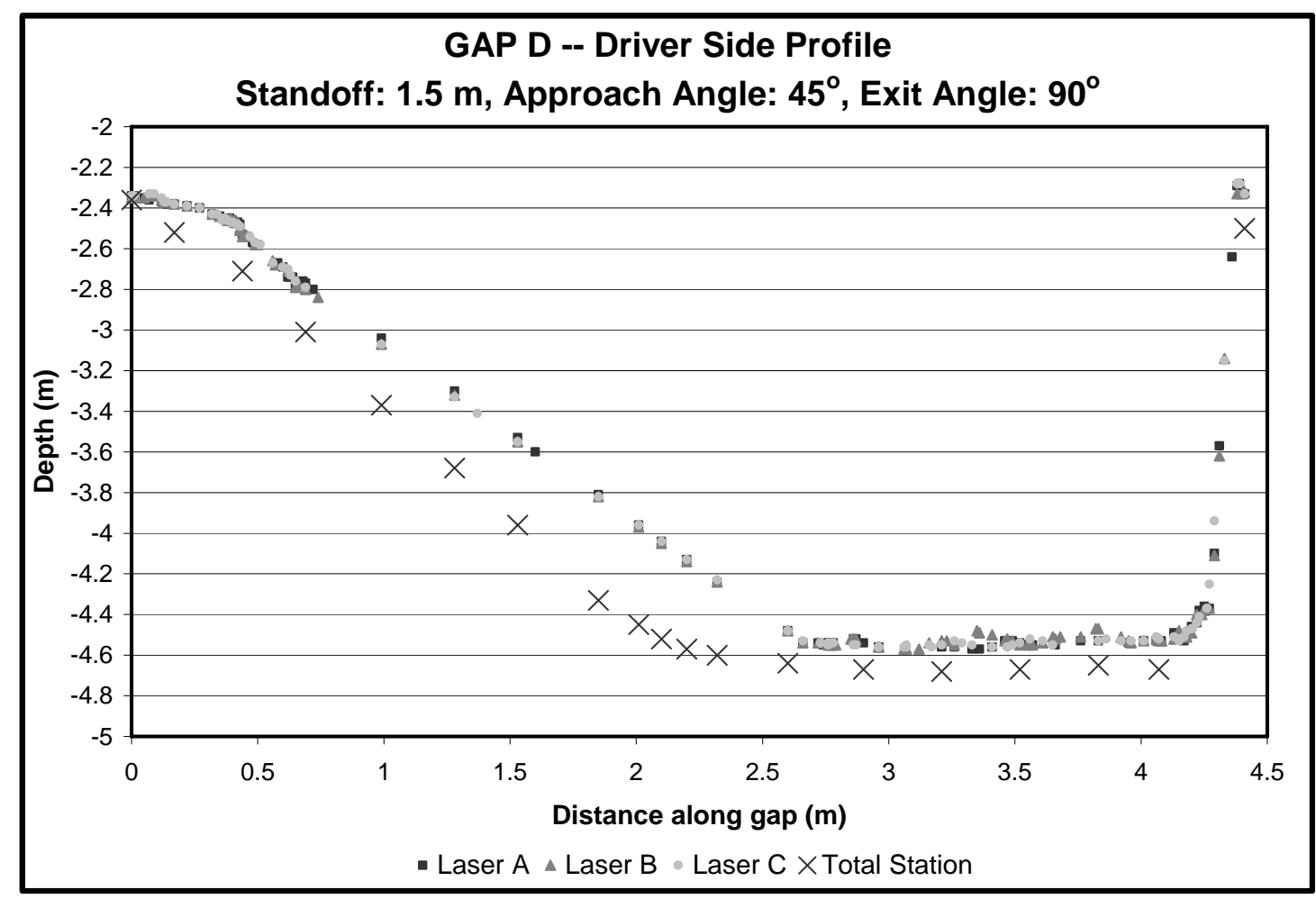

Figure 82. Data for Gap D - driver side profile.

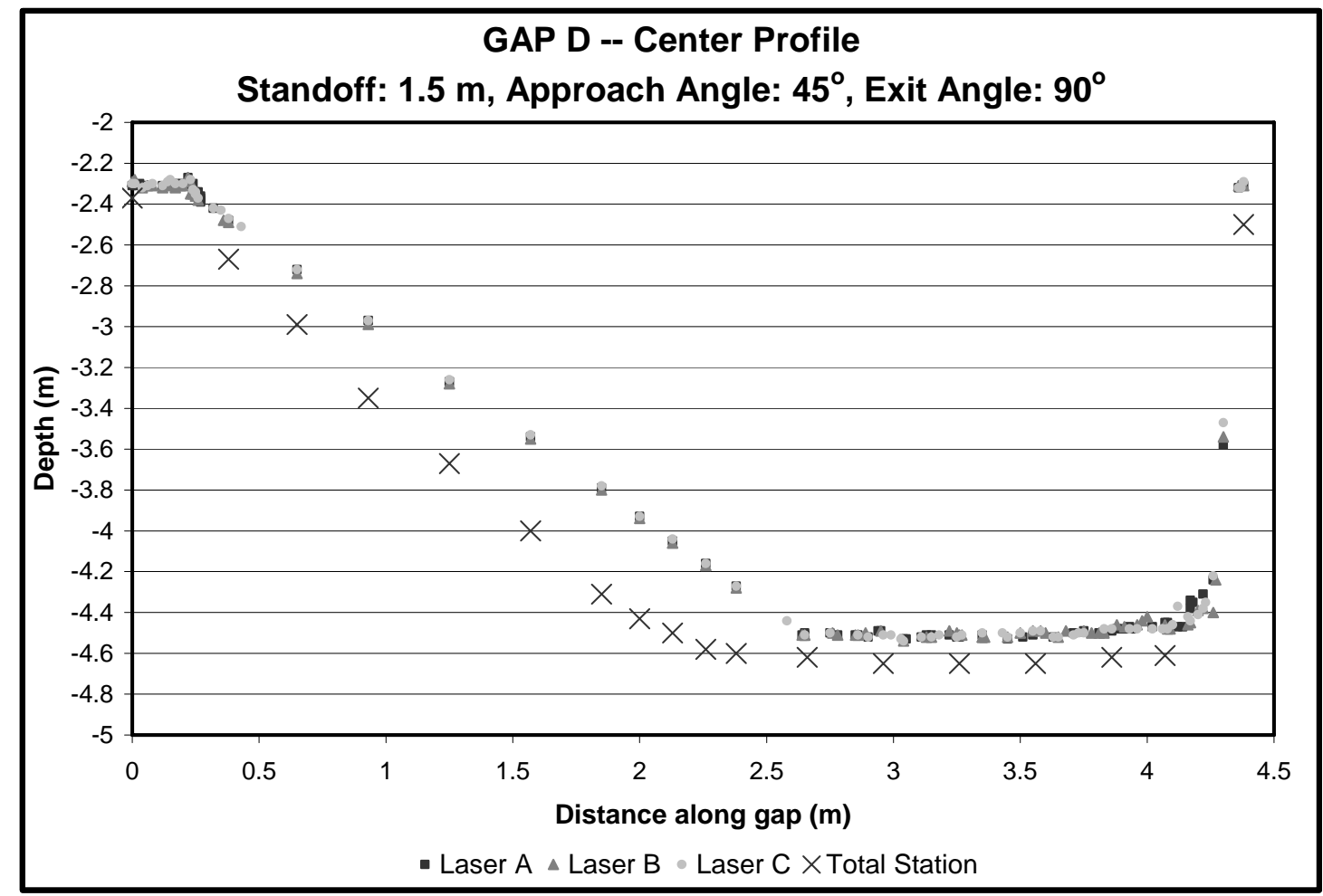

Figure 83. Data for Gap D - center profile. 


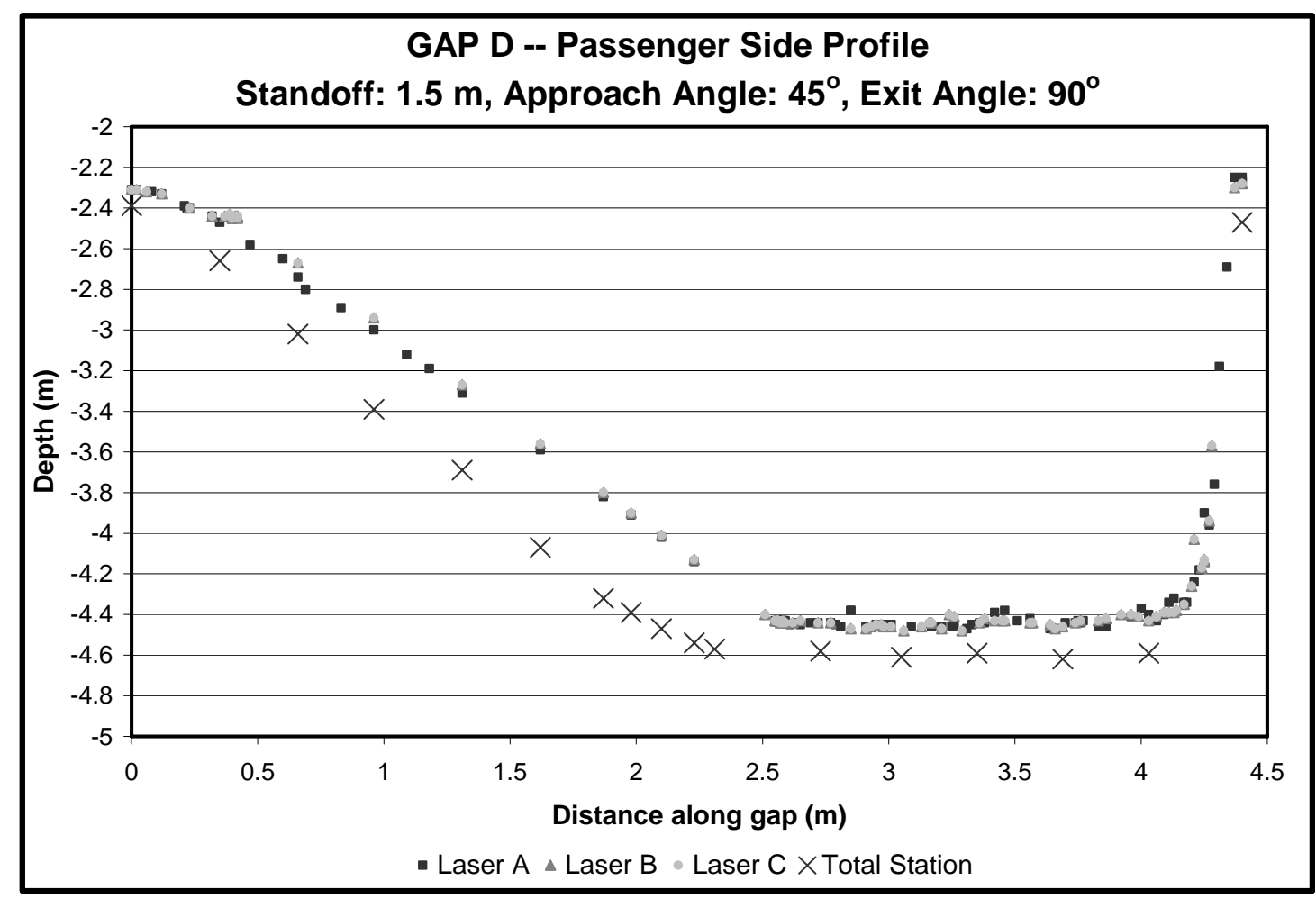

Figure 84. Data for Gap D - passenger side profile.

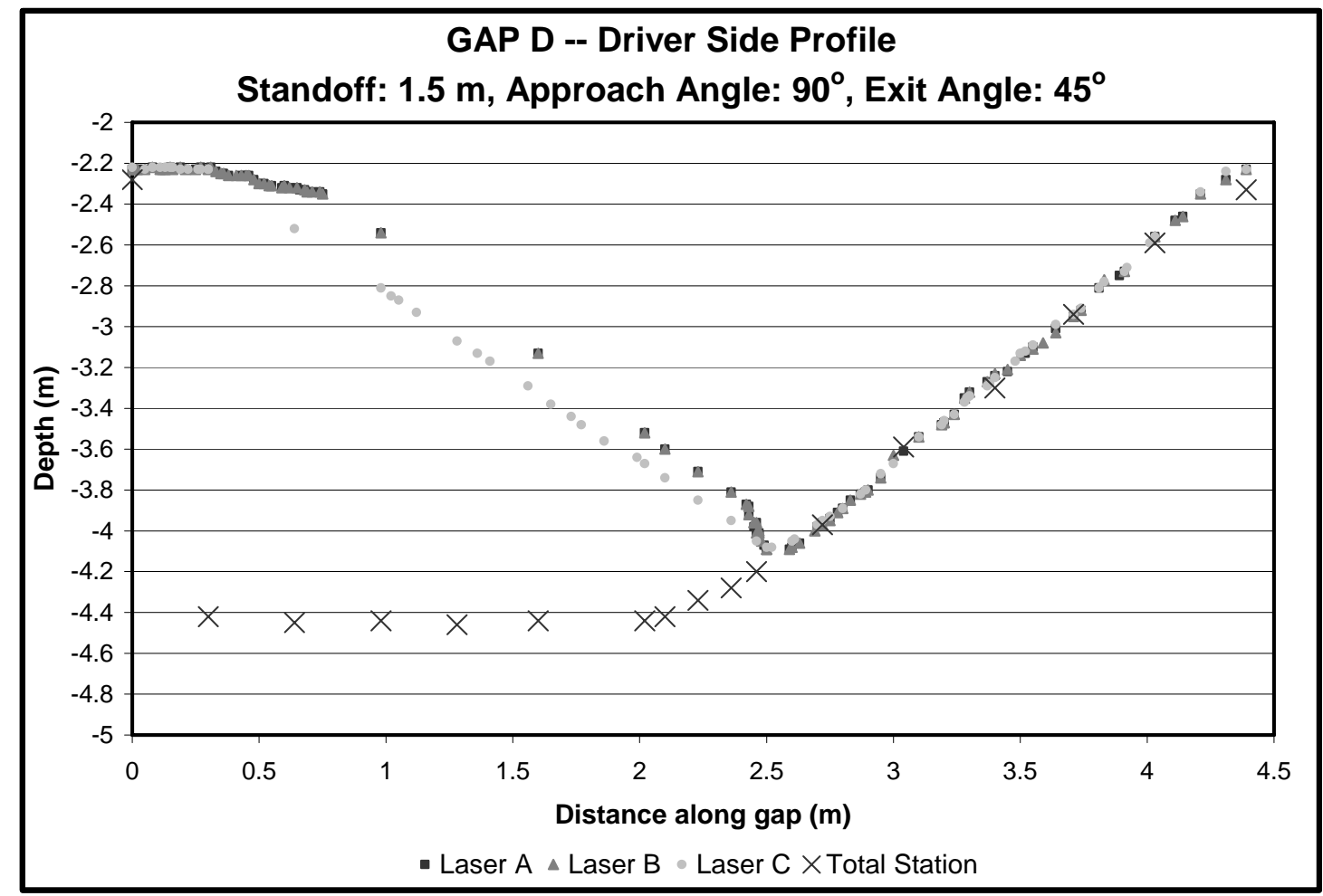

Figure 85. Data for Gap D - driver side profile. 


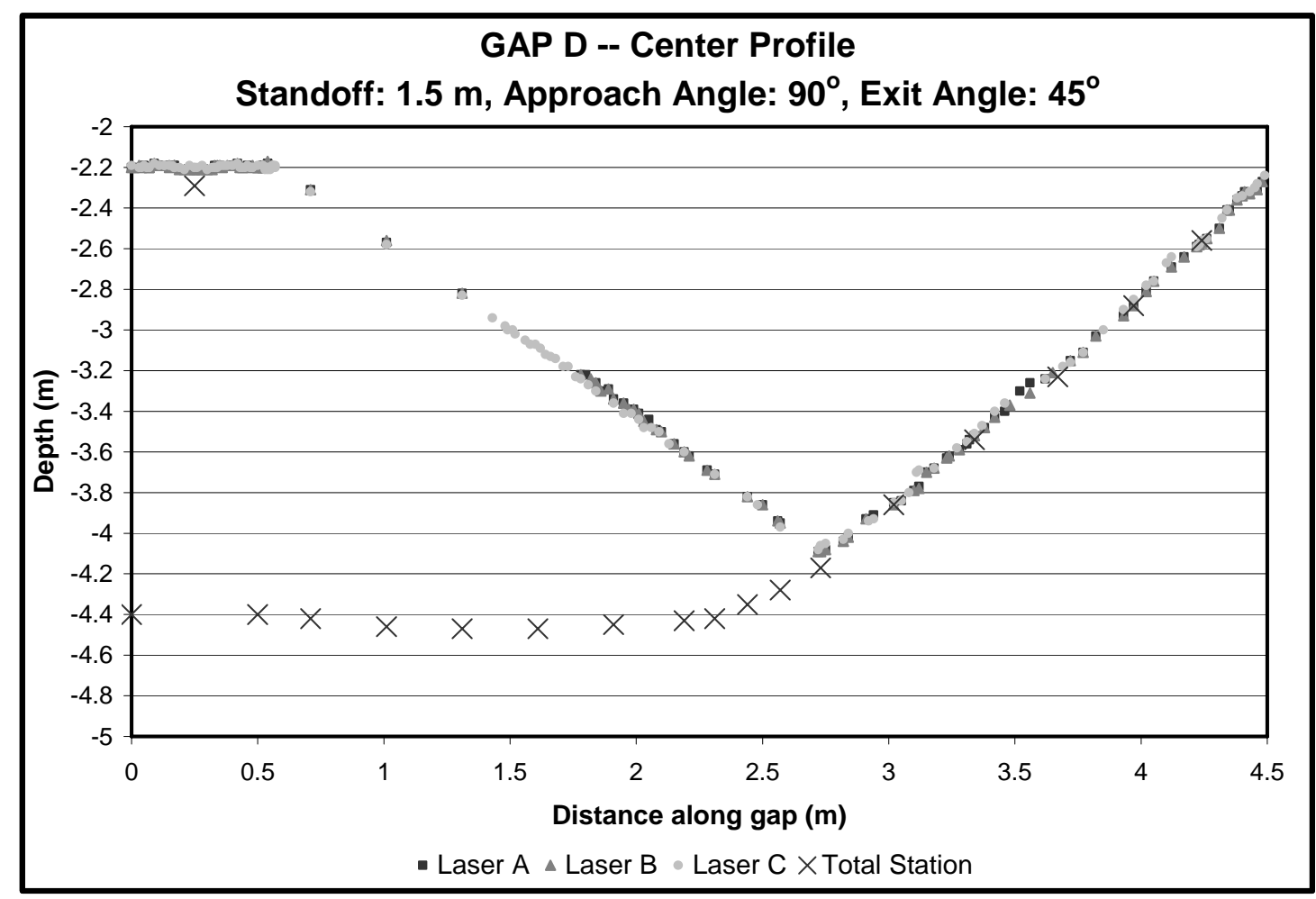

Figure 86. Data for Gap D - center profile.

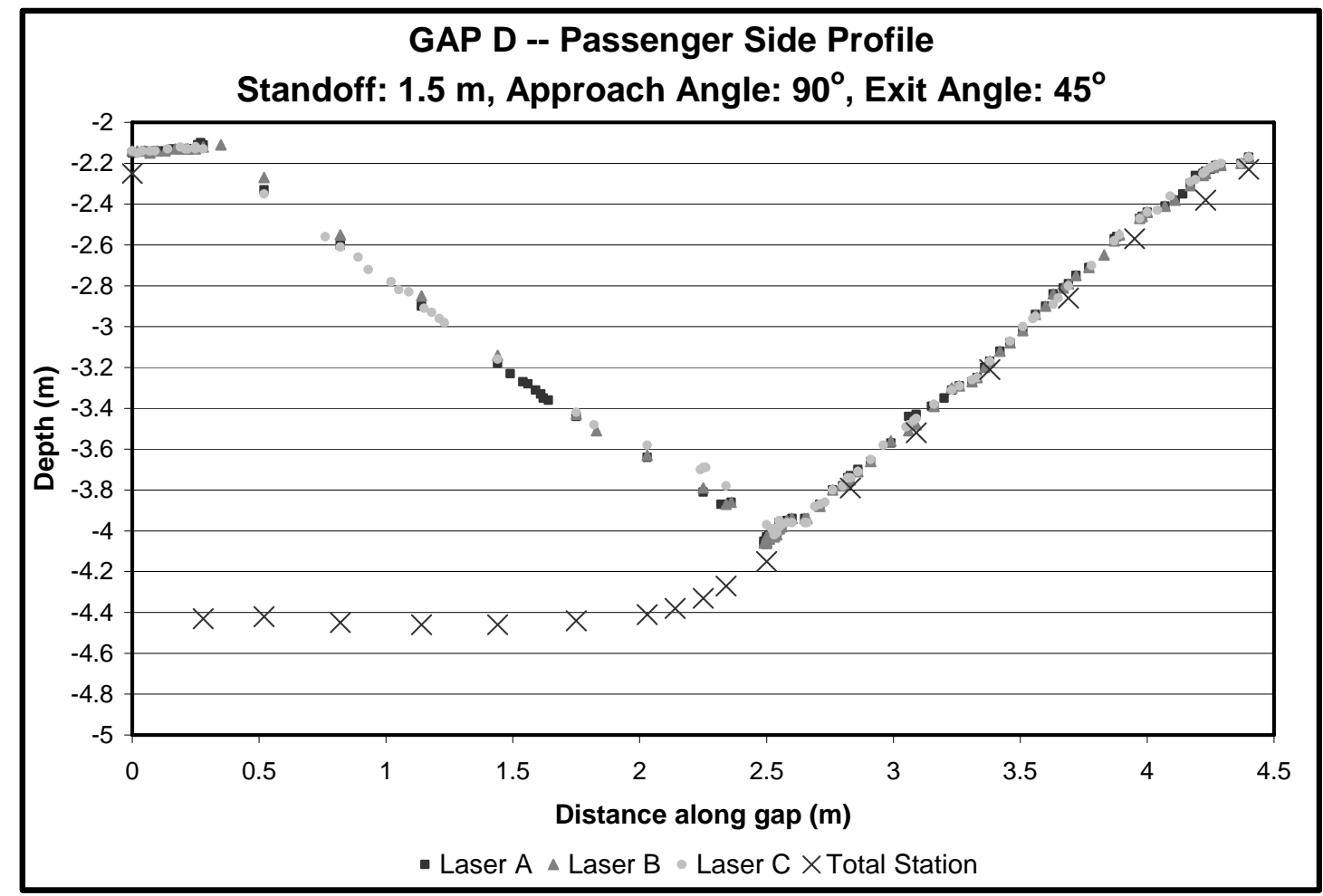

Figure 87. Data for Gap D - passenger side profile. 


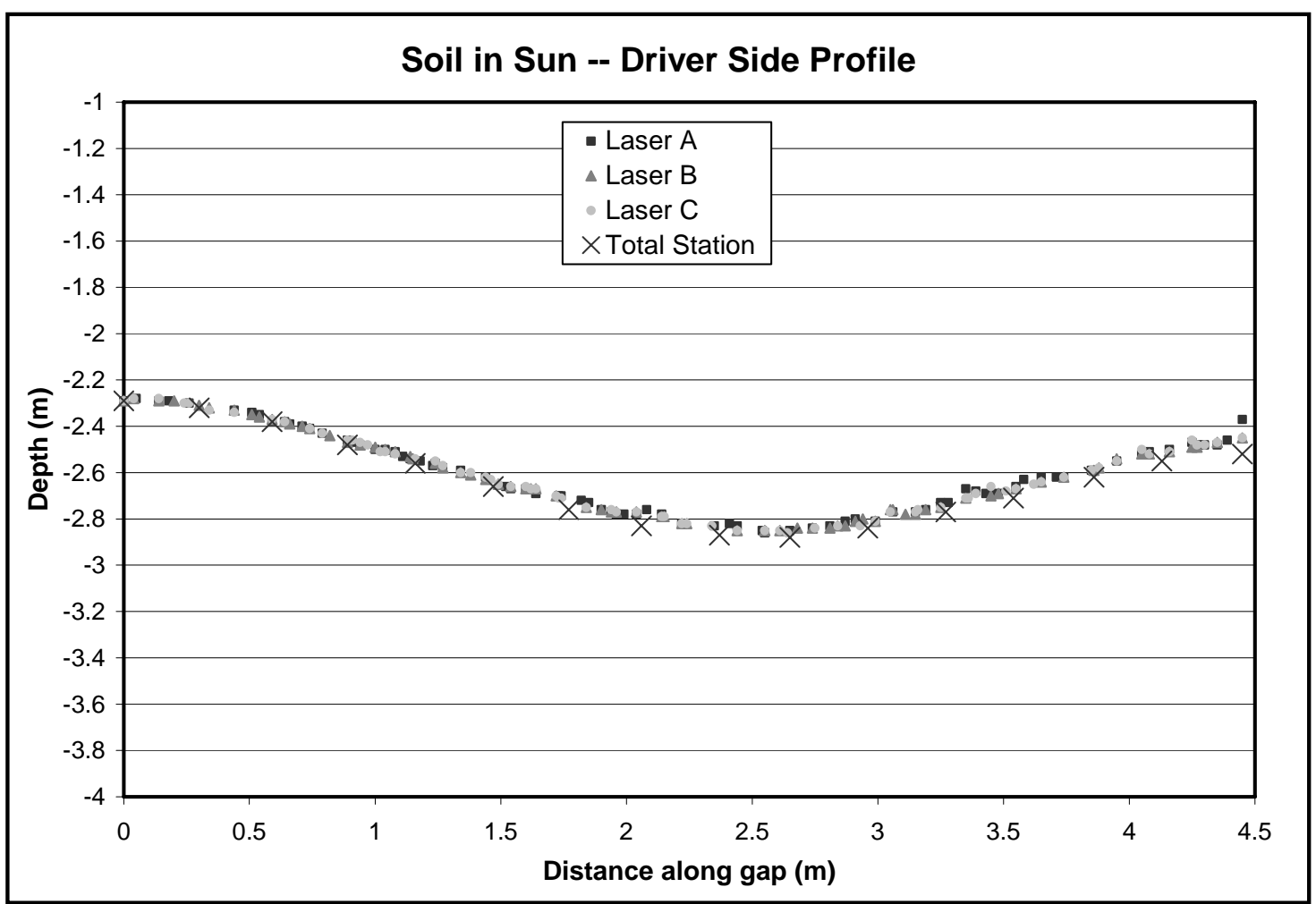

Figure 88. Data for soil gap taken in sunlight - driver side profile.

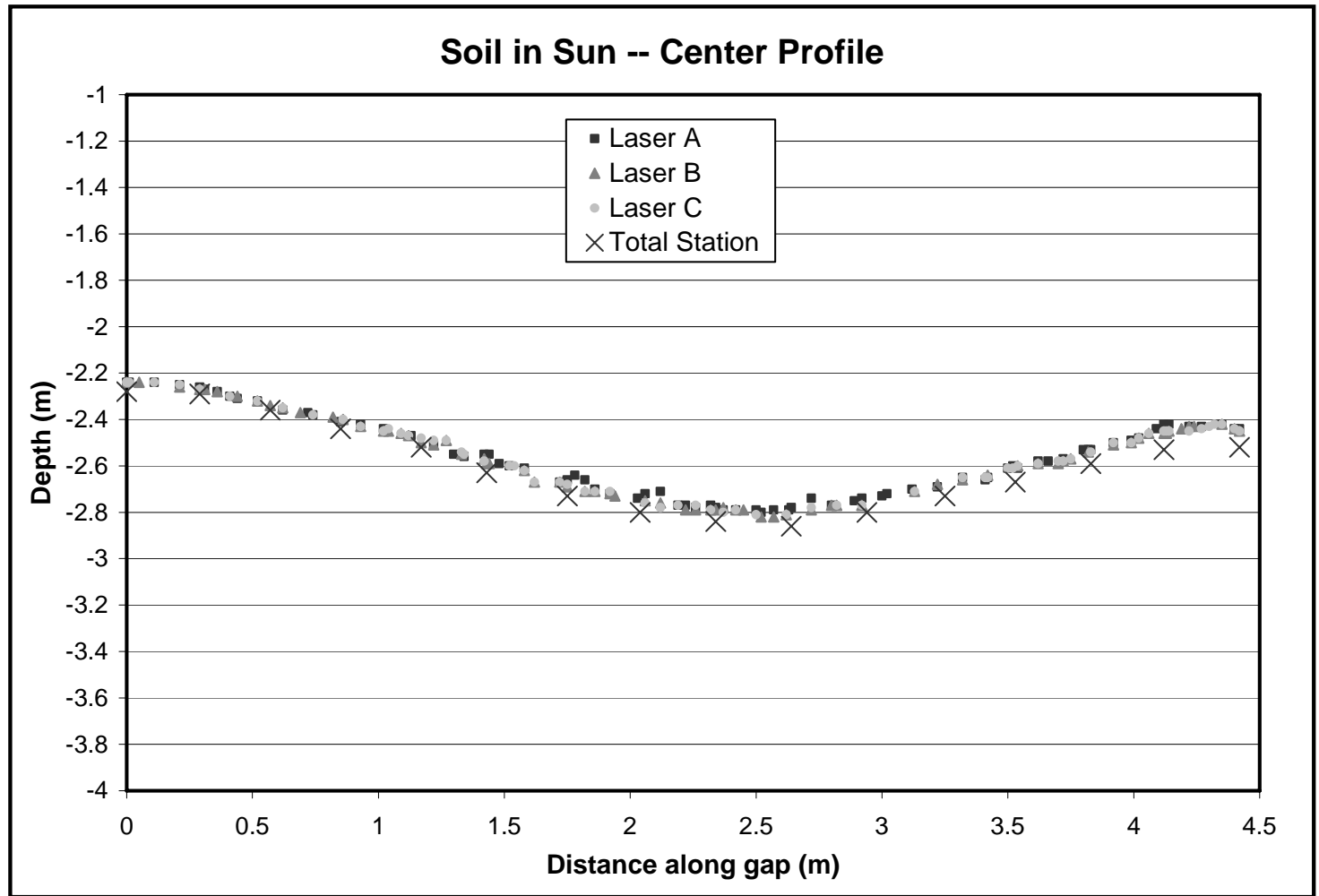

Figure 89. Data for soil gap taken in sunlight - center profile. 


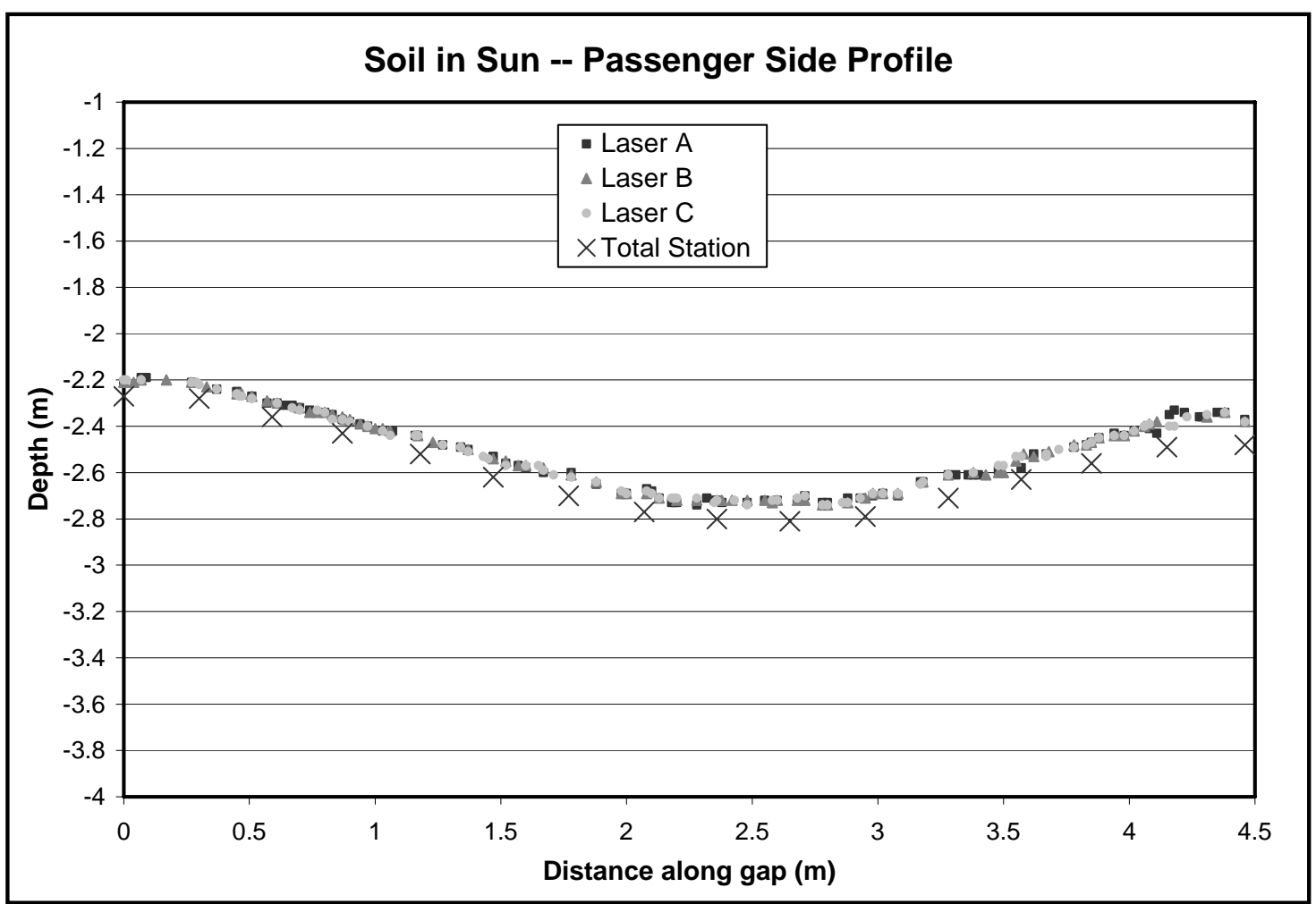

Figure 90. Data for soil gap taken in sunlight - passenger side profile.

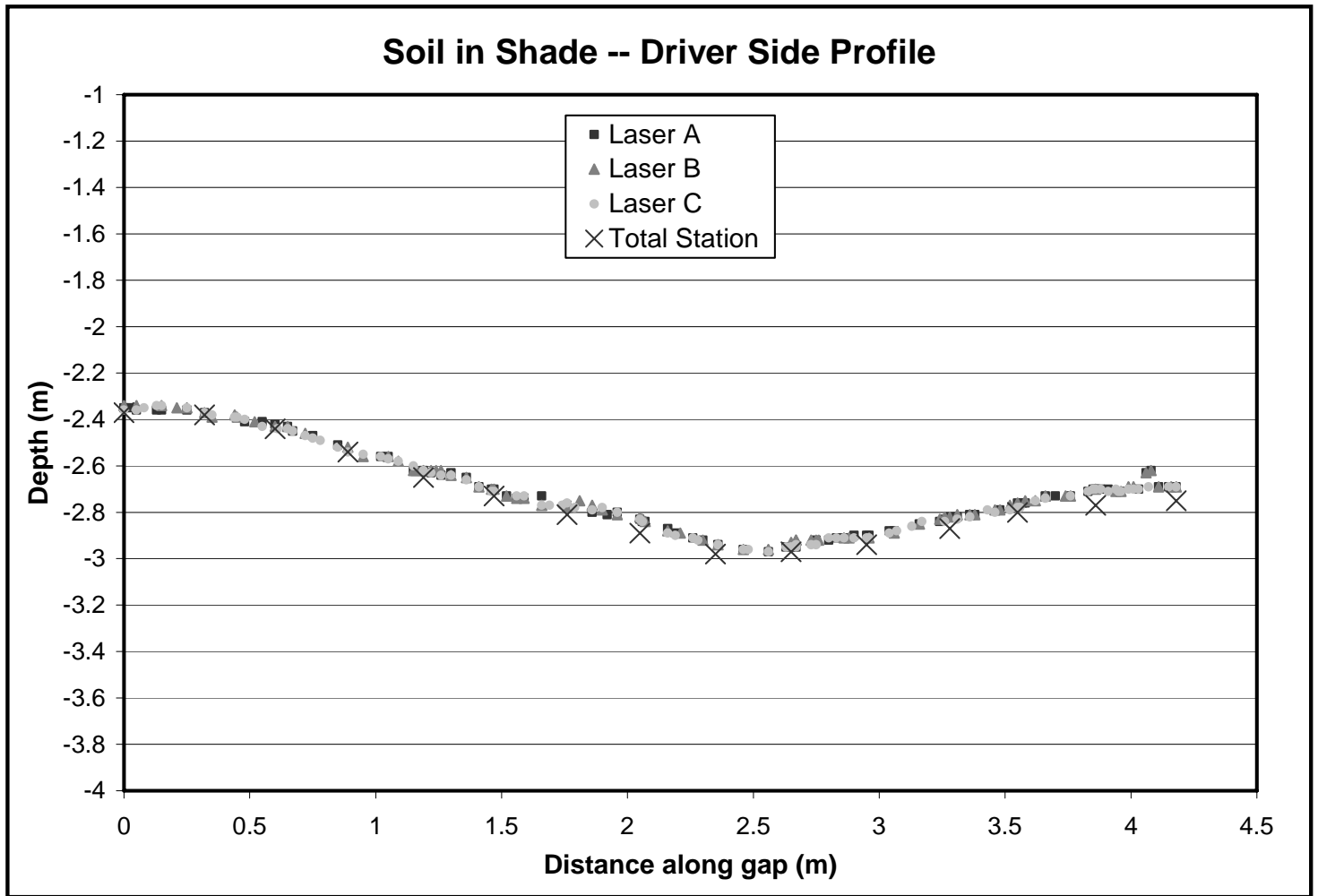

Figure 91. Data for soil gap taken in the shade - driver side profile. 


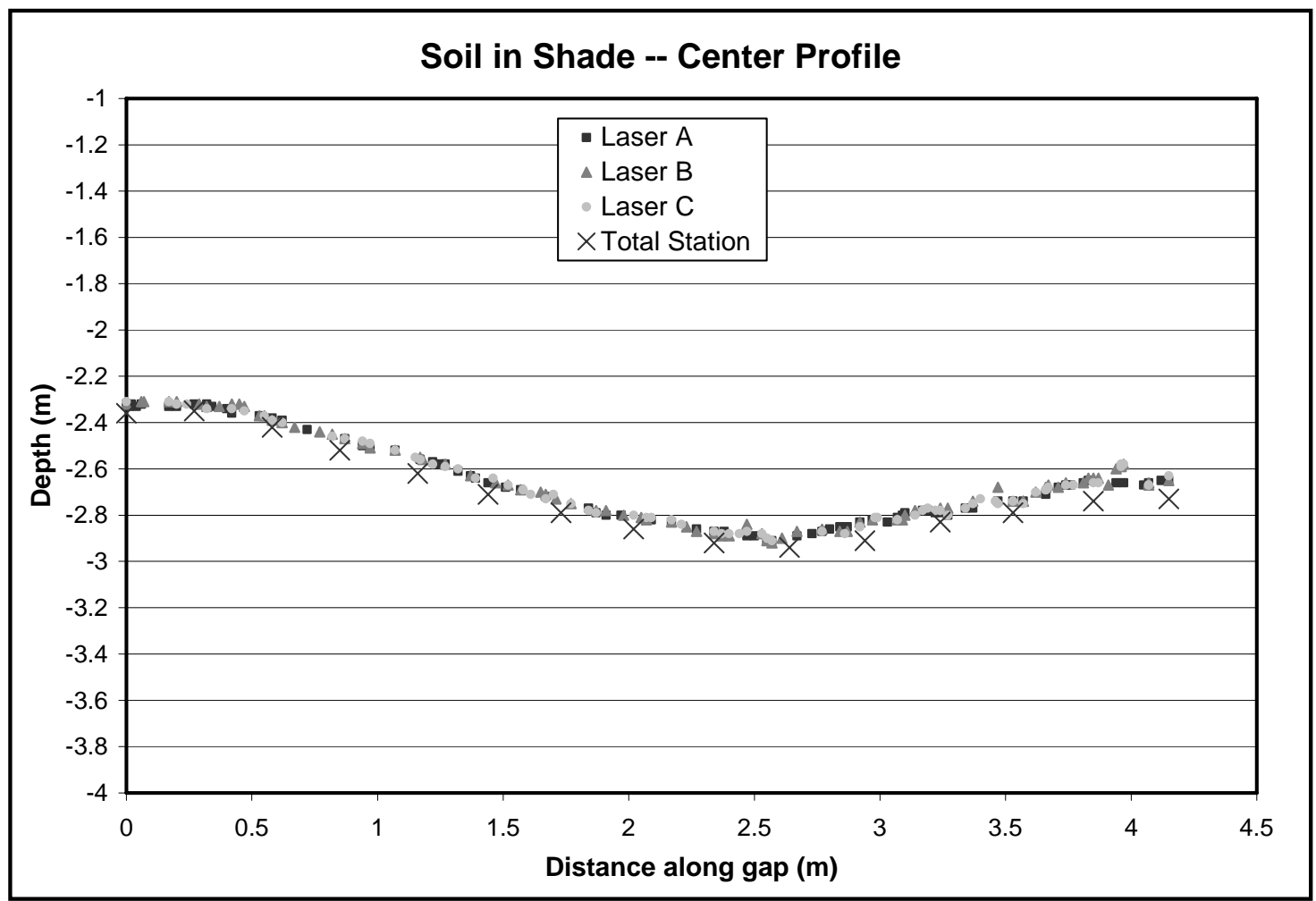

Figure 92. Data for soil gap taken in the shade - center profile.

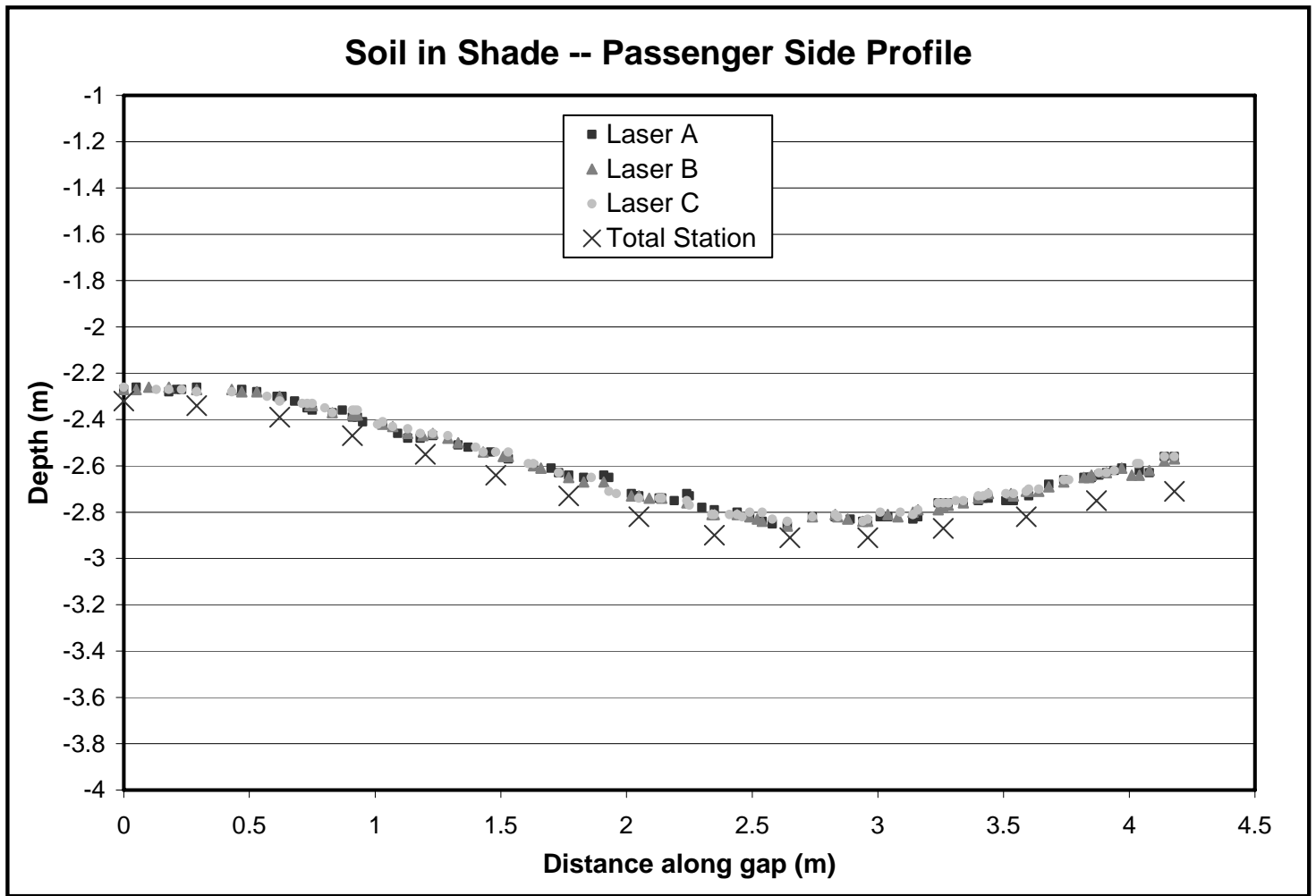

Figure 93. Data for soil gap taken in the shade - passenger side profile. 


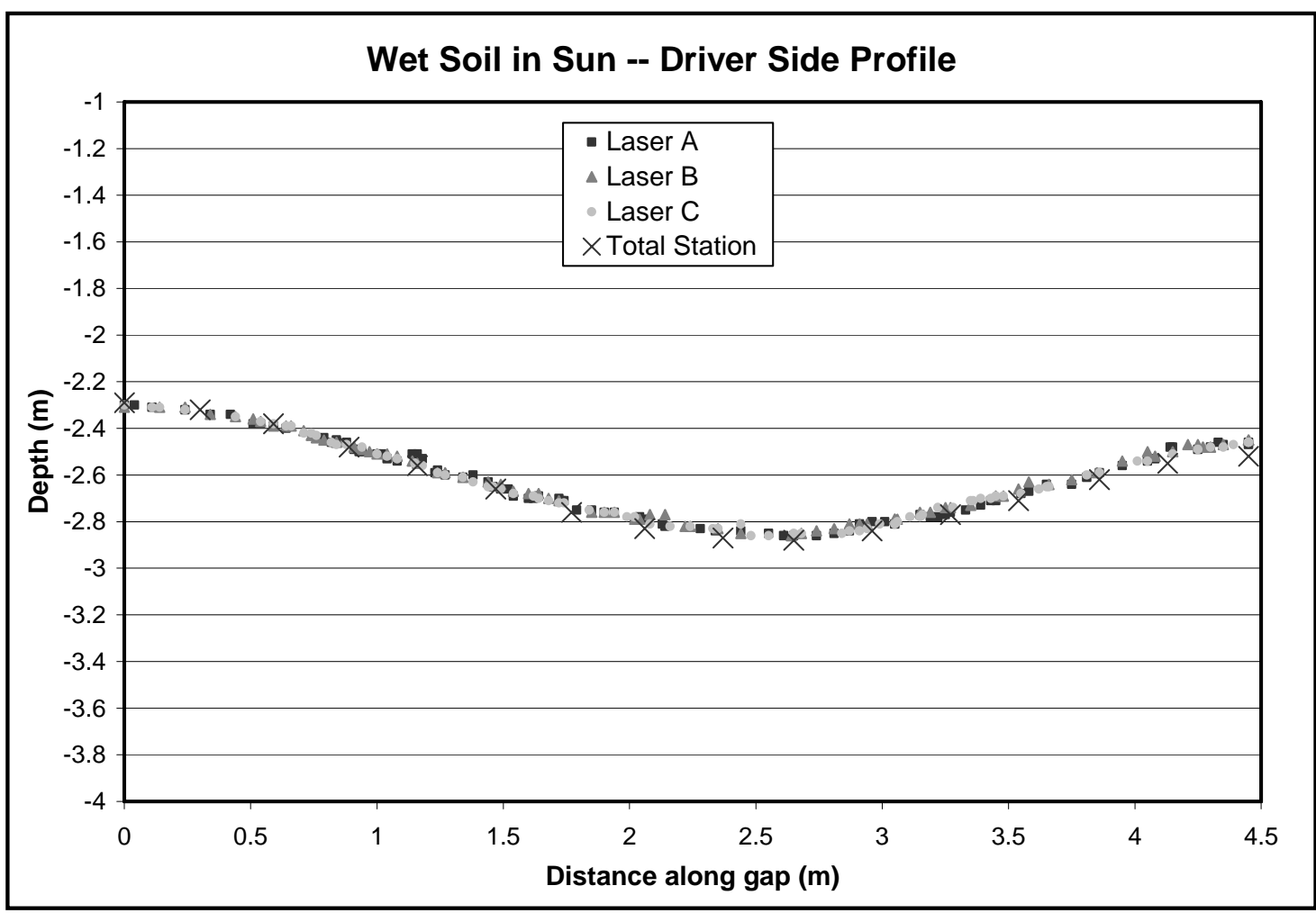

Figure 94. Data for wet soil gap taken in the sun - driver side profile.

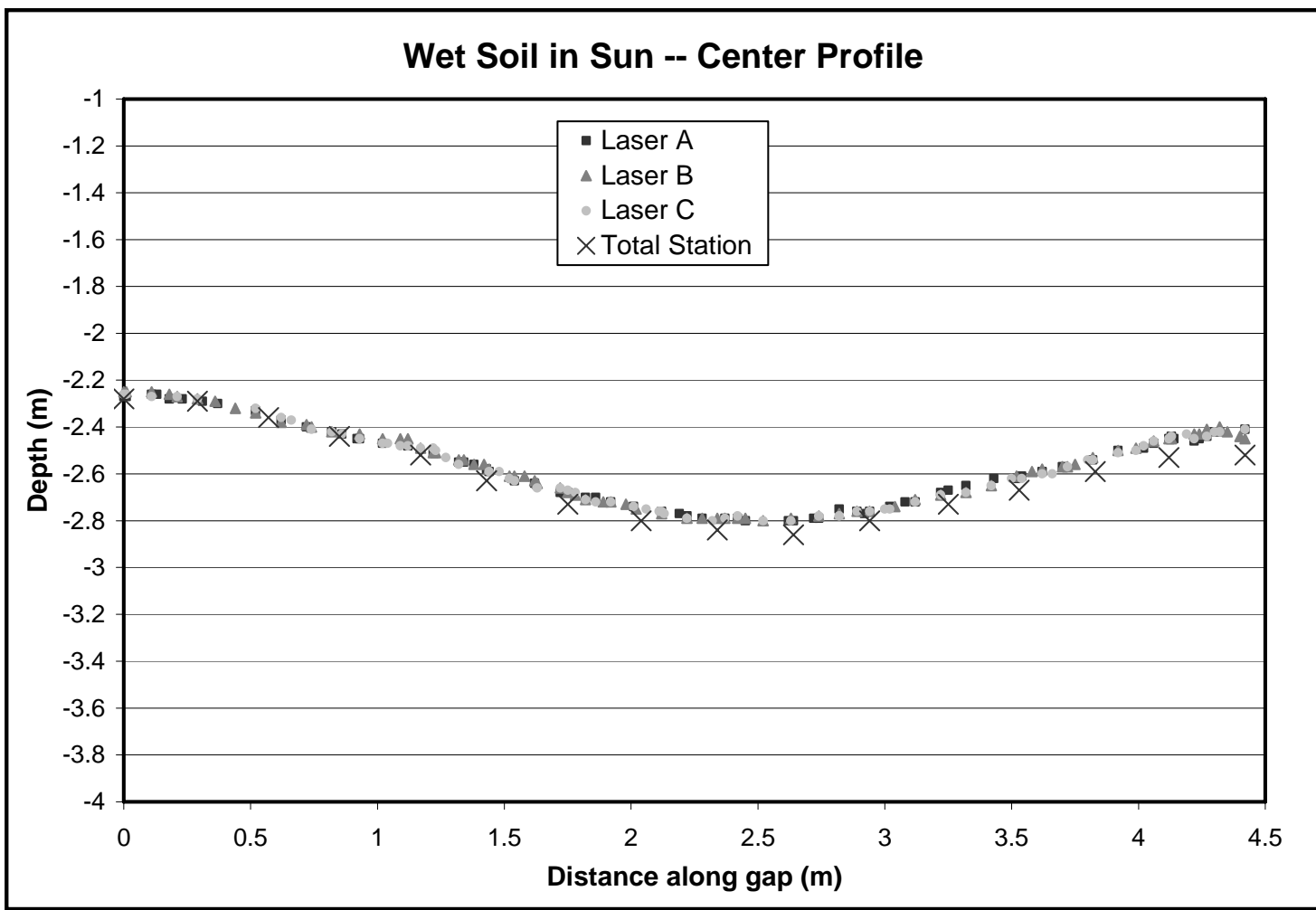

Figure 95. Data for wet soil gap taken in the sun - center profile. 


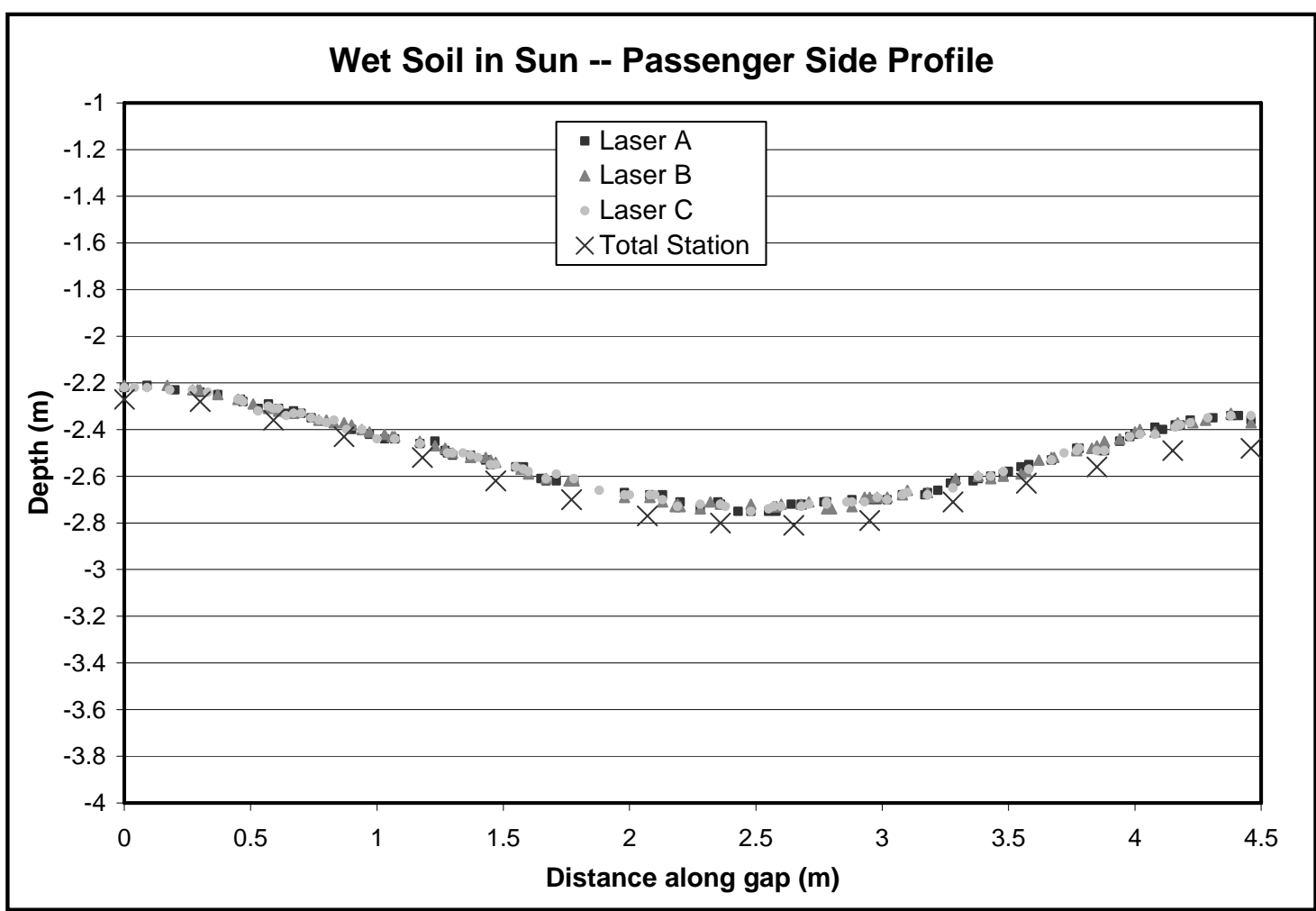

Figure 96. Data for wet soil gap taken in the sun - passenger side profile.

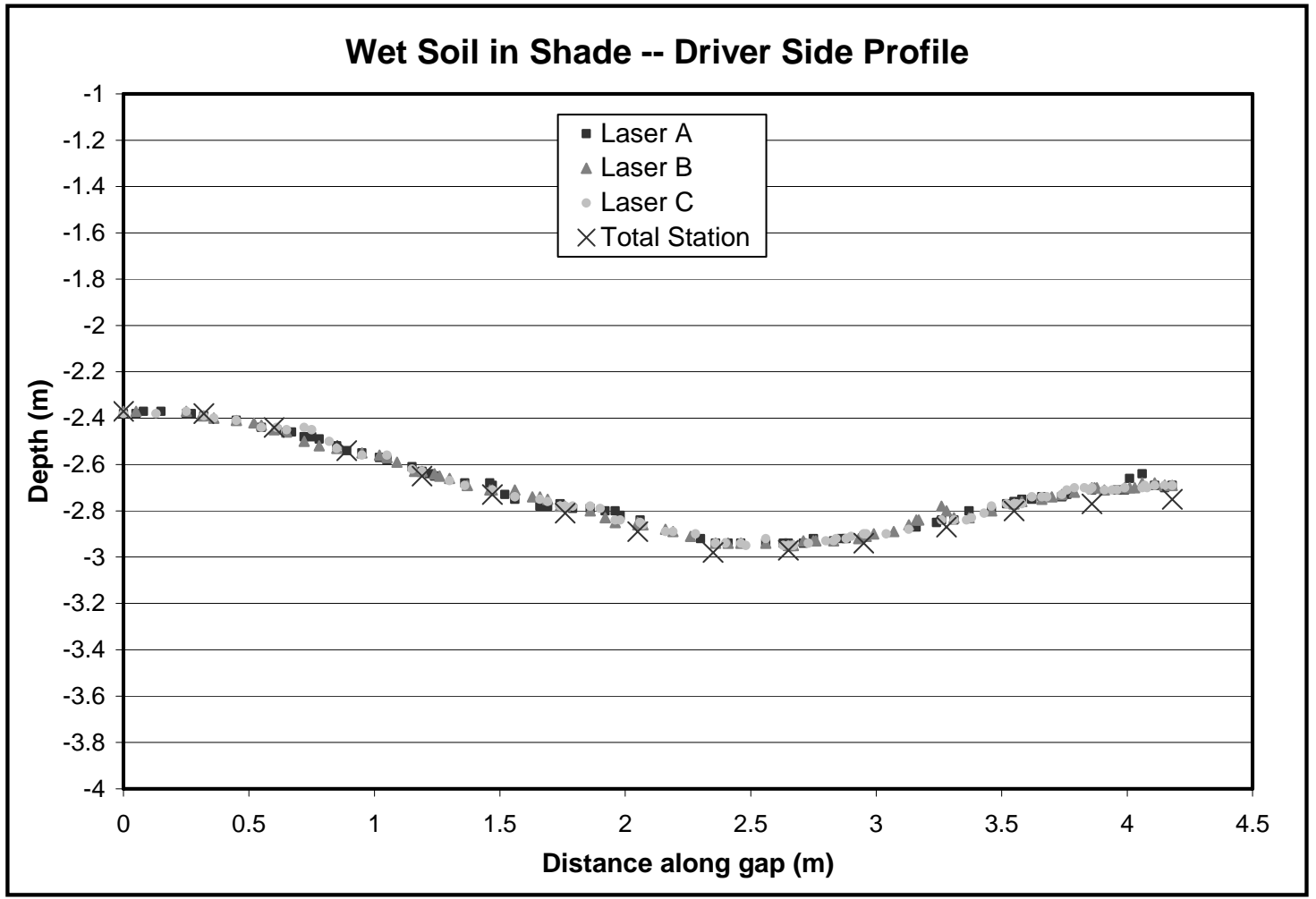

Figure 97. Data for wet soil gap taken in the shade - driver side profile. 


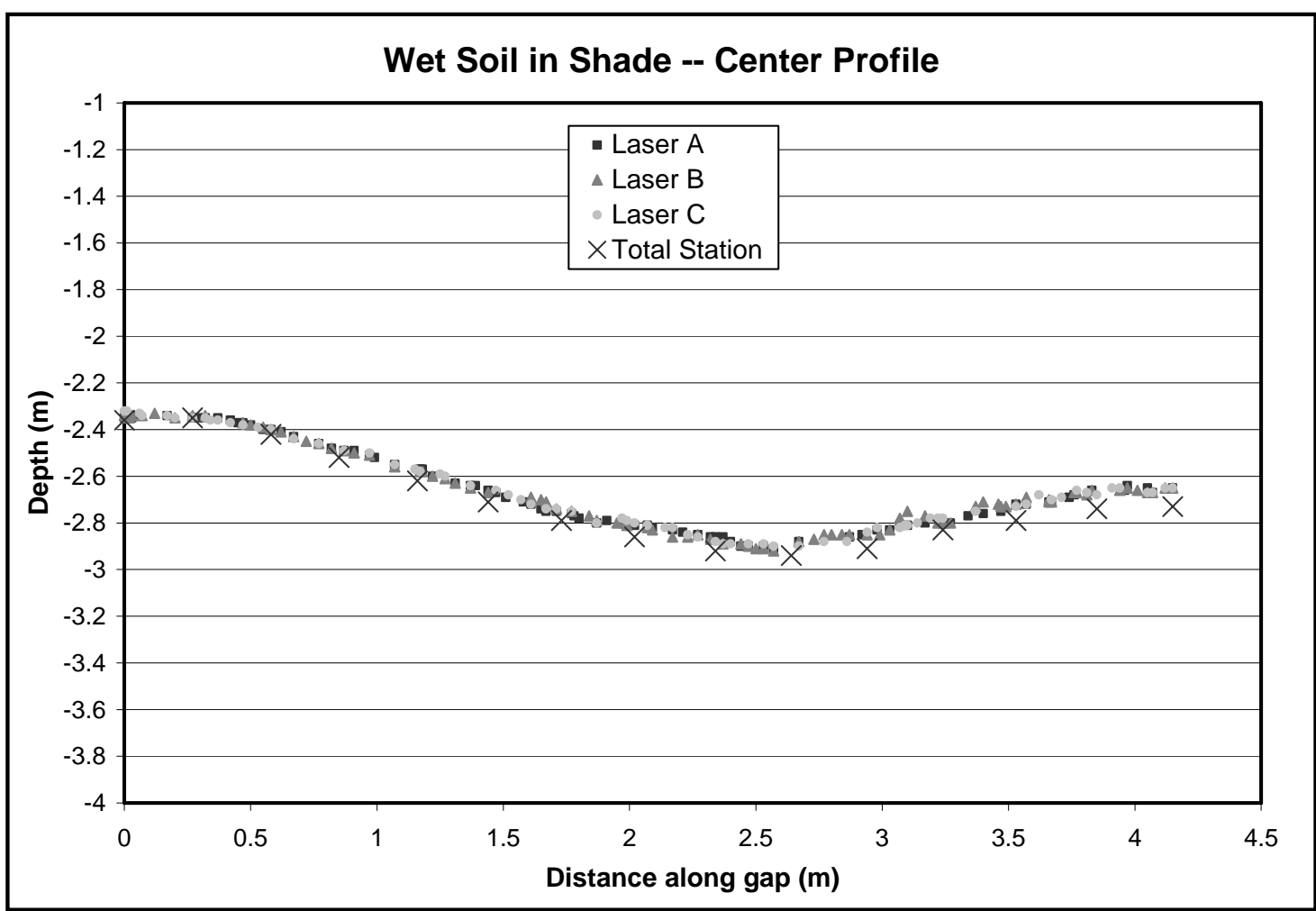

Figure 98. Data for wet soil gap taken in the shade - center profile.

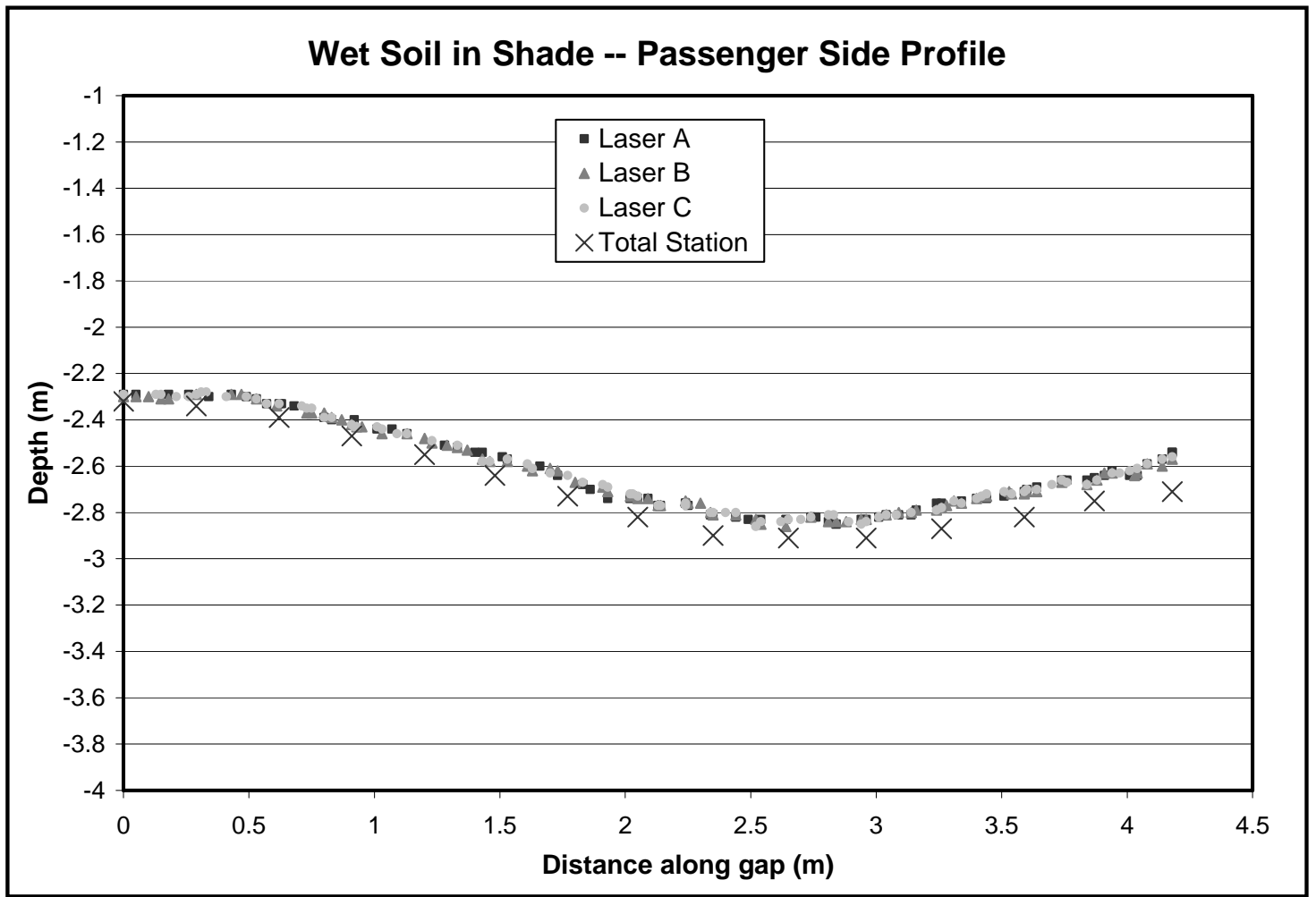

Figure 99. Data for wet soil gap taken in the shade - passenger side profile. 


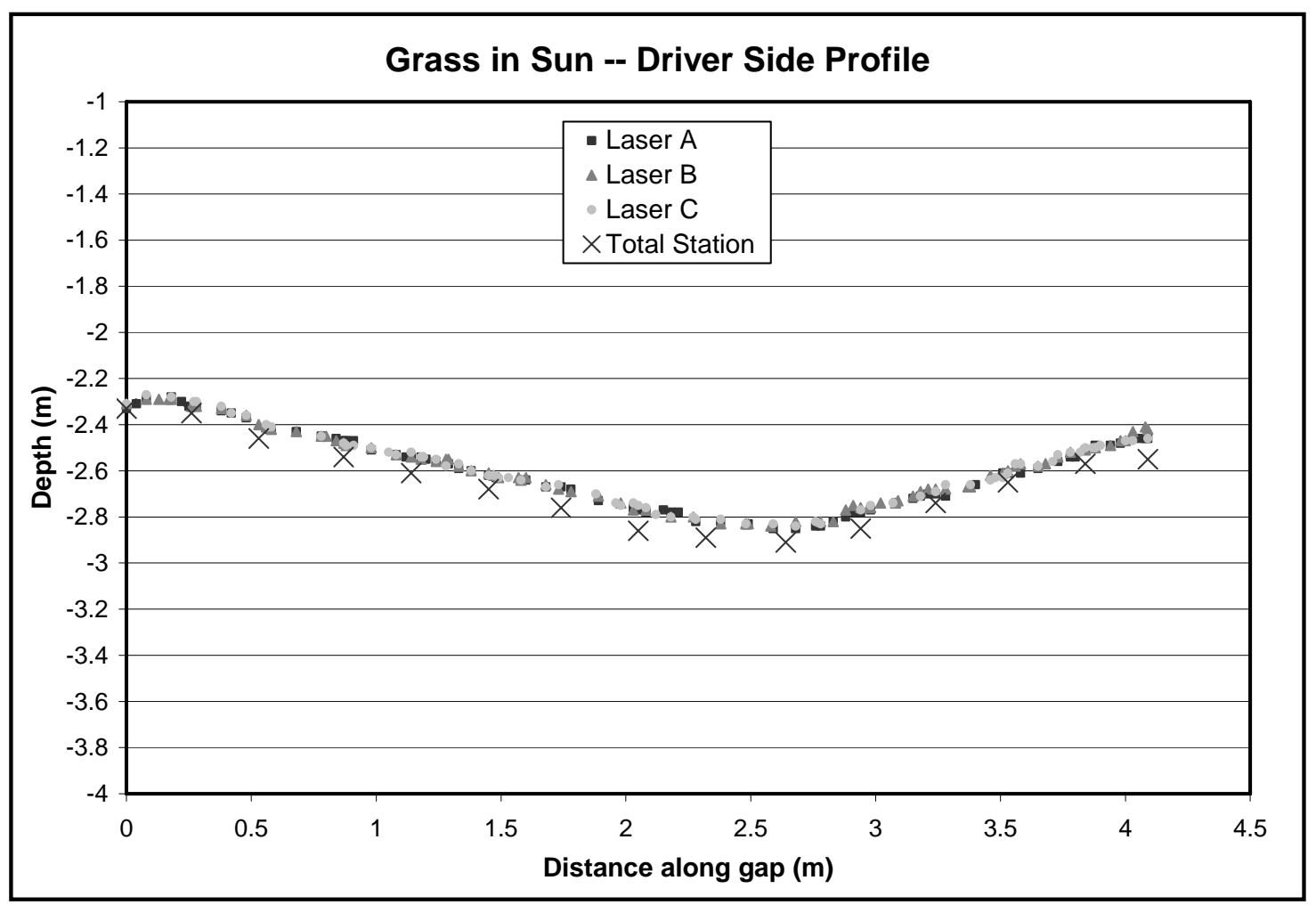

Figure 100. Data for grass gap taken in the sun - driver side profile.

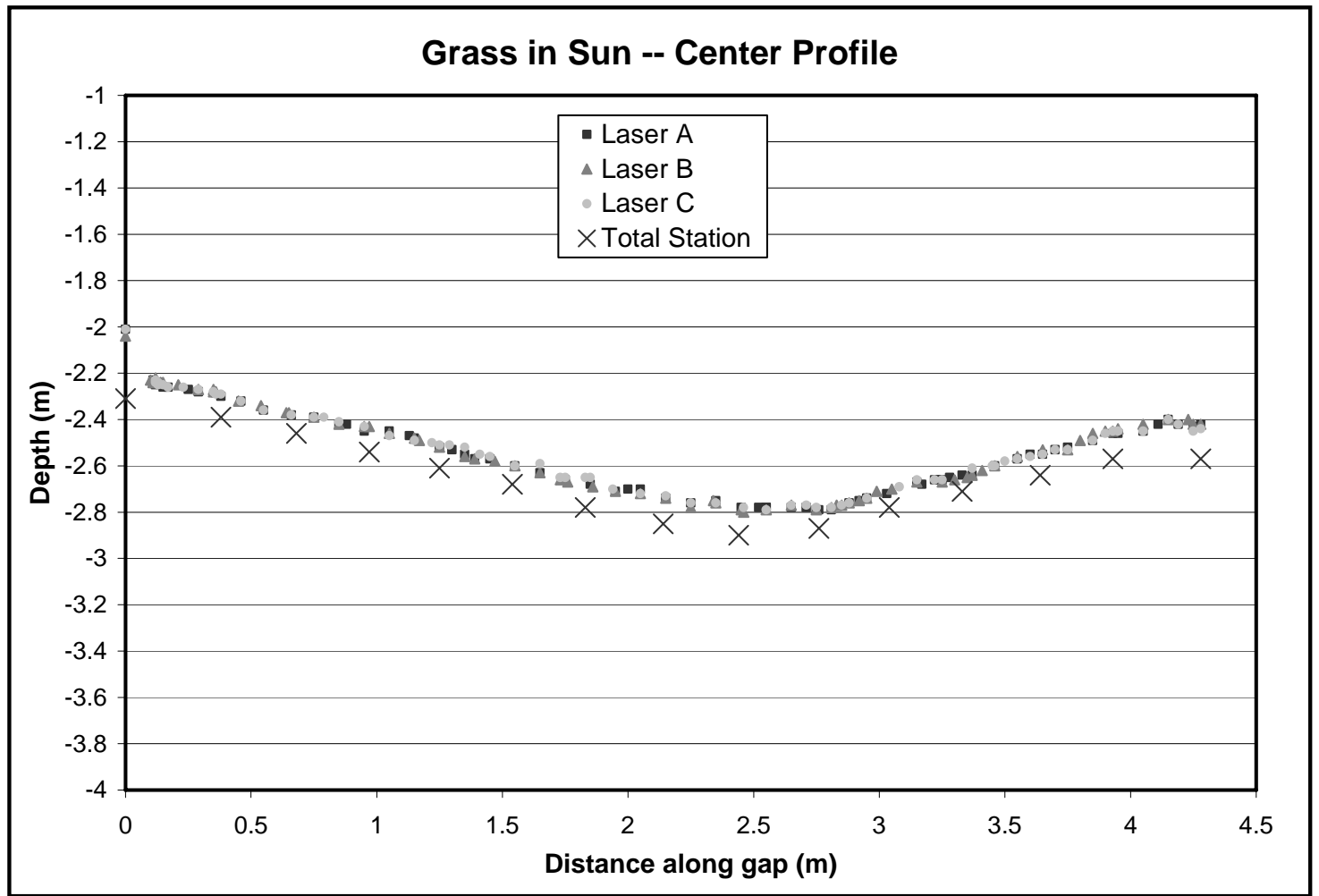

Figure 101. Data for grass gap taken in the sun - center profile. 


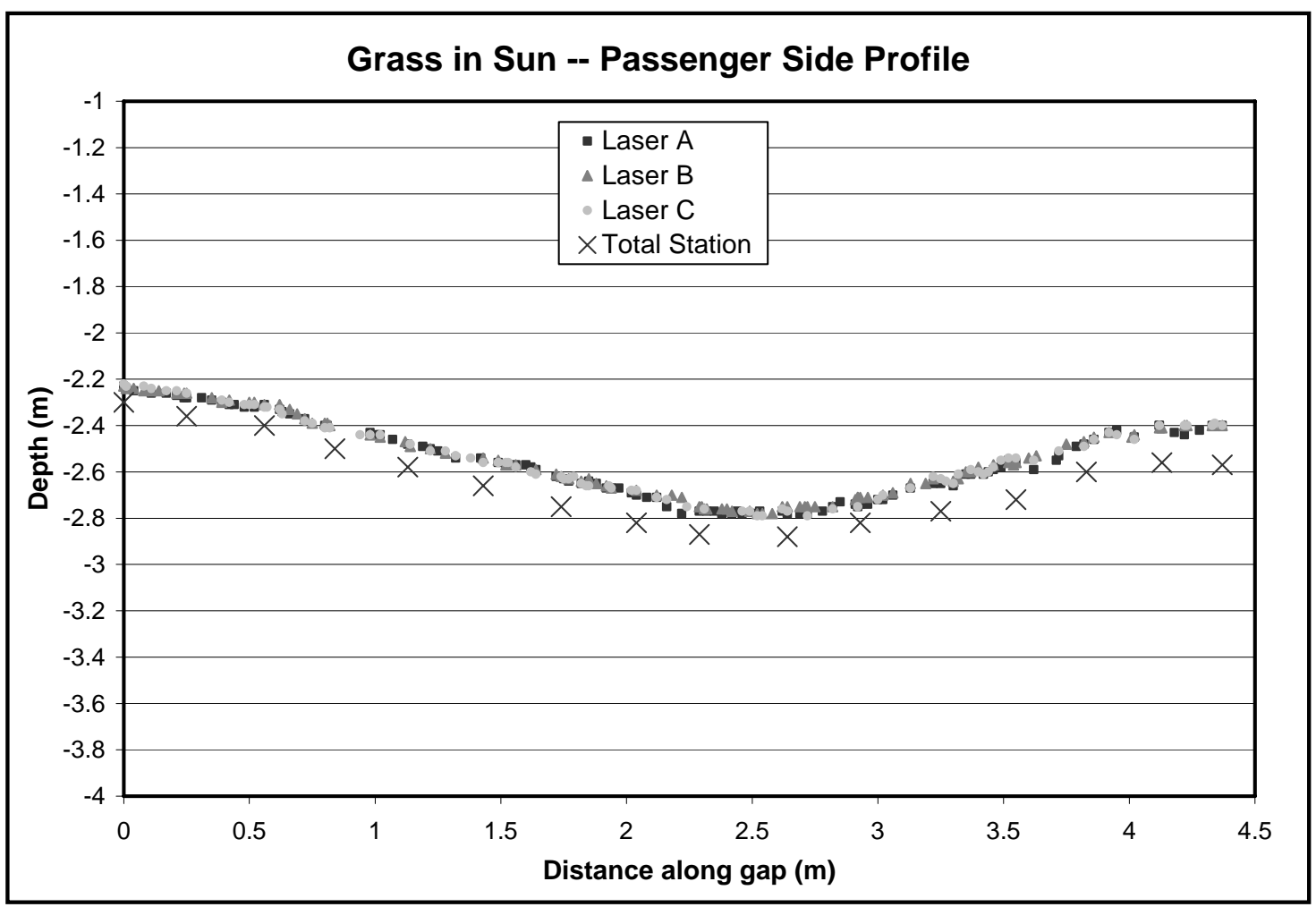

Figure 102. Data for grass gap taken in the sun - passenger side profile.

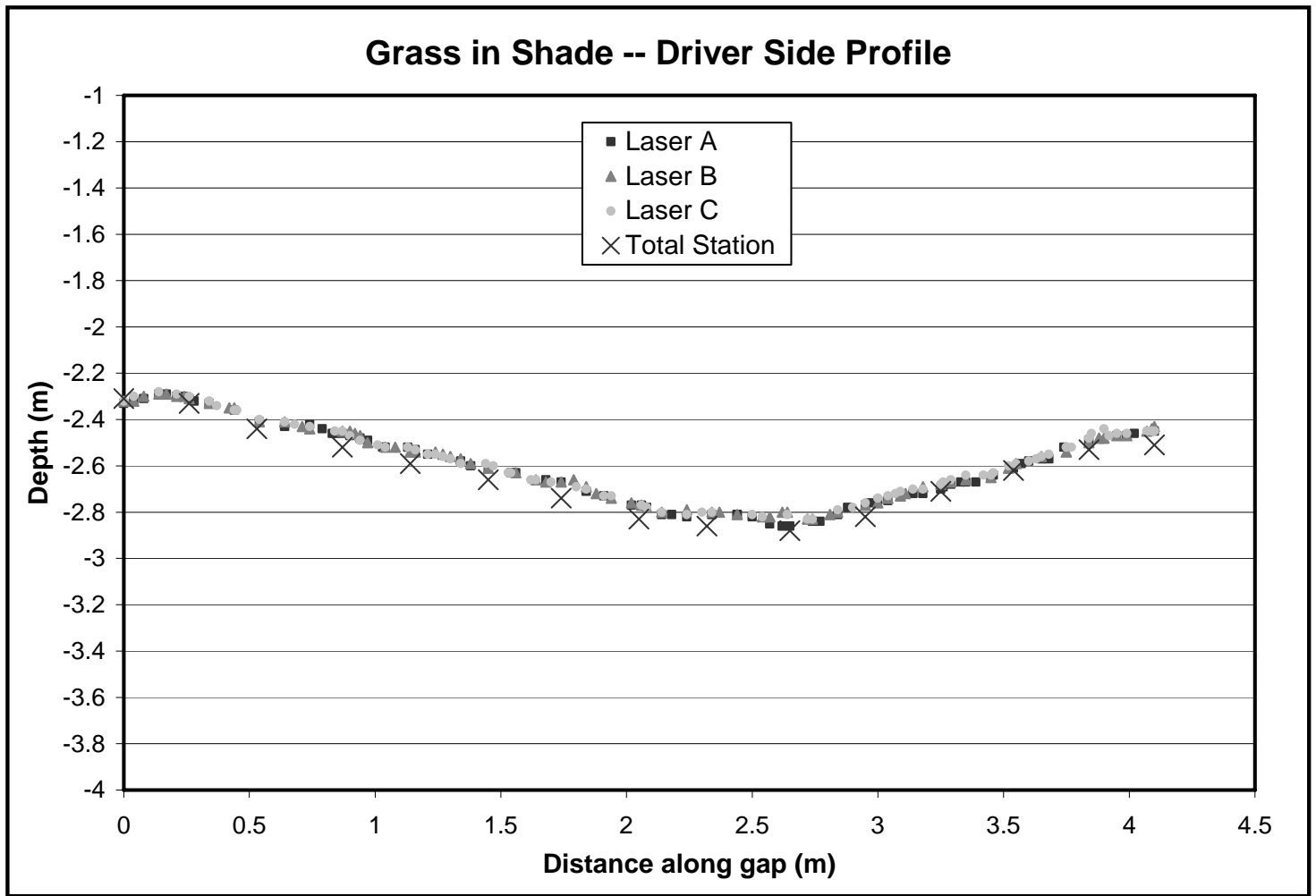

Figure 103. Data for grass gap taken in the shade - driver side profile. 


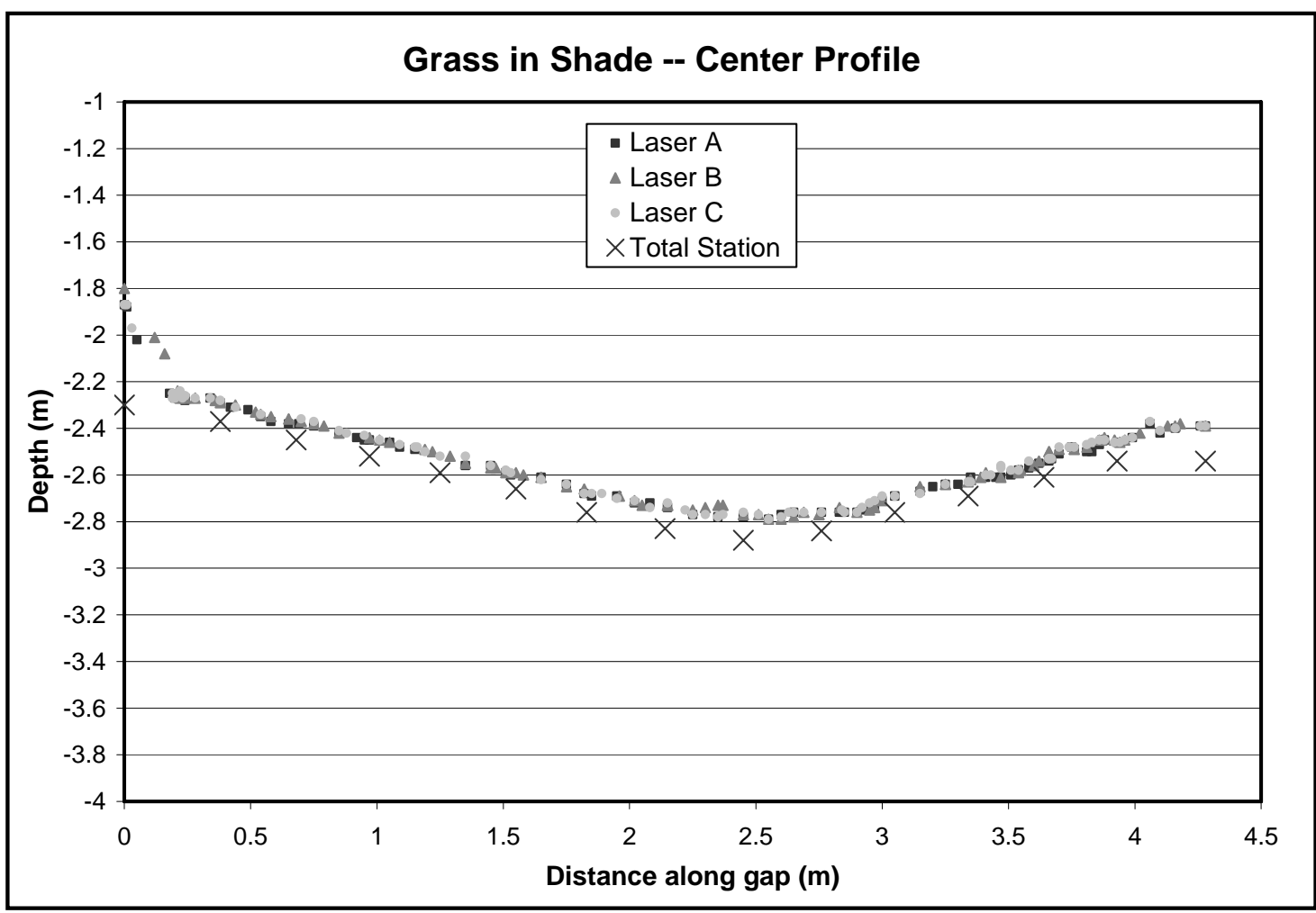

Figure 104. Data for grass gap taken in the shade - center profile.

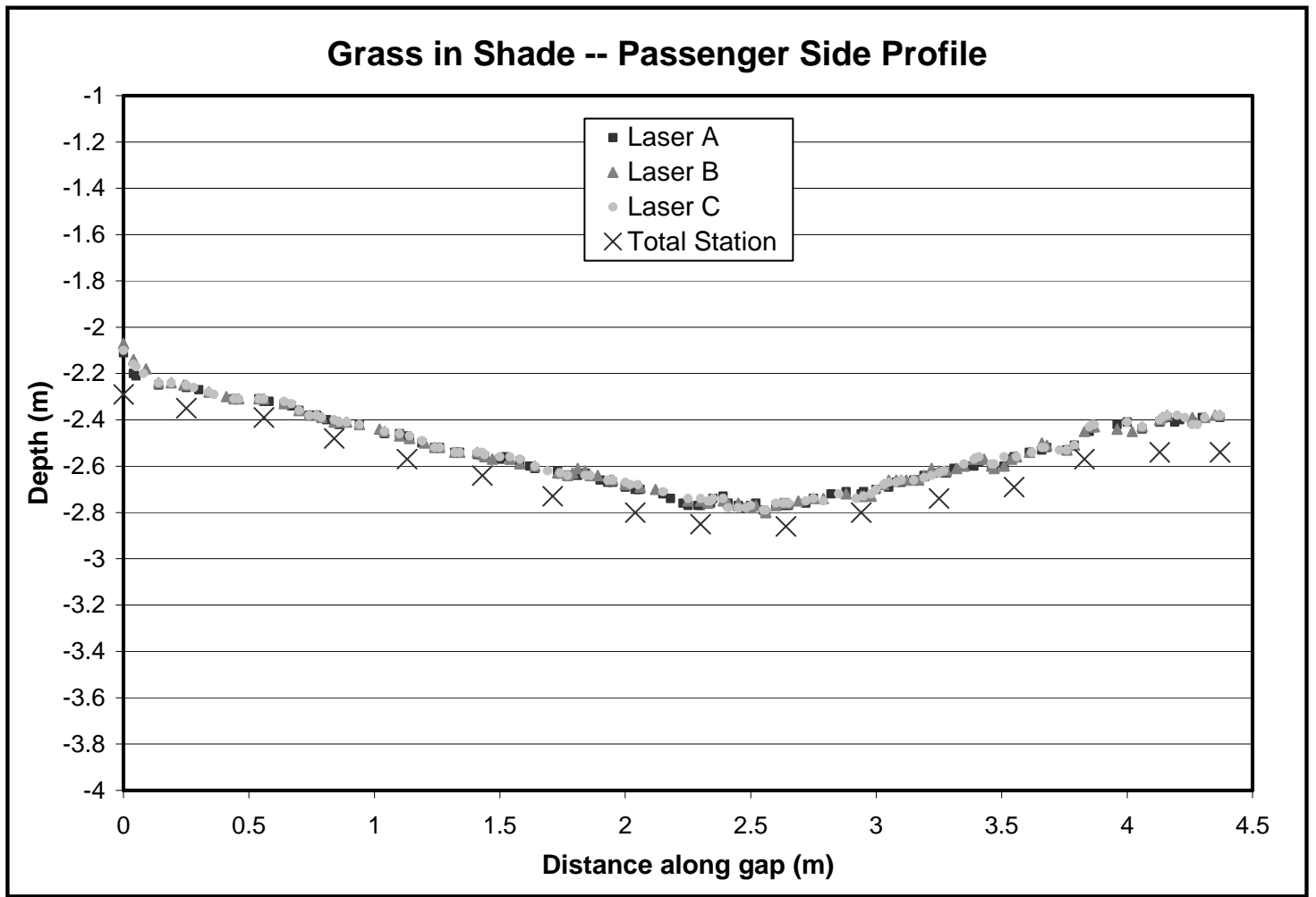

Figure 105. Data for grass gap taken in the shade - passenger side profile. 


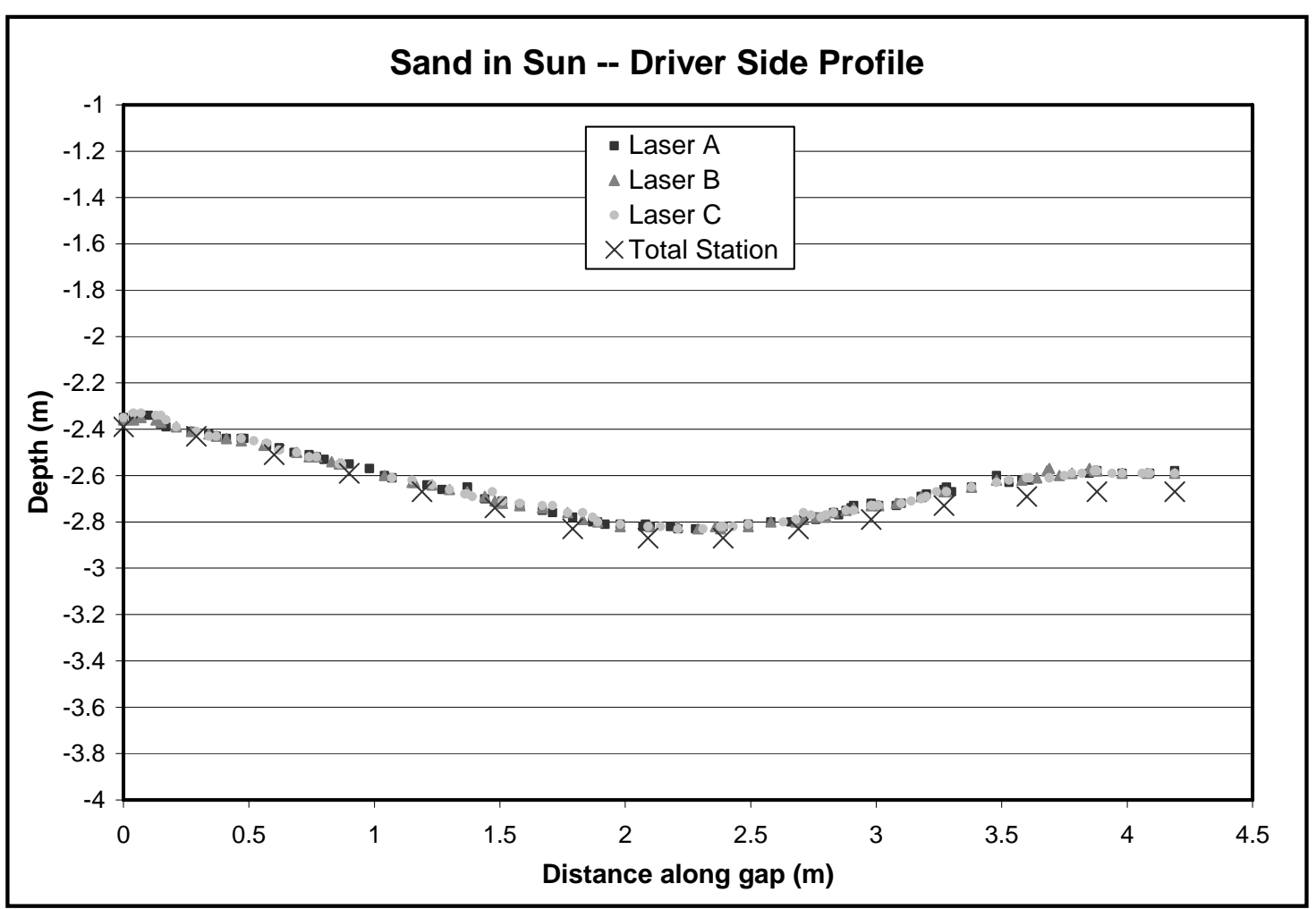

Figure 106. Data for sand gap taken in the sun - driver side profile.

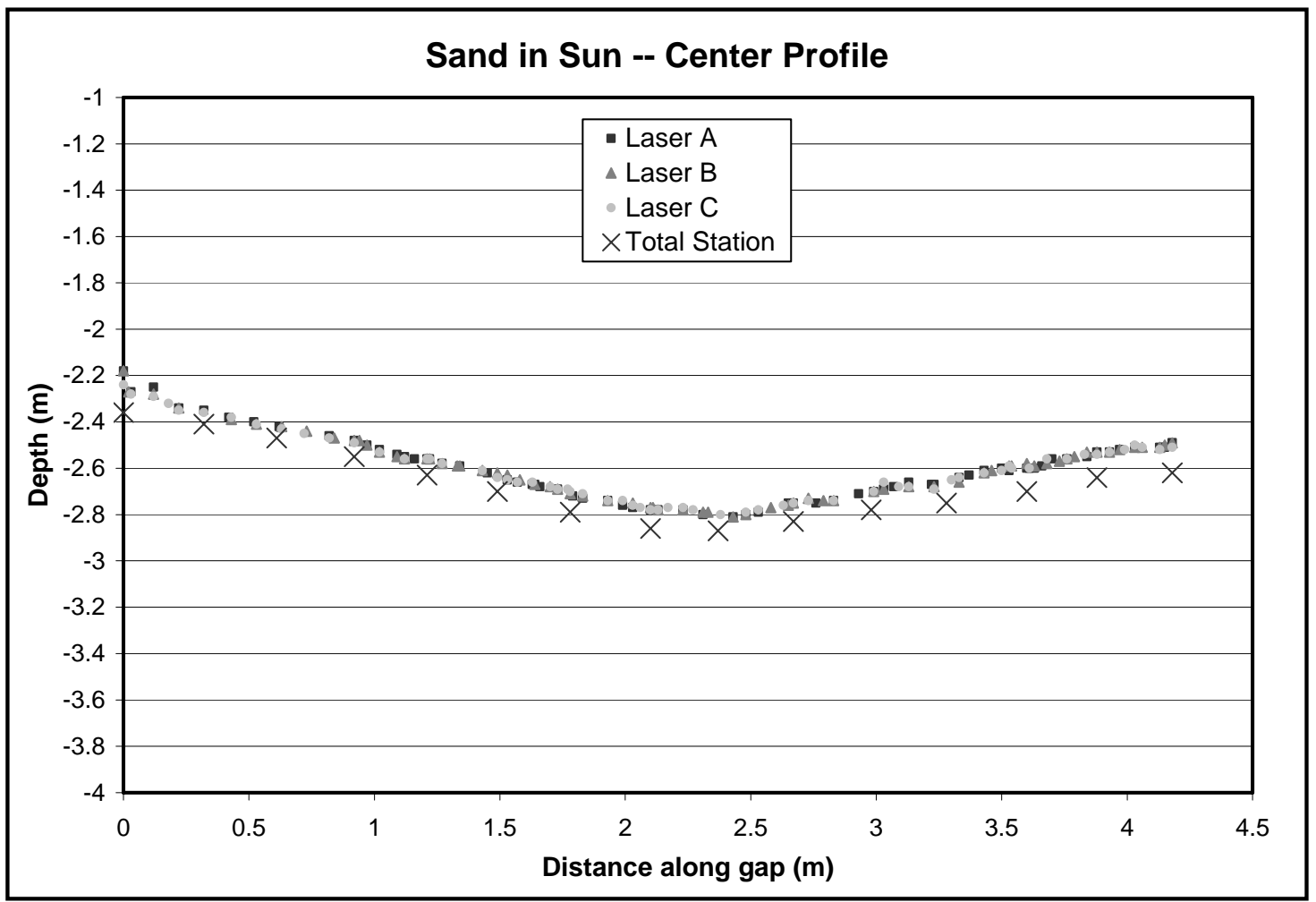

Figure 107. Data for sand gap taken in the sun - center profile. 


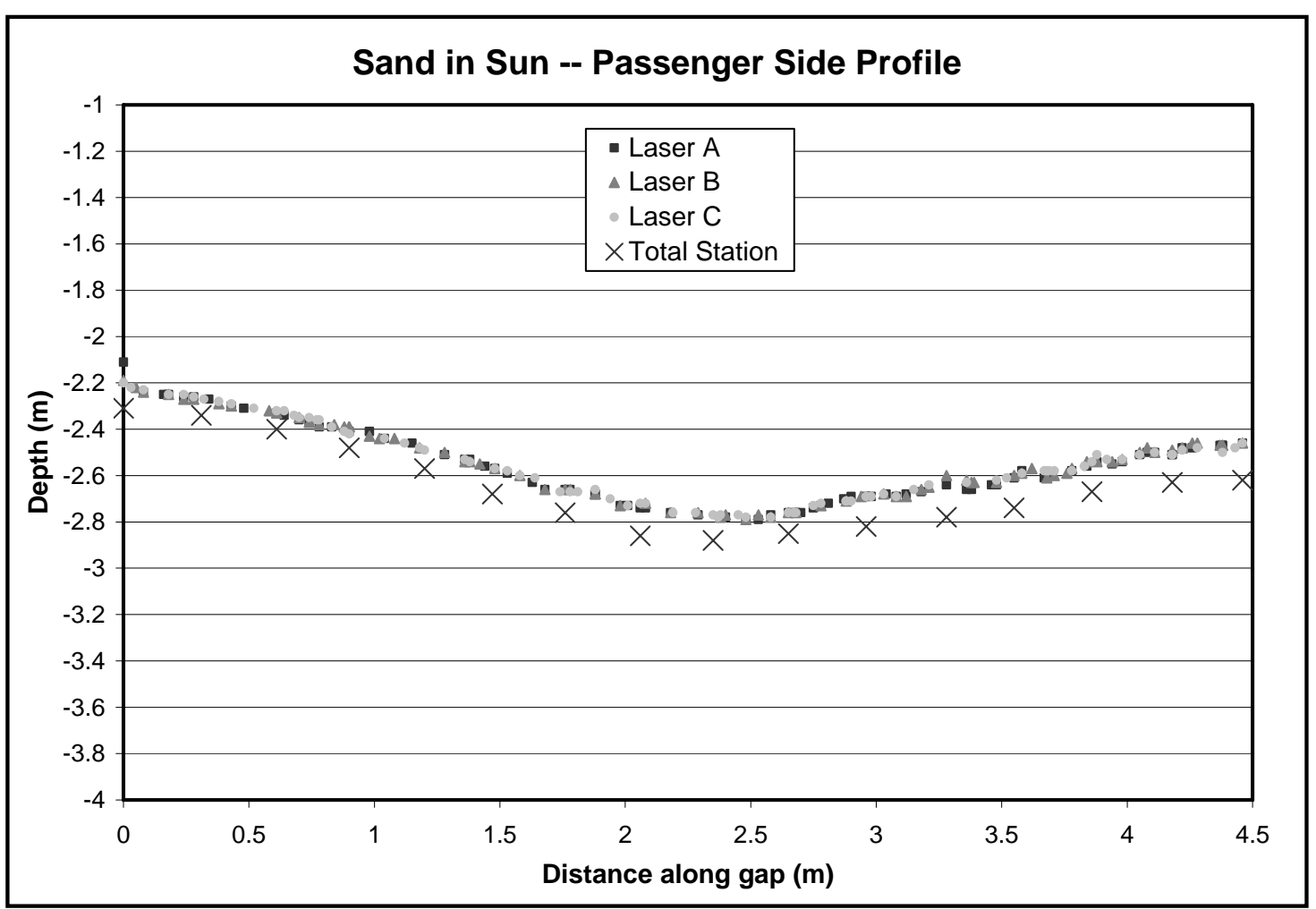

Figure 108. Data for sand gap taken in the sun - passenger side profile.

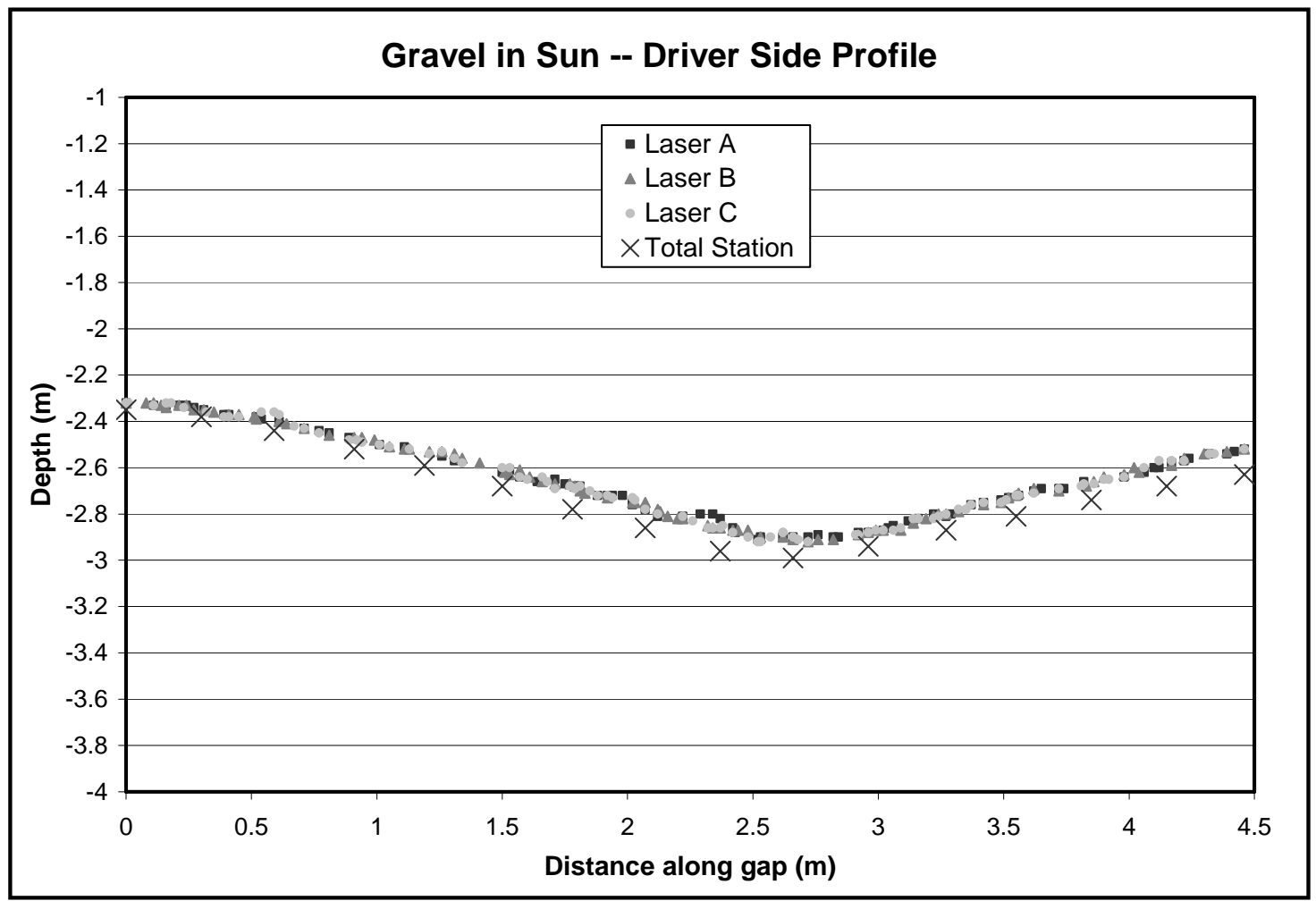

Figure 109. Data for gravel gap taken in the sun - driver side profile. 


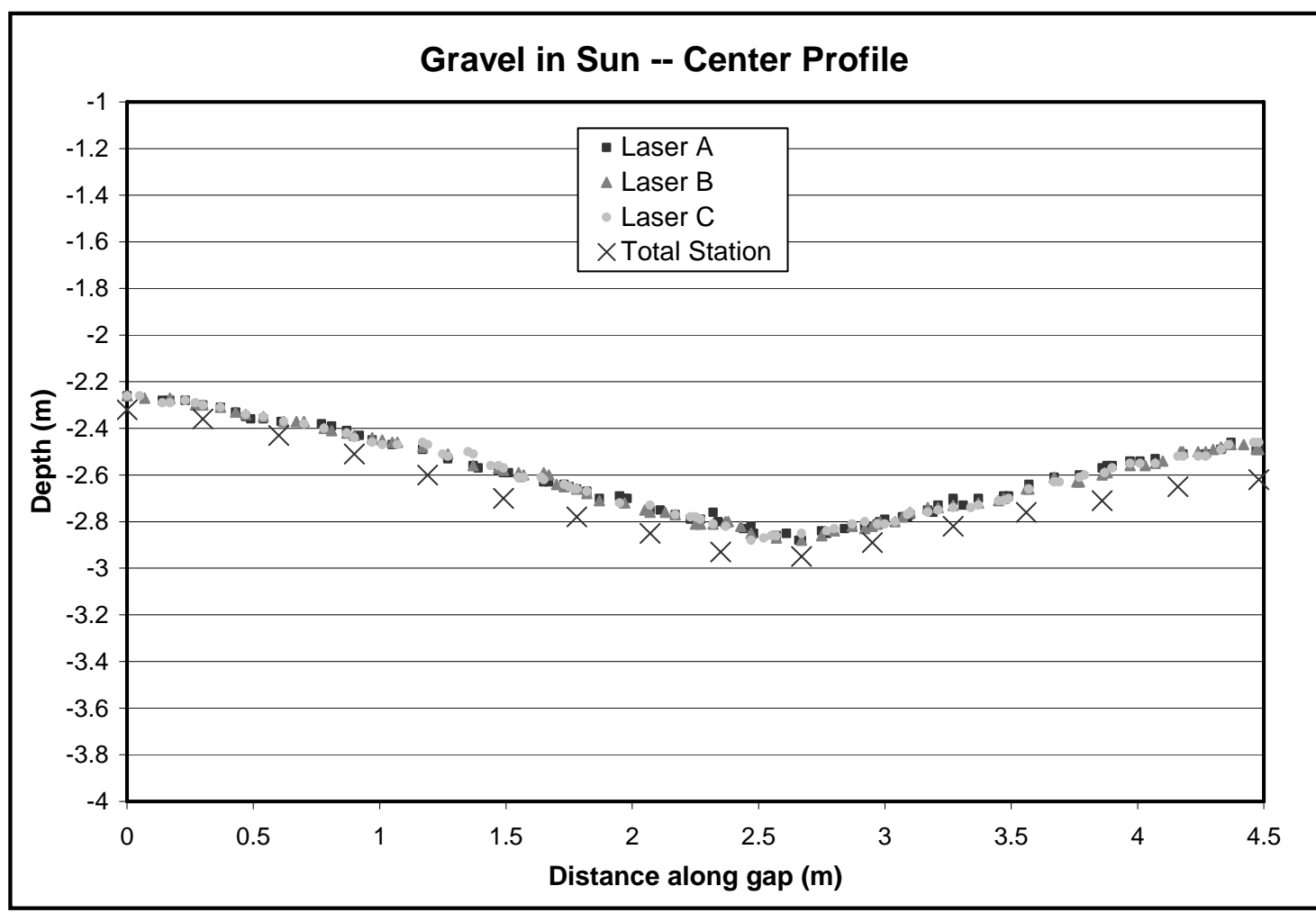

Figure 110. Data for gravel gap taken in the sun - center profile.

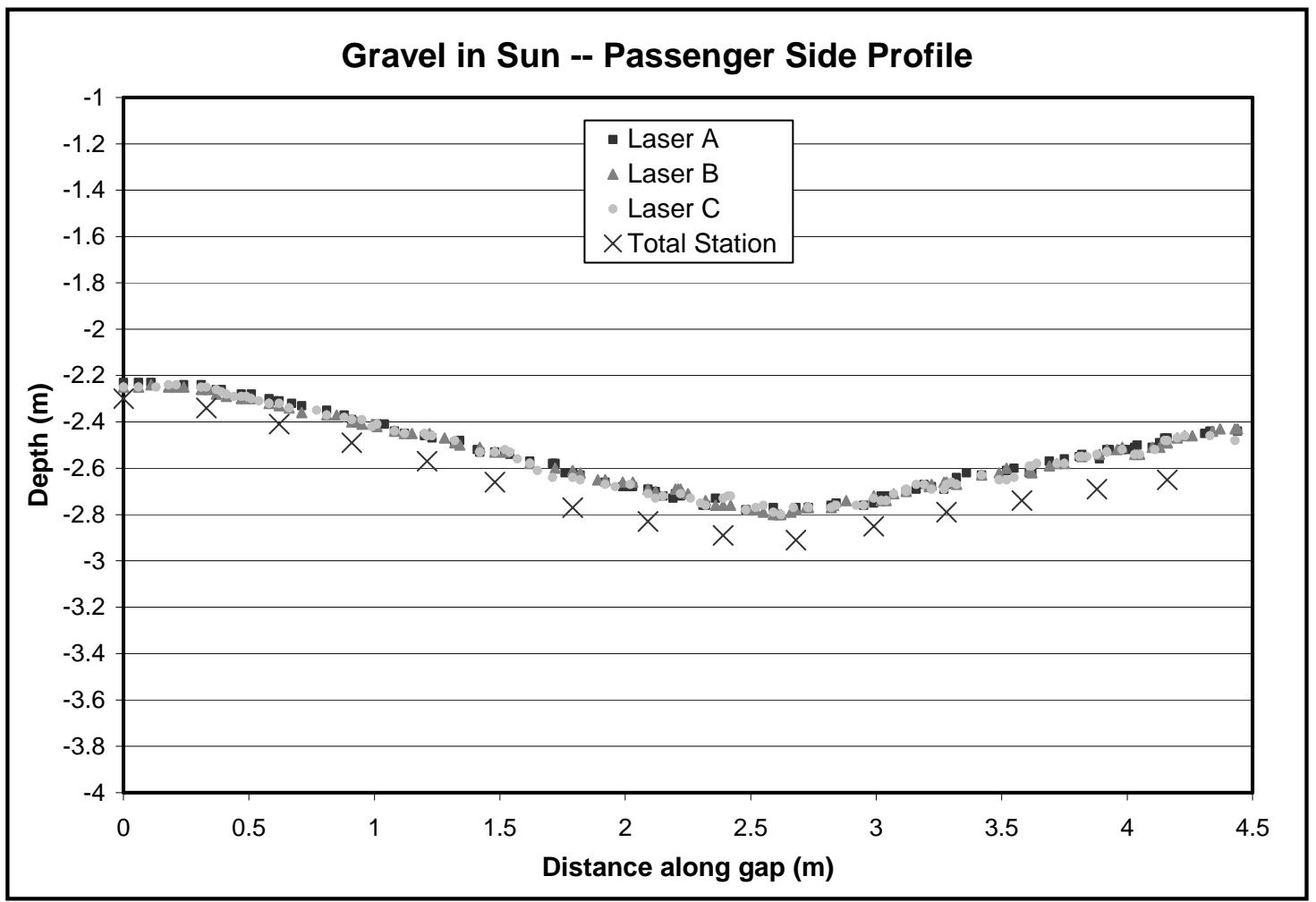

Figure 111. Data for gravel gap taken in the sun - passenger side profile. 


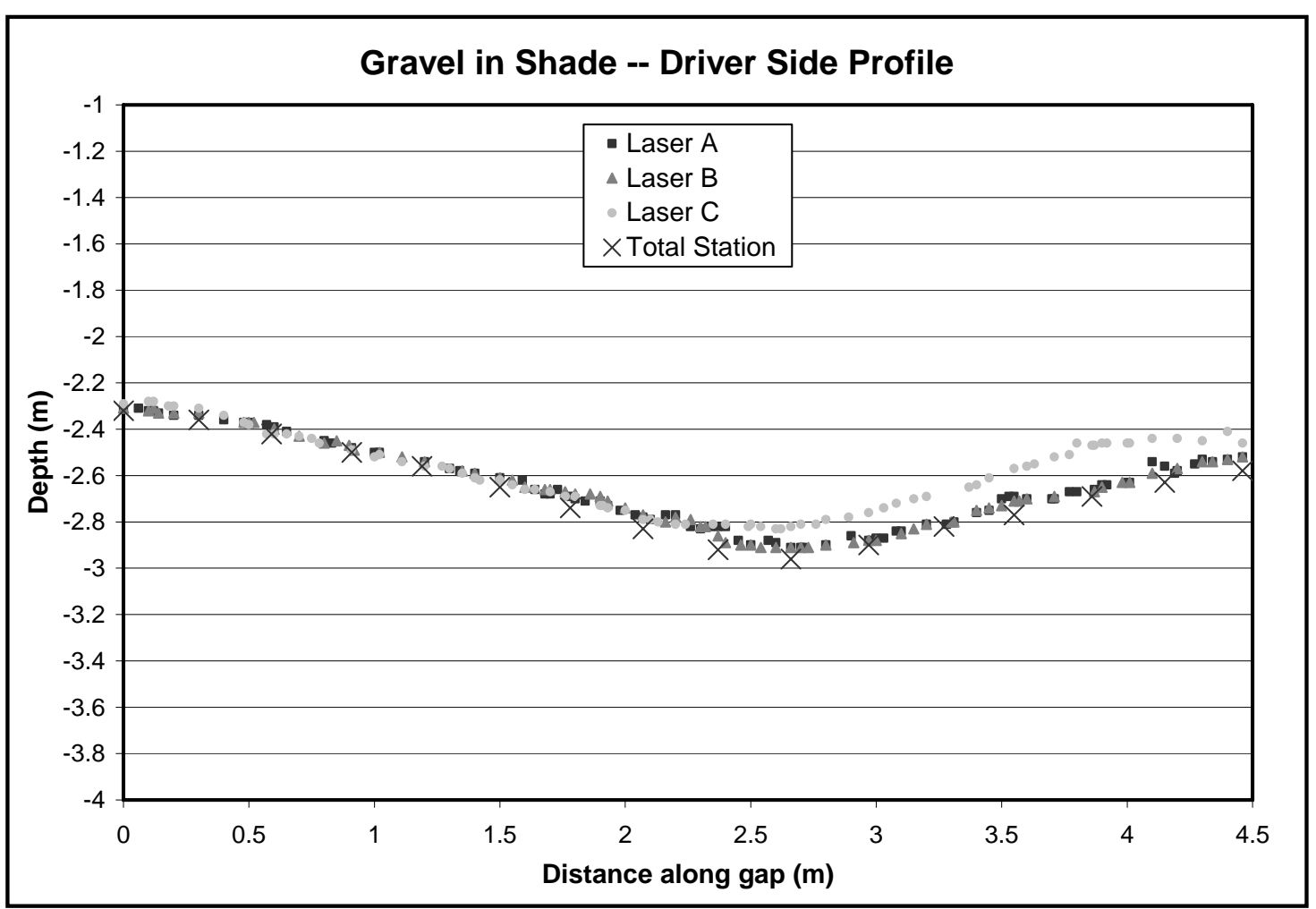

Figure 112. Data for gravel gap taken in the shade - driver side profile.

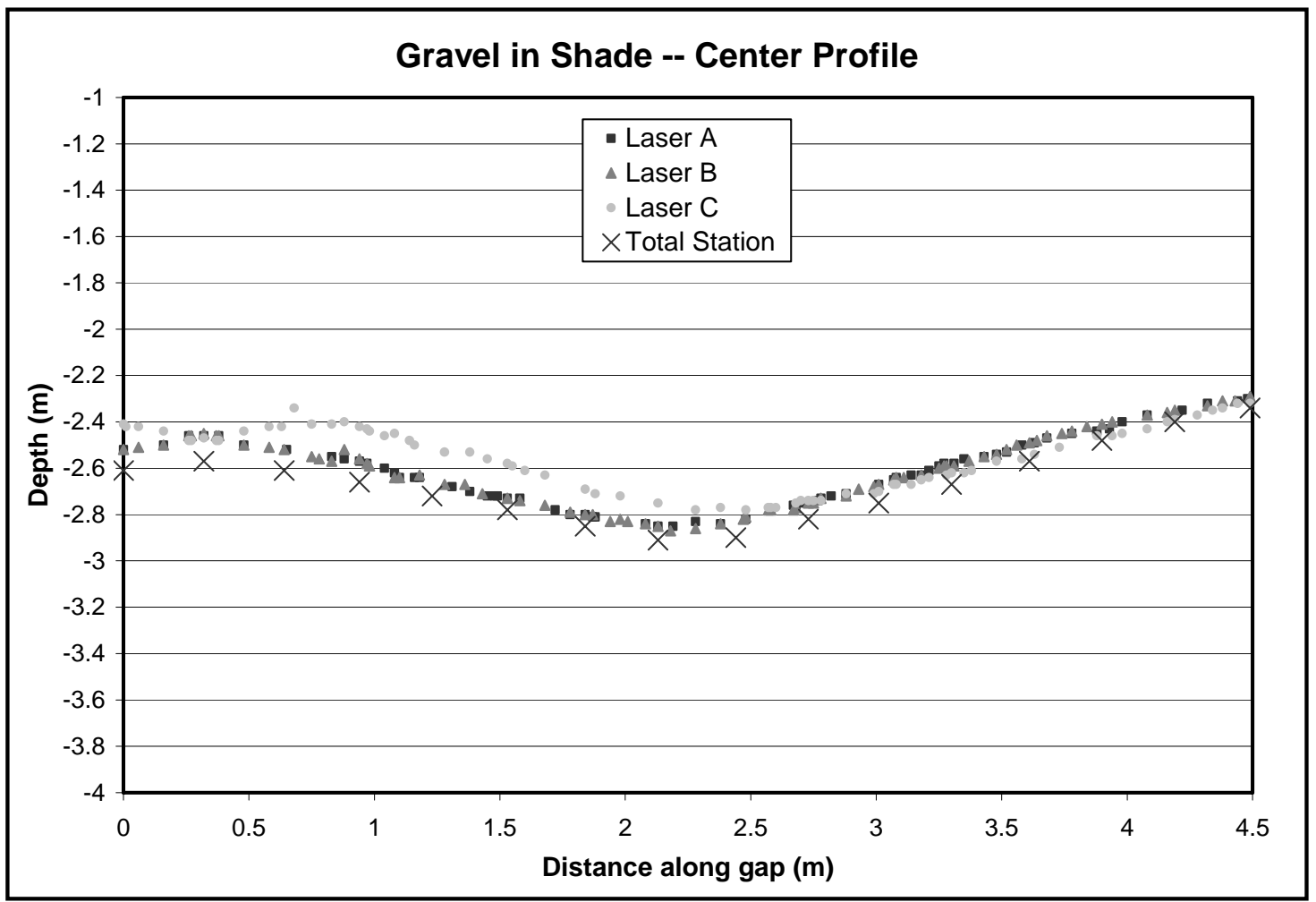

Figure 113. Data for gravel gap taken in the shade - center profile. 


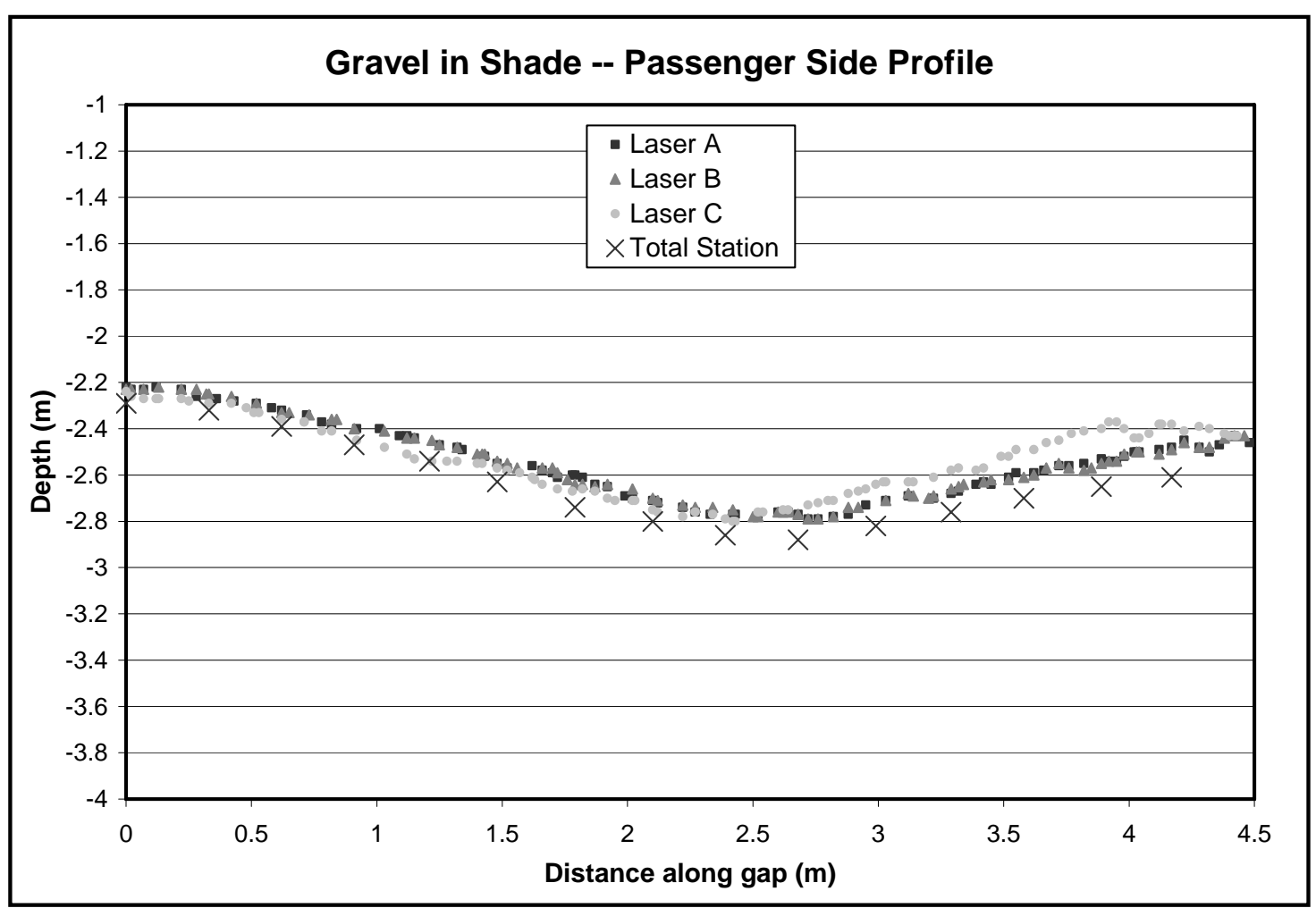

Figure 114. Data for gravel gap taken in the shade - passenger side profile.

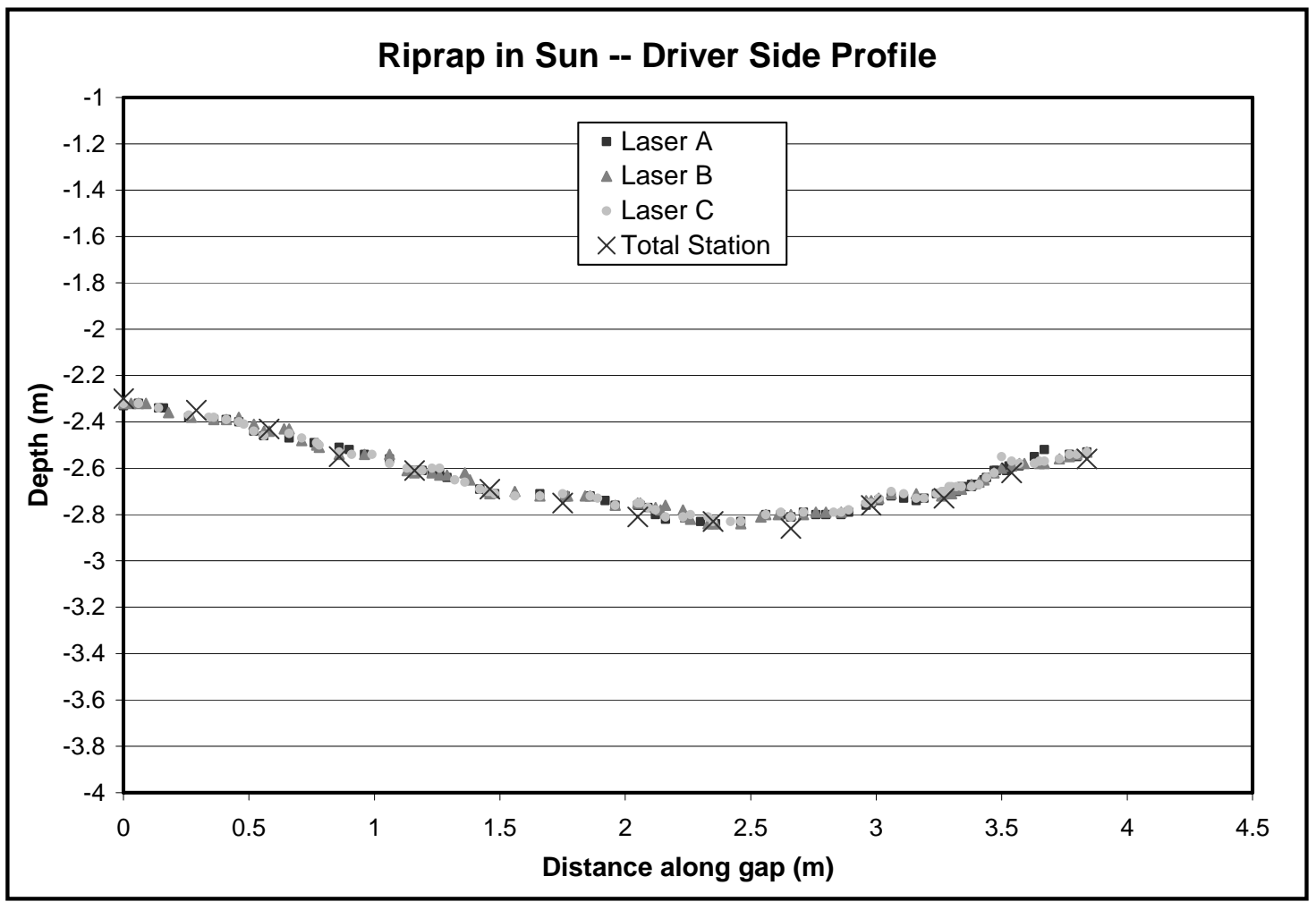

Figure 115. Data for riprap gap taken in the sun - driver side profile. 


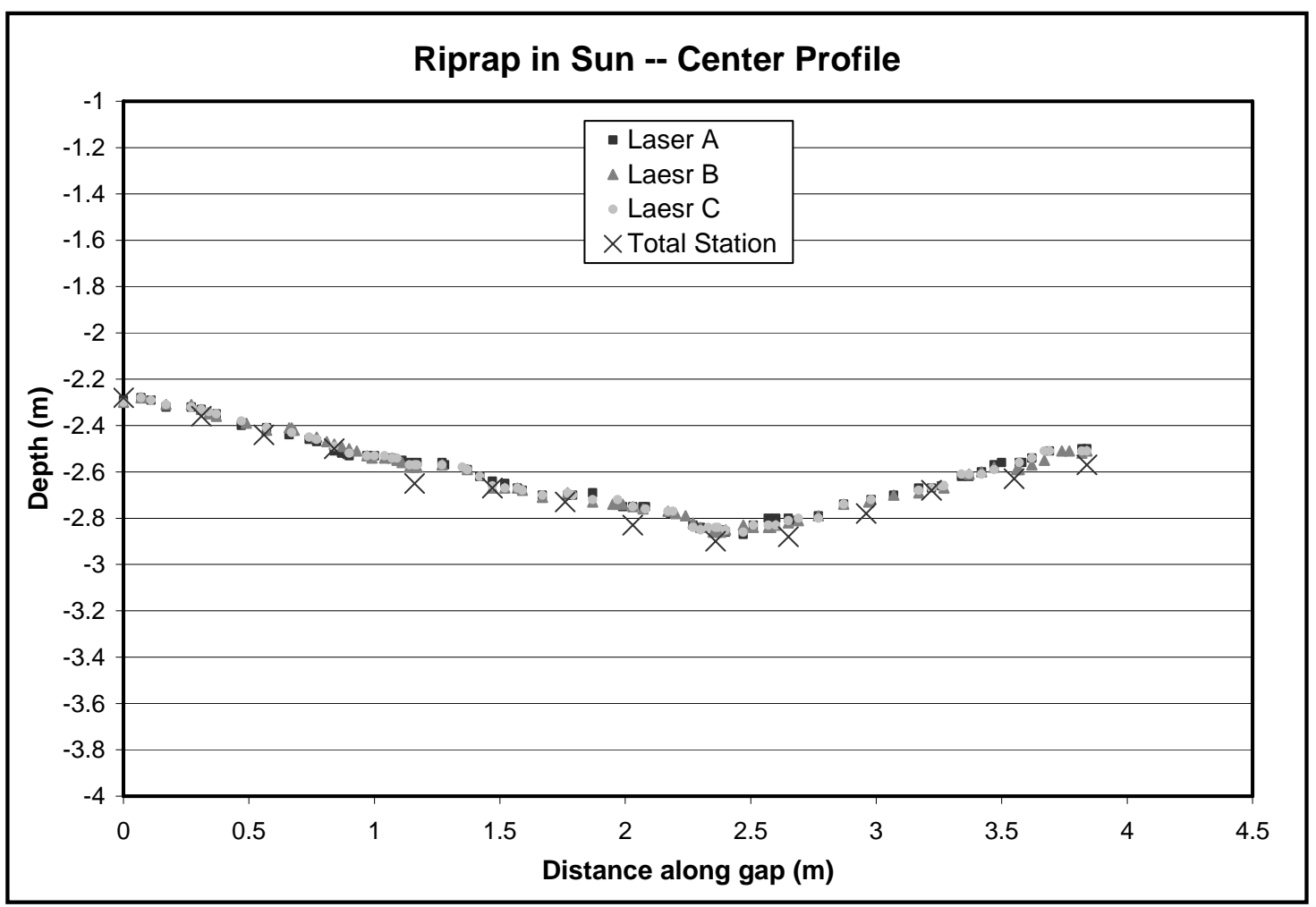

Figure 116. Data for riprap gap taken in the sun - center profile.

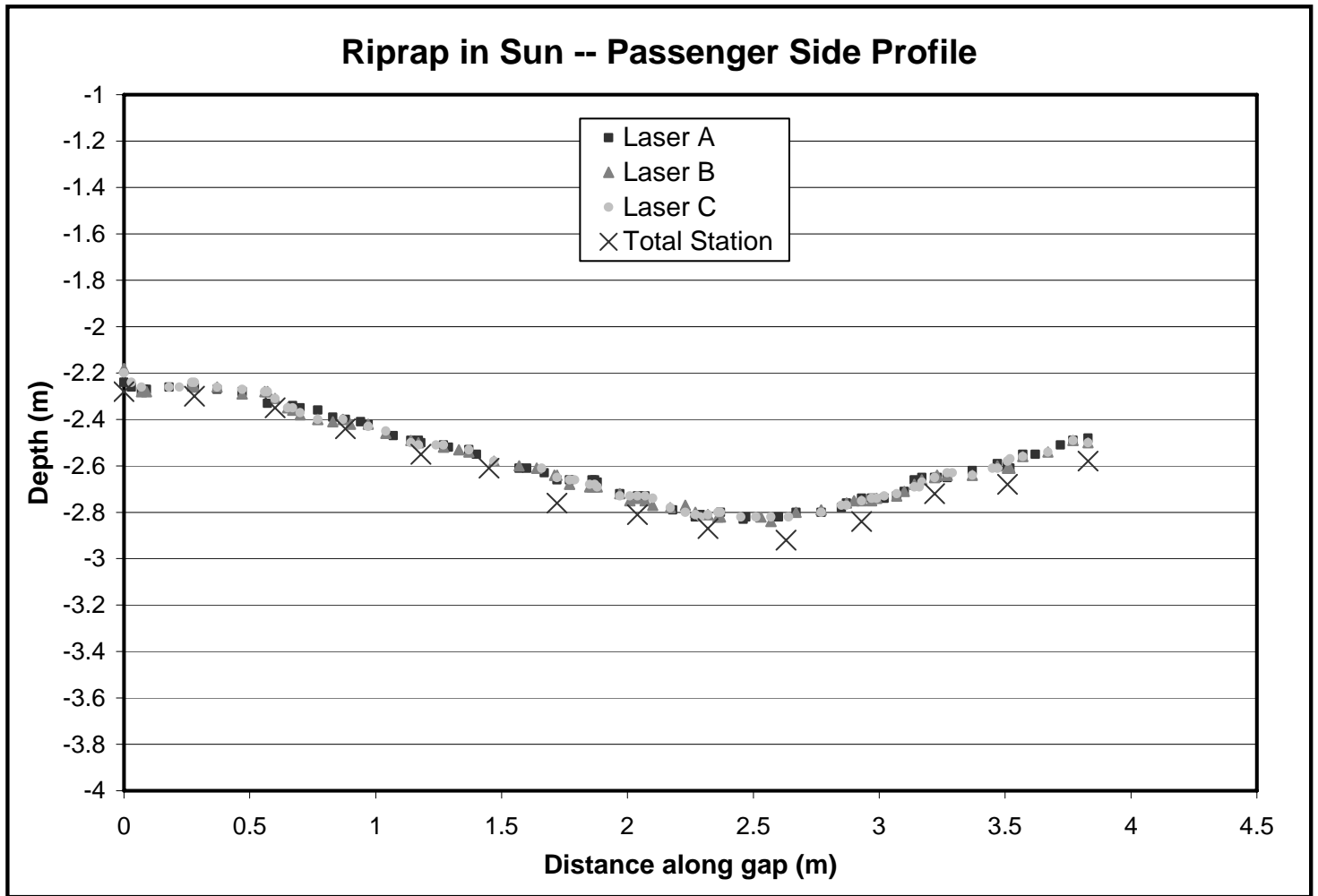

Figure 117. Data for riprap gap taken in the sun - passenger side profile. 


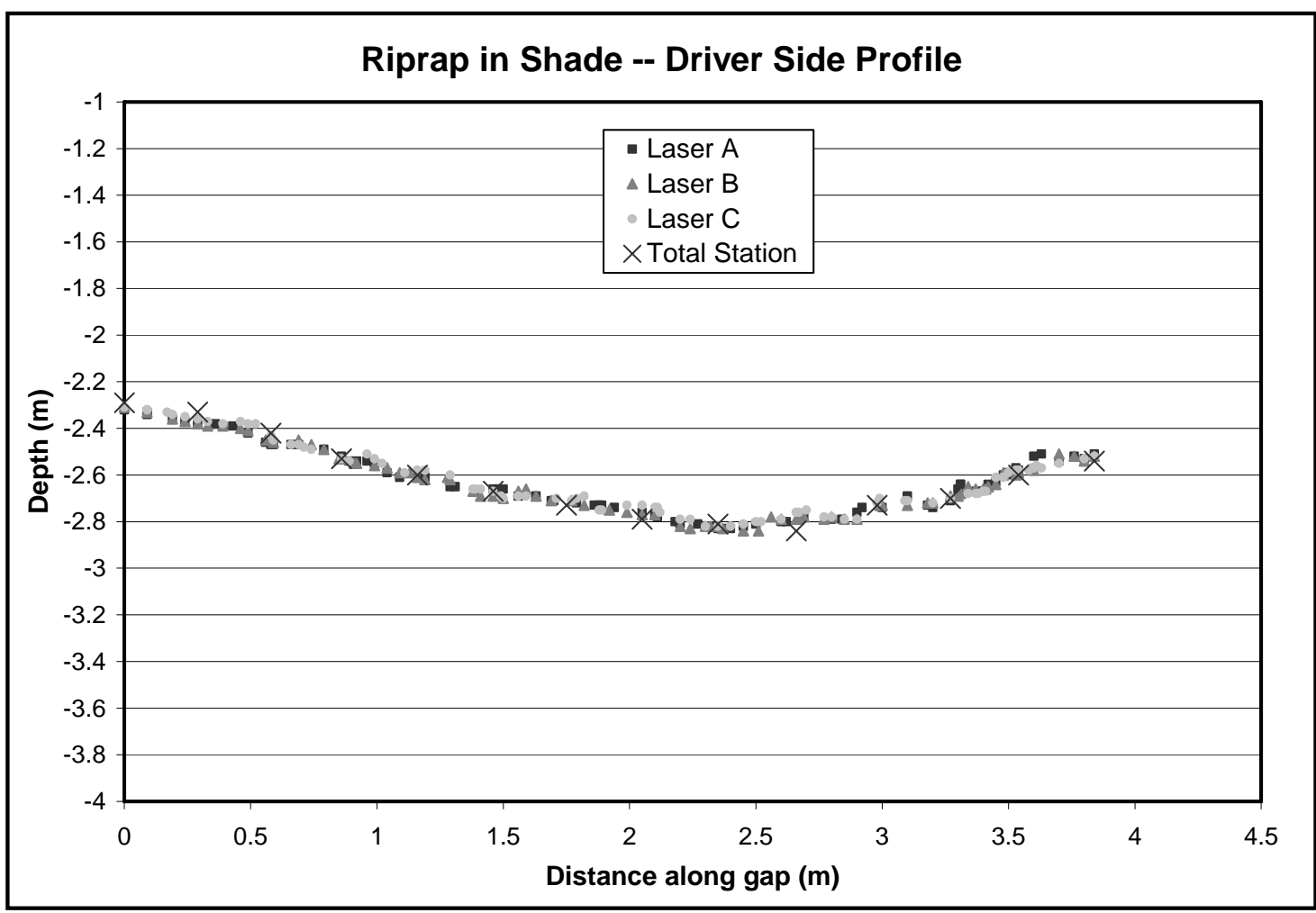

Figure 118. Data for riprap gap taken in the shade - driver side profile.

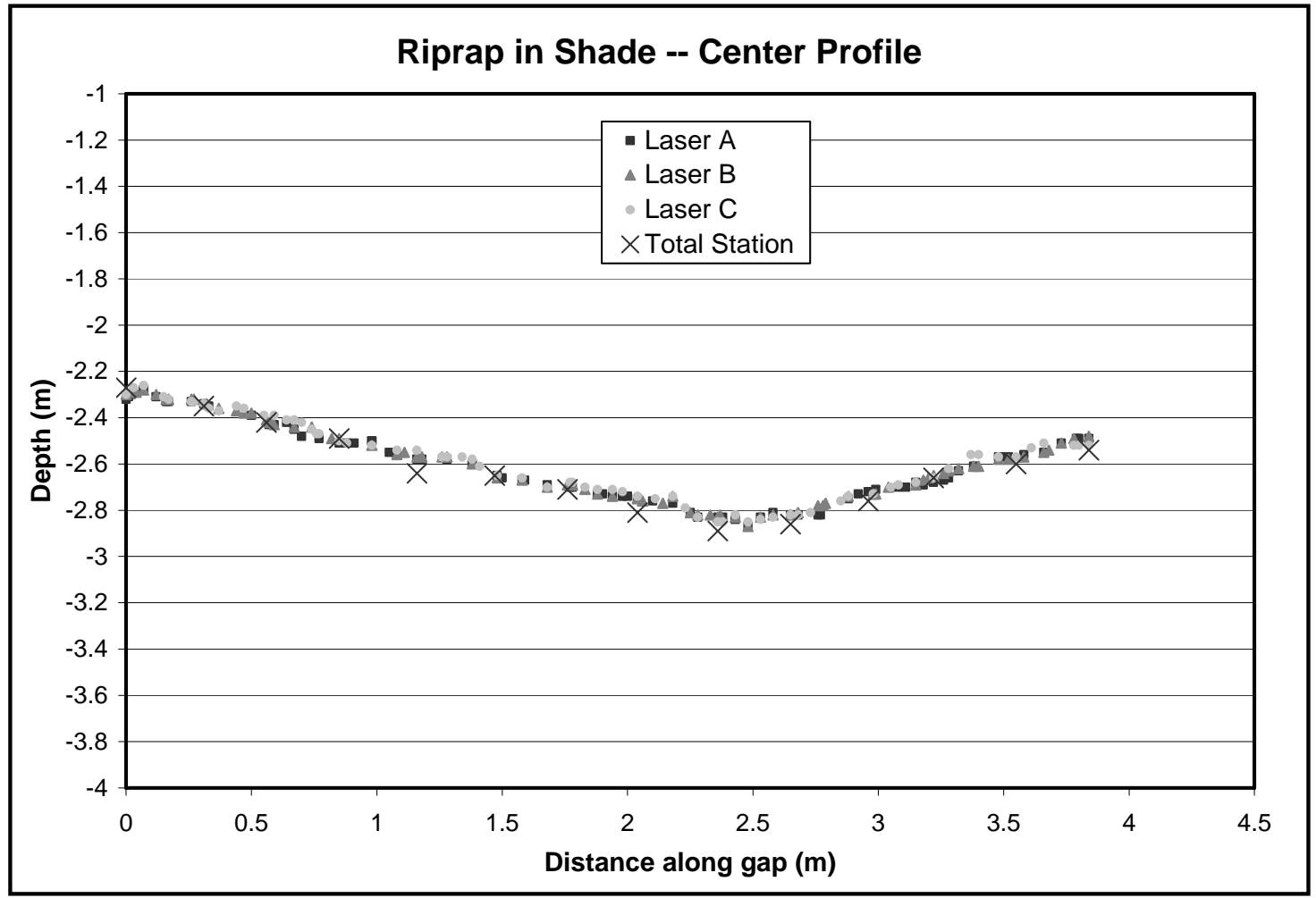

Figure 119. Data for riprap gap taken in the shade - center profile. 


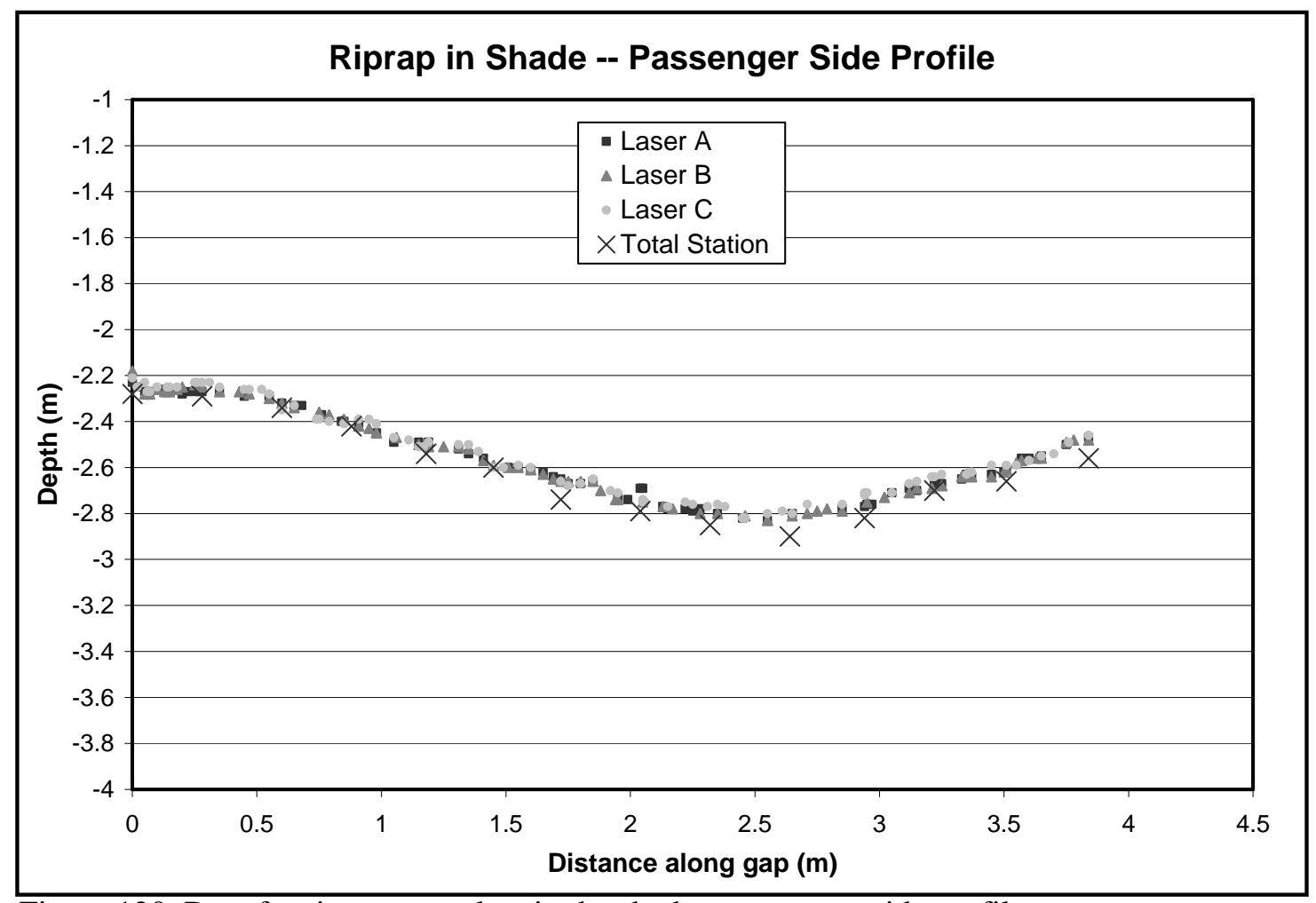

Figure 120. Data for riprap gap taken in the shade - passenger side profile. 


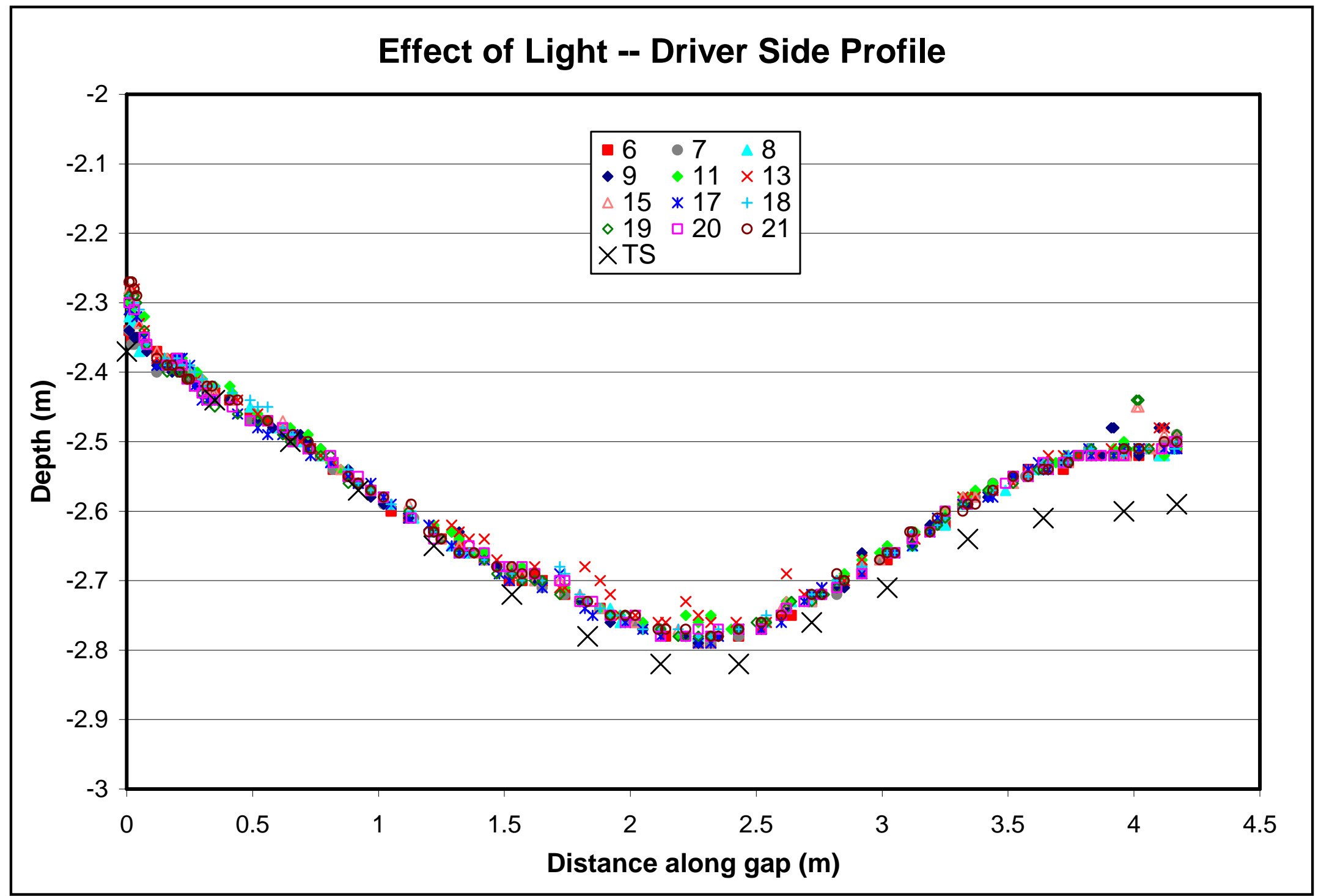

Figure 121. Effect of light on sand gap - driver side profile. 


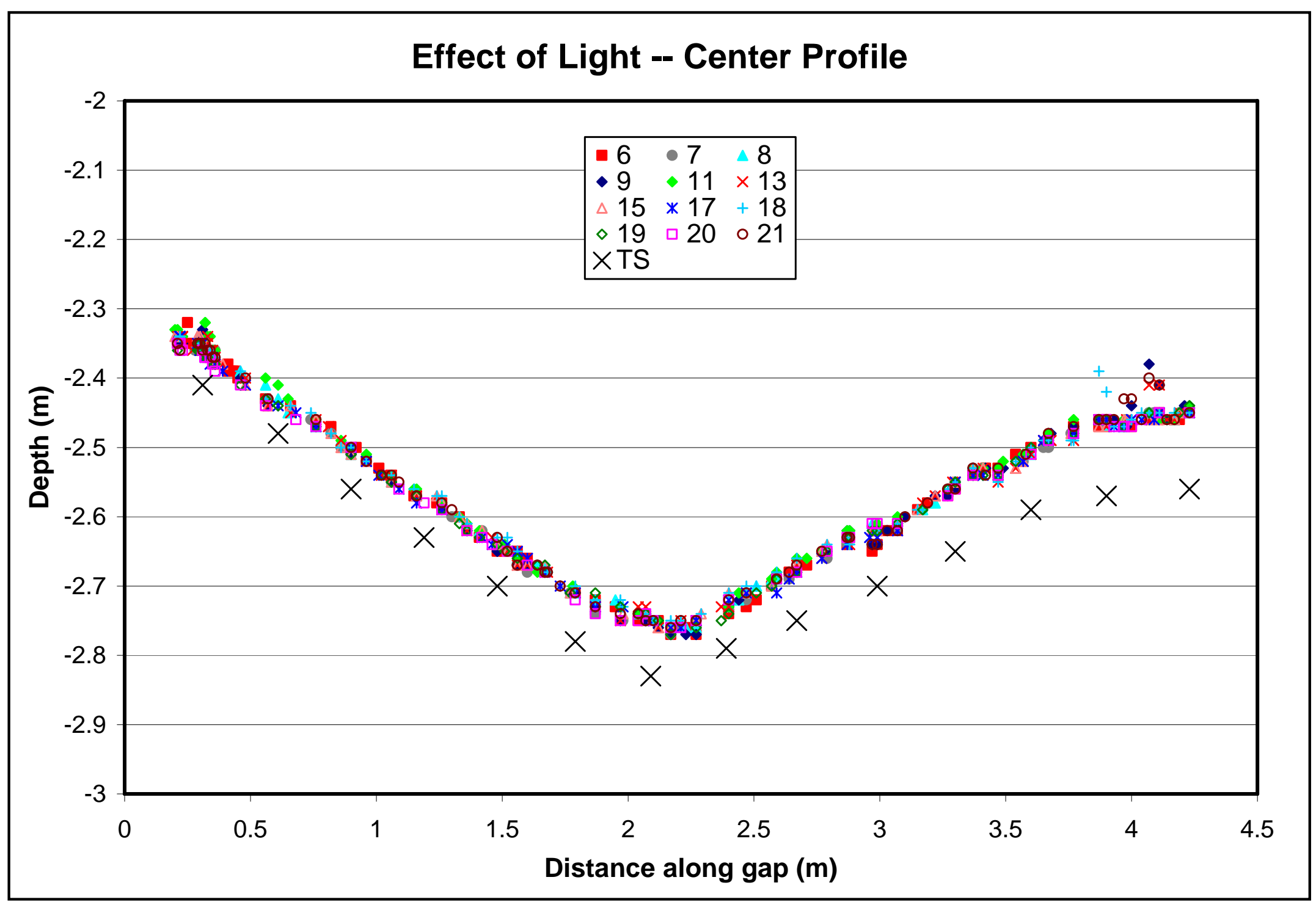

Figure 122. Effect of light on sand gap - center profile. 


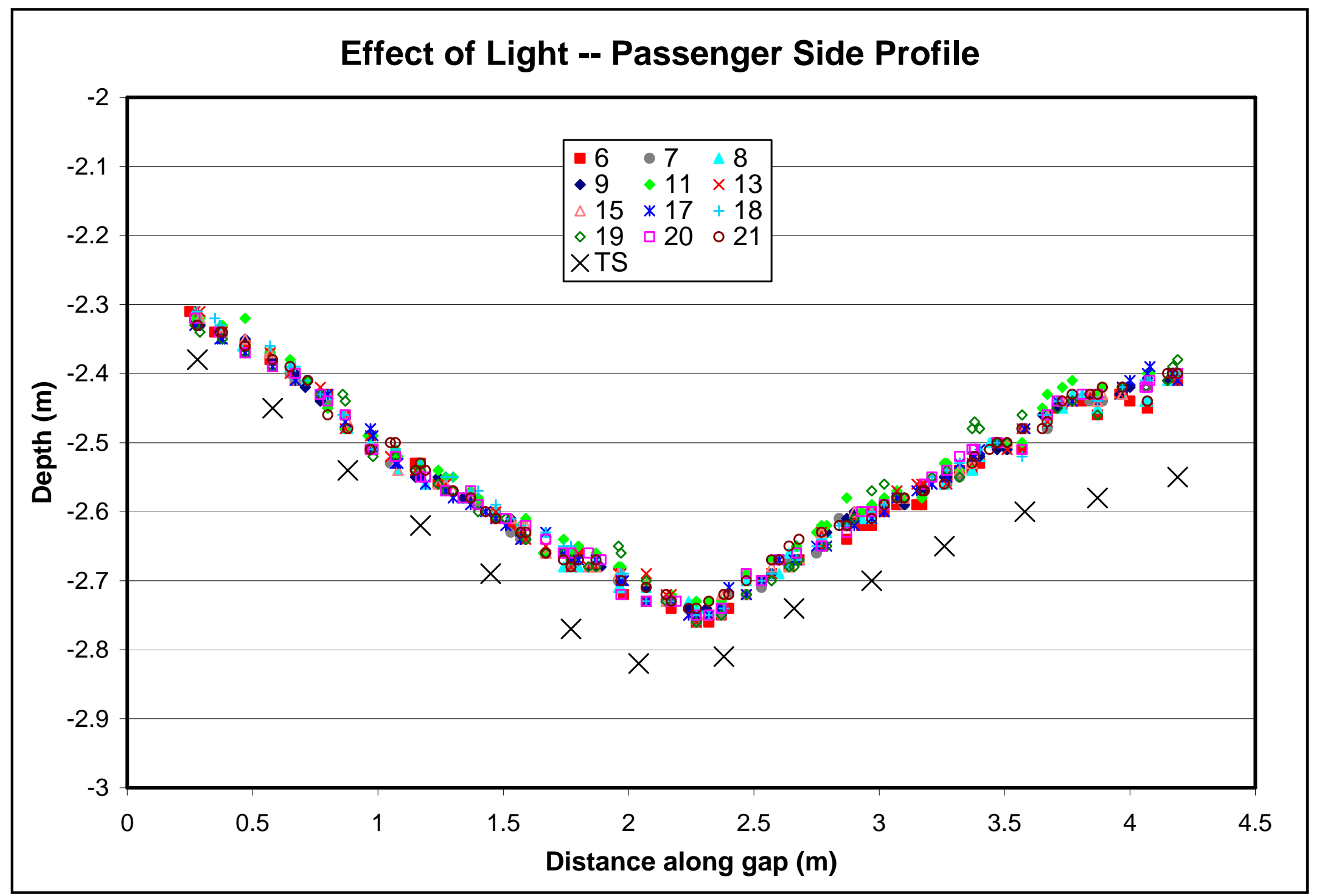

Figure 123. Effect of light on sand gap - passenger side profile. 


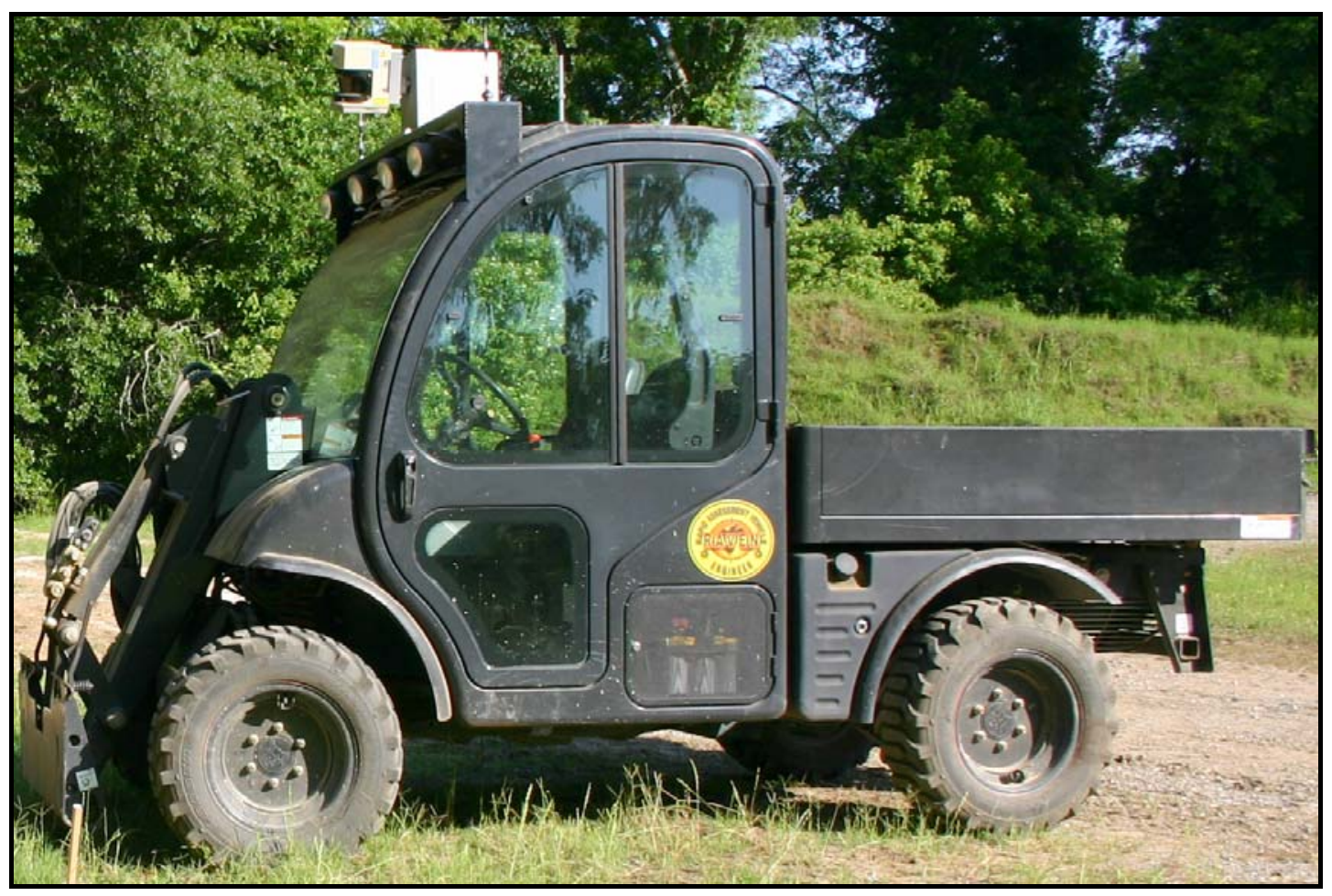

Photo 1. The RAVEN with the 3D LADAR system mounted on top.

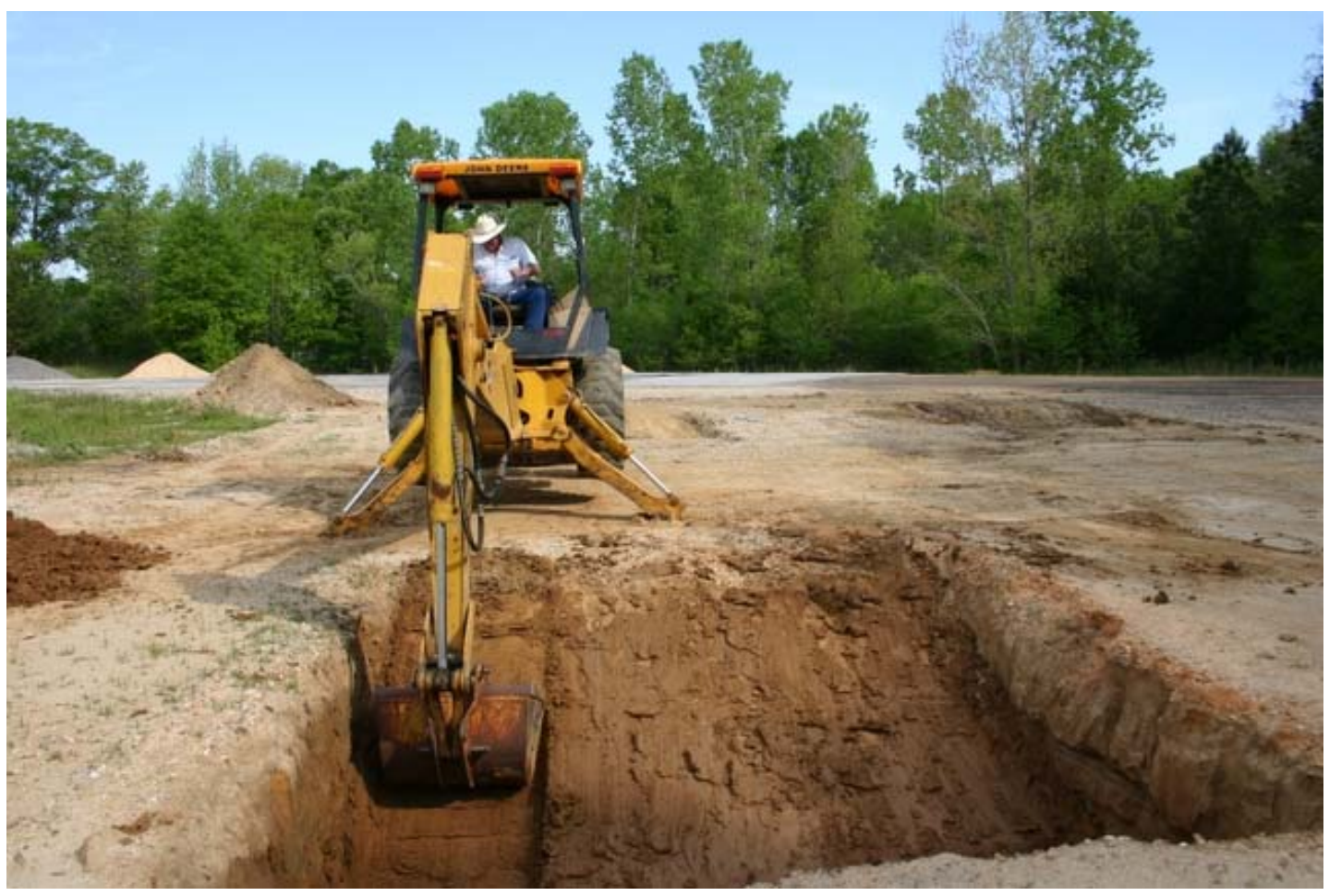

Photo 2. Construction of Gap C. 


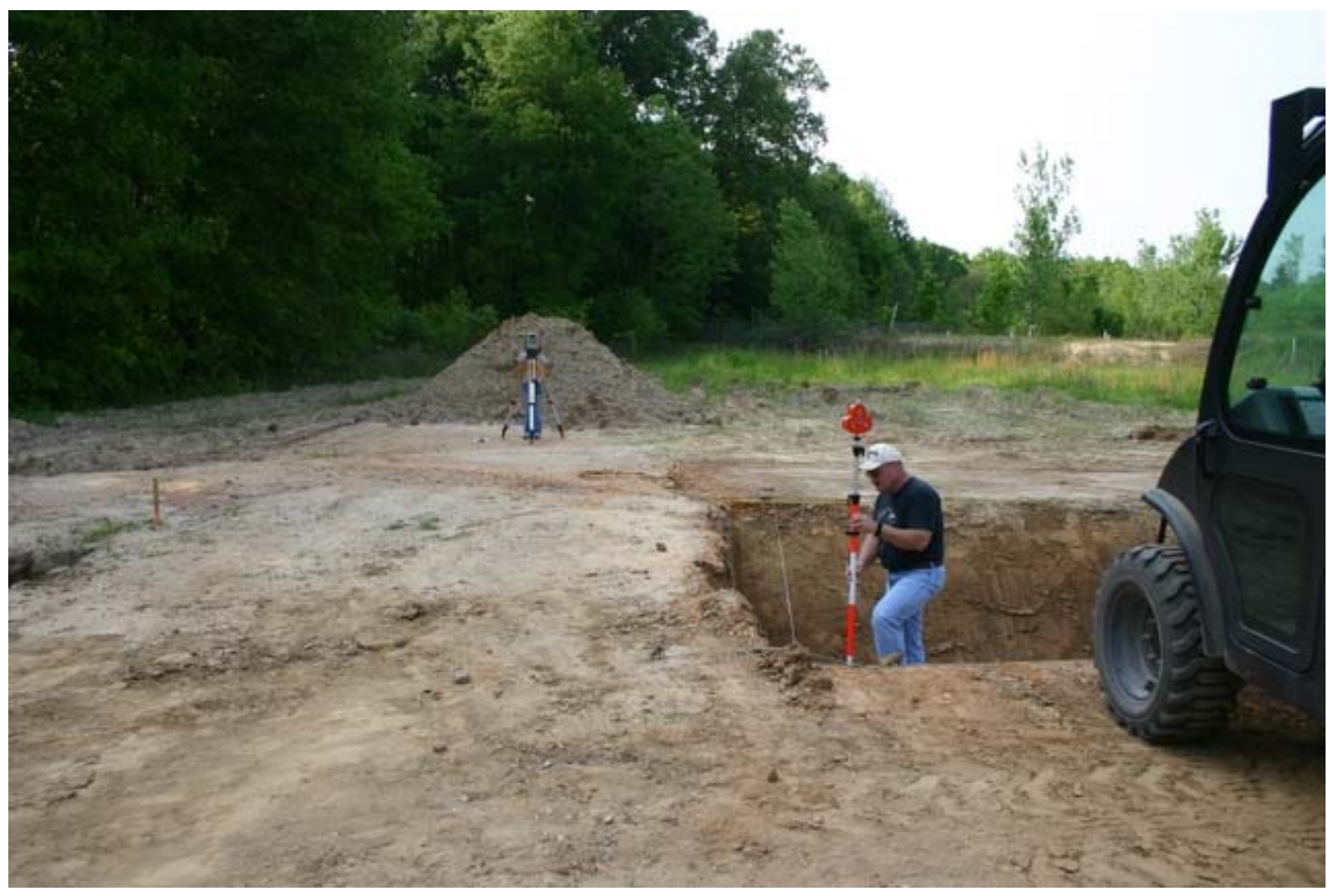

Photo 3. Collecting ground truth total station data.

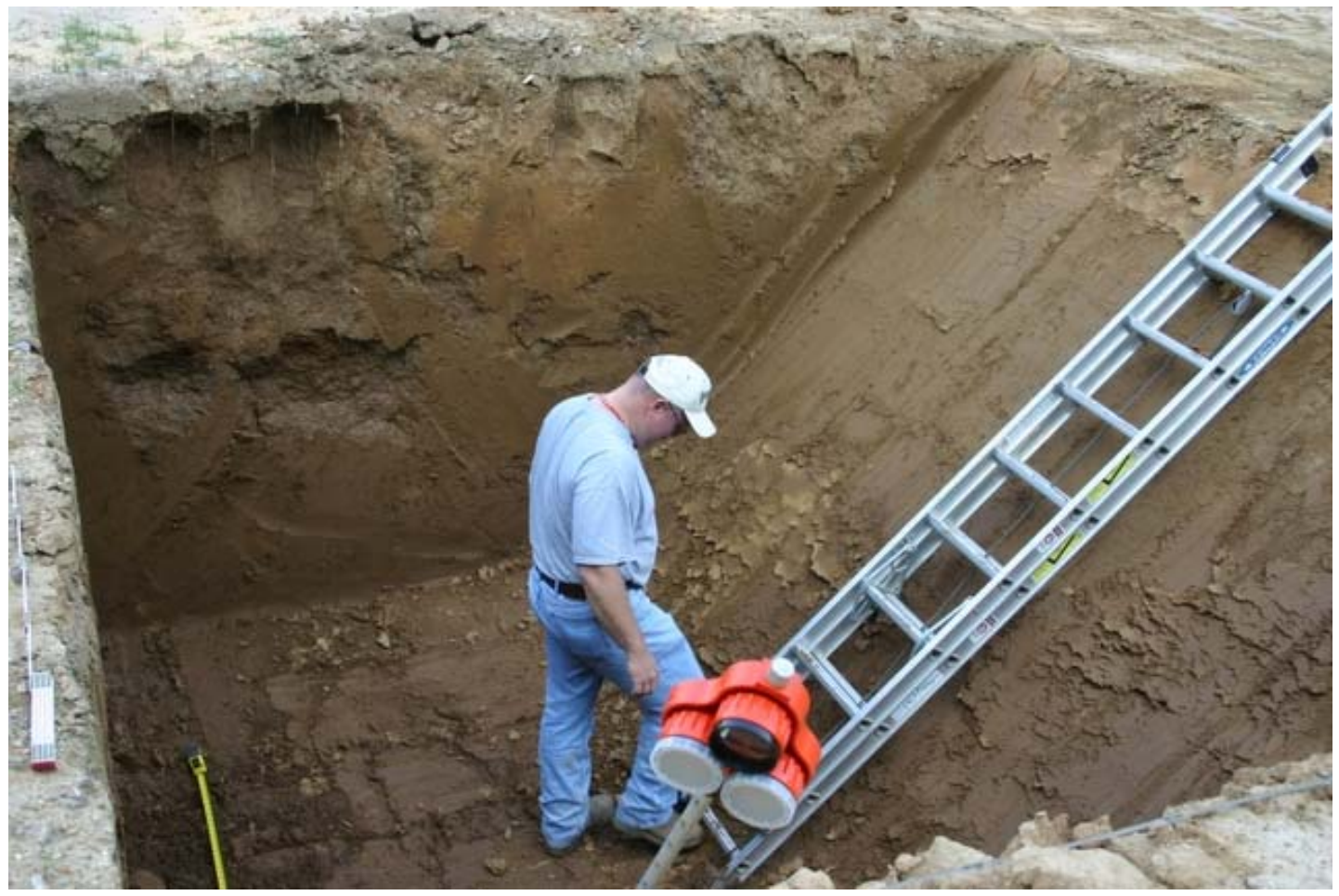

Photo 4. Collecting total station data in Gap D. 


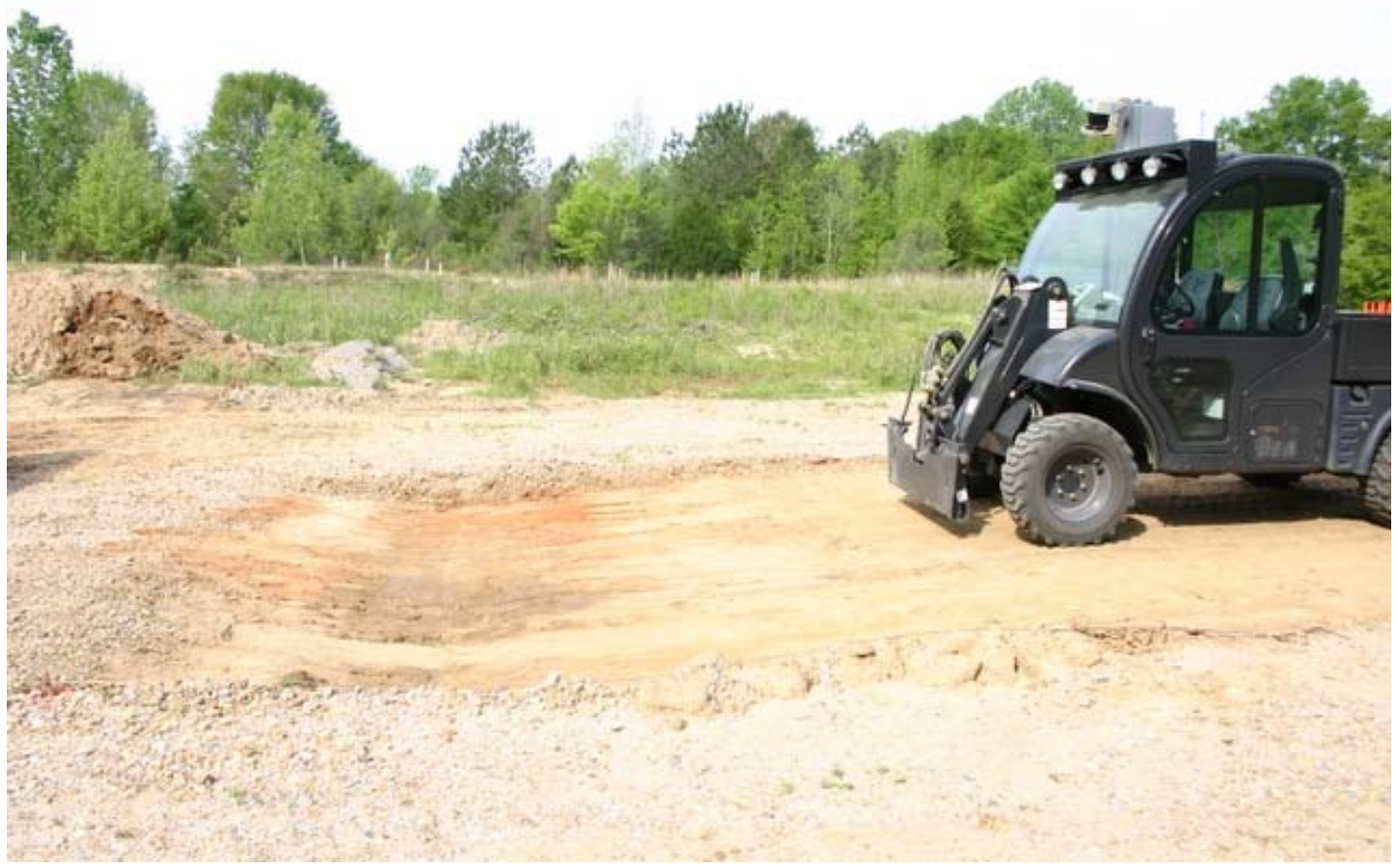

Photo 5. LADAR data collection at Gap A.

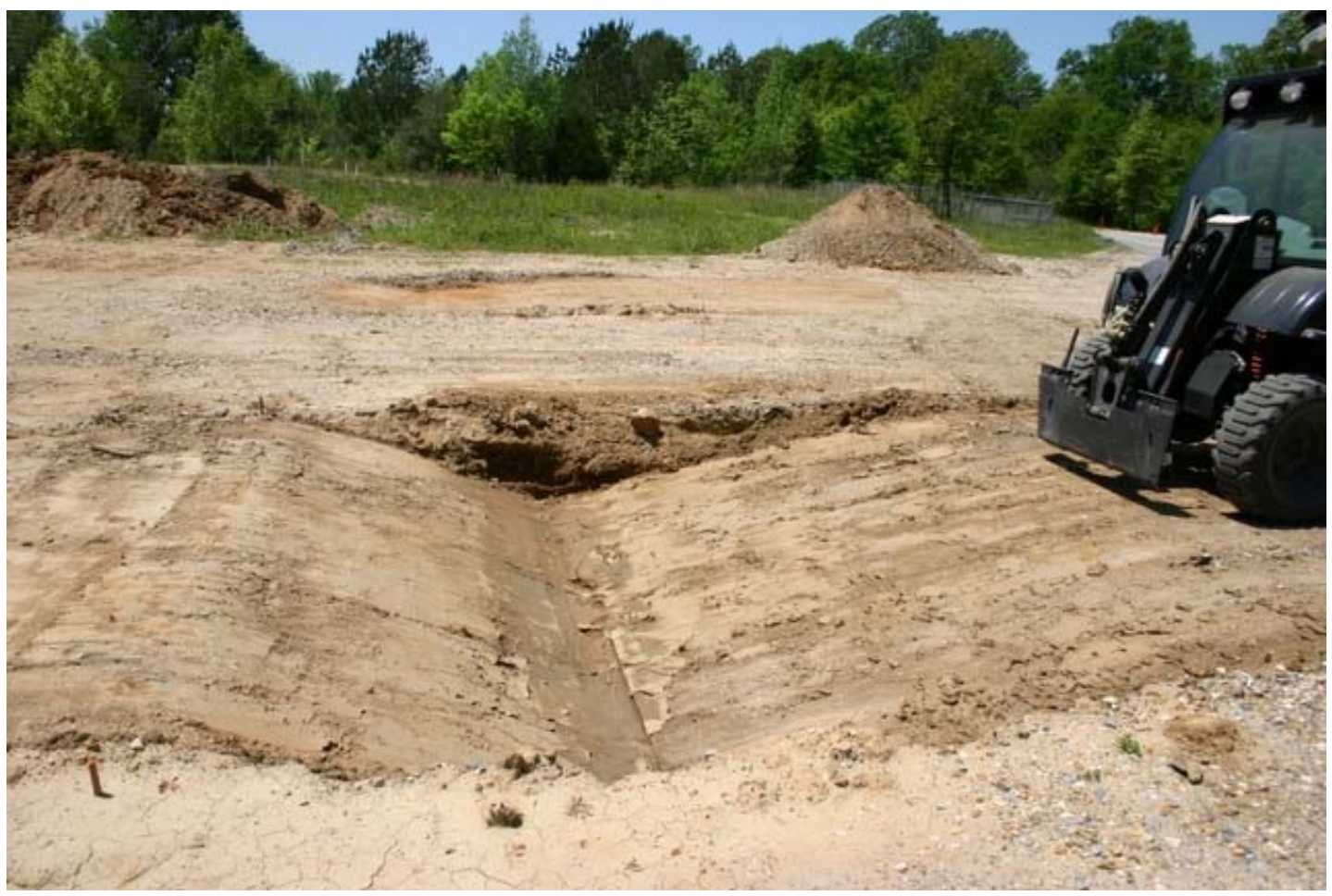

Photo 6. LADAR data collection at Gap B. 


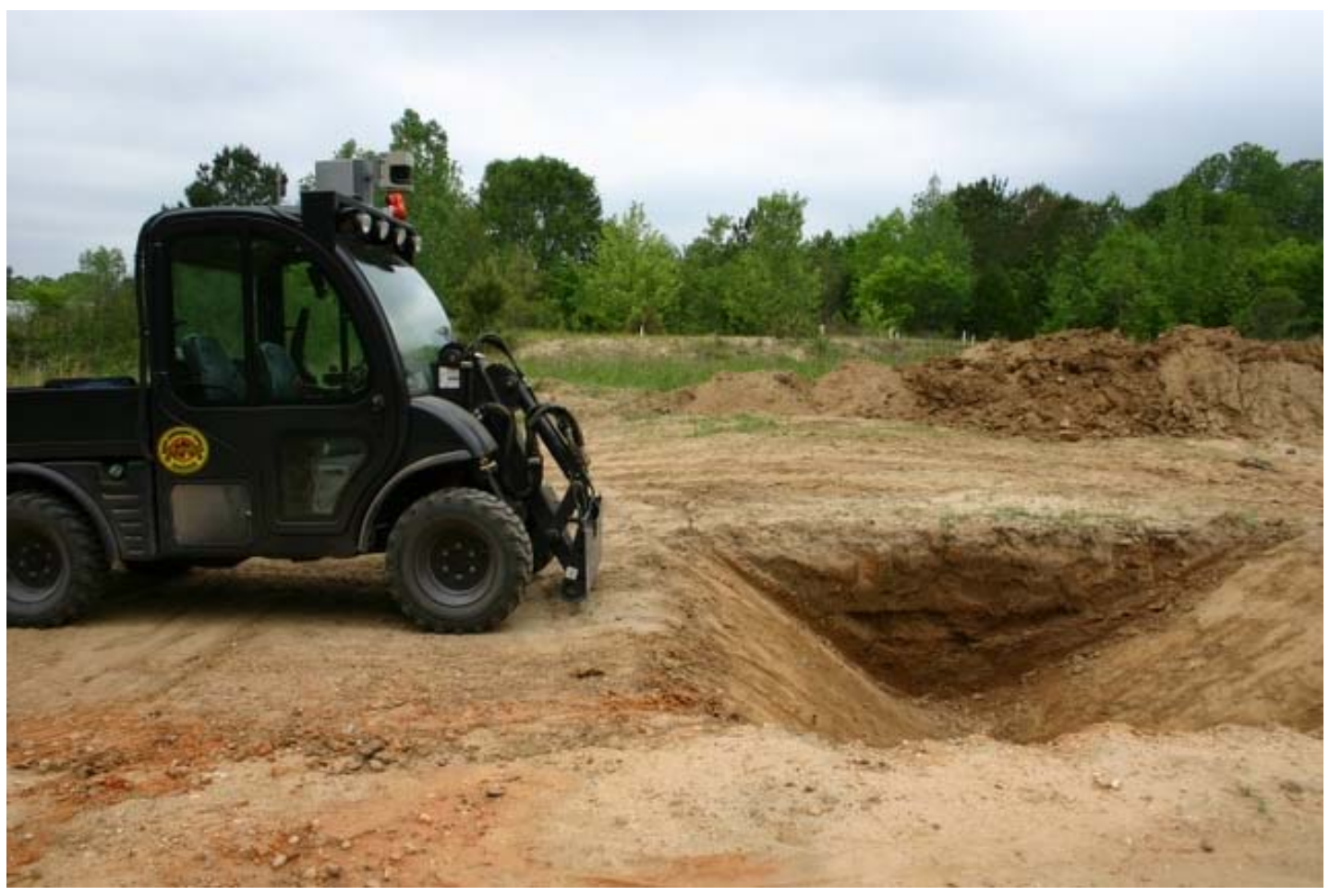

Photo 7. LADAR data collection at Gap C.

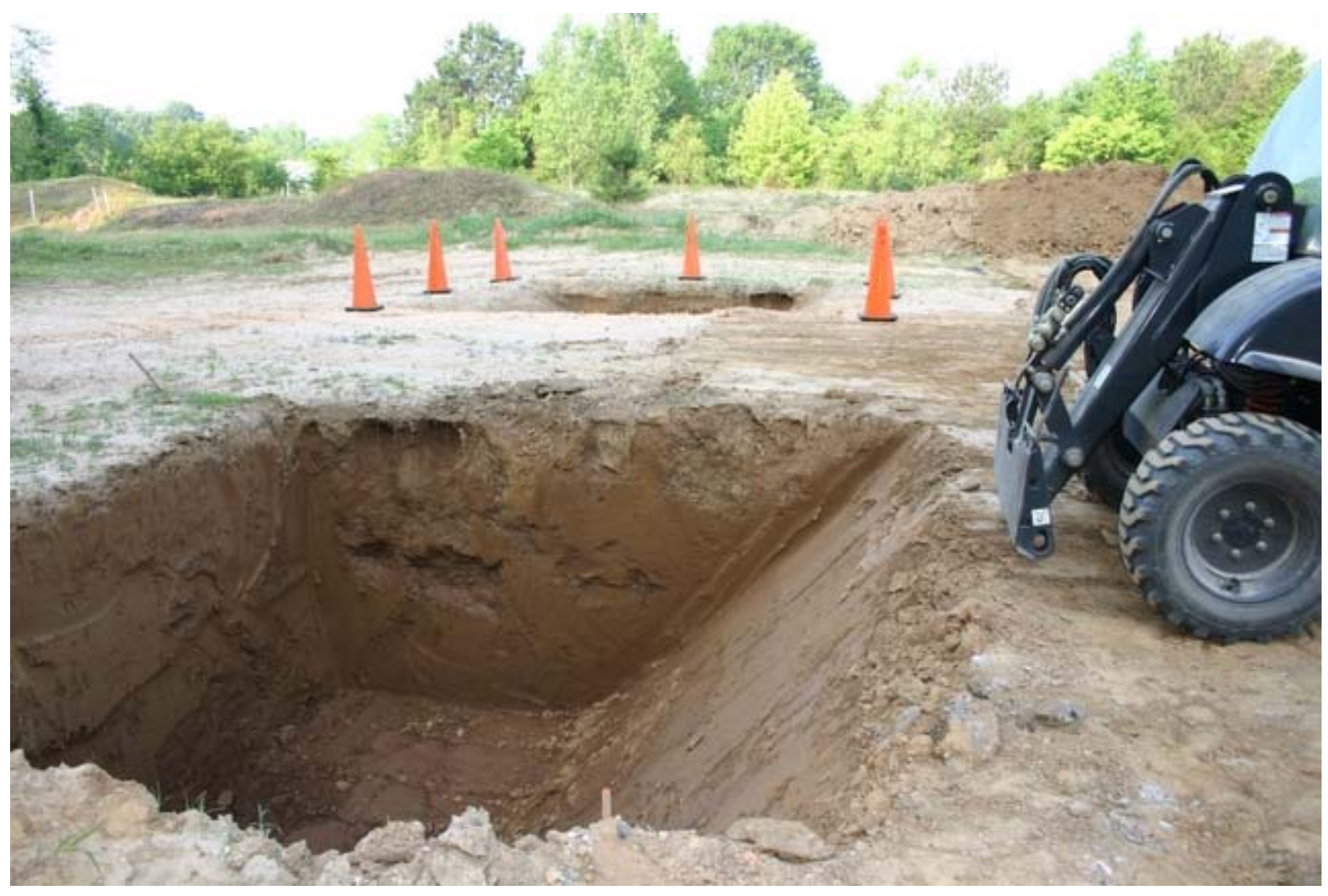

Photo 8. LADAR data collection at Gap D. 


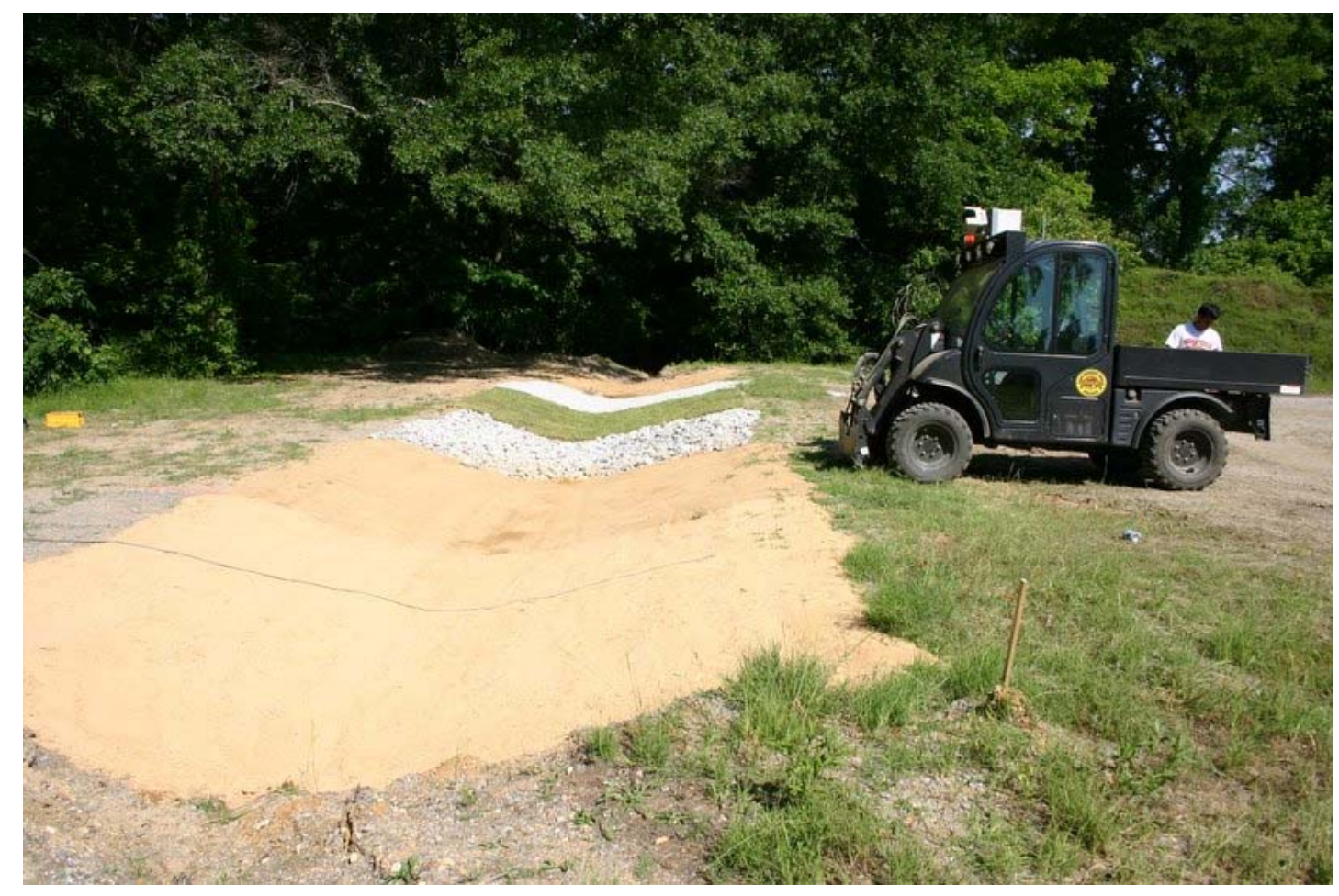

Photo 9. Overview of surface material gap.

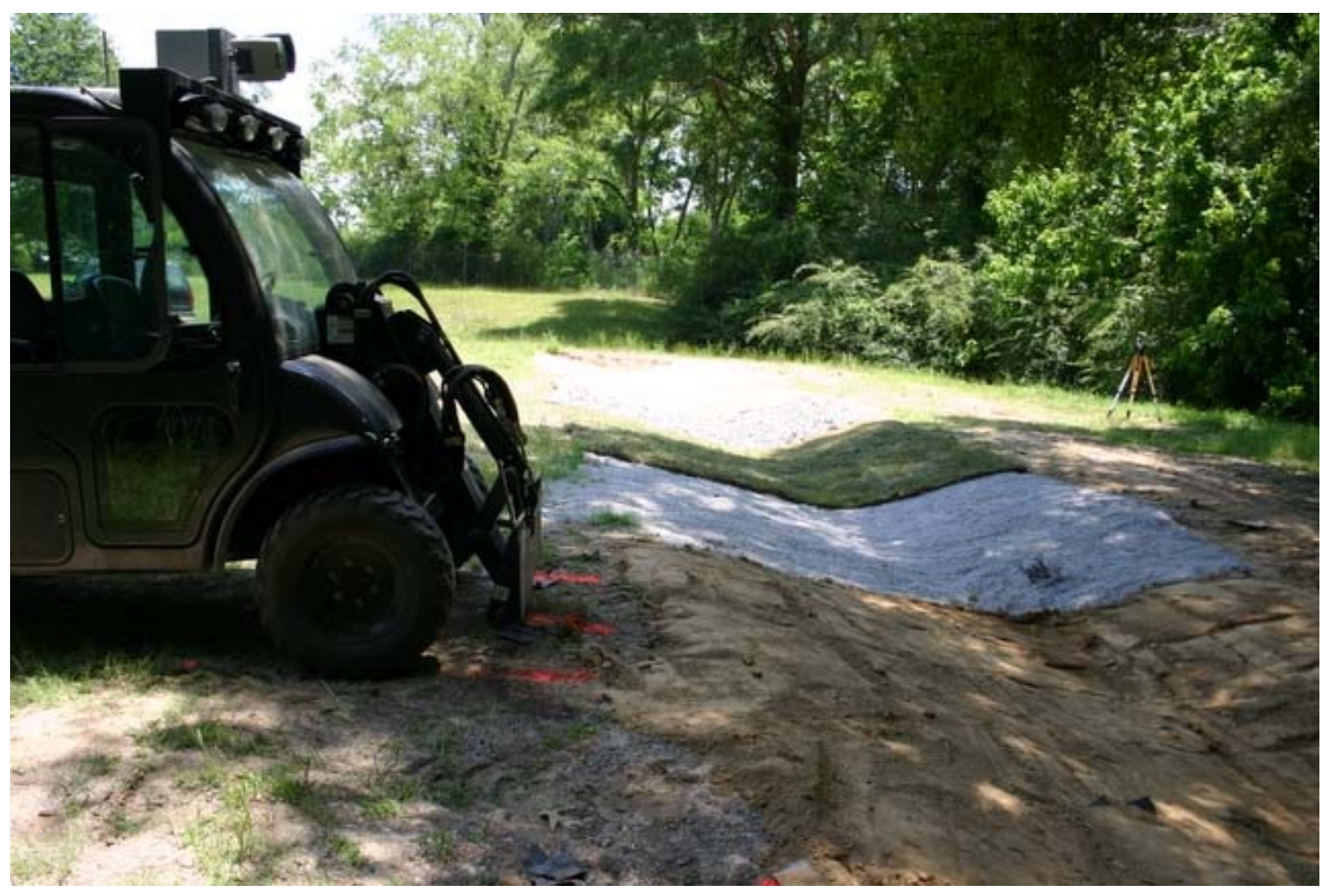

Photo 10. Surface material testing in shade. 


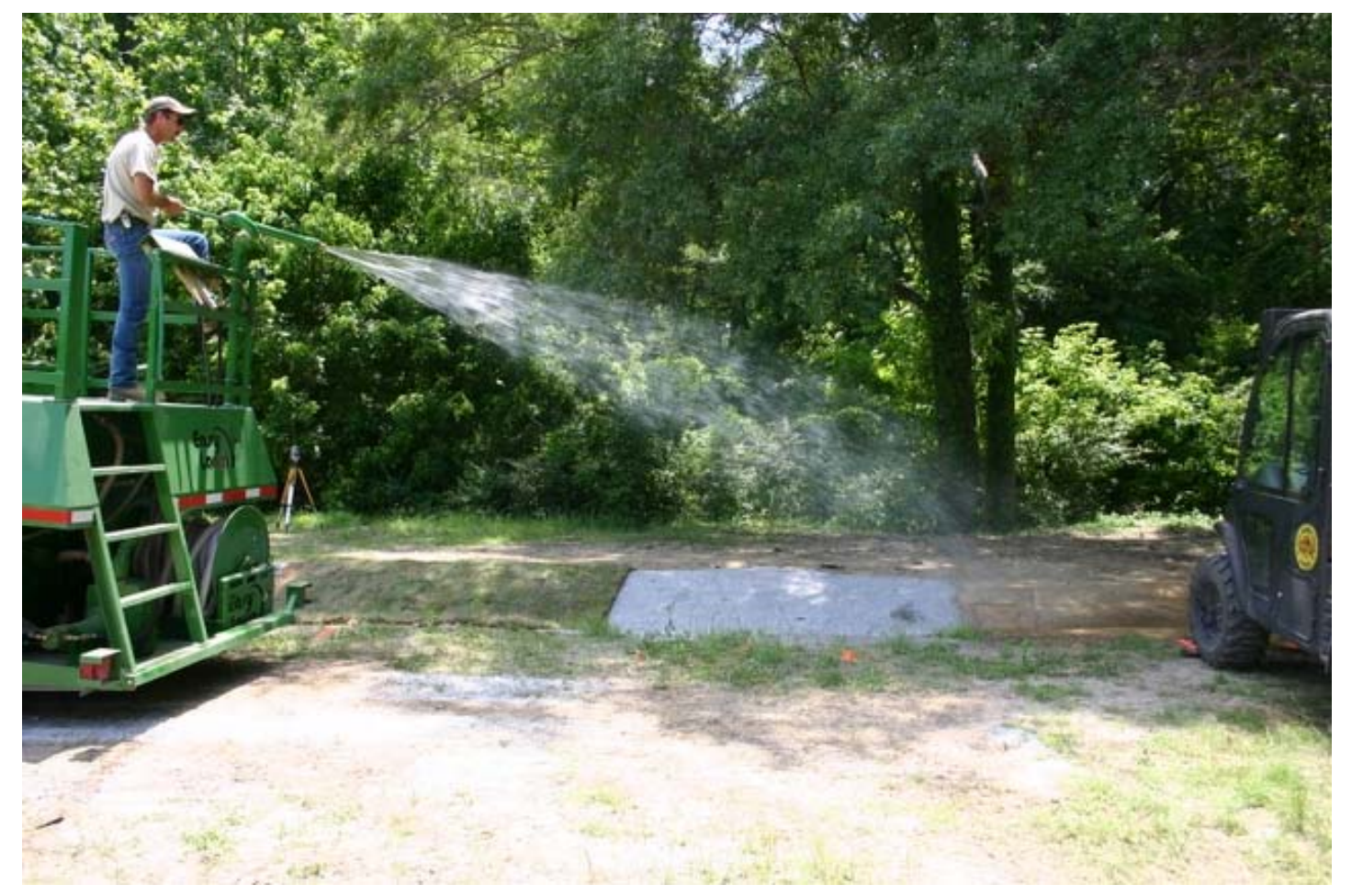

Photo 11. Application of water for the mud surface evaluation.

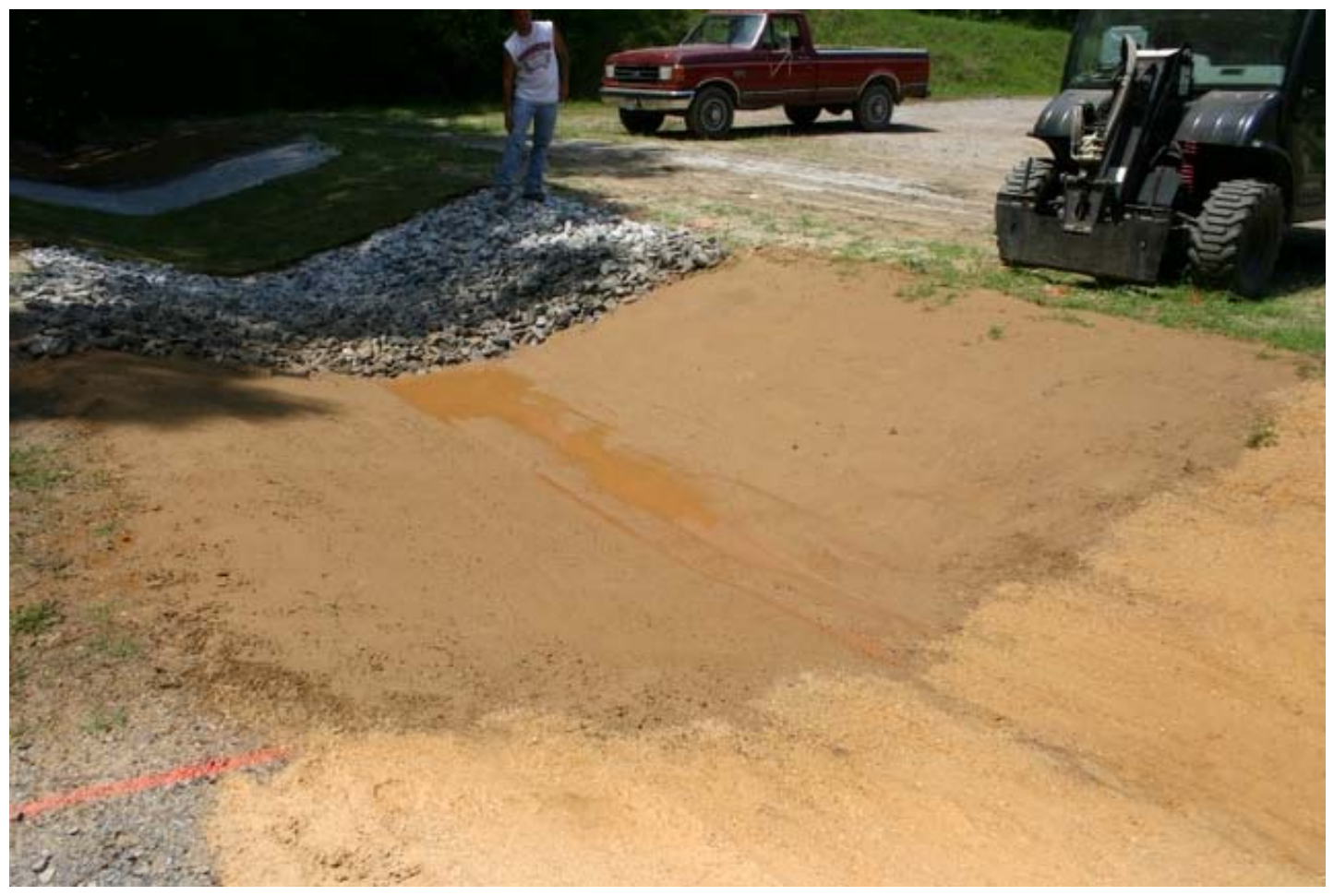

Photo 12. Mud (wet soil) in sun. 


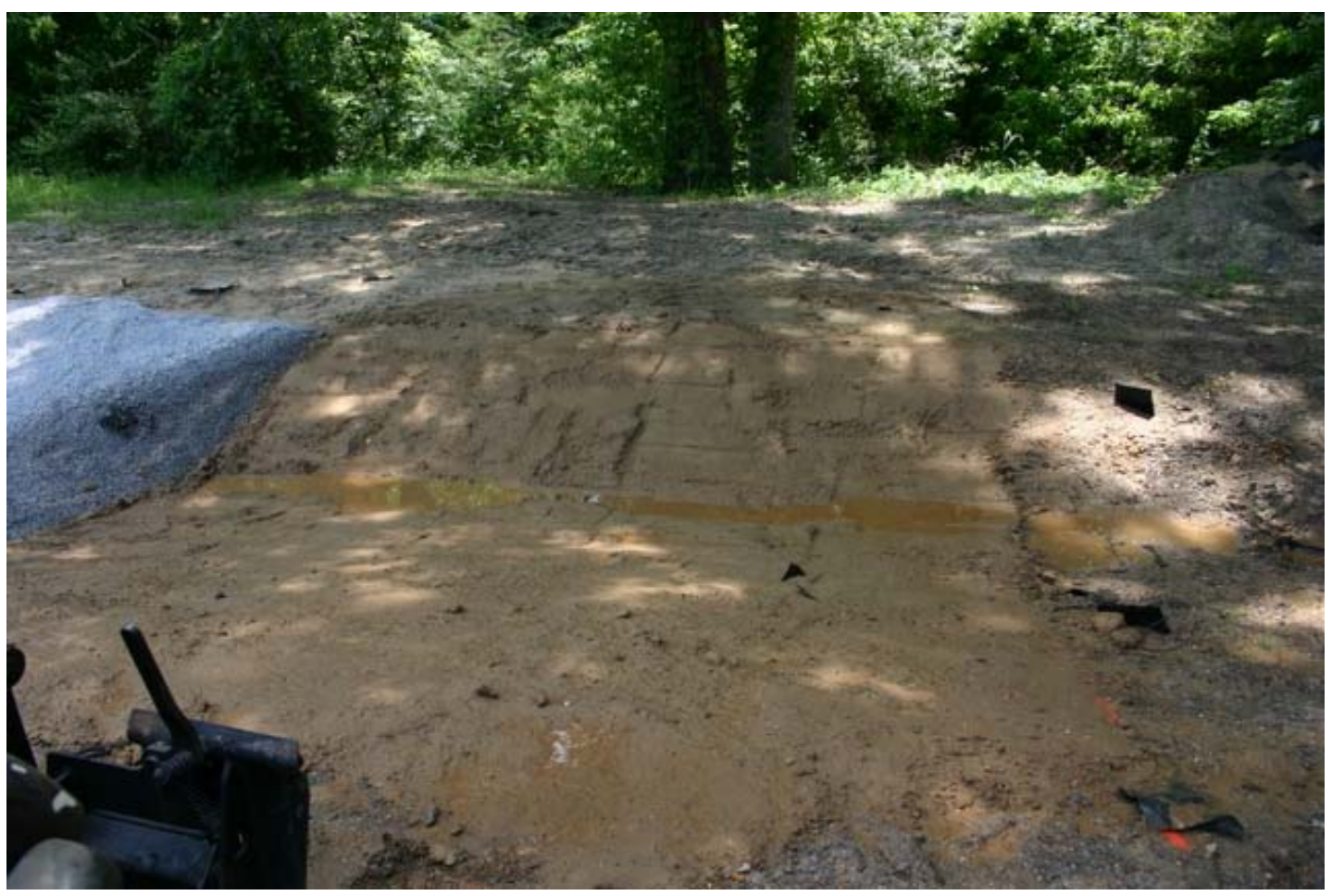

Photo 13. Mud (wet soil) in shade.

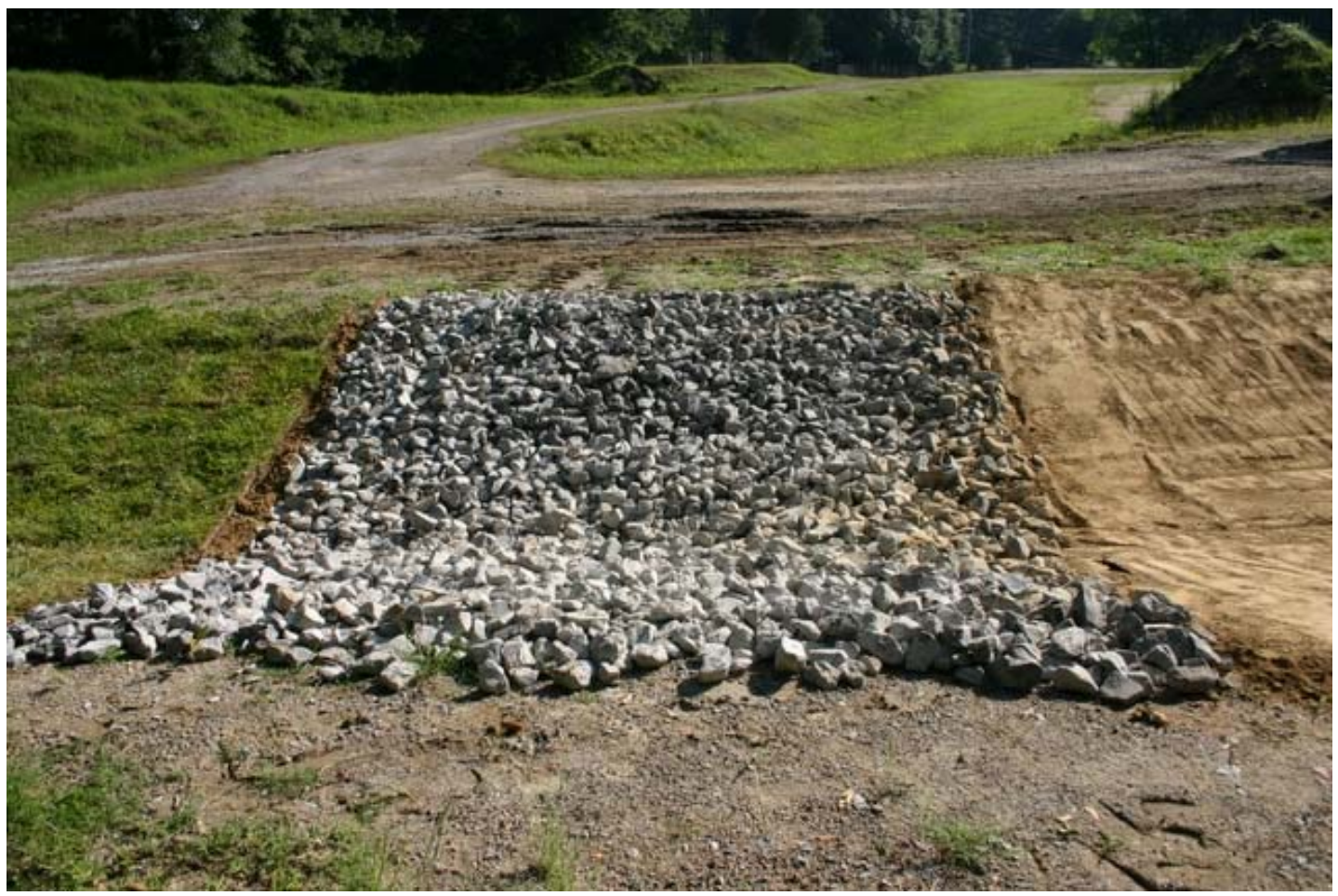

Photo 14. Riprap in sun. 


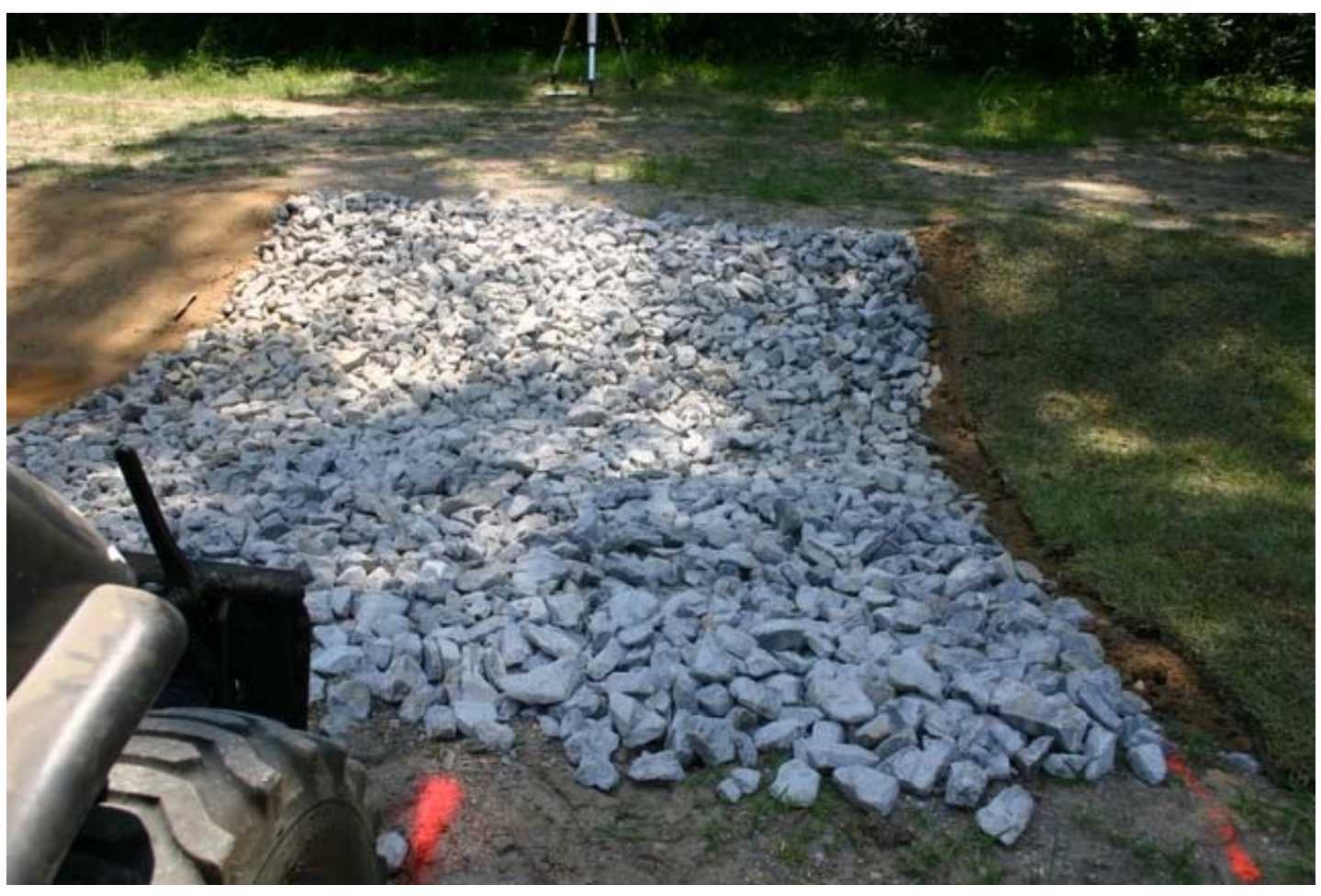

Photo 15. Riprap in shade.

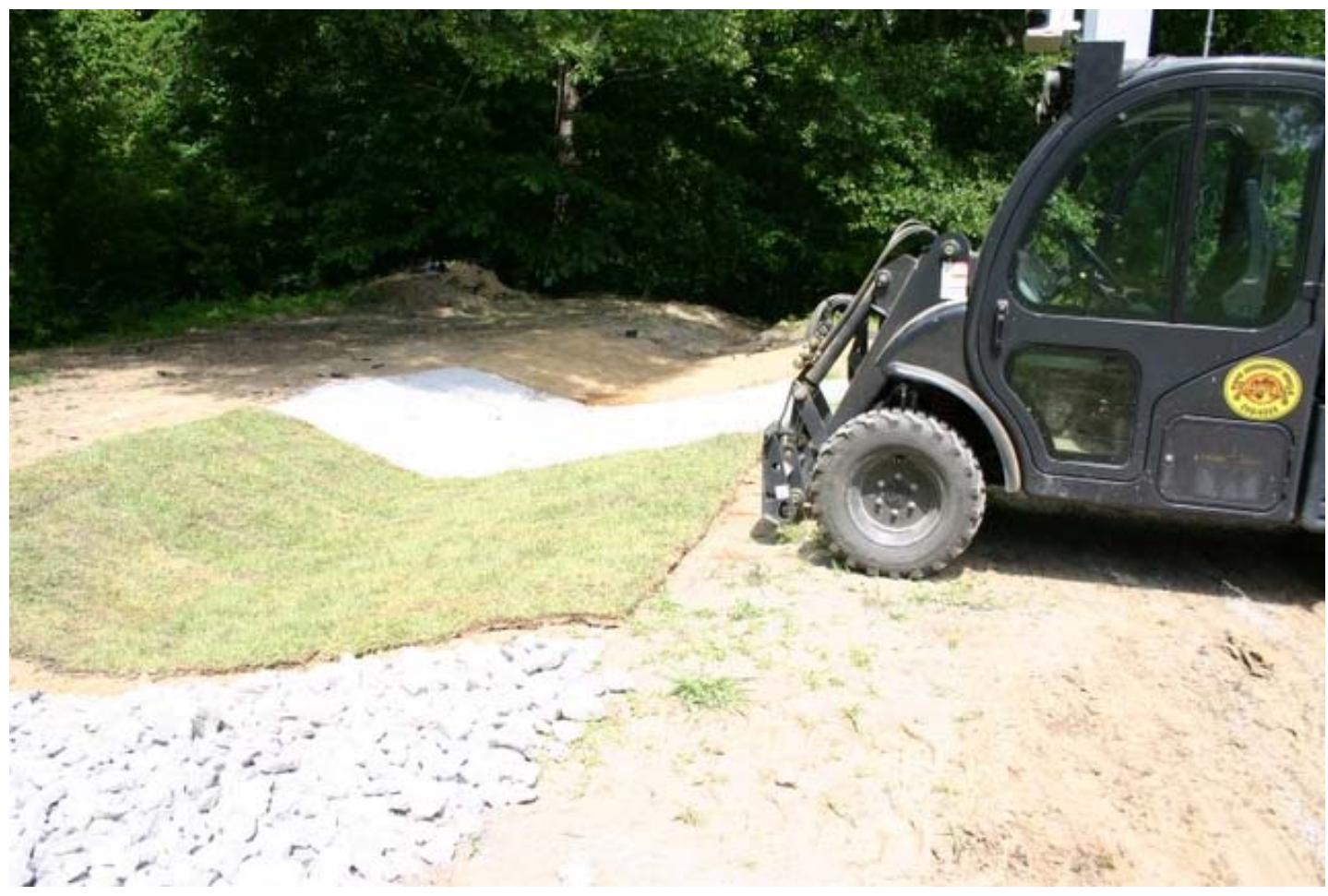

Photo 16. Grass in sun. 


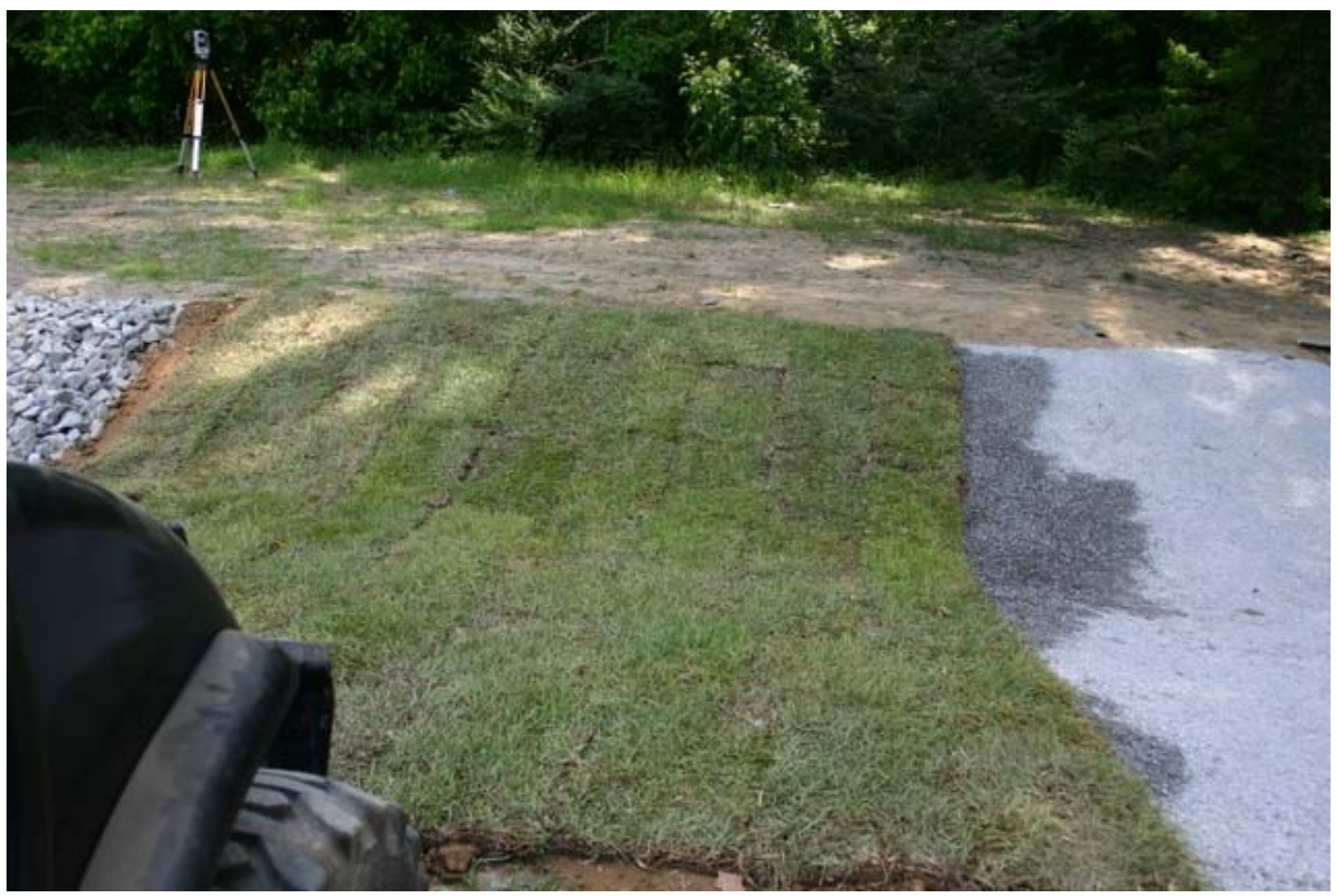

Photo 17. Grass in shade.

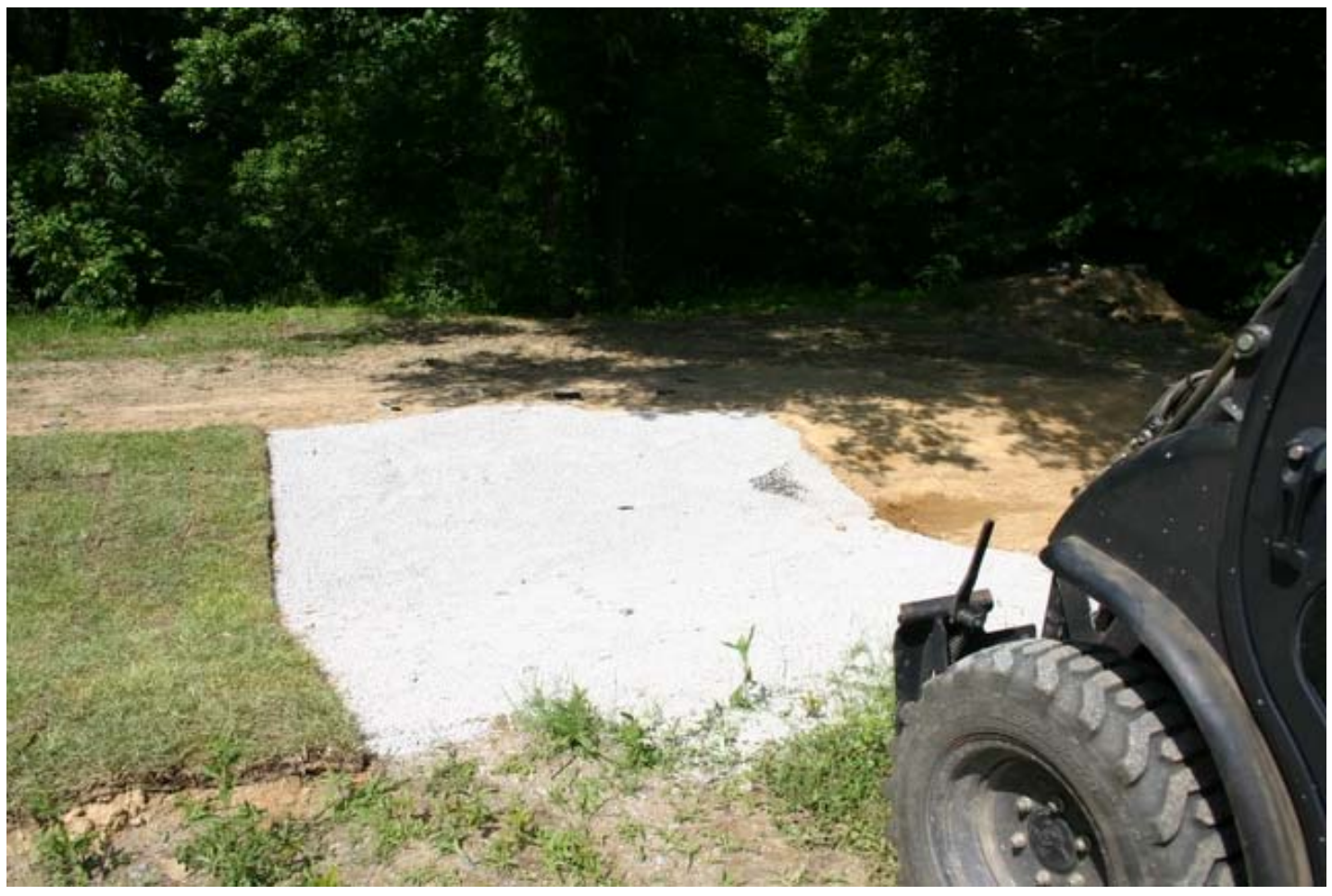

Photo 18. Gravel in sun. 


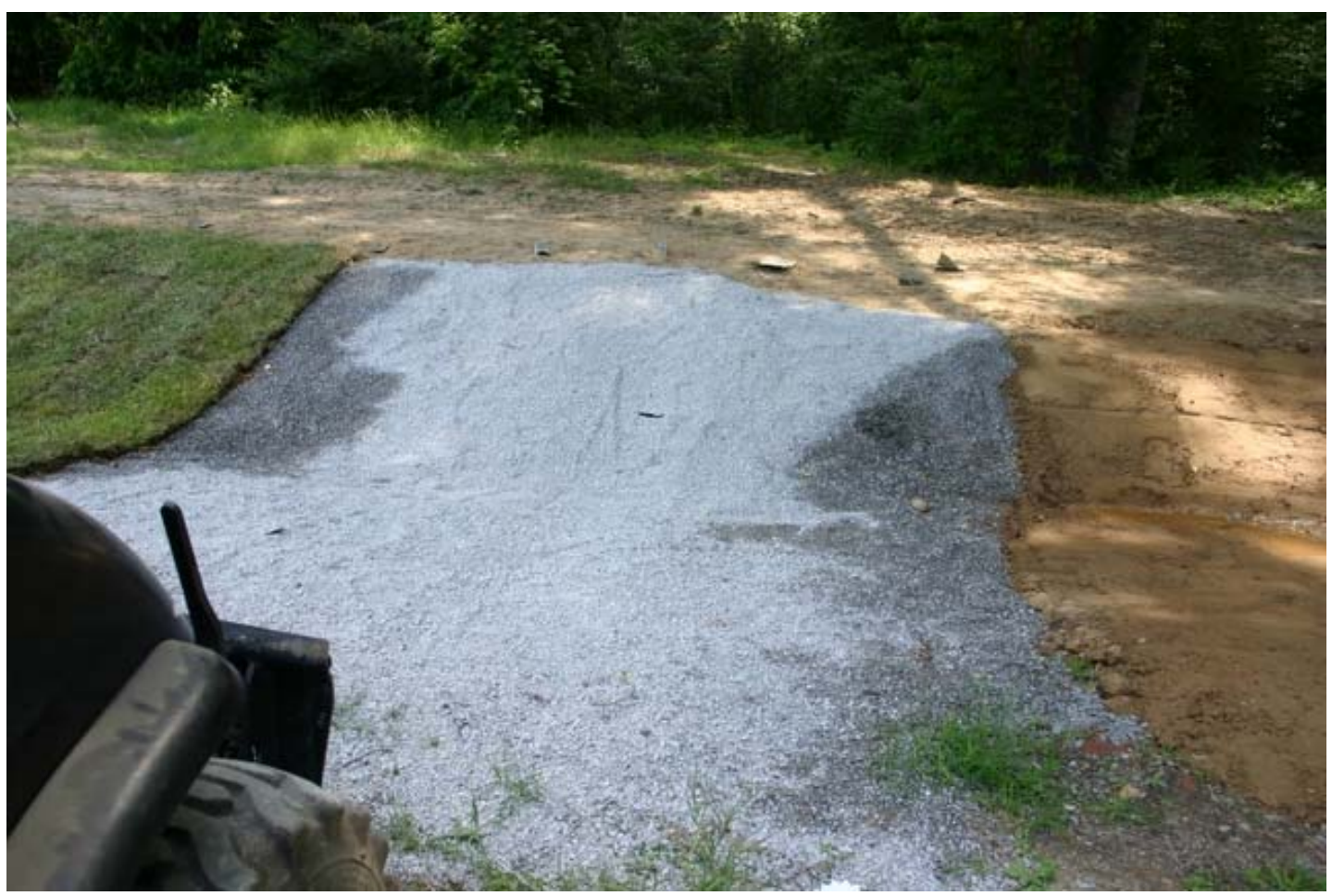

Photo 19. Gravel in shade.

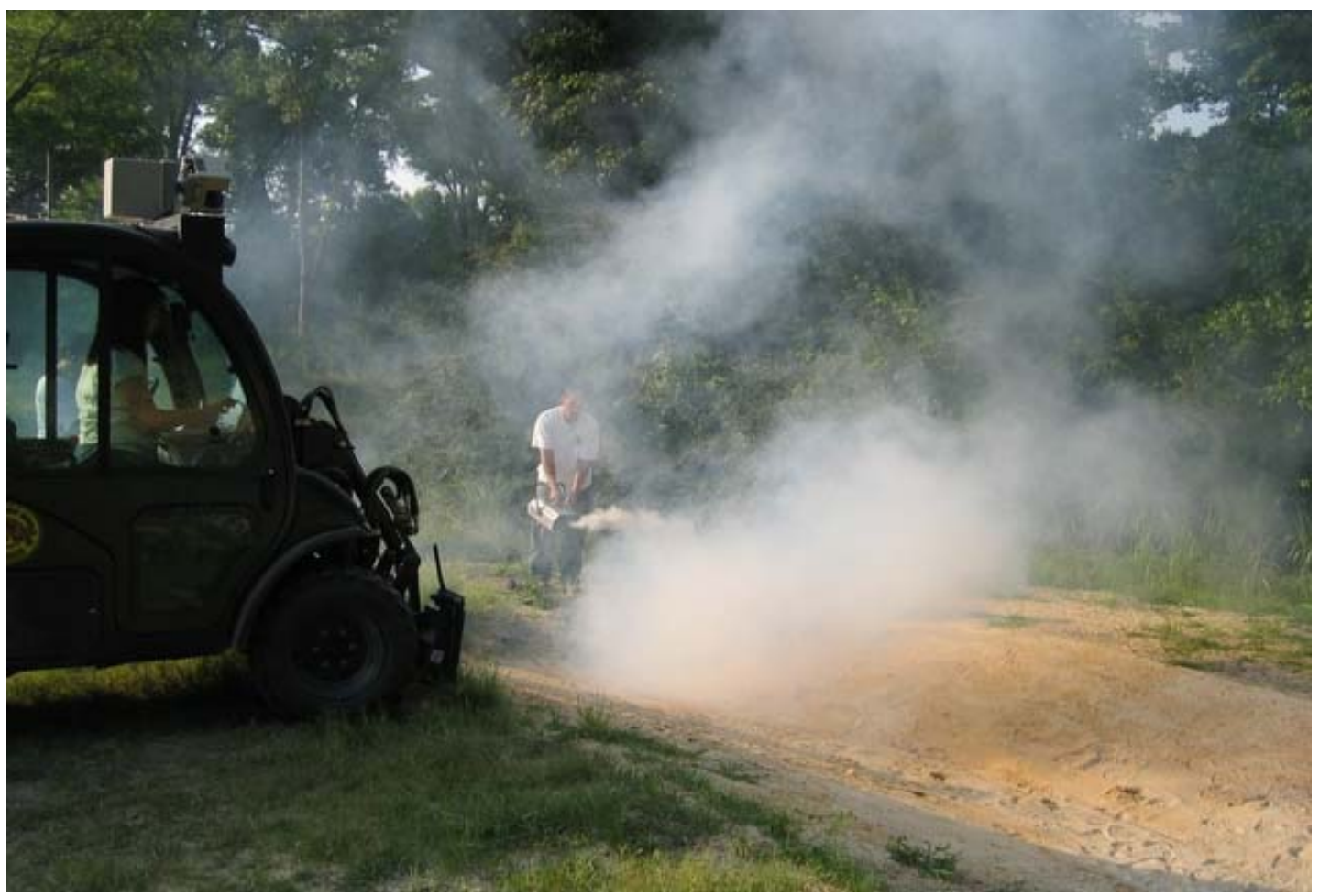

Photo 20. Creating simulated fog/smoke at the sand gap. 


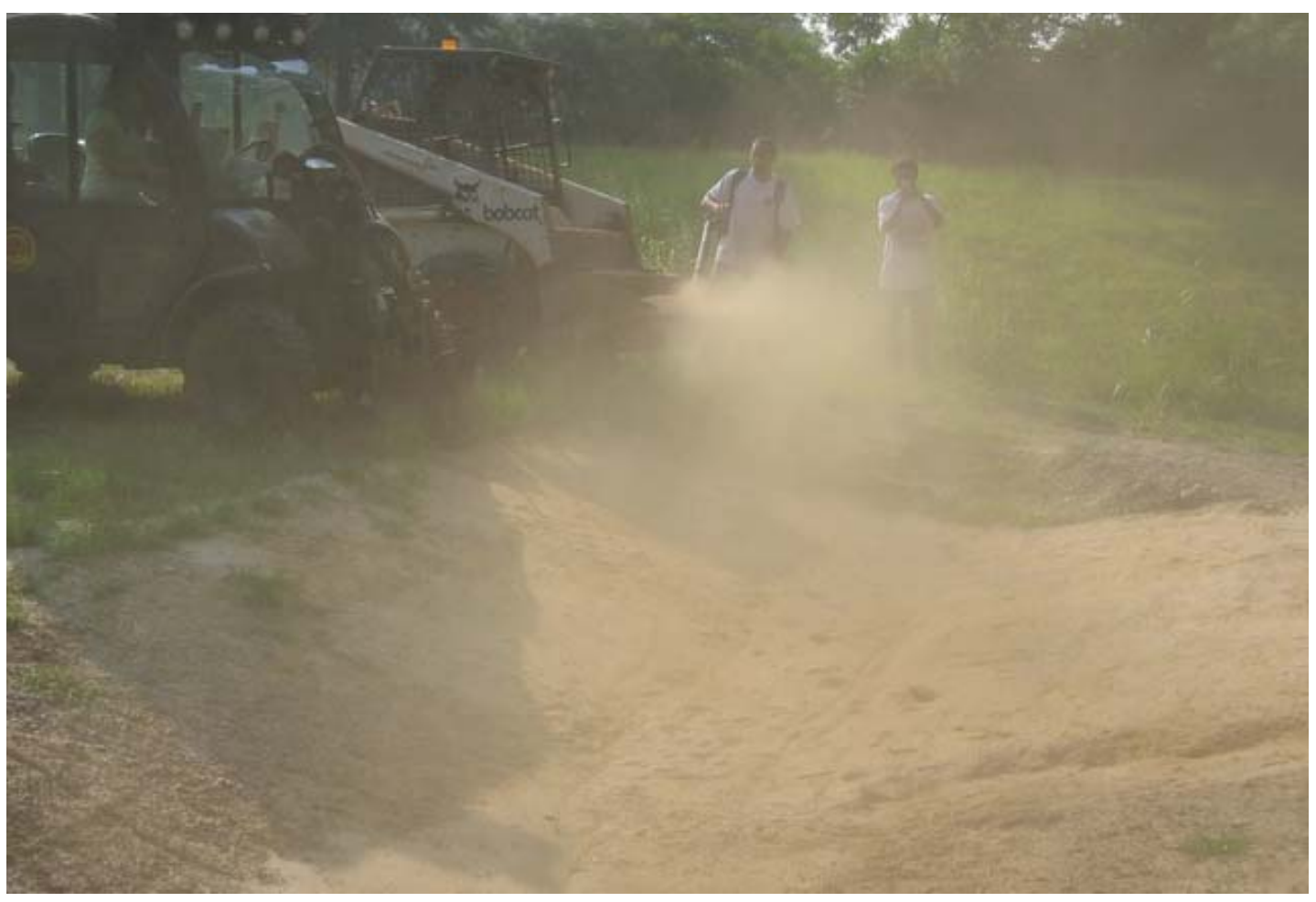

Photo 21. Creating simulated dust with a leaf blower at the sand gap. 


\section{Appendix A Summary of Results}




\section{Table A1}

3D Volume Difference of LADAR and Total Station Data Sets

\begin{tabular}{|c|c|c|c|c|c|c|c|c|c|c|c|c|c|c|c|c|c|c|}
\hline \multicolumn{5}{|c|}{ Gap Properties } & \multicolumn{4}{|c|}{ LASER A } & \multicolumn{4}{|c|}{ LASER B } & \multicolumn{4}{|c|}{ LASER C } & \multicolumn{2}{|c|}{ SUMMARY } \\
\hline GAP & $\begin{array}{c}\text { Stand- } \\
\text { off } \\
(\mathrm{m})\end{array}$ & $\begin{array}{c}\text { Ap- } \\
\text { proach } \\
\text { Slope }\end{array}$ & $\begin{array}{c}\text { Departure } \\
\text { Slope }\end{array}$ & \begin{tabular}{c|} 
No. of \\
Total \\
Station \\
Points \\
\end{tabular} & $\begin{array}{l}\text { No. of } \\
\text { Laser } \\
\text { Points }\end{array}$ & $\begin{array}{l}\mathrm{Cut}^{1} \\
\left(\mathrm{~m}^{3}\right)\end{array}$ & $\begin{array}{l}\text { Fill }^{2} \\
\left(\mathrm{~m}^{3}\right)\end{array}$ & $\begin{array}{c}\text { Total }^{3} \\
\left(\mathrm{~m}^{3}\right)\end{array}$ & $\begin{array}{l}\text { No. of } \\
\text { Laser } \\
\text { Points }\end{array}$ & $\begin{array}{l}\mathrm{Cut}^{1} \\
\left(\mathrm{~m}^{3}\right)\end{array}$ & $\begin{array}{l}\text { Fill }^{2} \\
\left(m^{3}\right)\end{array}$ & $\begin{array}{c}\text { Total }^{3} \\
\left(\mathrm{~m}^{3}\right)\end{array}$ & $\begin{array}{l}\text { No. of } \\
\text { Laser } \\
\text { Points }\end{array}$ & $\begin{array}{l}\text { Cut }^{1} \\
\left(\mathrm{~m}^{3}\right)\end{array}$ & $\begin{array}{l}\text { Fill }^{2} \\
\left(\mathrm{~m}^{3}\right)\end{array}$ & $\begin{array}{c}\text { Total }^{3} \\
\left(\mathrm{~m}^{3}\right)\end{array}$ & $\begin{array}{c}\text { Average } \\
\text { of } \\
\text { Totals } \\
\left(\mathrm{m}^{3}\right)\end{array}$ & $\begin{array}{c}\begin{array}{c}\text { Stdev } \\
\text { of } \\
\text { Totals } \\
\left(\mathrm{m}^{3}\right)\end{array} \\
\end{array}$ \\
\hline A & 0 & $5^{\circ}$ & $10^{\circ}$ & 196 & 16,481 & 0.1 & 0.5 & 0.6 & 16,442 & 0.1 & 0.5 & 0.6 & 16,432 & 0.2 & 0.5 & 0.7 & 0.63 & 0.06 \\
\hline A & 0 & $10^{\circ}$ & $5^{\circ}$ & 173 & 13,204 & 0.1 & 0.5 & 0.6 & 13,537 & 0.1 & 0.5 & 0.6 & 13,546 & 0.1 & 0.5 & 0.6 & 0.60 & 0.00 \\
\hline$B$ & 0 & $15^{\circ}$ & $20^{\circ}$ & 208 & 16,683 & 0.1 & 0.9 & 1 & 16,595 & 0.1 & 0.8 & 0.9 & 16,381 & 0.1 & 0.8 & 0.9 & 0.93 & .0 \\
\hline$B$ & 1.5 & $15^{\circ}$ & $20^{\circ}$ & 209 & 16,483 & 0.1 & 0.7 & 0.8 & 16,422 & 0.1 & 0.7 & 0.8 & 16,069 & 0.1 & 0.7 & 0.8 & 0.80 & 0.00 \\
\hline$B$ & 0 & $20^{\circ}$ & $15^{\circ}$ & 207 & 16,716 & 0.1 & 0.9 & 1 & 9 & 0 & 0.9 & 0.9 & 45 & 0.1 & 0.9 & 1 & 0.97 & 0.06 \\
\hline$B$ & 1.5 & $20^{\circ}$ & $15^{\circ}$ & 209 & 15,844 & 0 & 1.9 & 1.9 & 15,834 & 0 & 1.9 & 1.9 & 14,354 & 0 & 2 & 2 & 1.93 & 0.06 \\
\hline $\mathrm{C}$ & 0 & $30^{\circ}$ & $40^{\circ}$ & 279 & 21,607 & 0.4 & 0.9 & 1.3 & 21,621 & 0.4 & 0.8 & 1.2 & 21,648 & 0.4 & 0.8 & 1.2 & 1.23 & 0.06 \\
\hline$C$ & 1.5 & $30^{\circ}$ & $40^{\circ}$ & 280 & 19,225 & 0.2 & 1.7 & 1.9 & 20,490 & 0.2 & 1.6 & 1.8 & 19,945 & 0.2 & 1.6 & 1.8 & 1.83 & 0.06 \\
\hline$C$ & 0 & $40^{\circ}$ & $30^{\circ}$ & 280 & 22,137 & 0.3 & 1 & 1.3 & $n / a^{6}$ & $n / a^{6}$ & $n / a^{6}$ & $n / a^{6}$ & 21,330 & 0.3 & 1.1 & 1.4 & 1.35 & 0.07 \\
\hline $\mathrm{C}$ & 1.5 & $40^{\circ}$ & $30^{\circ}$ & 280 & 18,253 & 0.2 & 2.1 & 2.3 & 18,187 & 0.2 & 2.1 & 2.3 & 17,254 & 0.2 & 2.2 & 2.4 & 2.33 & 0.06 \\
\hline D & 0 & $45^{\circ}$ & $90^{\circ}$ & 174 & 16,904 & 0.7 & 2.7 & 3.4 & 15,679 & 0.7 & 2.8 & 3.5 & 16,599 & 0.7 & 2.7 & 3.4 & 3.43 & 6 \\
\hline D & 1.5 & $45^{\circ}$ & $90^{\circ}$ & 175 & 11,445 & 0.6 & 6.1 & 6.7 & 11,528 & 0.7 & 6 & 6.7 & 11,346 & 0.6 & 6.1 & 6.7 & 70 & .00 \\
\hline$D$ & 0 & $90^{\circ}$ & $45^{\circ}$ & 175 & 14,905 & 0.3 & 8.5 & 8.8 & 15,267 & 0.3 & 8.5 & 8.8 & 15,006 & 0.3 & 8.6 & 8.9 & 8.83 & 0.06 \\
\hline$D$ & 1.5 & $90^{\circ}$ & $45^{\circ}$ & 175 & 12,499 & 0.3 & 15.6 & 15.9 & 12,509 & 0.3 & 15.6 & 15.9 & 12,402 & 0.2 & 14.5 & 14.7 & 15.50 & 0.69 \\
\hline
\end{tabular}

${ }^{1}$ Cut: Volume of LADAR data greater than total station data.

${ }^{2}$ Fill: Volume of LADAR data less than total station data.

${ }^{3}$ Total: Sum of differences between the total station and LADAR data sets.

${ }^{4}$ Average of Totals: Average of Laser A, Laser B, and Laser C Total values.

${ }^{5}$ Stdev of Totals: Standard deviation of Laser A, Laser B, and Laser C Total Values.

${ }^{6}$ Data collected were corrupted. 


\begin{tabular}{|c|c|c|c|c|c|c|c|c|c|c|c|c|c|c|c|}
\hline \multicolumn{16}{|c|}{$\begin{array}{l}\text { Table A2 } \\
\text { Error for Driver Side Profile }\end{array}$} \\
\hline \multicolumn{4}{|c|}{ Gap Properties } & \multicolumn{4}{|c|}{$\begin{array}{c}\text { TOTAL } \\
\text { (Average error }{ }^{1} \text { for entire gap) }\end{array}$} & \multicolumn{4}{|c|}{$\begin{array}{c}\text { APPROACH } \\
\text { (Average error }{ }^{1} \text { for approach slope) } \\
\end{array}$} & \multicolumn{4}{|c|}{$\begin{array}{c}\text { DEPARTURE } \\
\text { (Average error }{ }^{1} \text { for departure slope) }\end{array}$} \\
\hline GAP & $\begin{array}{l}\text { Stand- } \\
\text { off }(m)\end{array}$ & $\begin{array}{l}\text { Approach } \\
\text { Slope }\end{array}$ & \begin{tabular}{|} 
Departure \\
Slope
\end{tabular} & $\begin{array}{c}\text { Laser } \\
\mathrm{A} \\
(\mathrm{cm})\end{array}$ & $\begin{array}{c}\text { Laser } \\
\text { B } \\
(\mathrm{cm})\end{array}$ & $\begin{array}{l}\text { Laser } \\
\text { C } \\
(\mathrm{cm})\end{array}$ & $\begin{array}{l}\text { AVG } \\
(\mathrm{cm})\end{array}$ & $\begin{array}{c}\text { Laser } \\
\text { A } \\
(\mathrm{cm}) \\
\end{array}$ & $\begin{array}{c}\text { Laser } \\
\text { B } \\
(\mathrm{cm})\end{array}$ & $\begin{array}{c}\text { Laser } \\
\mathbf{C} \\
(\mathrm{cm})\end{array}$ & $\begin{array}{l}\text { AVG } \\
\text { (cm) }\end{array}$ & $\begin{array}{l}\text { Laser } \\
\text { A } \\
(\mathrm{cm})\end{array}$ & $\begin{array}{l}\text { Laser } \\
\text { B } \\
\text { (cm) }\end{array}$ & $\begin{array}{l}\text { Laser } \\
\text { C } \\
\text { (cm) }\end{array}$ & $\begin{array}{l}\text { AVG } \\
(\mathrm{cm})\end{array}$ \\
\hline A & 0 & $5^{\circ}$ & $10^{\circ}$ & 0.64 & 0.43 & 0.79 & 0.62 & 0.80 & 0.60 & 0.80 & 0.73 & 0.25 & 0.00 & 0.75 & 0.33 \\
\hline A & 0 & $10^{\circ}$ & $5^{\circ}$ & 1.46 & 1.23 & 1.62 & 1.44 & 2.00 & 1.25 & 1.75 & 1.67 & 1.22 & 1.22 & 1.56 & 1.33 \\
\hline B & 0 & $15^{\circ}$ & $20^{\circ}$ & 3.00 & 2.46 & 3.00 & 2.82 & 3.86 & 3.14 & 4.14 & 3.71 & 2.00 & 1.67 & 1.67 & 1.78 \\
\hline$B$ & 1.5 & $15^{\circ}$ & $20^{\circ}$ & 2.80 & 2.53 & 3.20 & 2.84 & 3.78 & 3.00 & 4.00 & 3.59 & 1.33 & 1.83 & 2.00 & 1.72 \\
\hline$B$ & 0 & $20^{\circ}$ & $15^{\circ}$ & 1.93 & 1.71 & 1.64 & 1.76 & 1.50 & 0.67 & 1.33 & 1.17 & 2.25 & 2.50 & 1.88 & 2.21 \\
\hline B & 1.5 & $20^{\circ}$ & $15^{\circ}$ & 6.00 & 5.87 & 6.80 & 6.22 & 4.57 & 4.86 & 5.57 & 5.00 & 7.25 & 6.75 & 7.88 & 7.29 \\
\hline $\mathrm{C}$ & 0 & $30^{\circ}$ & $40^{\circ}$ & 2.79 & 3.16 & 2.89 & 2.95 & 2.27 & 3.55 & 3.09 & 2.97 & 3.50 & 2.63 & 2.62 & 2.92 \\
\hline $\mathrm{C}$ & 1.5 & $30^{\circ}$ & $40^{\circ}$ & 5.63 & 5.37 & 4.74 & 5.25 & 7.64 & 7.27 & 6.73 & 7.21 & 2.88 & 2.75 & 2.00 & 2.54 \\
\hline C & 0 & $40^{\circ}$ & $30^{\circ}$ & 1.42 & $\mathrm{n} / \mathrm{a}^{2}$ & 1.68 & 1.55 & 1.56 & $\mathrm{n} / \mathrm{a}^{2}$ & 2.11 & 1.83 & 1.30 & $\mathrm{n} / \mathrm{a}^{2}$ & 1.30 & 1.30 \\
\hline C & 1.5 & $40^{\circ}$ & $30^{\circ}$ & 6.16 & 6.74 & 7.16 & 6.68 & 7.22 & 7.67 & 8.78 & 7.89 & 5.20 & 5.90 & 5.70 & 5.60 \\
\hline$D$ & 0 & $45^{\circ}$ & $90^{\circ}$ & 7.56 & 8.06 & 8.06 & 7.89 & 8.60 & 9.60 & 8.30 & 8.83 & 6.25 & 6.12 & 7.75 & 6.71 \\
\hline D & 1.5 & $45^{\circ}$ & $90^{\circ}$ & 26.21 & 26.00 & 25.68 & 25.96 & 33.42 & 32.08 & 32.50 & 32.67 & 13.86 & 15.57 & 14.00 & 14.48 \\
\hline D & 0 & $90^{\circ}$ & $45^{\circ}$ & 27.59 & 27.59 & 27.94 & 27.71 & 64.43 & 64.00 & 64.86 & 64.43 & 1.80 & 2.10 & 2.10 & 2.00 \\
\hline D & 1.5 & $90^{\circ}$ & $45^{\circ}$ & $\mid 71.88$ & 72.00 & 64.47 & 69.45 & 109.00 & 109.00 & 97.09 & 105.03 & 3.83 & 4.17 & 4.67 & 4.22 \\
\hline
\end{tabular}




\begin{tabular}{|c|c|c|c|c|c|c|c|c|c|c|c|c|c|c|c|}
\hline \multicolumn{16}{|c|}{$\begin{array}{l}\text { Table A3 } \\
\text { Error for Center Profile }\end{array}$} \\
\hline \multicolumn{4}{|c|}{ Gap Properties } & \multicolumn{4}{|c|}{$\begin{array}{c}\text { TOTAL } \\
\text { (Average error }{ }^{1} \text { for entire gap) } \\
\end{array}$} & \multicolumn{4}{|c|}{$\begin{array}{c}\text { APPROACH } \\
\text { (Average error }{ }^{\text {for approach slope) }} \\
\end{array}$} & \multicolumn{4}{|c|}{\begin{tabular}{|c} 
DEPARTURE \\
(Average error ${ }^{1}$ for departure slope) \\
\end{tabular}} \\
\hline GAP & \begin{tabular}{|l|} 
Stand- \\
off $(m)$
\end{tabular} & $\begin{array}{c}\text { Approach } \\
\text { Slope }\end{array}$ & $\begin{array}{c}\text { Departure } \\
\text { Slope }\end{array}$ & $\begin{array}{c}\text { Laser } \\
\text { A } \\
(\mathrm{cm})\end{array}$ & $\begin{array}{l}\text { Laser } \\
\text { B } \\
(\mathrm{cm})\end{array}$ & $\begin{array}{l}\text { Laser } \\
\text { C } \\
\text { (cm) }\end{array}$ & $\begin{array}{l}\text { AVG } \\
(\mathbf{c m})\end{array}$ & \begin{tabular}{|c} 
Laser \\
A \\
$(\mathrm{cm})$
\end{tabular} & $\begin{array}{l}\text { Laser } \\
\text { B } \\
(\mathrm{cm})\end{array}$ & \begin{tabular}{|c} 
Laser \\
C \\
$(\mathrm{cm})$
\end{tabular} & $\begin{array}{l}\text { AVG } \\
(\mathrm{cm})\end{array}$ & $\mid$\begin{tabular}{|c|} 
Laser \\
$A$ \\
$(\mathrm{~cm})$
\end{tabular} & \begin{tabular}{|c|} 
Laser \\
$B$ \\
$(\mathrm{~cm})$
\end{tabular} & $\begin{array}{l}\text { Laser } \\
\text { C } \\
(\mathrm{cm})\end{array}$ & $\begin{array}{l}\text { AVG } \\
(\mathbf{c m})\end{array}$ \\
\hline A & 0 & $5^{\circ}$ & $10^{\circ}$ & 2.00 & 1.92 & 1.67 & 1.86 & 2.00 & 2.00 & 1.63 & 1.88 & 2.00 & 1.75 & 1.75 & 1.83 \\
\hline A & 0 & $10^{\circ}$ & $5^{\circ}$ & 2.46 & 3.23 & 2.23 & 2.64 & 1.50 & 3.50 & 2.25 & 2.42 & 2.89 & 3.11 & 2.22 & 2.74 \\
\hline $\mathrm{B}$ & 0 & $15^{\circ}$ & $20^{\circ}$ & 5.08 & 4.92 & 5.00 & 5.00 & 6.43 & 6.14 & 6.00 & 6.19 & 3.50 & 3.50 & 3.83 & 3.61 \\
\hline B & 1.5 & $15^{\circ}$ & $20^{\circ}$ & 3.87 & 3.93 & 4.53 & 4.11 & 4.78 & 4.67 & 5.33 & 4.93 & 2.50 & 2.83 & 3.33 & 2.89 \\
\hline $\mathrm{B}$ & 0 & $20^{\circ}$ & $15^{\circ}$ & 4.85 & 4.69 & 4.23 & 4.59 & 3.80 & 4.00 & 3.60 & 3.80 & 5.50 & 5.13 & 4.62 & 5.08 \\
\hline $\mathrm{B}$ & 1.5 & $20^{\circ}$ & $15^{\circ}$ & 9.20 & 9.00 & 10.07 & 9.42 & 9.14 & 8.57 & 9.71 & 9.14 & 9.25 & 9.38 & 10.38 & 9.67 \\
\hline $\mathrm{C}$ & 0 & $30^{\circ}$ & $40^{\circ}$ & 3.33 & 3.06 & 3.33 & 3.24 & 4.10 & 3.60 & 3.80 & 3.83 & 2.38 & 2.38 & 2.75 & 2.50 \\
\hline C & 1.5 & $30^{\circ}$ & $40^{\circ}$ & 6.39 & 5.72 & 6.56 & 6.22 & 9.00 & 8.30 & 7.60 & 8.30 & 3.13 & 2.50 & 5.25 & 3.63 \\
\hline C & 0 & $40^{\circ}$ & $30^{\circ}$ & 3.44 & $\mathrm{n} / \mathrm{a}^{2}$ & 3.72 & 3.58 & 3.62 & $\mathrm{n} / \mathrm{a}^{2}$ & 3.75 & 3.69 & 3.30 & $\mathrm{n} / \mathrm{a}^{2}$ & 3.70 & 3.50 \\
\hline C & 1.5 & $40^{\circ}$ & $30^{\circ}$ & 8.95 & 9.16 & 7.89 & 8.67 & 9.78 & 9.56 & 8.89 & 9.41 & 8.20 & 8.80 & 7.00 & 8.00 \\
\hline $\mathrm{D}$ & 0 & $45^{\circ}$ & $90^{\circ}$ & 10.22 & 9.28 & 9.83 & 9.78 & 11.91 & 10.45 & 11.09 & 11.15 & 7.57 & 7.43 & 7.86 & 7.62 \\
\hline D & 1.5 & $45^{\circ}$ & $90^{\circ}$ & 27.78 & 27.50 & 28.17 & 27.81 & 36.18 & 35.27 & 36.73 & 36.06 & 14.57 & 15.29 & 14.71 & 14.86 \\
\hline $\mathrm{D}$ & 0 & $90^{\circ}$ & $45^{\circ}$ & 41.65 & 40.59 & 41.88 & 41.37 & 98.57 & 95.29 & 97.71 & 97.19 & 1.80 & 2.30 & 2.80 & 2.30 \\
\hline$D$ & 1.5 & $90^{\circ}$ & $45^{\circ}$ & 80.16 & 80.16 & 80.63 & 80.32 & $\mid$\begin{tabular}{||c}
124.08 \\
\end{tabular} & 124.17 & 124.83 & 124.36 & 4.86 & 4.71 & 4.86 & 4.81 \\
\hline
\end{tabular}




\begin{tabular}{|c|c|c|c|c|c|c|c|c|c|c|c|c|c|c|c|}
\hline \multicolumn{16}{|c|}{$\begin{array}{l}\text { Table A4 } \\
\text { Error for Passenger Side Profile }\end{array}$} \\
\hline \multicolumn{4}{|c|}{ Gap Properties } & \multicolumn{4}{|c|}{$\begin{array}{c}\text { TOTAL } \\
\text { (Average error }{ }^{1} \text { for entire gap) }\end{array}$} & \multicolumn{4}{|c|}{\begin{tabular}{|c||} 
APPROACH \\
(Average error ${ }^{1}$ for approach slope) \\
\end{tabular}} & \multicolumn{4}{|c|}{$\begin{array}{c}\text { DEPARTURE } \\
\text { (Average error }{ }^{1} \text { for departure slope) }\end{array}$} \\
\hline GAP & \begin{tabular}{|c|}
$\begin{array}{c}\text { Stand- } \\
\text { off } \\
(\mathrm{m})\end{array}$ \\
\end{tabular} & $\begin{array}{l}\text { Approach } \\
\text { Slope }\end{array}$ & $\begin{array}{c}\text { Departure } \\
\text { Slope }\end{array}$ & $\mid \begin{array}{c}\text { Laser } \\
\text { A } \\
(\mathrm{cm})\end{array}$ & $\begin{array}{l}\text { Laser } \\
\text { B } \\
(\mathrm{cm})\end{array}$ & $\begin{array}{l}\text { Laser } \\
\text { C } \\
(\mathrm{cm})\end{array}$ & $\begin{array}{l}\text { AVG } \\
(\mathrm{cm})\end{array}$ & \begin{tabular}{|c} 
Laser \\
A \\
$(\mathrm{cm})$
\end{tabular} & \begin{tabular}{|c|} 
Laser \\
$\mathrm{B}$ \\
$(\mathrm{cm})$
\end{tabular} & \begin{tabular}{|c} 
Laser \\
C \\
$(\mathrm{cm})$
\end{tabular} & $\begin{array}{l}\text { AVG } \\
(\mathbf{c m})\end{array}$ & \begin{tabular}{|c|} 
Laser \\
$\mathrm{A}$ \\
$(\mathrm{cm})$
\end{tabular} & \begin{tabular}{|c|} 
Laser \\
B \\
$(\mathrm{cm})$
\end{tabular} & $\begin{array}{l}\text { Laser } \\
\text { C } \\
(\mathrm{cm})\end{array}$ & $\begin{array}{l}\text { AVG } \\
(\mathrm{cm})\end{array}$ \\
\hline$A$ & 0 & $5^{\circ}$ & $10^{\circ}$ & 4.42 & 5.25 & 4.75 & 4.81 & 4.56 & 4.89 & 4.78 & 4.74 & 4.00 & 6.33 & 4.67 & 5.00 \\
\hline A & 0 & $10^{\circ}$ & $5^{\circ}$ & 4.69 & 5.15 & 4.46 & 4.77 & 3.75 & 4.25 & 3.00 & 3.67 & 5.11 & 5.56 & 5.11 & 5.26 \\
\hline $\mathrm{B}$ & 0 & $15^{\circ}$ & $20^{\circ}$ & 7.15 & 7.15 & 7.00 & 7.10 & 8.14 & 8.14 & 8.29 & 8.19 & 6.00 & 6.00 & 5.50 & 5.83 \\
\hline B & 1.5 & $15^{\circ}$ & $20^{\circ}$ & 7.73 & 7.87 & 8.60 & 8.07 & 8.56 & 8.44 & 9.33 & 8.78 & 6.50 & 7.00 & 7.50 & 7.00 \\
\hline $\mathrm{B}$ & 0 & $20^{\circ}$ & $15^{\circ}$ & 7.15 & 7.38 & 7.15 & 7.23 & 5.40 & 5.40 & 4.60 & 5.13 & 8.25 & 8.63 & 8.75 & 8.54 \\
\hline B & 1.5 & $20^{\circ}$ & $15^{\circ}$ & 12.33 & 12.13 & 13.00 & 12.49 & 10.29 & 10.57 & 11.57 & 10.81 & 14.13 & 13.50 & 14.25 & 13.96 \\
\hline C & 0 & $30^{\circ}$ & $40^{\circ}$ & 4.53 & 4.63 & 4.79 & 4.65 & 5.82 & 5.55 & 5.55 & 5.64 & 2.75 & 3.37 & 3.75 & 3.29 \\
\hline C & 1.5 & $30^{\circ}$ & $40^{\circ}$ & 9.58 & 8.11 & 9.16 & 8.95 & 11.36 & 10.00 & 10.36 & 10.58 & 7.13 & 5.50 & 7.50 & 6.71 \\
\hline$C$ & 0 & $40^{\circ}$ & $30^{\circ}$ & 5.26 & $\mathrm{n} / \mathrm{a}^{2}$ & 6.00 & 5.63 & 5.22 & $\mathrm{n} / \mathrm{a}^{2}$ & 5.89 & 5.56 & 5.30 & $\mathrm{n} / \mathrm{a}^{2}$ & 6.10 & 5.70 \\
\hline$C$ & 1.5 & $40^{\circ}$ & $30^{\circ}$ & 11.67 & 12.50 & 12.28 & 12.15 & 12.67 & 14.00 & 13.11 & 13.26 & 10.67 & 11.00 & 11.44 & 11.04 \\
\hline D & 0 & $45^{\circ}$ & $90^{\circ}$ & 12.24 & 11.88 & 11.29 & 11.80 & 13.80 & 12.80 & 12.00 & 12.87 & 10.00 & 10.57 & 10.29 & 10.29 \\
\hline D & 1.5 & $45^{\circ}$ & $90^{\circ}$ & 29.94 & 29.24 & 31.06 & 30.08 & 36.91 & 36.36 & 39.55 & 37.61 & 17.17 & 16.17 & 15.50 & 16.28 \\
\hline D & 0 & $90^{\circ}$ & $45^{\circ}$ & 41.37 & 40.63 & 40.95 & 40.98 & 105.43 & 103.86 & 103.71 & 104.33 & 4.00 & 3.75 & 4.33 & 4.03 \\
\hline D & 1.5 & $90^{\circ}$ & $45^{\circ}$ & $\mid 69.67$ & 70.56 & \begin{tabular}{|l}
71.39 \\
\end{tabular} & 70.54 & 118.90 & 121.30 & 121.50 & 120.57 & 8.13 & 7.13 & 8.75 & 8.00 \\
\hline
\end{tabular}




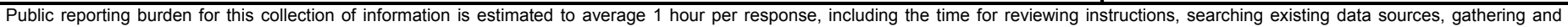

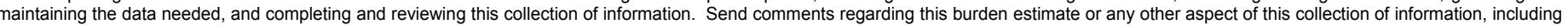

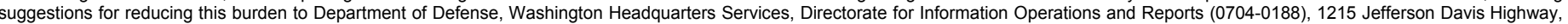

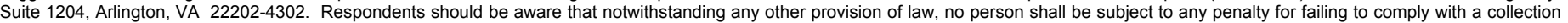
of information if it does not display a currently valid OMB control number. PLEASE DO NOT RETURN YOUR FORM TO THE ABOVE ADDRESS.
1. REPORT DATE (DD-MM-YYYY)
September 2006
4. TITLE AND SUBTITLE
2. REPORT TYPE
Final report

Expedient Gap Definition Using 3D LADAR

\begin{tabular}{l} 
3. DATES COVERED (FrOm - To) \\
5a. CONTRACT NUMBER \\
\hline 5b. GRANT NUMBER \\
5c. PROGRAM ELEMENT NUMBER \\
AT40-WP168A \\
5d. PROJECT NUMBER \\
P2115565 \\
\hline 5e. TASK NUMBER \\
\hline 5f. WORK UNIT NUMBER \\
8. PERFORMING ORGANIZATION \\
REPORT NUMBER
\end{tabular}

7. PERFORMING ORGANIZATION NAME(S) AND ADDRESS(ES) REPORT NUMBER

Geotechnical and Structures Laboratory

3909 Halls Ferry Road

ERDC/GSL TR-06-12

Vicksburg, MS 39180-6199

9. SPONSORING/MONITORING AGENCY NAME(S) AND ADDRESS(ES)

U.S. Army Corps of Engineers

Washington, DC 20314-1000

10. SPONSOR/MONITOR'S ACRONYM(S)

11. SPONSOR/MONITOR'S REPORT NUMBER(S)

12. DISTRIBUTION/AVAILABILITY STATEMENT

Approved for public release; distribution is unlimited.

\section{SUPPLEMENTARY NOTES}

\section{ABSTRACT}

This report presents a summary of the testing and analyses performed to determine the capabilities of a 3D LADAR (laser detection and ranging) system developed by Autonomous Solutions, Inc. (ASI). Under the direction of the U.S. Army Engineer Research and Development Center (ERDC), ASI has developed an algorithm to reduce the 3D point cloud acquired with the LADAR system into sets of 2D profiles that describe the terrain. This report provides data for (1) evaluating the ranging sensors that could potentially be used for measuring the geometry of a terrain gap, (2) reviewing results of the validation testing of the LADAR system, and (3) developing guidance for using LADAR systems to characterize terrain gaps. Validating the 3D LADAR system included the following tasks: (1) determining the effect of slope and depth of a gap on the measurements acquired with the LADAR system, (2) determining the effect of ambient light conditions on the measurements taken with the LADAR system,

(3) determining the effect of different surface materials/textures on the measurements taken with the LADAR system, and

(4) determining the effect of fog and dust on the measurements taken with the LADAR system. The project described in this report is part of the Battlespace Gap Definition and Defeat Army Technology Objective program.

\section{SUBJECT TERMS}

Gap definition

LADAR

Terrain mapping

\begin{tabular}{|c|c|c|c|c|c|}
\hline \multicolumn{3}{|c|}{ 16. SECURITY CLASSIFICATION OF: } & \multirow{2}{*}{$\begin{array}{l}\text { 17. LIMITATION } \\
\text { OF ABSTRACT }\end{array}$} & \multirow{2}{*}{$\begin{array}{l}\text { 18. NUMBER } \\
\text { OF PAGES } \\
108\end{array}$} & \multirow{2}{*}{\begin{tabular}{|l|} 
19a. NAME OF RESPONSIBLE PERSON \\
$\begin{array}{l}\text { 19b. TELEPHONE NUMBER (include area } \\
\text { code) }\end{array}$
\end{tabular}} \\
\hline $\begin{array}{l}\text { a. REPORT } \\
\text { UNCLASSIFIED }\end{array}$ & $\begin{array}{l}\text { b. ABSTRACT } \\
\text { UNCLASSIFIED }\end{array}$ & $\begin{array}{l}\text { c. THIS PAGE } \\
\text { UNCLASSIFIED }\end{array}$ & & & \\
\hline
\end{tabular}

